University of Tennessee Health Science Center

UTHSC Digital Commons

\title{
Repeated Zolpidem Treatment Effects on Sedative Tolerance, Withdrawal, mRNA Levels, and Protein Expression
}

\author{
Brittany T. Wright \\ University of Tennessee Health Science Center
}

Follow this and additional works at: https://dc.uthsc.edu/dissertations

Part of the Medicinal and Pharmaceutical Chemistry Commons, and the Neurosciences Commons

\section{Recommended Citation}

Wright, Brittany T. (http://orcid.org/0000-0002-1501-6596), "Repeated Zolpidem Treatment Effects on Sedative Tolerance, Withdrawal, mRNA Levels, and Protein Expression" (2016). Theses and Dissertations (ETD). Paper 406. http://dx.doi.org/10.21007/etd.cghs.2016.0408. 


\title{
Repeated Zolpidem Treatment Effects on Sedative Tolerance, Withdrawal, mRNA Levels, and Protein Expression
}

\begin{abstract}
Zolpidem and benzodiazepines (BZs) potentiate the inhibitory action of gamma-Aminobutyric acid (GABA) by allosterically binding to GABAA receptors (GABAAR). Prolonged use of GABAAR positive allosteric modulators (PAM) can lead to behavioral tolerance, the diminished response to the same drug dose with repeated use, and withdrawal, a group of symptoms that occur due to abrupt end of drug treatment. Zolpidem is a short-acting, non-BZ GABAAR PAM whose potential for tolerance and withdrawal is unclear. Zolpidem demonstrates sedative efficacy similar to BZs and has become a main treatment of insomnia in lieu of BZs. Zolpidem replaced BZs due to lower incidences of tolerance and withdrawal after prolonged treatment and discontinuation. Despite reported lower incidences, some studies find the occurrence of tolerance and withdrawal similar between zolpidem and BZs. Tolerance and withdrawal symptoms are likely caused by drug-induced neuroadaptive changes in central nervous system (CNS)

functioning, and these alterations may be similar between zolpidem and BZ. Past rodent research suggests that long term use of zolpidem and BZs may produce alterations in normal inhibitory GABAergic and excitatory glutamatergic functioning in the cortex, hippocampus, amygdala, and PFC and that these alterations may underlie sedative tolerance and withdrawal symptoms.

The purpose of this project was to examine the molecular mechanisms involved in the tolerance crosstolerance, and withdrawal of zolpidem and diazepam in C57/BL6J mice after different treatment durations. Elucidating the mechanisms behind zolpidem tolerance and withdrawal is necessary due to the ongoing usage of subunit specific GABAAR PAMs and, to a broader extent, an understanding of GABAARs themselves.

In Study 1, we measured sedative tolerance, cross-tolerance, and GABAAR associated mRNA levels in 4 regions of interest (ROI; the cortex, prefrontal cortex (PFC), hippocampus, and amygdala) after 3 days of intraperitoneal (i.p.) injections of diazepam and zolpidem in comparison to vehicle. We expected that this "short-term" exposure duration to diazepam and zolpidem would not result is tolerance, cross-tolerance, or changes in mRNA levels. Study 2 examined the same measures as in Study 1, in addition to AMPAR subunits, NDMAR subunits mRNA levels in the ROI, and total, surface, and intracellular GABAAR subunits protein expression due to 7 days of i.p. injections of diazepam and zolpidem compared to vehicle. Based on previous research both groups should become tolerant to zolpidem's sedative effects and show decreases of GABAAR subunits and increases in NMDAR subunits in the ROI. It is also expected that there will be decreases in total $\alpha 1$ and $\gamma 2$ in the cortex, a decrease of surface $\alpha 1$ in the cortex, and increases in GluR1 in the hippocampus after zolpidem and diazepam treatment. Study 3 measured the same measures as in Study 1 due to 30 days of i.p. injections of diazepam and zolpidem compared to vehicle. It was expected that both groups would become tolerant to zolpidem's sedative effects and show decreases of GABAAR subunits in the cortex, PFC, and hippocampus.
\end{abstract}

The development of sedative tolerance and cross-tolerance to the locomotor impairing effects (measure of sedation) of zolpidem was measured by activity in the open field. Spontaneous withdrawal was also measured by activity and anxiety like behavior in the open field. Flumazenil- induced withdrawal was measured by anxiety- like behaviors in the elevated plus maze (EPM), activity, and anxiety like behavior in the open field. Messenger RNA levels were measured by quantitative real time quantitative reverse transcription polymerase chain reaction (qRT-PCR) and protein expression was measured by western blot. The surface and intracellular proteins were separated using bissulfosuccinimidyl suberate (BS3) crosslinking. 
Three days of diazepam but not zolpidem resulted in cross-tolerance to zolpidem in Study 1 . Three days of zolpidem but not diazepam resulted in a decrease in the mRNA level of the $\alpha 5$ subunit in the hippocampus in Study 1. After 7 days of zolpidem or diazepam, mice were tolerant and cross-tolerant to zolpidem's sedative effects. Spontaneous withdrawal resulted in anxiety-like behavior and decreased locomotor activity. Flumazenil did induce a robust withdrawal syndrome as measured in the EPM or open field. Seven days of zolpidem and diazepam caused significant decreases in the mRNA expression of $\alpha 1$, $\alpha 3, \beta 2$, and $\delta$ GABAAR subunits in the cortex. Diazepam groups had significant decreases in the mRNA expression of $\alpha 4, \beta 1, \gamma 2$ subunits, GAT, and gephyrin in the cortex and significant decreases of $\alpha 5$ - and B3-GABAAR

subunits, and the GluN2A subunit in the hippocampus. Seven days of zolpidem resulted in a decrease in total $\alpha 2$ subunit protein level and 7 days of diazepam decreased total $\gamma 2$ subunit protein levels. Thirty days of diazepam but not zolpidem resulted in cross-tolerance to zolpidem in Study 3 . Thirty days of zolpidem but not diazepam resulted in a decrease in the mRNA levels of $\alpha 1, \alpha 2, \alpha 3, \beta 1, \beta 2, \beta 3, \gamma 1$, and $\gamma 2$ subunits in the PFC.

These results suggest that there is a window of time in which sedative tolerance to zolpidem is observed. The lack of zolpidem tolerance and minimal mRNA changes due to 3 days of zolpidem treatment may be due to its pharmacokinetic profile, zolpidem may not be in the system long enough to cause any changes. This may mean that sedative tolerance gradually develops and reaches detectable levels at later time points. Sedative tolerance and cross-tolerance to zolpidem is in line with other studies, however the spontaneous withdrawal is unique. Anxiety- like behavior and decreased activity were observed in our studies unlike other studies. The anxiety- like behavior is a common symptom of BZ withdrawal however the decrease in activity that was observed is not. It is unknown why this occurs though it may be due to a carryover sedative effect or more likely a placebo effect. Few studies have examined changes in protein levels. Study 2 found decreased protein expression of $\alpha 2$ and $\gamma 2$ in the cortex due to zolpidem and diazepam respectively, indicate GABAARs containing those subunits are associated with tolerance and cross-tolerance to zolpidem's sedative effects. This implies that the sedative effects of zolpidem is mediated by $\alpha 1-G A B A A R s$, the development of tolerance is mediated by $\alpha 2-G A B A A R s$ due to zolpidem binding to both. There was also a decrease in the intracellular $\alpha 1$ subunit which may indicate degradation of an intracellular pool of $\alpha 1$ subunits or $\alpha 1$-GABAARs. The lack of zolpidem tolerance due to 30 days of zolpidem may be due to increased metabolism displayed as an increase in CYP3A enzymes. It is unclear what effect the decrease in GABAAR subunits in the PFC due to 30 days of zolpidem implicate. These may affect tolerance to zolpidem's other effects such as amnesia.

\section{Document Type}

Dissertation

Degree Name

Doctor of Philosophy (PhD)

\section{Program}

Biomedical Sciences

\section{Research Advisor}

Scott A. Heldt, Ph.D.

\section{Keywords}

Zolpidem, Tolerance, Sedative, Withdrawal, Hypnotic, GABAA receptor 


\section{Subject Categories}

Medical Sciences | Medicinal and Pharmaceutical Chemistry | Medicine and Health Sciences |

Neurosciences | Pharmacy and Pharmaceutical Sciences 


\title{
Repeated Zolpidem Treatment Effects on Sedative Tolerance, Withdrawal, mRNA Levels, and Protein Expression
}

\author{
A Dissertation \\ Presented for \\ The Graduate Studies Council \\ The University of Tennessee \\ Health Science Center \\ In Partial Fulfillment \\ Of the Requirements for the Degree \\ Doctor of Philosophy \\ From The University of Tennessee
}

By

Brittany T. Wright

August 2016 
Portions of Chapters 2-5 (C) 2014 by Springer. All other material (C) 2016 by Brittany T. Wright. All rights reserved. 


\section{DEDICATION}

I would like to dedicate this dissertation to my family and friends who have supported me through this long journey. 


\section{ACKNOWLEDGEMENTS}

I would like to thank my mentor and committee chair Dr. Scott Heldt for his support and encouragement as well as my committee members, Drs. Kristin Hamre, Jeffery Steketee, Michael McDonald and Kazuko Sakata. I would like thank my lab mate Yudong Gao for his continued help throughout the years. I would also like to thank Kyle Summers for his continued support and love. 


\begin{abstract}
Zolpidem and benzodiazepines (BZs) potentiate the inhibitory action of gammaAminobutyric acid (GABA) by allosterically binding to $\mathrm{GABA}_{\mathrm{A}}$ receptors $\left(\mathrm{GABA}_{\mathrm{A}} \mathrm{R}\right)$. Prolonged use of $\mathrm{GABA}_{\mathrm{A}} \mathrm{R}$ positive allosteric modulators (PAM) can lead to behavioral tolerance, the diminished response to the same drug dose with repeated use, and withdrawal, a group of symptoms that occur due to abrupt end of drug treatment. Zolpidem is a short-acting, non-BZ GABA $\mathrm{A}_{\mathrm{R}}$ PAM whose potential for tolerance and withdrawal is unclear. Zolpidem demonstrates sedative efficacy similar to BZs and has become a main treatment of insomnia in lieu of BZs. Zolpidem replaced BZs due to lower incidences of tolerance and withdrawal after prolonged treatment and discontinuation. Despite reported lower incidences, some studies find the occurrence of tolerance and withdrawal similar between zolpidem and BZs. Tolerance and withdrawal symptoms are likely caused by drug-induced neuroadaptive changes in central nervous system (CNS) functioning, and these alterations may be similar between zolpidem and BZ. Past rodent research suggests that long term use of zolpidem and BZs may produce alterations in normal inhibitory GABAergic and excitatory glutamatergic functioning in the cortex, hippocampus, amygdala, and PFC and that these alterations may underlie sedative tolerance and withdrawal symptoms.
\end{abstract}

The purpose of this project was to examine the molecular mechanisms involved in the tolerance cross-tolerance, and withdrawal of zolpidem and diazepam in C57/BL6J mice after different treatment durations. Elucidating the mechanisms behind zolpidem tolerance and withdrawal is necessary due to the ongoing usage of subunit specific $\mathrm{GABA}_{\mathrm{A}} \mathrm{R}$ PAMs and, to a broader extent, an understanding of GABA $\mathrm{A}_{\mathrm{A}}$ themselves.

In Study 1, we measured sedative tolerance, cross-tolerance, and $\mathrm{GABA}_{\mathrm{A}} \mathrm{R}$ associated mRNA levels in 4 regions of interest (ROI; the cortex, prefrontal cortex (PFC), hippocampus, and amygdala) after 3 days of intraperitoneal (i.p.) injections of diazepam and zolpidem in comparison to vehicle. We expected that this "short-term" exposure duration to diazepam and zolpidem would not result is tolerance, crosstolerance, or changes in mRNA levels. Study 2 examined the same measures as in Study 1, in addition to AMPAR subunits, NDMAR subunits mRNA levels in the ROI, and total, surface, and intracellular $\mathrm{GABA}_{\mathrm{A}} \mathrm{R}$ subunits protein expression due to 7 days of i.p. injections of diazepam and zolpidem compared to vehicle. Based on previous research both groups should become tolerant to zolpidem's sedative effects and show decreases of $\mathrm{GABA}_{\mathrm{A}} \mathrm{R}$ subunits and increases in NMDAR subunits in the ROI. It is also expected that there will be decreases in total $\alpha 1$ and $\gamma 2$ in the cortex, a decrease of surface $\alpha 1$ in the cortex, and increases in GluR1 in the hippocampus after zolpidem and diazepam treatment. Study 3 measured the same measures as in Study 1 due to 30 days of i.p. injections of diazepam and zolpidem compared to vehicle. It was expected that both groups would become tolerant to zolpidem's sedative effects and show decreases of $\mathrm{GABA}_{\mathrm{A}} \mathrm{R}$ subunits in the cortex, PFC, and hippocampus. 
The development of sedative tolerance and cross-tolerance to the locomotor impairing effects (measure of sedation) of zolpidem was measured by activity in the open field. Spontaneous withdrawal was also measured by activity and anxiety like behavior in the open field. Flumazenil-induced withdrawal was measured by anxiety-like behaviors in the elevated plus maze (EPM), activity, and anxiety like behavior in the open field. Messenger RNA levels were measured by quantitative real time quantitative reverse transcription polymerase chain reaction (qRT-PCR) and protein expression was measured by western blot. The surface and intracellular proteins were separated using bissulfosuccinimidyl suberate (BS3) cross-linking.

Three days of diazepam but not zolpidem resulted in cross-tolerance to zolpidem in Study 1. Three days of zolpidem but not diazepam resulted in a decrease in the mRNA level of the 25 subunit in the hippocampus in Study 1. After 7 days of zolpidem or diazepam, mice were tolerant and cross-tolerant to zolpidem's sedative effects. Spontaneous withdrawal resulted in anxiety-like behavior and decreased locomotor activity. Flumazenil did induce a robust withdrawal syndrome as measured in the EPM or open field. Seven days of zolpidem and diazepam caused significant decreases in the mRNA expression of $\alpha 1, \alpha 3, \beta 2$, and $\delta \mathrm{GABA}_{\mathrm{A}} \mathrm{R}$ subunits in the cortex. Diazepam groups had significant decreases in the mRNA expression of $\alpha 4, \beta 1, \gamma 2$ subunits, GAT, and gephyrin in the cortex and significant decreases of $\alpha 5$ - and $\beta 3-\mathrm{GABA}_{\mathrm{A}} \mathrm{R}$ subunits, and the GluN2A subunit in the hippocampus. Seven days of zolpidem resulted in a decrease in total $\alpha 2$ subunit protein level and 7 days of diazepam decreased total $\gamma 2$ subunit protein levels. Thirty days of diazepam but not zolpidem resulted in crosstolerance to zolpidem in Study 3. Thirty days of zolpidem but not diazepam resulted in a decrease in the mRNA levels of $\alpha 1, \alpha 2, \alpha 3, \beta 1, \beta 2, \beta 3, \gamma 1$, and $\gamma 2$ subunits in the PFC.

These results suggest that there is a window of time in which sedative tolerance to zolpidem is observed. The lack of zolpidem tolerance and minimal mRNA changes due to 3 days of zolpidem treatment may be due to its pharmacokinetic profile, zolpidem may not be in the system long enough to cause any changes. This may mean that sedative tolerance gradually develops and reaches detectable levels at later time points. Sedative tolerance and cross-tolerance to zolpidem is in line with other studies, however the spontaneous withdrawal is unique. Anxiety- like behavior and decreased activity were observed in our studies unlike other studies. The anxiety-like behavior is a common symptom of BZ withdrawal however the decrease in activity that was observed is not. It is unknown why this occurs though it may be due to a carryover sedative effect or more likely a placebo effect. Few studies have examined changes in protein levels. Study 2 found decreased protein expression of $\alpha 2$ and $\gamma 2$ in the cortex due to zolpidem and diazepam respectively, indicate $\mathrm{GABA}_{\mathrm{A}} \mathrm{Rs}_{\mathrm{s}}$ containing those subunits are associated with tolerance and cross-tolerance to zolpidem's sedative effects. This implies that the sedative effects of zolpidem is mediated by $\alpha 1-\mathrm{GABA}_{\mathrm{A}} \mathrm{Rs}$, the development of tolerance is mediated by $\alpha 2-\mathrm{GABA}_{\mathrm{A}} \mathrm{Rs}$ due to zolpidem binding to both. There was also a decrease in the intracellular $\alpha 1$ subunit which may indicate degradation of an intracellular pool of $\alpha 1$ subunits or $\alpha 1-\mathrm{GABA}_{\mathrm{A}}$ Rs. The lack of zolpidem tolerance due to 30 days of zolpidem may be due to increased metabolism displayed as an increase in CYP3A enzymes. It is unclear what effect the decrease in $\mathrm{GABA}_{\mathrm{A}} \mathrm{R}$ subunits in the PFC due to 30 days of 
zolpidem implicate. These may affect tolerance to zolpidem's other effects such as amnesia. 


\section{TABLE OF CONTENTS}

CHAPTER 1. INSOMNIA TREATMENT ISSUES....................................................1

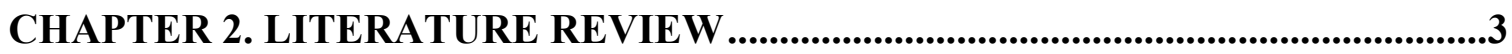

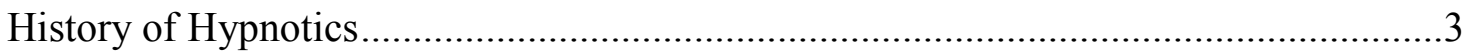

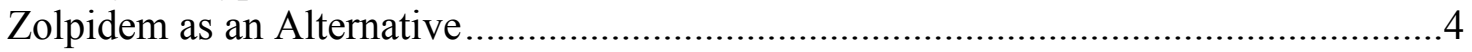

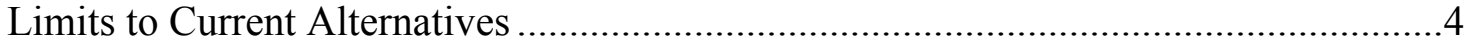

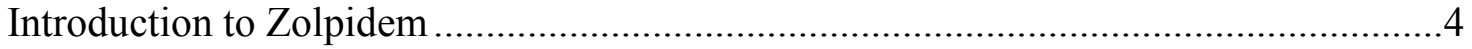

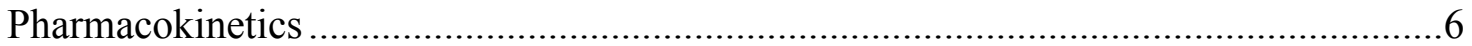

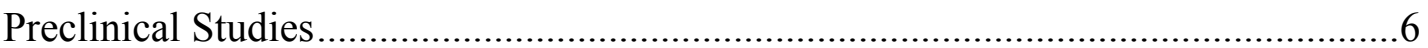

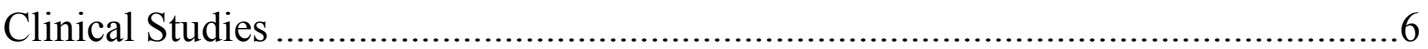

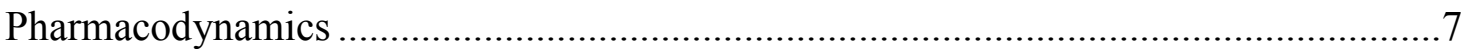

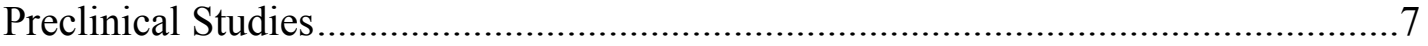

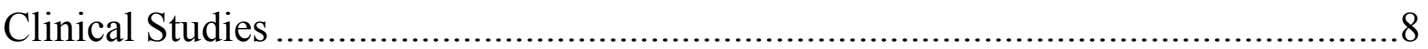

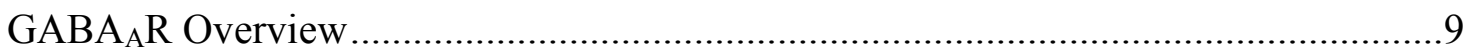

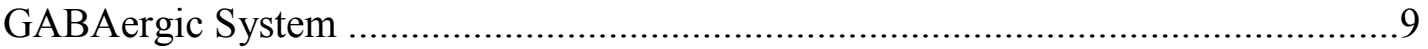

$\mathrm{GABA}_{\mathrm{A}} \mathrm{R}$ Subunit Localization and Distribution .......................................................

$\mathrm{GABA}_{\mathrm{A}} \mathrm{R}$ Subtypes and Modulatory Effects .........................................................13

Contributions of GABA and Glutamate mRNA and Protein Alterations in the

Cortex, PFC, Hippocampus, and Amygdala to Sedative Tolerance and

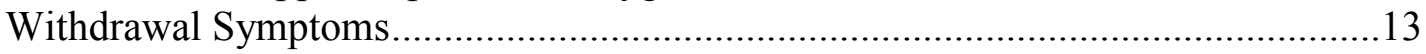

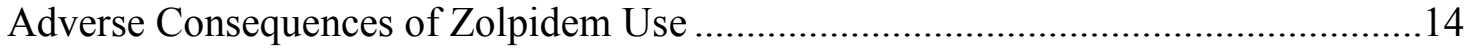

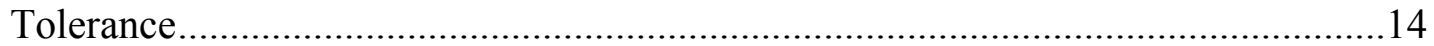

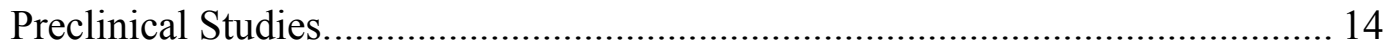

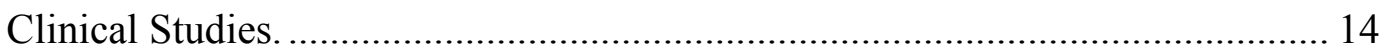

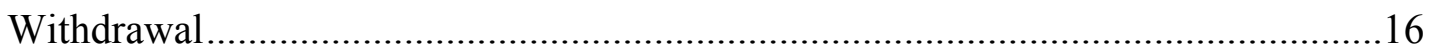

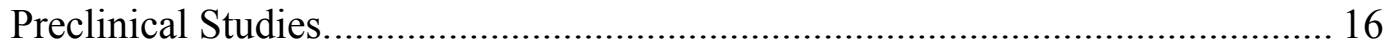

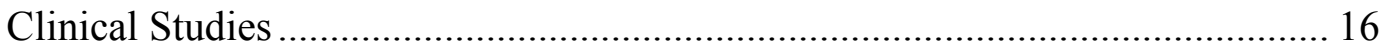

Relationship Between Duration of Zolpidem Use and the Emergence of

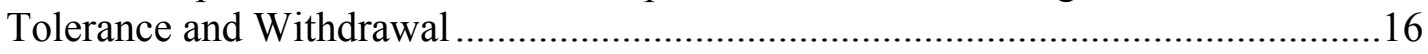

Usefulness of Rodent Models of Sedation and Limitations.......................................17

Abuse, Dependence, and Addiction ..................................................................18

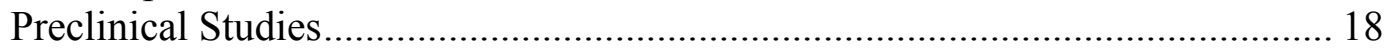

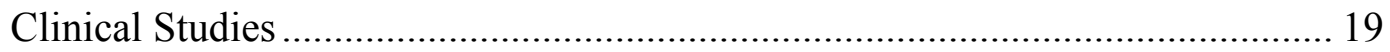

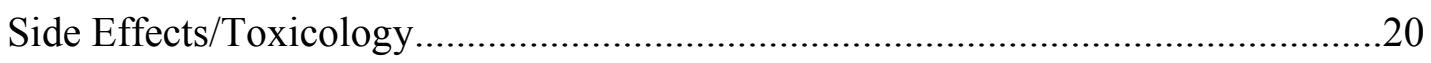

Neuropharmacology and Neurocircuitry of Adverse Effects ......................................20

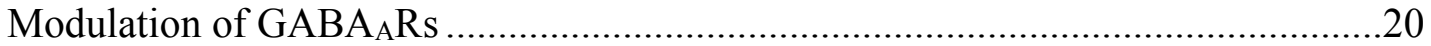

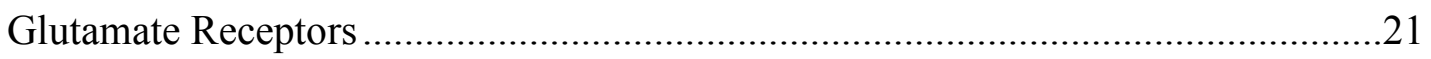

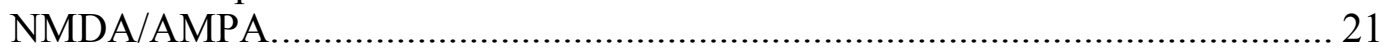

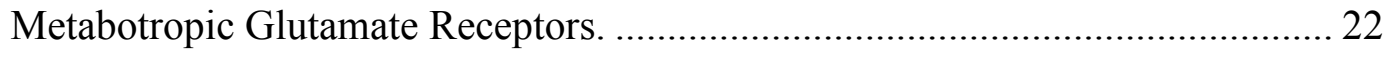

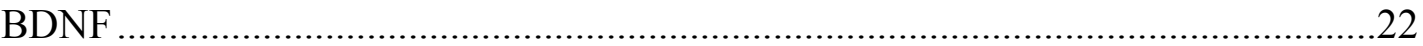

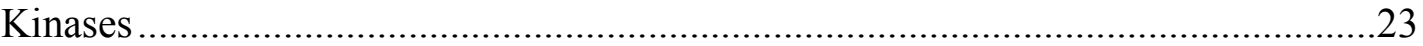

Influence of GABA Transmission on Sleep, Sedation, Motor Inhibition, and

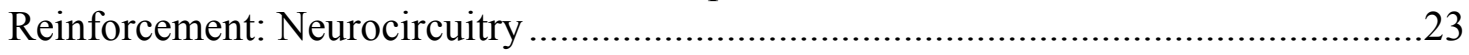




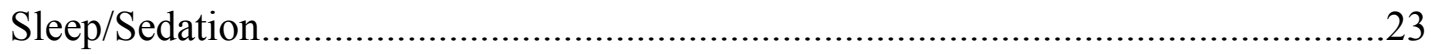

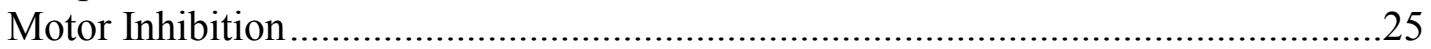

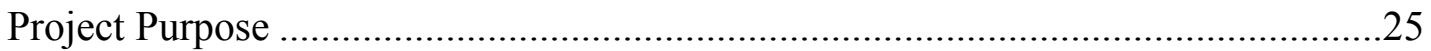

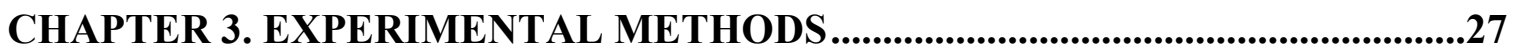

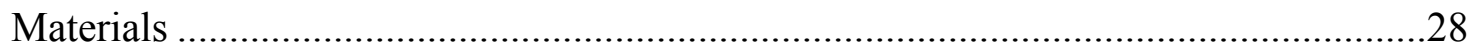

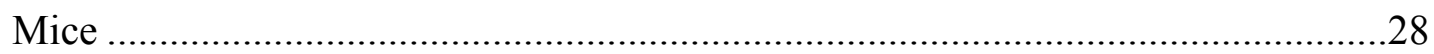

Drugs and Antibodies .................................................................................28

Justification for Why We Selected 3, 7, and 30 Days as Our Experimental Time

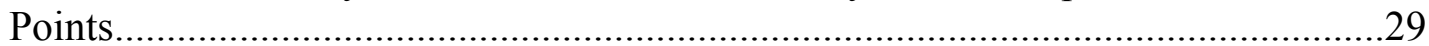

Justification for Two Injections for Methodology ................................................29

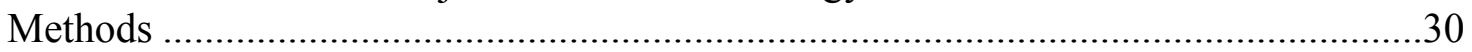

Tolerance and Spontaneous Withdrawal: Open Field.................................................30

Flumazenil-Induced Withdrawal: EPM and Open Field ...........................................32

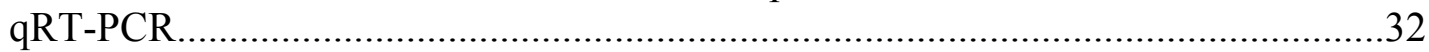

Bissulfosuccinimidyl Suberate (BS3) Cross-Linking/Western Blot.............................35

Data Reduction and Statistical Analyses ..................................................................36

Tolerance, Spontaneous, and Flumazenil-Induced Withdrawal. ........................... 36

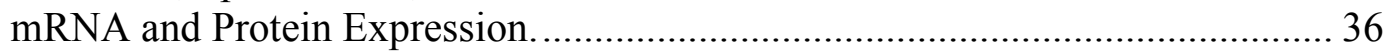

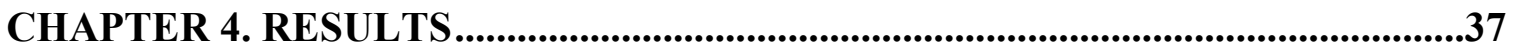

Study 1: The Effect of 3-Day Zolpidem and Diazepam Treatment on Behavioral

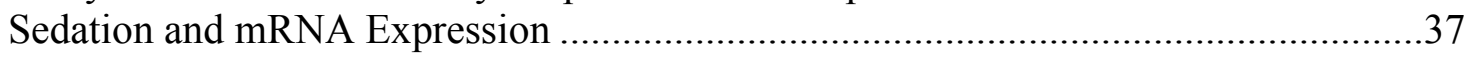

Tolerance to the Sedative Effects of Zolpidem..........................................................37

Changes in mRNA Expression Measured by qRT-PCR………………………….....37

Study 2: The Effect of 7-Day Zolpidem and Diazepam Treatment on Behavioral

Sedation, Withdrawal, mRNA, and Protein Expression................................................41

Tolerance to the Sedative Effects of Zolpidem..........................................................

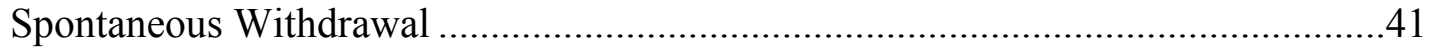

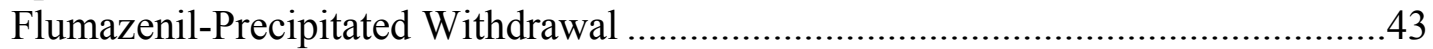

Changes in mRNA Expression Measured by qRT-PCR.............................................48

Changes in Total and Surface Subunit Protein Expression ........................................54

Study 3: The Effect of 30-Day Zolpidem and Diazepam Treatment on Behavioral

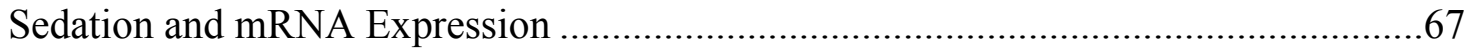

Tolerance to the Sedative Effects of Zolpidem........................................................67

Changes in mRNA Expression Measured by qRT-PCR.............................................69

Comparisons of Zolpidem and Diazepam Sedative Effects after 3-, 7-, and 30-Day

Treatment Durations ………………………………...........................................69

Comparison of Center, and Periphery Distance Traveled: Spontaneous Withdrawal...73

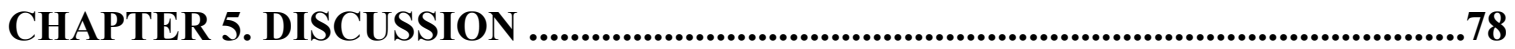

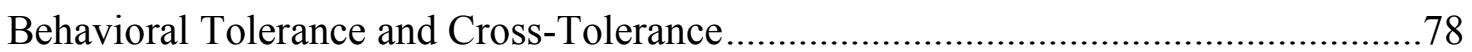

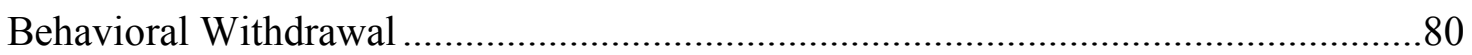

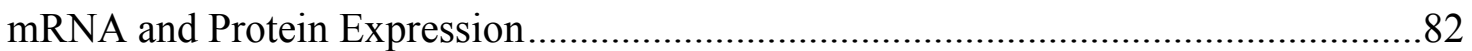

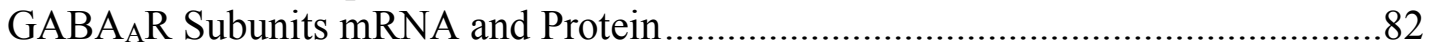

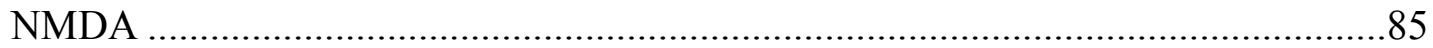


Connection Between Sedation, Withdrawal, and Observed Alterations in the mRNA and Protein Levels in the Cortex, PFC, Hippocampus, and Amygdala ........86

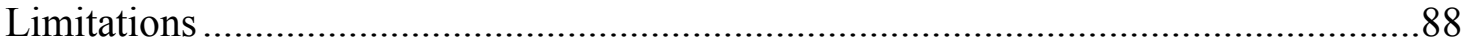

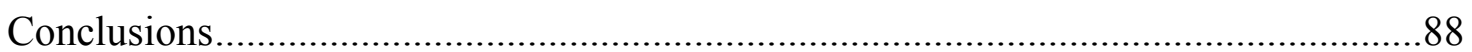

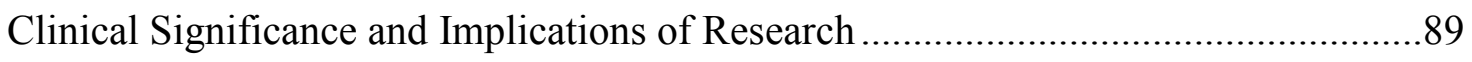

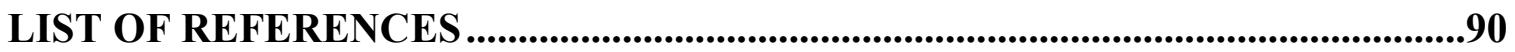

APPENDIX. MRNA EXPRESSION AFTER 1 DAY OF VEH, DZP, AND

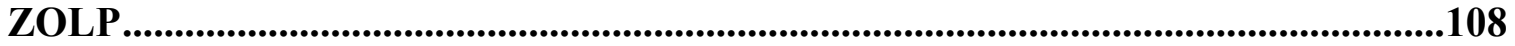

VITA 


\section{LIST OF TABLES}

Table 2-1. Case reports on dependence/abuse of zolpidem .......................................15

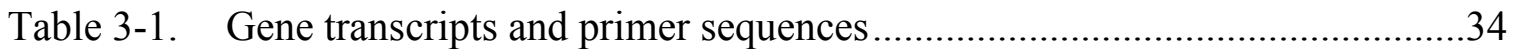




\section{LIST OF FIGURES}

Figure 2-1. Comparison of zolpidem and diazepam structures ........................................5

Figure 2-2. Example of a typical GABA $\mathrm{A}_{\mathrm{A}}$ complex.................................................10

Figure 2-3. Example of the localization of synaptic and extrasynaptic $\mathrm{GABA}_{A} \mathrm{R}$ opposite the presynaptic cleft

Figure 2-4. Example of differential regional distribution of $\alpha$ subunits proteins in rat brain sensitive to BZs

Figure 2-5. $\mathrm{GABA}_{\mathrm{A}} \mathrm{Rs}$ modulated in the arousal system

Figure 3-1. Overview flow chart of experimental studies

Figure 4-1. Decreased locomotor impairing effect of zolpidem after 3-day administration of zolpidem, diazepam, or vehicle

Figure 4-2. Relative levels of mRNA in the cortex, amygdala, and pfe following 3day administration of zolpidem, diazepam, or vehicle

Figure 4-3. Relative levels of mRNA in the hippocampus following 3-day administration of zolpidem, diazepam, or vehicle

Figure 4-4. Decreased locomotor impairing effect of zolpidem after 7-day administration of zolpidem, diazepam, or vehicle

Figure 4-5. Decreased locomotor activity in zolpidem and diazepam groups after cessation of 7 days of zolpidem and diazepam

Figure 4-6. Decreased percent time in center, center entries, and rearing frequency after cessation of 7 days of zolpidem and diazepam

Figure 4-7. Percent time spent and percent entries into the open arms of the EPM after acute flumazenil administration after 7-day administration of zolpidem, diazepam, or vehicle.....

Figure 4-8. Locomotor effect of flumazenil on test day 1 after 7-days administration of zolpidem, diazepam, or vehicle

Figure 4-9. Effect of flumazenil on percent time in center, center entries, and rearing frequency on test day 1 after 7-days administration of zolpidem, diazepam, or vehicle

Figure 4-10. Locomotor effect of flumazenil test day 2 after 7-day administration of zolpidem, diazepam, or vehicle 
Figure 4-11. Effect of flumazenil on percent time in center, center entries, and rearing frequency on test day 2 after 7-days administration of zolpidem, diazepam, or vehicle

Figure 4-12. Relative levels of mRNA in the cortex following 7-day administration of zolpidem, diazepam, or vehicle

Figure 4-13. Relative levels of mRNA in the hippocampus following 7-day administration of zolpidem, diazepam, or vehicle

Figure 4-14. Relative levels of mRNA in the amygdala and pfc following 7-day administration of zolpidem, diazepam, or vehicle

Figure 4-15. Relative levels of mRNA in the hippocampus following 7-day administration of zolpidem, diazepam, or vehicle

Figure 4-16. Relative levels of mRNA in the cortex, amygdala, and pfc following 7day administration of zolpidem, diazepam, or vehicle

Figure 4-17. Relative $\alpha 2$ subunit expression in the cortex following 7-day administration of zolpidem, diazepam, or vehicle

Figure 4-18. Relative $\gamma 2$ subunit expression in the cortex following 7-day administration of zolpidem, diazepam, or vehicle

Figure 4-19. Relative $\alpha 1$ subunit expression in the cortex following 7-day administration of zolpidem, diazepam, or vehicle

Figure 4-20. Relative $\alpha 3$ subunit expression in the cortex following 7-day administration of zolpidem, diazepam, or vehicle

Figure 4-21. Relative GluN1 subunit expression in the cortex following 7-day administration of zolpidem, diazepam, or vehicle

Figure 4-22. Relative GluN2A subunit expression in the cortex following 7-day administration of zolpidem, diazepam, or vehicle

Figure 4-23. BS3 treated samples, surface and intracellular $\alpha 1$ protein expression levels in the cortex following 7-day administration of zolpidem, diazepam, or vehicle

Figure 4-24. Relative $\alpha 1$ protein expression levels in the hippocampus following 7day administration of zolpidem, diazepam, or vehicle

Figure 4-25. Relative $\alpha 2$ protein expression levels in the hippocampus following 7day administration of zolpidem, diazepam, or vehicle..... 
Figure 4-26. Decreased locomotor impairing effect of zolpidem after 30-day administration of zolpidem, diazepam, or vehicle .68

Figure 4-27. Relative levels of mRNA in the pfc following 30-day administration of zolpidem, diazepam, or vehicle.

Figure 4-28. Relative levels of mRNA in the cortex, hippocampus, and amygdala following 7-day administration of zolpidem, diazepam, or vehicle.

Figure 4-29. No significant differences between vehicle groups from each study on motor activity

Figure 4-30. No significant differences between diazepam groups from each study on motor activity.

Figure 4-31. Increased motor activity due to 7 days of ZOLP compared to 3 or 30 days of ZOLP .

Figure 4-32. Decreased motor activity in the center region of the open field apparatus...76

Figure 4-33. No significant differences between groups in the peripheral region of the open field apparatus on motor activity 


\section{LIST OF ABBREVIATIONS}

\begin{tabular}{|c|c|}
\hline $\begin{array}{l}\text { ACSF } \\
\alpha \mathrm{x}-\mathrm{GABA}_{\mathrm{A}} \mathrm{Rs}\end{array}$ & $\begin{array}{l}\text { Artificial cerebrospinal fluid } \\
\alpha \mathrm{x} \text {-containing } \mathrm{GABA}_{\mathrm{A}} \mathrm{Rs}\end{array}$ \\
\hline AMPA & $\alpha$-amino-3-hydroxy-5-methyl-4-isoxazolepropionic acid \\
\hline AUC & Area under the curve \\
\hline BDNF & Brain-derived neurotrophic factor \\
\hline BS3 & Bissulfosuccinimidyl suberate \\
\hline BLA & Basolateral amygdala \\
\hline BZ & Benzodiazepine \\
\hline CDP & Chlordiazepoxide \\
\hline CeA & Central amygdala \\
\hline cDNA & Complementary DNA \\
\hline Cmax & Maximum serum concentration \\
\hline CNS & Central nervous system \\
\hline CPP & Conditioned place preference \\
\hline $\mathrm{Ct}$ & Threshold value \\
\hline CYP450 & Cytochrome P450 \\
\hline DEA & Drug Enforcement Administration \\
\hline DSM-V & Diagnostic and Statistical Manual of Mental Disorders, $5^{\text {th }}$ Edition \\
\hline ED50 & Median effective dose \\
\hline EMG & Electromyography \\
\hline EPM & Elevated plus maze. \\
\hline FDA & Food and Drug Administration \\
\hline GABA & Gamma aminobutyric acid \\
\hline $\mathrm{GABA}_{\mathrm{A}} \mathrm{R}$ & Gamma aminobutyric A Receptors \\
\hline GABARAP & GABA receptor-associated protein \\
\hline GAT-1 & GABA transporter 1 \\
\hline GluN2A & NMDAR 2A \\
\hline ICER & Inducible cAMP early repressor \\
\hline ICSS & Intracranial self-stimulation \\
\hline I.P. & Intraperitoneal \\
\hline I.V. & Intravenously \\
\hline $\mathrm{KCC} 2$ & $\mathrm{~K}-\mathrm{Cl}$ cotransporter 2 \\
\hline $\mathrm{KO}$ & Knockout mouse \\
\hline LC & Locus caeruleus \\
\hline LORR & Loss of righting reflex \\
\hline $\mathrm{MeCP} 2$ & Methyl-CPG binding protein 2 \\
\hline mIPSC & Miniature inhibitory post-synaptic currents \\
\hline mGluR & Metabotropic glutamate receptors \\
\hline $\mathrm{MnPO}$ & Median preoptic nucleus \\
\hline nAChR & Nicotinic acetylcholine receptors \\
\hline $\mathrm{NKCC} 2$ & $\mathrm{Na}-\mathrm{K}-\mathrm{Cl}$ cotransporter 2 \\
\hline NMDA & N-methyl-D-aspartate \\
\hline NMDAR & N-methyl-D-aspartate receptor \\
\hline
\end{tabular}




$\begin{array}{ll}\text { NREM } & \text { Non-rapid eye movement } \\ \text { PAS } & \text { Precipitated withdrawal score } \\ \text { PFC } & \text { Prefrontal Cortex } \\ \text { PM-DAT } & \text { Plus maze discriminative avoidance task } \\ \text { PPN } & \text { Pedunculopontine nucleus } \\ \text { PTZ } & \text { Pentylenetetrazol } \\ \text { qRT-PCR } & \text { Quantitative real-time polymerase chain reaction } \\ \text { SICI } & \text { Short interval intracortical inhibition } \\ \text { SNr } & \text { Substantia nigra pars reticulate } \\ \text { SWS } & \text { Slow wave sleep } \\ \mathrm{T}_{1 / 2} & \text { Half-life } \\ \mathrm{T}_{\max } & \text { Time of peak concentration } \\ \mathrm{V}_{\mathrm{d}} & \text { Volume of distribution } \\ \text { vLPO } & \text { Ventrolateral preoptic area }\end{array}$




\section{CHAPTER 1. INSOMNIA TREATMENT ISSUES}

Clinically, the sleep disorder insomnia is defined as difficulty initiating or maintaining sleep [1]. Treatment of insomnia is of particular importance because the estimated prevalence within the United States (U.S.) adult population is $10 \%$ to $30 \%$. Insomnia is also often comorbid with the psychiatric disorders anxiety and depression [2, 3]. Until recently, traditional benzodiazepines (BZs) diazepam, alprazolam, and clonazepam were the first-line clinical medications for insomnia. However, the unwanted side effects of daytime drowsiness, dizziness, confusion and nausea often resulted from BZ use. In addition to unwanted side-effects, long term use of BZs could cause tolerance (defined as the need to markedly increase the amount of the substance to achieve the desired effect) and withdrawal symptoms (anxiety, insomnia, and seizures that develop after cessation of long term BZ treatment) $[4,5]$. Tolerance and withdrawal are commonly associated with dependence (a desire often strong, sometimes overpowering to take psychoactive drugs) and addiction (a compulsive drive to take rewarding stimuli regardless of risk) $[1,4,6-8]$. Dependence and withdrawal are serious issues that can impede the treatment of insomnia and research into safer alternatives has been ongoing.

Over the past decade, the imidazopyridine zolpidem (brand name Ambien) has replaced BZs as the most commonly prescribed drug for patients suffering from insomnia in the U.S. Both zolpidem and BZs enhance the actions of the inhibitory neurotransmitter gamma-aminobutyric acid (GABA) at $\mathrm{GABA}_{\mathrm{A}}$ receptors $\left(\mathrm{GABA}_{\mathrm{A}} \mathrm{Rs}\right)$. BZs exhibit nonpreferential binding to $\alpha 1-, \alpha 2-, \alpha 3$, and $\alpha 5$-containing GABA ${ }_{A} \operatorname{Rs}(\alpha 1-, \alpha 2-, \alpha 3-$, and $\alpha 5-$ $\left.\mathrm{GABA}_{\mathrm{A}} \mathrm{Rs}\right)$. Zolpidem, however, exhibits preferential binding and efficacy at $\alpha 1$ $\mathrm{GABA}_{\mathrm{A}} \mathrm{Rs}$ alone and produces therapeutic sedative effects. Zolpidem is purportedly safer and has fewer side effects than traditionally used BZs when used properly. However, recent studies have shown that zolpidem may be more similar to BZs in terms of behavioral tolerance, withdrawal, abuse potential, and physical dependence liability. In fact, the frequency of zolpidem abuse and dependence is similar to that of BZs within the population that has a prior history of $\mathrm{BZ}$ and alcohol abuse.

There has been conflicting evidence concerning zolpidem's ability to induce behavioral tolerance and withdrawal in animal models after various treatment doses and durations. Also unclear is the degree to which repeated administration of zolpidem can produce neuroadaptive changes in GABAergic and glutamatergic function that may underlie tolerance and withdrawal. Initial rodent studies indicated little evidence of sedative tolerance to zolpidem, however more recent studies present evidence that tolerance can develop to zolpidem's sedative effects. It is known that diazepam tolerance and withdrawal can been detected as early as 1 day and as late as 30 days. It is hypothesized that BZ tolerance and withdrawal may be caused by overlapping neuroadaptive changes. The purpose of this project is to examine tolerance, crosstolerance, withdrawal, and molecular alterations in zolpidem and diazepam-treated C57/BL6J mice. Elucidating evidence of tolerance, withdrawal, and neuroadaptive changes after repeated zolpidem use is necessary due to the ongoing usage of subunit specific $\mathrm{GABA}_{\mathrm{A}} \mathrm{R}$ positive allosteric modulators (PAMs) like zolpidem and, to a broader 
extent, an understanding of $\mathrm{GABA}_{\mathrm{A}} \mathrm{Rs}$. Furthermore, given the use of genetically engineered animal models for understanding $\mathrm{GABA}_{\mathrm{A}} \mathrm{R}$ function, knowledge about the mechanisms underlying tolerance in common background strains will be helpful in interpreting knockout and transgenic mouse studies that attempt to identify genetic factors that can play a role in drug abuse susceptibility. 


\section{CHAPTER 2. LITERATURE REVIEW*}

\section{History of Hypnotics}

The earliest known sedative-hypnotics (drugs used to induce or maintain sleep) were alcohol and opium. In the 17th century, the combination of opium and alcohol was packaged by the name laudanum for the treatment of insomnia and other medical conditions. Laudanum was discontinued due to high incidences of dependence, addiction, and death [9]. Other drugs would be developed to treat insomnia. Of those treatments barbiturates were of prominent use. Barbiturates were developed in the late 1800s and used throughout the 1950s. The first barbiturates were synthesized in 1864 by Adolph Von Baeyer and refined by Edouard Grimaux [10]. The first barbiturate approved for clinical use was diethyl-barbituric acid, known by barbital. Barbital was synthesized in 1881 by Max Conrad and M. Guthzeit and marketed to consumers as a sedative-hypnotic in 1904 [10]. After barbital, over 2500 agents based on barbital were created, including phenobarbital, occasionally used to treat insomnia to this day. The use of barbiturates as sedative-hypnotics peaked in the 1930s and 1940s, but usage declined due to high occurrences of addiction and death by overdose.

BZs replaced barbiturates as hypnotics because of their lower incidences of addiction and overdose compared to barbiturates. The safer alternatives to barbiturates, BZs were created by Dr. Leo Sternbach in the 1930s'. The first BZ, Ro 5-0690 or chlordiazepoxide (CDP), had hypnotic, sedative, and anticonvulsant effects. CDP was marketed for anxiety in 1960 followed by diazepam, marketed for multiple uses including the treatment for insomnia in 1963. As more BZs were developed, research into BZ function found that $B Z s$ are positive modulators of $G_{A B A}$ receptors $\left(G A B A_{A} R s\right)$ like barbiturates and bind to $\omega 1$ ( $\alpha 1$-containing) and $\omega 2\left(\alpha 2-, \alpha 3-, \alpha 5\right.$-containing) GABA $\mathrm{A}_{\mathrm{AS}}$ $[11,12]$. It was thought that preferential binding to this site made BZs safer and resulted in the absence of the abuse and addiction patients developed with barbiturates.

Unfortunately BZs displayed the same propensity for dependence and addiction as barbiturates [5, 13, 14]. Long term BZ use in humans, defined as greater than one year, could induce tolerance and withdrawal symptoms. The development of tolerance and withdrawal symptoms are more likely to occur in patients with a history of previous BZ, opioid, or ethanol abuse and patients with anxiety or depression [15]. The development of safer alternatives that have low to no incidences of tolerance and withdrawal effects have been ongoing and have led to the recent development of sedative-hypnotic non-BZs that should have a reduced capacity for tolerance.

\footnotetext{
* Modified with permission. Wright, B.T., C.F. Gluszek, and S.A. Heldt, The effects of repeated zolpidem treatment on tolerance, withdrawal-like symptoms, and GABAA receptor $m R N A$ s profile expression in mice: comparison with diazepam. Psychopharmacology (Berl), 2014. 231(15): p. 2967-79 [16].
} 


\section{Zolpidem as an Alternative}

The ongoing issue of dependence to sedative-hypnotics (e.g. barbiturate and BZs) has motivated research into safer alternatives. These alternatives include zolpidem, zopiclone, zaleplon, and eszopiclone, non-BZs of distinct structural, pharmacokinetic and pharmacodynamic properties. Zolpidem was synthesized in 1978 by Jean-Pierre Kaplan and George Pascal at Synthélabo Laboratories and patented in the U.S. in 1983. The pharmaceutical company Sanofi marketed zolpidem as a sleep aid in the European market in 1988 and the U.S. market in 1992. Compared to BZs, zolpidem is thought to be a superior sedative-hypnotic due to its preferential binding and efficacy of $\mathrm{GABA}_{\mathrm{A}} \mathrm{Rs}$ containing the $\alpha 1$ subunit $\left(\alpha 1-\mathrm{GABA}_{\mathrm{A}} \mathrm{R}\right)$ and lower affinity to GABA $\mathrm{A}_{\mathrm{A}}$ s containing $\alpha 2$, $\alpha 3$, and $\alpha 5$ subunits. In contrast, BZs have similar binding and efficacy at $\alpha 1-, \alpha 2-, \alpha 3-$, and $\alpha 5-\mathrm{GABA}_{\mathrm{A}} \mathrm{Rs}[17,18]$. Zolpidem's unique pharmacology results in fast acting sedative-hypnotic effects that are therapeutically advantageous for inducing sleep [4]. Past research has revealed that positive modulation of $\alpha 1-\mathrm{GABA}_{\mathrm{A}} \mathrm{Rs}$ induces sedation, sleep, amnesia, and anticonvulsant effects $[18,19]$. Zolpidem is currently one of the most widely used sedative-hypnotics in the U.S., and under the brand name Ambien $\mathbb{C}$, has become a popular sedative-hypnotic with yearly sales of more than 2 billion dollars in the U.S. $[20,21]$. However some studies suggest the propensity for tolerance and withdrawal symptoms to zolpidem is similar to BZs $[22,23]$.

\section{Limits to Current Alternatives}

The preferential affinity and binding of zolpidem to $\alpha 1-\mathrm{GABA}_{\mathrm{A}} \mathrm{Rs}$ was believed to decrease the development of tolerance and withdrawal symptoms produced by long term use. To date, there have been conflicting evidence of development of tolerance and withdrawal to zolpidem- in both human and rodent studies [24-27]. Early evidence in rodents suggested that chronic zolpidem administration at relatively high dose levels (e.g. $60 \mathrm{mg} / \mathrm{kg}$ ) does not induce tolerance [24]. However, recent studies suggest lower dose levels of zolpidem (e.g. 1.25-10 mg/kg) in rodents can induce tolerance [24, 28, 29]. Compared to humans, clinical studies do not find evidence of tolerance and withdrawal at clinical dose levels (e.g. $5-10 \mathrm{mg} / \mathrm{kg}$ ), but case studies do find evidence of tolerance and withdrawal in humans at large dose levels (e.g.200 mg/kg) of zolpidem [27, 30]. Thus, numerous studies have focused on how zolpidem, BZs, other GABA ${ }_{A} R$ modulators affect GABAergic transmission might lead to tolerance and withdrawal.

\section{Introduction to Zolpidem}

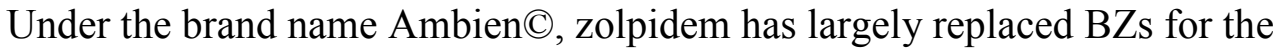
treatment of insomnia [20]. The chemical name for zolpidem is N, N, 6-trimethyl-2-(4methylphenyl) imidazo [1,2-a] pyridine-3-acetamide hemitartrate and is an imidazopyridine, structurally distinct from BZs (Figure 2-1) [31, 32]. Zolpidem is widely

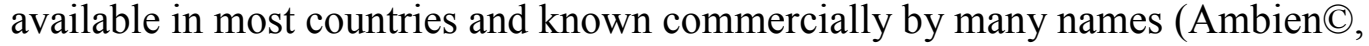
Intermezzo $\mathbb{C}$, Stilnox $($, and Zonadin(C). Zolpidem is defined by the Drug Enforcement 


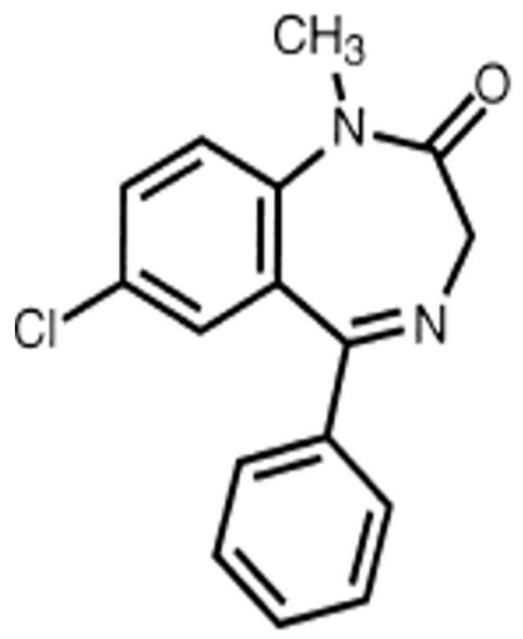

Diazepam

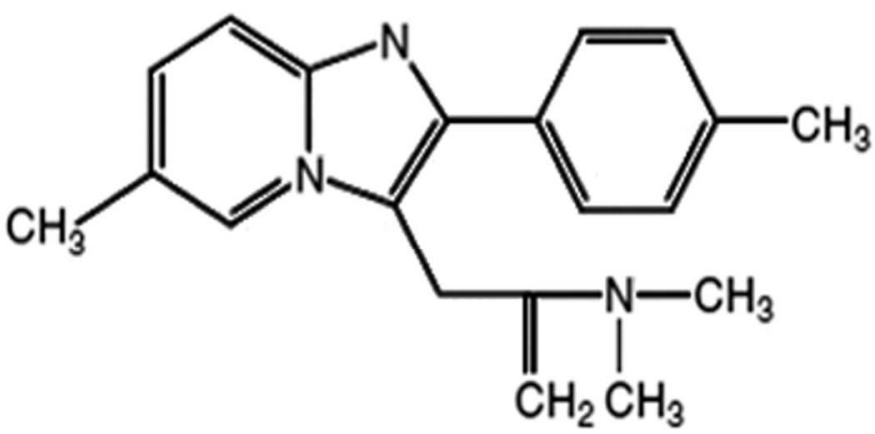

Zolpidem

Figure 2-1. Comparison of zolpidem and diazepam structures

Diazepam as a BZ is a two ring heterocyclic compound with a benzene ring fused to a diazepine ring, whereas zolpidem is an imidazopyridine which have an imidazole ring fused to a pyridine ring. Modified with permission. Drover, D.R., Comparative pharmacokinetics and pharmacodynamics of short-acting hypnosedatives: zaleplon, zolpidem and zopiclone. Clin Pharmacokinet, 2004. 43(4): p. 227-38. and Richards, J.G. and Martin, J.R., Binding profiles and physical dependence liabilities of selected benzodiazepine receptor ligands. Brain Research Bulletin. 45(4): p.381-387 [31, 32]. 
Administration (DEA) as a schedule IV drug (a drug with low potential and risk of abuse and dependence). The hemitartrate salt form of zolpidem is commonly taken as a solid pill and is commonly ingested orally, but can be administered intravenously (i.v.). Pharmacological studies indicate that zolpidem has different pharmacokinetic and pharmacodynamics properties than BZs that contribute to its efficacy as a sedativehypnotic.

\section{Pharmacokinetics}

\section{Preclinical Studies}

Pharmacokinetics describes the absorption, distribution, metabolism, and elimination of a drug from the body. The pharmacokinetics of a drug determines the onset, duration, and intensity of a drug's effect. In rodents, a 5-10 mg dose of zolpidem has a peak concentration $\left(\mathrm{C}_{\max }\right)$ of about $2341 \mathrm{ng} / \mathrm{ml}$ and a time of peak concentration $\left(\mathrm{T}_{\max }\right)$ of 5-15 min [33]. Zolpidem has a volume of distribution $\left(\mathrm{V}_{\mathrm{d}}\right)$ of $104.9 \mathrm{ml}$, no active metabolites, and has a half-life $\left(\mathrm{t}_{1 / 2}\right)$ of approximately 20-90 $\mathrm{min}$ [33-35]. In rodents, a 5-20 mg dose of diazepam has a $\mathrm{C}_{\max }$ of about $297 \mathrm{ng} / \mathrm{ml}$ and a $\mathrm{T}_{\max }$ of $72 \mathrm{~min}$ [36]. Diazepam has a $V_{d}$ of $920 \mathrm{ml}$ and a $t_{1 / 2}$ of 52 minutes [37]. Diazepam has an active metabolite, desmethyldiazepam, whose half-life is 11.1 hours and has sedative effects [37]. In rodents, zolpidem has a higher peak concentration and reaches it in a shorter amount of time compared to diazepam. Zolpidem also has a lower volume of distribution and a lower $t_{1} / 2$ than diazepam. In contrast to diazepam, the degradation of zolpidem does not lead to the accumulation of active metabolites that can sustain drug action $[31,33,38$, 39].

\section{Clinical Studies}

In humans, a $10 \mathrm{mg}$ dose of zolpidem or diazepam initiates sleep [40-42]. A 10 $\mathrm{mg}$ dose of zolpidem has a $\mathrm{T}_{\max }$ between 1.2-1.6 hours similar. A $10 \mathrm{mg}$ dose of diazepam has a $\mathrm{T}_{\max }$ between 1.3-1.4 hours, similar to zolpidem. Zolpidem has a $\mathrm{C}_{\max }$ of $120-140 \mathrm{ng} / \mathrm{ml}$ while diazepam at the same dose has a $C_{\max }$ of 253-885 $\mathrm{ng} / \mathrm{ml}$ [31, 43-45]. Zolpidem has a bioavailability (fraction of unchanged drug that reaches the systemic circulation) of about $70 \%$ after first pass hepatic metabolism with $92 \%$ bound to protein [31]. Diazepam has a bioavailability of about $100 \%$ with around $97 \%$ bound to protein $[31,43,46]$.

The main cytochrome P450 (CYP450) enzyme, isozymes responsible for the biotransformation of drugs, that metabolize zolpidem into inactive metabolites is CYP3A4 accounting for about $60 \%$ of clearance, the amount of drug removed from the body per unit time [47]. The other CYP450s that metabolize zolpidem are CYP2C9, accounting for 22\%; CYP1A2, accounting for 14\%; and CYP2D6/CYP2C19, accounting for $<3 \%$ of clearance [47]. Diazepam is metabolized by CYP2C9, 2C19, 2B6, 3A4, 3A5, 
3A4 and 2C19 into three active metabolites, desmethyldiazepam, temazepam, and oxazepam $[31,43,44]$. Zolpidem has an $t_{1 / 2}$ of about 3 hours in comparison to diazepam with a long $\mathrm{t}_{1 / 2}(\sim 100$ hours due to active metabolites) [48]. Zolpidem has a lower bioavailability and a shorter $t_{1 / 2}$ than diazepam. In comparison to diazepam's many active metabolites, zolpidem does not have active metabolites.

Recent evidence suggest that gender and age influences the pharmacokinetics of zolpidem. There is evidence that women have a lower rate of clearance of zolpidem and have higher blood plasma concentrations of zolpidem than men [49-51]. This may be due to a tempering effect of testosterone as elderly men also have a lower clearance that is correlated with reduced testosterone (a CYP3A4 substrate) in men [52]. These finding prompted the U.S. Food and Drug Administration (FDA) to recommend lowering the dose of zolpidem for women, as the dose for the elderly was already reduced [53]

\section{Pharmacodynamics}

\section{Preclinical Studies}

Pharmacodynamics is the study of the biochemical and physiological effects of drugs on the body and the relationship between drug concentration and effect. These effects include receptor affinity, receptor efficacy, and drug effect on behavior.

At clinical doses (5-10 mg), zolpidem has preferential affinity for $\alpha 1-\mathrm{GABA}_{\mathrm{A}} \mathrm{Rs}$, lower affinity for $\alpha 2$ - and $\alpha 3-\mathrm{GABA}_{\mathrm{A}} \mathrm{Rs}$, and no affinity for $\alpha 5-\mathrm{GABA}_{\mathrm{A}} \mathrm{Rs}$ [34, 54]. It has been shown that increasing the concentration of zolpidem does cause affinity to shift towards $\alpha 2$ - and $\alpha 3-G_{3} A_{A}$ Rs $[55,56]$. Diazepam $(10-20 \mathrm{mg})$ in comparison, has similar affinity at $\alpha 1-, \alpha 2-, \alpha 3-$, and $\alpha 5-G A B A_{A} R s$ [57]. Multiple in-vitro and in-vivo studies have discerned the effects of both drugs on $\mathrm{GABA}_{\mathrm{A}} \mathrm{R}$ functioning. The amount of zolpidem required to potentiate $\alpha 1-\mathrm{GABA}_{\mathrm{A}} \mathrm{Rs}$ is lower than to potentiate $\alpha 2-, \alpha 3-$, and $\alpha 5-G_{A B A}{ }_{A}$ Rs [17, 57-60]. Zolpidem at high doses $(>10 \mathrm{nM})$ enhances the amplitude of miniature inhibitory post-synaptic currents (mIPSCs), beta wave frequency (low amplitude beta waves associated with increased brain activity), and mIPSC duration in the cortex [61, 62]. At low doses $(<10 \mathrm{nM})$, zolpidem decreases the beta wave frequency [62]. Diazepam at concentrations of $10 \mathrm{nM}-1 \mu \mathrm{M}$ potentiated $\mathrm{Cl}^{-}$currents and enhanced IPSCs in HEK cells, the hippocampus, and the basolateral amygdala (BLA) and central amygdala (CeA) [63-65].

The activation or positive modulation of $\mathrm{GABA}_{\mathrm{A}} \mathrm{Rs}$ generally depresses motor behavior. Zolpidem and diazepam at doses of $1-100 \mathrm{mg} / \mathrm{kg}$ cause reductions in measures of locomotor activity, ataxia, muscle relaxant properties, and loss of righting reflex (LORR) [66-72]. In addition to depressing motor behavior, zolpidem and diazepam increases the threshold of pentylenetetrazole, electroconvulsive, and isoniazid induced convulsions $[69,70]$. Zolpidem and diazepam's effects on sleep structure and associated 
behaviors show some similarities, both increase slow wave sleep (SWS) and decrease body temperature in rodents, though diazepam causes tachycardia and hypertension [73].

Knockout $(\mathrm{KO})$ mice are mice that are missing a particular $\mathrm{GABA}_{\mathrm{A}} \mathrm{R}$ subunit. $\mathrm{KO}$ mice have been used to discover how different $\mathrm{GABA}_{\mathrm{A}} \mathrm{R}$ subtypes modulate behaviors like sedation and hypnosis. KO mice that have the $\alpha 1$ subunit deleted, show a reduced hypnotic effect compared to wild-type (no deletion of $\alpha 1$ subunit) counterparts when given zolpidem though they show increased hypnotic effect when given diazepam [18]. The $\alpha 1-G_{A B A}$ Rs mainly mediate zolpidem's hypnotic effects while $\alpha 2-\mathrm{GABA}_{\mathrm{A}} \mathrm{Rs}$ are responsible for diazepam's hypnotic effects $[18,74]$. However an issue with KO mice is that results gained from them may be due to compensatory increases in other GABA $\mathrm{A}_{\mathrm{A}}$. Another tool used to examine how different GABA $\mathrm{A}_{\mathrm{R}}$ subtypes modulate behavior are point mutation mice. The mice have a single point mutation in the $\alpha 1$-subunit protein, allowing for the receptor to be BZ and zolpidem-insensitive. Another benefit is that unlike KO mice, there are no compensatory increases in $\alpha 2, \alpha 3-, \alpha 5-\mathrm{GABA}_{\mathrm{A}} \mathrm{Rs}$ that may account for potential effects. Through the use of point mutation mice it has been found that $\alpha 1-\mathrm{GABA}_{\mathrm{A}} \mathrm{Rs}$ mediate the locomotor impairing effect (measure of sedation) and anticonvulsant effects of zolpidem and the locomotor impairing effects of diazepam [71]. This indicates $\alpha 1-\mathrm{GABA}_{\mathrm{A}}$ Rs mediate zolpidem's sedative and hypnotic effects and partially mediate diazepam's sedative effects as well [71]. Since $\alpha 1-\mathrm{GABA}_{\mathrm{A}} \mathrm{Rs}$ modulation is important for sedation and hypnosis, it is possible that it also plays a part in the development of tolerance and withdrawal to these behaviors.

\section{Clinical Studies}

As in rodents, zolpidem has a greater binding affinity for $\alpha 1-\mathrm{GABA}_{\mathrm{A}} \mathrm{Rs}[75,76]$. Diazepam shows equal affinity for $\alpha 1-, \alpha 2-, \alpha 3-$, and $\alpha 5-\mathrm{GABA}_{\mathrm{A}} \mathrm{Rs}$ with human recombinant $\mathrm{GABA}_{\mathrm{A}} \mathrm{Rs}[17,77,78]$. Both diazepam and zolpidem positively modulate $\alpha 1-, \alpha 2-$, and $\alpha 3-\mathrm{GABA}_{\mathrm{A}}$ Rs with maximum potentiation $>70 \%$ [78]. Zolpidem has a $\mathrm{GABA}_{\mathrm{A}} \mathrm{R}$ occupancy in humans of about $27 \%$ in the neocortex indicating that zolpidem is effective at low occupancy [75].

Zolpidem at clinical doses ( 5 and $10 \mathrm{mg}$ ) exerts a hypnotic effect by shortening the latency to sleep, maintaining non-rapid eye movement (NREM) sleep, and increasing sleep duration in humans $[31,79]$. Zolpidem is highly effective in initiating sleep, but is not as effective in maintaining sleep. In humans zolpidem induces sedation, impairs memory and psychomotor performance $[49,50,80,81]$. Zolpidem seems to have increased adverse effects when compared to hypnotic alternatives like zaleplon, but lower adverse effects than diazepam [80, 82]. Although zolpidem is mainly used as a hypnotic, it also has weak anxiolytic, anticonvulsant, and amnesic effects. In humans zolpidem has weak anticonvulsant activity against pentylenetetrazole (PTZ) and electroshock convulsions in a dose dependent manner [69]. Intraseptal injections of zolpidem impairs spatial working memory and induces retrograde memory deficits [83]. Acute zolpidem injections also impair associative and contextual memory [84-86]. Diazepam but not zolpidem increases inhibition in the human motor cortex despite sedation by both drugs 
as measured by short interval intracortical inhibition (SICI; a GABA $\mathrm{A}_{\mathrm{A}}$ mediated inhibition in the motor cortex) [87].

\section{GABAAR Overview}

\section{GABAergic System}

GABA is the main inhibitory neurotransmitter of the CNS. GABA binds to two different types of receptors, $\mathrm{GABA}_{\mathrm{A}} \mathrm{Rs}$ and $\mathrm{GABA}_{\mathrm{B}}$ Rs. $\mathrm{GABA}_{\mathrm{A}} \mathrm{Rs}$ are ligand gated chloride $\left(\mathrm{Cl}^{-}\right)$ion channels (transmembrane channels that facilitate the passage of ions through a membrane when a ligand binds to the receptor) and $\mathrm{GABA}_{\mathrm{B}} \mathrm{Rs}$ are metabotropic receptors (a receptor that acts through secondary messengers). The GABA ${ }_{A} \mathrm{R}$ (Figure 2-2) facilitates the passage of $\mathrm{Cl}^{-}$ions, hyperpolarizing neuron [8891]. The $\mathrm{GABA}_{\mathrm{A}} \mathrm{R}$ is heteropentameric (a receptor made up of five subunits) with 19 known subunits. The subunits, $\alpha 1-6, \beta 1-3, \gamma 1-3, \rho 1-3, \delta, \varepsilon, \theta$, and $\pi$, can occupy the five positions of the $\mathrm{GABA}_{\mathrm{A}} \mathrm{R}$ [88]. The GABA $\mathrm{R}$ is commonly composed of two $\alpha$ subunits, two $\beta$ subunits, and a $\gamma$ subunit. GABA ${ }_{A}$ Rs composed of two $\alpha 1$ subunits, two $\beta 2$ subunits, and a $\gamma 2$ subunit represent about $60 \%$ of the $\mathrm{GABA}_{\mathrm{A}} \mathrm{Rs}$ in the rodent brain and are widely expressed in the brain [90, 92-94]..

\section{GABA $_{A} R$ Subunit Localization and Distribution}

The $\alpha 1-, \alpha 2-$, and $\alpha 3-\mathrm{GABA}_{\mathrm{A}} \mathrm{Rs}$ are often located directly opposite the presynaptic terminal and are referred to as synaptic receptors based on this localization. Synaptic $\mathrm{GABA}_{\mathrm{A}} \mathrm{Rs}$ modulate phasic (transient/fast) inhibition $(<500 \mu \mathrm{secs})$ in the presence of high concentrations $(>50 \mathrm{uM})$ of GABA released into the synapse. The $\alpha 4-$, $\alpha 5$, and $\alpha 6-\mathrm{GABA}_{\mathrm{A}} \mathrm{Rs}$ are mostly located outside the synapse and are referred to as extrasynaptic receptors based on this localization. Extra-synaptic $\mathrm{GABA}_{\mathrm{A}} \mathrm{Rs}$ modulate tonic (persistent/slow) inhibition $(>500 \mu \mathrm{secs})$ in the presence of low concentrations $(<50$ $\mathrm{uM}$ ) of GABA that diffuse outside the synapse (Figure 2-3) [92, 95].

The distribution of $\mathrm{GABA}_{\mathrm{A}} \mathrm{R}$ subunit $\mathrm{mRNAs}$ and proteins vary from region to region in the mouse brain (Figure 2-4) $[50,96,97]$. The distribution of GABA $A$ subunits in the mouse brain show that the cortex and hippocampus contain most of the subunits with the exception of $\alpha 6$ and $\gamma 1$ [93]. The amygdala contains a majority of the subunits with the exception of $\alpha 6$ [93]. The cortex has high levels of $\alpha 1, \alpha 2, \alpha 3, \beta 2, \beta 3$, and $\gamma 2$ subunits. The hippocampus has high levels of $\alpha 1$, and $\beta 2$ subunits, and very high levels of $\alpha 2, \alpha 5, \beta 3$, and $\gamma 2$ subunits. The amygdala has high levels of $\alpha 2, \alpha 3, \beta 3$, and $\gamma 2$ subunits with high levels of the $\alpha 1$-subunit mRNA expression in the BLA [94]. 


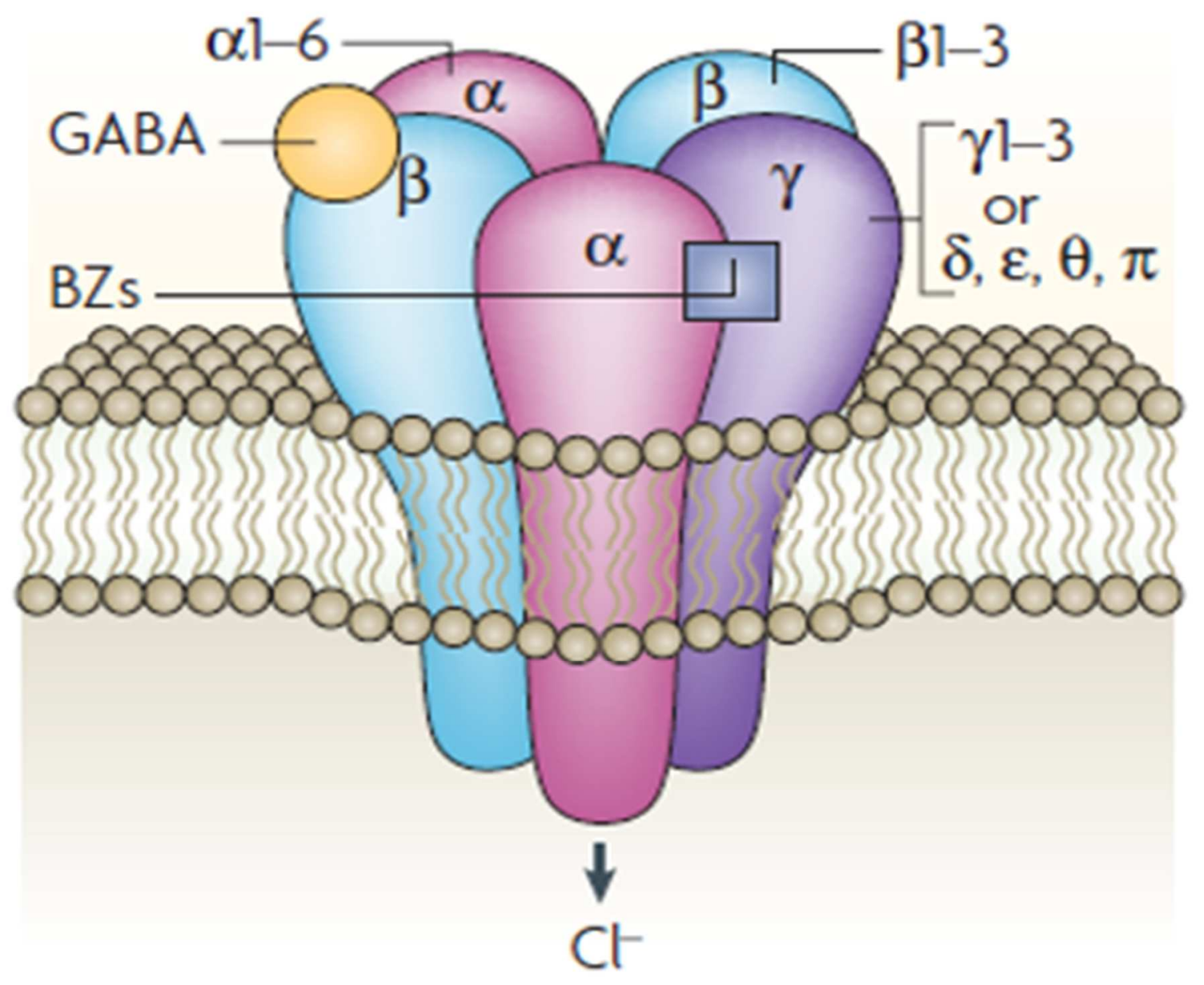

\section{Figure 2-2. Example of a typical $G_{A B A_{A}} R$ complex}

The $\mathrm{GABA}_{\mathrm{A}} \mathrm{R}$ is an ion channel that allows for entry of the $\mathrm{Cl}$ ion composed of 5 subunits. $\mathrm{GABA}_{\mathrm{A}}$ Rs are usually composed of $2 \alpha$ subunits, $2 \beta$ subunits and a $\gamma$ subunit with the interface between $\alpha$ and $\beta$ subunits contain a GABA binding site and the interface of $\alpha$ and $\gamma$ subunits contains the BZ/non-BZ binding site. Reprinted with permission. Jacob, T.C., S.J. Moss, and R. Jurd, GABA(A) receptor trafficking and its role in the dynamic modulation of neuronal inhibition. Nat Rev Neurosci, 2008. 9(5): p. 33143 [91]. 


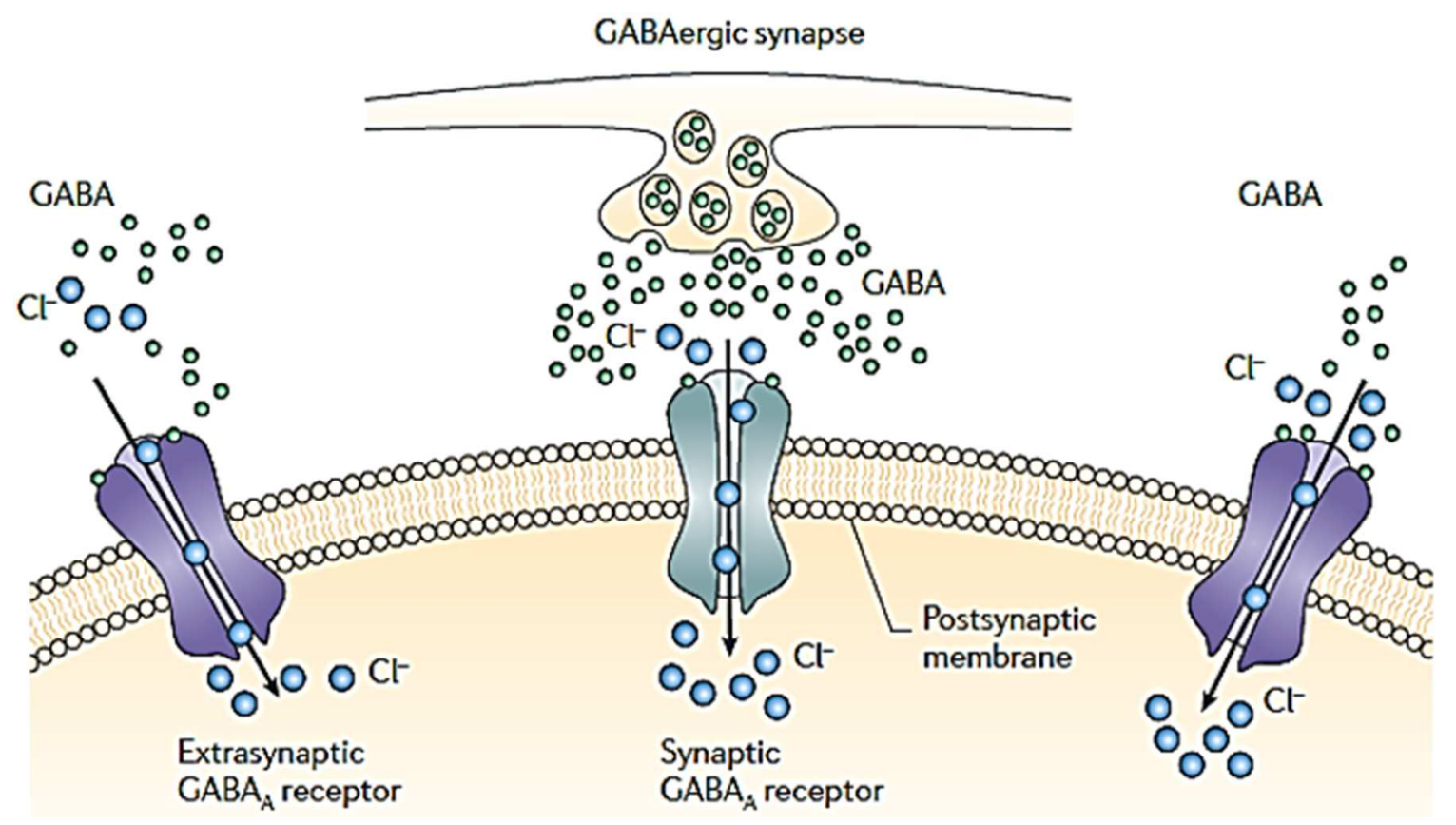

Figure 2-3. Example of the localization of synaptic and extrasynaptic $G A B A_{A} R$ opposite the presynaptic cleft

At the GABAergic synapse presynaptic vesicles release GABA onto synaptic and extrasynaptic $\mathrm{GABA}_{\mathrm{A}}$ receptors. Reprinted with permission Rudolph, U. and F. Knoflach, Beyond classical benzodiazepines: novel therapeutic potential of GABAA receptor subtypes. Nat Rev Drug Discov, 2011. 10(9): p. 685-97 [90]. 

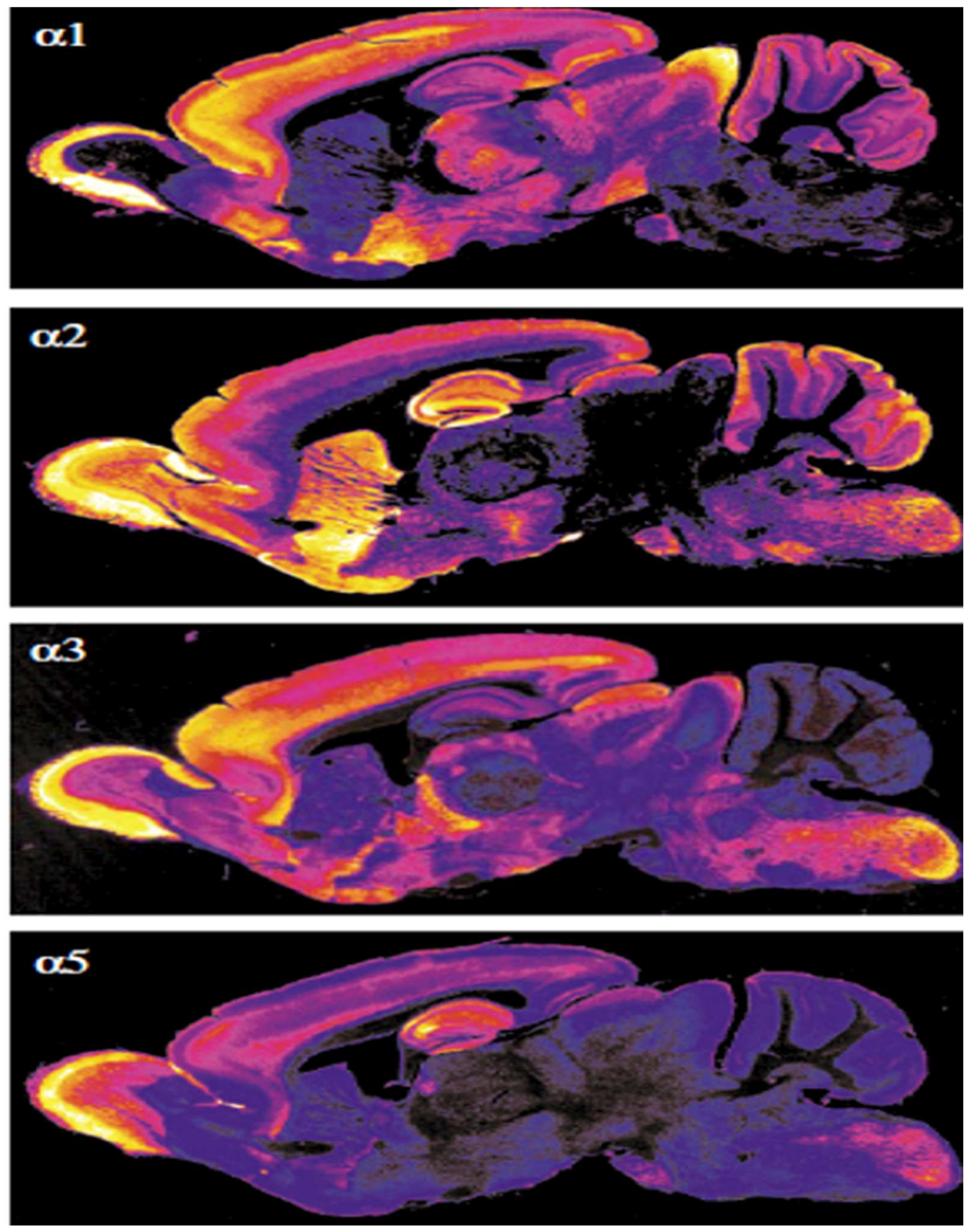

Figure 2-4. Example of differential regional distribution of $\alpha$ subunits proteins in rat brain sensitive to $\mathrm{BZs}$

The $\alpha 1$ subunit is expressed in most brain regions with high expression in the cortex. The $\alpha 2$ subunit has high expression in the hippocampus and amygdala. The $\alpha 3$ subunit has high expression in the amygdala, brain stem, basal forebrain and the thalamus. The $\alpha 5$ subunit is mainly expressed in the hippocampus. Reprinted with permission. Rudolph, U., F. Crestani, and H. Mohler, GABA(A) receptor subtypes: dissecting their pharmacological functions. Trends Pharmacol Sci, 2001. 22(4): p. 188-94 [97]. 


\section{GABAAR Subtypes and Modulatory Effects}

$\mathrm{GABA}_{\mathrm{A}}$ Rs subtypes modulate various behaviors. The positive modulation of $\alpha 1$ $\mathrm{GABA}_{\mathrm{A}}$ Rs mainly cause sedation, hypnosis, partial anticonvulsant effects, anterograde amnesia and addiction $[71,90,98]$. The $\alpha 2-$ and $\alpha 3-\mathrm{GABA}_{\mathrm{A}} \mathrm{Rs}$ mainly mediate anxiety and myorelaxation [98]. Most BZs bind to $\alpha 1-, \alpha 2-, \alpha 3-$, and $\alpha 5-\mathrm{GABA}_{\mathrm{A}} \mathrm{Rs}$, while zolpidem binds preferentially to $\alpha 1-\mathrm{GABA}_{\mathrm{A}} \mathrm{Rs}$, has some affinity for the $\alpha 2$ - and $\alpha 3$ $\mathrm{GABA}_{\mathrm{A}} \mathrm{Rs}$, and no affinity to the $\alpha 5-\mathrm{GABA}_{\mathrm{A}} \mathrm{Rs}$ [99].

\section{Contributions of GABA and Glutamate mRNA and Protein Alterations in the Cortex, PFC, Hippocampus, and Amygdala to Sedative Tolerance and Withdrawal Symptoms}

Although it is known that both BZ and zolpidem can induce tolerance in mice, it is unclear whether specific alterations in brain regions mediate tolerance because the alterations after repeated BZ and zolpidem on brain regions are inconsistent. While BZs and zolpidem affect many brain regions, this project is focusing on the cortex, which modulates locomotion as well as the PFC, hippocampus, and amygdala. As stated above the cortex, hippocampus, amygdala, and PFC have high expressions of BZ-sensitive $\mathrm{GABA}_{\mathrm{A}}$ Rs which may play a role in tolerance and withdrawal, particularly withdrawalinduced anxiety $[87,94,100,101]$. Symptoms of tolerance and withdrawal have also been associated with changes in glutamatergic receptor systems [102-104]. At present, there are few studies that measure the effects of repeated zolpidem on GABA $\mathrm{R}$ and glutamatergic receptors, however studies that have examined the effects of repeated BZ can shed light on the potential changes after zolpidem use.

There are inconsistencies in the changes caused by BZs and zolpidem treatment. One consistent finding is that repeated $\mathrm{BZ}$ and zolpidem reduced the expression of $\alpha 1$ $\mathrm{GABA}_{\mathrm{A}} \mathrm{R}$ subunit mRNA and protein and increased the expression of $\alpha 4 \mathrm{GABA}_{\mathrm{A}} \mathrm{R}$ subunit mRNA and protein in the cortex, although some studies do not see these changes [105-114]. However some studies have found that in the rat cortex after diazepam treatment ( 7 and 14 days) there were no significant differences in $\alpha 1$-subunit mRNA, but increases in $\alpha 3-, \alpha 4-$, and $\alpha 5$-subunit mRNA $[107,111,115,116]$. There were no changes in the $\beta 2$-subunit but there was a decrease in $\gamma 2$ - subunit mRNA in the cortex [111]. Repeated BZ and zolpidem also reduce $\alpha 1 \mathrm{mRNA}$ and protein expression in the PFC [105]. Diazepam treatment caused an increase in the $\alpha 5$-subunit in the PFC [105]. In the hippocampus, repeated diazepam has caused a decrease in in $\alpha 1-$ and $\alpha 5$-subunit mRNA $[105,107,113,116]$. In the cortex, diazepam withdrawal also reportedly caused an increase in GluR1, GluN1, and GluN2B subunit mRNA [104, 117]. In the hippocampus, BZ withdrawal induced increases in GluR1, GluN1, and GluN2B subunit mRNA and protein and increased AMPAR responses [102, 118, 119]. In addition, diazepam withdrawal caused a decrease in GluR1 and GluR2 subunit mRNA expression in the amygdala [53, 117]. 
The changes found in all of the regions reflect an imbalance in the excitatory and inhibitory tone. A reduction of $\mathrm{BZ}$-sensitive $\mathrm{GABA}_{\mathrm{A}} \mathrm{Rs}$ seem to be more related to the development of tolerance, while the upregulation of AMPARs and NMDARs in these regions appear to be more associated with withdrawal induced regulation.

\section{Adverse Consequences of Zolpidem Use}

\section{Tolerance}

Preclinical Studies. BZs are known to induce dependence in rodents and primates [120]. In rodent and primate models dependence is usually manifested as tolerance and withdrawal. As defined in the Diagnostic and Statistical Manual of Mental Disorders, $5^{\text {th }}$ Edition (DSM-V), tolerance is operationally defined as a loss of efficacy to a given effect at a given dose or the need for higher doses for the same effect [1]. Tolerance due to chronic BZ treatment is well documented in rodents [109, 112, 120 131]. A main BZ that is used to investigate sedative tolerance is diazepam. Chronic diazepam treatment results in a robust display of sedative tolerance presented as decreased sensitivity to its own locomotor-impairing effects $[119,123,124,132]$. However, there is conflicting evidence concerning the development of tolerance to chronic zolpidem in mice, with more evidence of dependence occurring in primates [24, $38,133]$. The variability in the development of tolerance may be affected by the type of behavior measured $[28,112]$. Earlier rodent studies found that tolerance did not develop to zolpidem's sedative effects [24]. Some rodent studies do not show that zolpidem can cause tolerance to the anticonvulsant and motor-sedative effects while others show tolerance to the ataxic and hypothermic effects $[29,38]$. Recent studies show that tolerance to zolpidem's sedative effects can occur $[25,105]$. In addition to tolerance to its own effects zolpidem shows cross-tolerance, development of tolerance to a drug that causes tolerance to develop to another drug, to other BZs. It has been found that mice previously treated with 28 days of zolpidem were tolerant to the effects of diazepam's hypothermic effect, with partial tolerance to the anxiolytic effect, and no tolerance to the sedative effect [112]. Zolpidem had a decreased potency to induce locomotor impairment and antagonize isoniazid-induced convulsions in mice treated with 10 days of midazolam [24].

Clinical Studies. In human and non-human primates chronic, use of zolpidem has been shown to lead to dependence accompanied by withdrawal, though there are some studies that find zolpidem maintains a low abuse potential in humans [26, 134-138]. Many human case studies show common themes between the patients that abuse zolpidem. These themes are tolerance and withdrawal (Table 2-1) [26, 139, 140].

Patients who become dependent on zolpidem tend to use zolpidem at supratherapeutic doses $(>20 \mathrm{mg})$; though there are those who maintain dependence 
Table 2-1. Case reports on dependence/abuse of zolpidem

\begin{tabular}{|c|c|c|c|c|c|c|c|c|c|c|}
\hline No. & $\begin{array}{l}\text { Age } \\
\text { (years) }\end{array}$ & Gender & $\begin{array}{l}\text { Highest daily } \\
\text { dose (mg/day) }\end{array}$ & $\begin{array}{l}\text { Daytim- } \\
\text { e Intake }\end{array}$ & $\begin{array}{l}\text { Dose } \\
\text { increase }\end{array}$ & Tolerance & $\begin{array}{l}\text { Withdrawal } \\
\text { symptoms }\end{array}$ & $\begin{array}{l}\text { Former } \\
\text { dependence }\end{array}$ & $\begin{array}{l}\text { Psychiatric } \\
\text { complicati- } \\
\text { ons }\end{array}$ & Reference \\
\hline 1 & 67 & $\mathrm{~F}$ & 100 & + & + & + & + & + & + & Madrak \& Rosenberg 2001 \\
\hline 2 & 43 & $\mathrm{~F}$ & $450-600$ & - & + & + & + & - & $\mathrm{nr}$ & Aragona 2000 \\
\hline 3 & 39 & M & $120-480 b$ & $\mathrm{nr}$ & + & $\mathrm{nr}$ & + & - & + & Golden \& Vagnoni 2000 \\
\hline 4 & 42 & $\mathrm{~F}$ & 300 & $\mathrm{nr}$ & + & + & + & + & + & Vartzpopulos et al. 2000 \\
\hline 5 & 30 & $\mathrm{~F}$ & $400-500$ & $\mathrm{nr}$ & + & + & + & + & + & Vartzpopulos et al. 2000 \\
\hline 6 & 26 & $\mathrm{~F}$ & $160-200$ & $\mathrm{nr}$ & + & + & + & + & + & Vartzpopulos et al. 2000 \\
\hline 7 & 33 & M & 120 & $\mathrm{nr}$ & + & + & + & + & + & Vartzpopulos et al. 2000 \\
\hline 8 & 45 & F & $\begin{array}{l}40 \\
\text { (+zopiclone) }\end{array}$ & + & + & + & + & - & + & Harter $1999^{\mathrm{a}}$ \\
\hline 9 & 49 & M & $50-80$ & + & + & + & + & - & - & Ströhle 1999 \\
\hline 10 & 50 & $\mathrm{~F}$ & $=600$ & + & + & + & + & + & + & Hofmann \& Eichmann 1998 \\
\hline 11 & 44 & $\mathrm{~F}$ & $30-300$ & + & + & + & + & $\mathrm{nr}$ & + & Sakkas 1999 \\
\hline 12 & 63 & F & 300 & + & + & + & + & $\mathrm{nr}$ & $?$ & Courtet 1999 \\
\hline 13 & 28 & $\mathrm{~F}$ & 800 & - & + & - & + & $\mathrm{nr}$ & $?$ & Courtet 1999 \\
\hline 14 & 69 & $\mathrm{~F}$ & 200 & + & + & + & - & $\mathrm{nr}$ & $?$ & Courtet 1999 \\
\hline 15 & 32 & M & 60 & + & + & + & - & + & $?$ & Courtet 1999 \\
\hline 16 & 45 & M & 120 & + & + & + & + & $\mathrm{nr}$ & $?$ & Courtet 1999 \\
\hline 17 & 40 & M & 200 & + & + & $\mathrm{nr}$ & $\mathrm{nr}$ & + & $?$ & Courtet 1999 \\
\hline 18 & 35 & M & 100 & + & + & $\mathrm{nr}$ & $\mathrm{nr}$ & $\mathrm{nr}$ & $?$ & Courtet 1999 \\
\hline 19 & 55 & $\mathrm{~F}$ & $=200$ & $\mathrm{nr}$ & + & + & + & $\mathrm{nr}$ & + & Ravishankar 1998 \\
\hline 20 & 28 & M & $=100$ & $\mathrm{nr}$ & + & $\mathrm{nr}$ & + & $\mathrm{nr}$ & + & Ravishankar 1998 \\
\hline 21 & 53 & M & 140 & + & + & + & + & + & - & Bottlender 1997 \\
\hline 22 & 37 & M & 130 & + & + & + & + & - & - & Gilbert \& Stats 1997 \\
\hline 23 & 35 & M & $200-300$ & - & + & + & + & - & + & Chamorro-Garcia 1996 \\
\hline 24 & 33 & M & $300-400$ & + & + & + & + & - & $\mathrm{nr}$ & Sanchez 1996 \\
\hline 25 & 75 & M & 70 & + & + & + & + & - & $\mathrm{nr}$ & Thome 1995 \\
\hline 26 & 33 & M & $150-280$ & + & + & + & + & - & + & Gericke 1994 \\
\hline 27 & 42 & $\mathrm{~F}$ & $\mathrm{nr}$ & $\mathrm{nr}$ & $\mathrm{nr}$ & $\mathrm{nr}$ & + & + & + & Bruun 1993 \\
\hline 28 & 52 & M & $60-70$ & $\mathrm{nr}$ & + & + & + & + & + & Bruun 1993 \\
\hline 29 & 55 & $\mathrm{~F}$ & $=120$ & + & + & + & + & + & $\mathrm{nr}$ & Bruun 1993 \\
\hline 30 & 60 & F & 100 & $\mathrm{nr}$ & + & + & + & + & + & Cavallaro 1993 \\
\hline 31 & 31 & F & $70-80$ & + & + & + & + & $\mathrm{nr}$ & $\mathrm{nr}$ & Cavallaro 1993 \\
\hline 32 & 67 & $\mathrm{~F}$ & 100 & $\mathrm{nr}$ & + & + & + & - & + & Göder et al. 2001 \\
\hline 33 & 50 & $\mathrm{~F}$ & 450 & + & + & $\mathrm{nr}$ & + & - & $\mathrm{nr}$ & $\begin{array}{l}\text { Barrero-Hernandez et al. } \\
2002\end{array}$ \\
\hline 34 & 51 & M & 1200 & + & + & $\mathrm{nr}$ & + & + & $\mathrm{nr}$ & Correas Lauffer et al. 2002 \\
\hline 35 & 46 & $\mathrm{~F}$ & 200 & $\mathrm{nr}$ & + & $\mathrm{nr}$ & + & + & + & Correas Lauffer et al. 2002 \\
\hline 36 & 40 & $\mathrm{~F}$ & 200 & + & + & $\mathrm{nr}$ & + & + & + & Correas Lauffer et al. 2002 \\
\hline
\end{tabular}

$\mathrm{M}=$ male, $\mathrm{F}=$ female; $\mathrm{nr}=$ not reported. ${ }^{\mathrm{a} C o n c o m i t a n t}$ abuse of zopiclone and zolpidem.

${ }^{\mathrm{b}} 10-40$ quaque hora somni (qhs; every hour of sleep).

The use of doses outside the therapeutic range $(>10 \mathrm{mg} / \mathrm{kg})$, increase in dose over time, tolerance, withdrawal, as well as usage during the daytime are common in those who abuse zolpidem. Reprinteda nd modified with permission. Hajak, G., et al., Abuse and dependence potential for the non-benzodiazepine hypnotics zolpidem and zopiclone: a review of case reports and epidemiological data. Addiction, 2003. 98(10): p. 1371-8 [26]. 
below this dose) $[26,135,141]$. Another common theme are that those who become dependent previously abused BZs, opioid, and alcohol, or had psychiatric disorders [26]. Though some studies looking at extended use of zolpidem do not find escalation or tolerance to zolpidem's sedative effects in insomniacs and "normal" patients [40].

\section{Withdrawal}

Preclinical Studies. As defined in the DSM-V, withdrawal is the typical effects associated with the sudden stoppage of a drug[1]. For BZs and zolpidem the withdrawal syndrome is displayed as hyper-excited activity, like increased anxiety and insomnia and in severe cases seizures and hallucinations. Tolerance and withdrawal tend to be associated with each other, but they do not always occur together. Tolerance and withdrawal may have similar mechanisms. Withdrawal effects in rodents and primates can take many forms. Rats given chronic lorazepam experienced spontaneous and precipitated withdrawal, measured by motor activity within the home cage and increased electromyography (EMG) activity compared to control [38]. Chronic zolpidem compared to lorazepam did not display withdrawal symptoms [38]. Increased precipitated withdrawal scores (PAS) which include convulsions, motor signs, dyskinesia, autonomic signs, and affective signs were seen after chronic diazepam [142]. Mice given chronic diazepam and subjected to either repeated withdrawals or a single withdrawal, have a lower dose threshold for pentylenetetrazole (PTZ) after flumazenil precipitated withdrawal [143]. Many studies have found that BZs induce hyperactivity and anxietylike effects in rodents. Of note is the duration between cessation of BZ treatment and the development of withdrawal symptoms. Some studies identify symptoms within 24 hours of drug termination while others saw symptoms after 96 hours [104]. Other studies have found that mice treated with zolpidem do not have clonic seizures when given the $\beta$ carboline inverse agonist FG-7142 after 4 days of treatment [144].

Clinical Studies. Initially zolpidem was thought to be absent of negative effects. Emerging case studies have found that patients increase zolpidem dosage beyond clinical doses. Once these patients stop taking these high doses, withdrawal occurs. Human withdrawal from BZs are well documented and tend to be severe, as dosage increases resulting in severe insomnia, seizures, anxiety, sweating, tremors, and irritation [139, $145,146]$. Patients tend to take supratherapeutic doses of these drugs due to the prescribed doses losing efficacy (tolerance) or to prevent withdrawal symptoms [30].

\section{Relationship Between Duration of Zolpidem Use and the Emergence of Tolerance and Withdrawal}

Most controlled studies investigating the effects of repeated zolpidem use in humans have not found tolerance and withdrawal. These studies allowed participants to self-administer and did not show an increase in dose level across time, suggesting 
tolerance to the sedative effects do not occur. The studies also found there were no withdrawal effects such as next day insomnia or cognitive dysfunction [27, 40]. These data indicate zolpidem is a safe drug with a low propensity for tolerance and withdrawal. However, most of these controlled studies were done in healthy adults with or without insomnia and with no prior BZ, opioid, or alcohol abuse. Unlike controlled studies, case studies have recorded numerous cases of zolpidem dependency primarily in patients with prior BZ, opioid, or alcohol abuse and psychiatric disorders. The amount of time it takes these patients to become tolerant to zolpidem varies. Usually patients become tolerant within 1-6 months of starting zolpidem, increasing the dose substantially from $10 \mathrm{mg}$ to $>$ $100 \mathrm{mg}[147,148]$. The amount of time between cessation of zolpidem and the emergence and severity of withdrawal symptoms also varies. In humans mild withdrawal symptoms, like anxiety and tremors occurs within hours of the last dose [149]. More severe withdrawal symptoms (e.g. seizures and hallucinations) can occur up to 3 days after the last dose of zolpidem [139, 145]. It should be noted that these symptoms usually occur after cessation of extremely high doses of zolpidem that taken for up to two years.

Along with humans, non-human primates can display tolerance to zolpidem. Interestingly primates show tolerance to the sedative effects of zolpidem at relatively low doses of 3.2 and $5.6 \mathrm{mg} / \mathrm{kg}$ over the course of 7 days of treatment [138]. Non-human primates also reliably show withdrawal symptoms after the abrupt end of zolpidem treatment. At increasing doses from 1-32 $\mathrm{mg} / \mathrm{kg}$, baboons showed withdrawal symptoms after abrupt end of zolpidem treatment after 78 days of treatment [6]. Acute flumazenil given 35 days after continuous zolpidem self-injections also induced physical symptoms typically seen after BZ withdrawal in baboons, including decreased eating, increased aggression, tremors, vomiting, and abdominal discomfort [150]. The termination of longterm zolpidem administration ( $>60$ days) also caused spontaneous withdrawal symptoms comparable to BZs and barbiturates [133, 150].

In comparison to humans and non-human primates, rodents can become tolerant to zolpidem and BZs after shorter treatment durations. Rodents can become tolerant to the sedative effects of BZs as early as 1-3 days of treatment at doses of 5-20 mg/kg [122, $127,130,131,151]$. Rodents can also exhibit mild withdrawal symptoms similar to humans 1-3 days after cessation of BZs [104, 144]. Some studies show at doses of 5-10 $\mathrm{mg} / \mathrm{kg}$, rodents become tolerant to zolpidem after 7-14 days of repeated zolpidem compared to humans after $>1$ month at doses greater than $10 \mathrm{mg}$ [25, 29, 105]. However, unlike repeated zolpidem use in humans and primates, there are few studies that examine zolpidem withdrawal, with only one study reporting withdrawal symptoms after cessation of zolpidem [24, 38, 144, 152].

\section{Usefulness of Rodent Models of Sedation and Limitations}

Rodent models of sedation are useful tools to understand the neuromechanisms underlying sedation in humans. These models are valuable due to the ability to control factors that might underlie tolerance in humans. In addition, rapid development of

tolerance can be achieved in rodents compared to human and primate. As stated above, 
rodents can become tolerant to zolpidem after 7 days of drug treatment compared to humans, which take $>1$ month to become tolerant to the effects of zolpidem. While the rodent model of sedation may be a useful tool to study zolpidem tolerance, they may be limited in detecting withdrawal symptoms due to species differences in the pharmacokinetics and pharmacodynamics of zolpidem, in addition to the differential distribution of $\mathrm{GABA}_{\mathrm{A}} \mathrm{Rs}$ in the brain.

\section{Abuse, Dependence, and Addiction}

Preclinical Studies. Few studies evaluate the addictive properties of zolpidem in contrast to the many studies evaluating the addictive properties of BZs $[153,154]$. The primary measure used to evaluate addictive behavior in primates and rodents is selfadministration. Reinforcement studies have found that primates and rodents selfadminister BZs, but not at the rate of abusive drugs like cocaine [155-159]. Due to the sedative effects of BZs at higher doses, an inverted U-shaped response curve is seen. The U-shaped curve shows that response rates (function of reinforcement) as dose increase but there is a downturn in response rate due to the locomotor-impairing effects of higher doses.

Unfortunately, it is difficult to discern between physical dependence and addiction, as both tend to accompany one another. Physical dependence is characterized by the body's adaption to a given drug while addiction is the compulsive need for a drug. It has been found however that dependence can occur without reinforcement [160]. Most addictive drugs increase glutamatergic transmission in the mesolimbic dopamine system. Dependence and abuse have been behaviorally demonstrated, as baboons self-administer zolpidem indicating abuse potential but this has not been found in rodents $[133,138,150$, 161, 162]. Zolpidem has similar rates of self-injection to barbiturates and cocaine and has a higher rate of self-injection than triazolam $[138,162]$.

Abuse and dependence in mice are related and usually measured after discontinuation of drug treatment. In mice intracranial self-stimulation (ICSS) and conditioned place preference can be used as measures of a drug's reinforcing effects. BZs and other drugs of abuse reduce the amount of stimulation required for ICSS [163, 164]. It has been found that diazepam but not zolpidem dose dependently reduced reward threshold for ICSS in C57BL/6J mice [165]. This reward enhancing effect was also found in $\alpha 1(\mathrm{H} 101 \mathrm{R})$ mice, but not $\alpha 2(\mathrm{H} 101 \mathrm{R})$ or $\alpha 3(\mathrm{H} 101 \mathrm{R})$ mice, indicating that $\alpha 2-$ and $\alpha 3-$ $\mathrm{GABA}_{\mathrm{A}} \mathrm{Rs}$ play more of a role than $\alpha 1-\mathrm{GABA}_{\mathrm{A}} \mathrm{Rs}$ in the rewarding effects of BZs. Conditioned place preference (CPP), a paradigm where rodents develop a preference for one of two distinctive compartments that was previously associated with the BZs, has also been used to identify the reinforcing effects of BZs, but not zolpidem $[161,166]$.

Though studies suggests that $\alpha 1-\mathrm{GABA}_{\mathrm{A}} \mathrm{Rs}$ may not mediate rewarding effects in rodents, recent studies challenge this view. A single dose of zolpidem and diazepam increase firing of mesolimbic dopamine neurons via modulation of $\alpha 1-G A B A_{A} R s$ in the 
ventral tegmental area (VTA) [167]. Wild-type but not $\alpha 1$ (H101R) mice develop a preference for a sucrose solution with midazolam indicating a role of $\alpha 1-G A B A_{A} R s$ in the reinforcing effects of BZs $[168,169]$. Though there may be a partial role of $\alpha 1$ $\mathrm{GABA}_{\mathrm{A}} \mathrm{Rs}$ in the rewarding effects of BZs, there are no studies that show zolpidem alone produces these effects in mice. The differences in rewarding effects between rodents and primates may be due to the differences in $\mathrm{GABA}_{\mathrm{A}}$ Rs subtypes and/or binding characteristics [76]. Primate studies raise concerns about the possible rewarding effects of zolpidem and the potential for the development of abuse in humans [140]. Drugs with positive reinforcing effects have been implicated as causes for abuse and dependence, thus more research into the rewarding effects of zolpidem may provide insights into the neural mechanisms underlying drug abuse by other drugs acting through GABA $\mathrm{RS}$ [170].

Clinical Studies. Several agencies state that along with BZs, zolpidem is a highly prescribed drug of abuse [171]. In addition, there has been a 136\% increase in emergency room visits from 2004 and 2011 due to nonmedical usage of zolpidem [172]. According to the World Health Organization it is thought that abuse of zolpidem is similar in rate to other hypnotic BZs, though some studies report it as significantly lower [26, 134, 135 , 173]. Like hypnotic BZs, there appears to be some propensity for abuse among humans and baboons [133, 135-138]. Griffith and Weerts' review [6] focusing on human over usage of BZs found that those who used for more than 4 months tend to fall into two categories: recreational users and "quasi-therapeutic" users. Those who used BZs as hypnotics tend to make up $23 \%$ of BZ users yet account for the majority of sales of hypnotics. Such long-term usage may indicate a propensity for abuse that is marked by dependence, tolerance, and possibly withdrawal.

Zolpidem's propensity for abuse has been recorded through case and clinical studies. A similar characteristic of those who tend to abuse zolpidem is their prior abuse of other BZs and/or opioids [145, 174]. The cases of zolpidem abuse usually contain a withdrawal syndrome. This syndrome comes after abrupt ending of zolpidem at doses from $20 \mathrm{mg}$ to $2000 \mathrm{mg}$ a day. The sudden disuse of zolpidem at these doses result in anxiety, sweating, palpitations, seizures [139, 145, 147]. Drug naïve humans show similar potential to abuse to both zolpidem and triazolam, with zolpidem at its highest dose (20mg) increasing feelings associated with abuse potential like happy and good effects [135]. Another study with drug naïve humans found a similar profile with zolpidem at its highest dose $(20 \mathrm{mg})$ increasing feelings associated with abuse, such as high, like, and good effects [137]. Most case studies define this abuse of zolpidem as dependence not addiction. There is a case in which zolpidem displayed addictive properties such as intense craving and drug seeking to the point of harming themselves to attain the drug $[146,148]$. Along with the negative effects associated with abuse many of these studies state that patients abuse zolpidem for stimulation, euphoria, and relaxation properties [146, 148, 175]. 


\section{Side Effects/Toxicology}

Like most sedatives zolpidem has side effects that interfere with next day performance. Zolpidem in rodents has amnesic effects on a variety of memory tasks like passive avoidance (1-2 mg/kg), contextual fear conditioning (1, 3, and $10 \mathrm{mg} / \mathrm{kg})$, and the plus maze discriminative avoidance task (PM-DAT) [85, 176, 177].

There are few incidences of toxicity in humans at therapeutic doses and fewer instances of overdose compared to BZs. A review of zolpidem's side effects found they come in the form of lightheadedness, dizziness, somnolence, headache, fatigue, memory deficits, nightmares, confusion, and depression, nausea, and vomiting [178-180]. In some instances delirium, nightmares, and hallucinations can occur, though these are uncommon [181]. The most common side effects appear to be amnesia and parasomnias (sleep walking/eating/driving) and in rare occasions attempted para-suicide [180, 182, 183]. Zolpidem is also associated with increased major injury due to unintentional falls and vehicle incidents [184].

\section{Neuropharmacology and Neurocircuitry of Adverse Effects}

\section{Modulation of GABAARs}

Studies have looked into possible neuroadaptive mechanisms that may account for tolerance and withdrawal to BZs and zolpidem, focusing mainly on changes in $\mathrm{GABA}_{\mathrm{A}} \mathrm{R}$ subunits in in-vitro and in-vivo assays. Most studies investigate changes after long-term BZ treatment, but fewer have looked into the changes that may occur after long-term zolpidem treatment $[92,116,185]$. A main finding is that $\alpha 1$ subunit mRNA levels are decreased after chronic use and withdrawal from zolpidem and BZs in the cortex [106, $107,109,114]$. Zolpidem and diazepam causes decreases in $\alpha 1$ subunit mRNA levels in the prefrontal cortex [105]. Also it appears that some studies find decreases in subunits that make up $\alpha 1-\mathrm{GABA}_{\mathrm{A}} \mathrm{Rs}$ ( $\beta 2$ and $\gamma 2$ subunits) and increase subunits the $\alpha 4$ subunit [186]. This is in contrast to a study of recombinant $\alpha 1 \beta 2 \gamma 2 \mathrm{~S} \mathrm{GABA}_{\mathrm{A}} \mathrm{Rs}$ in HEK293 cells, finding increases in $\alpha 1$ and $\gamma 2 \mathrm{~S}$ mRNA expression after a 96 hour withdrawal from zolpidem [187].

There are differences in the time frame these changes occur and also differences in if they occur during chronic treatment or during withdrawal [92]. In cerebellar granule cells it was found that long term diazepam treatment and withdrawal caused a reduction of $\alpha 1$ and both $\gamma 2$ mRNA [186, 188]. This same study and others found that long term diazepam causes an increase in the $\alpha 4$ subunit mRNA and that withdrawal from diazepam caused an increase in $\alpha 4$ subunit protein $[188,189]$. In the rat cortex after 7 or 14 days of diazepam treatment there were no significant differences in amount of $\alpha 1$ subunit mRNA, but there were increases in $\alpha 3, \alpha 4$, and $\alpha 5$ subunit mRNA, as well as an increase in the $\alpha 5$ subunit in the PFC $[105,111]$. There were no changes in the $\beta 2$ subunit but there was a decrease in $\gamma 2$ subunit mRNA [111]. Alterations in protein expression also occur after 
$\mathrm{BZ}$ and zolpidem treatment and may be concurrent or an effect of the decrease in mRNA expression.

Uncoupling is another mechanism by which BZs and zolpidem may induce tolerance and possibly withdrawal. Uncoupling is defined as a disassociation of the GABA and BZ binding interface. Chronic diazepam and zolpidem caused a decrease in the GABA potentiation by $[\mathrm{H}]$ flunitrazepam binding (a measure of uncoupling) in rat cortical membranes and HEK cells containing recombinant GABA ${ }_{A}$ Rs [190, 191]. Flurazepam also decreases GABA potentiation by [H]flunitrazepam binding [192]. Zolpidem treatment also increases [H]flunitrazepam binding sites and increases $\alpha 1$ and $\gamma 2$ subunit mRNA alongside inducing uncoupling [191, 193]. Another measure of uncoupling is the reduced ability of BZs to enhance GABA induced IPSPs [194, 195]. Chronic diazepam decreases potentiation by GABA, indicating reduced $G A B A_{A} R$ function [188]. It appears uncoupling does not involve decreasing GABA $\mathrm{A}_{\mathrm{A}}$. It has been hypothesized that uncoupling involves altering the expression of $\alpha$ subunits and not affecting the amount of $\mathrm{GABA}_{\mathrm{A}}$ Rs. Internalization has been thought to mediate this change in expression by increasing internalization of the $\alpha 1$ subunit and increasing surface expression of $\alpha 4$ after chronic BZ treatment [196-199].

BZs acting on the reward pathway may play a role in development of dependence. Many BZs can induce addiction after long periods of use and some case studies of zolpidem find patients experiencing positive effects like euphoria and increased energy. There is some conflicting evidence on the role of $\alpha 1-G A B A_{A} R$ in mediating reward. It has been found that $\alpha 1-\mathrm{GABA}_{\mathrm{A}} \mathrm{Rs}$ may mediate dependence and abuse through activating the VTA. BZs and zolpidem modulate the VTA through activation of $\alpha 1-G A B A_{A}$ Rs on local interneurons [168]. The local interneurons no longer inhibit dopaminergic neurons in the VTA, allowing for increased firing. Though it has been found that zolpidem does not cause reward enhancement and that reward enhancement was not abolished in $\alpha 1$ point mutation mice (mice with a H101R mutation) making their $\alpha 1-\mathrm{GABA}_{\mathrm{A}} \mathrm{R} B Z$ binding pocket non-functional [165]

\section{Glutamate Receptors}

NMDA/AMPA. While changes in $\mathrm{GABA}_{\mathrm{A}} \mathrm{Rs}$ likely play a major role in mediating tolerance and withdrawal to BZs and zolpidem, changes in other neurotransmitter systems are also likely involved. GABA $\mathrm{ARs}_{\mathrm{A}}$ are commonly located on excitatory neurons, inhibiting these neurons to maintain optimal excitatory neurotransmission. Prolonged GABA inhibition caused by repeated BZ or zolpidem treatments may produce compensatory increases in NMDARs, AMPARs, and metabotropic glutamate receptors (mGluRs). Increases in these glutamate receptors may influence the development of tolerance and withdrawal to zolpidem and BZs.

In support of compensatory increases, the NMDAR antagonist MK-801 has been shown to block tolerance and withdrawal to various BZs [126, 200, 201]. Chronic BZs 
have been found to increase in-vitro glutamate release and NMDA-stimulated cGMP efflux [127, 202]. Chronic use of BZs have been found to increase the mRNA and protein levels of NMDAR and AMPAR subunits in the cortex and hippocampus and also decrease AMPAR subunits in the amygdala. In rats it has been found that the mRNA and protein levels of GluN1 and GluN2B subunits in the dentate gyrus are increased in rats tolerant to diazepam 24 hours after last injection $[118,119]$. In the CA1 and CA2 regions of the hippocampus, there were decreased NMDA-mediated currents as well as decreased GluN2B subunit mRNA and protein levels in hippocampal slices [119, 203]. GluN1 and GluN2B subunit protein expression has been found to be increased in the rat cerebral cortex as well [117].

GluR1 subunit mRNA expression was increased in the frontal cortex and hippocampus but the GluN1 subunit mRNA expression was not changed in either region during withdrawal 96 hours after diazepam cessation nor while tolerant[104]. In hippocampal slices there are also increases in AMPAR function after flurazepam withdrawal and increases in GluR1 subunit protein expression [102]. Withdrawal from chronic s.c. administration of diazepam caused an increase in AMPARs (increase in $\left[{ }^{3} \mathrm{H}\right]$ Ro48-8487) in the hippocampus and thalamus [53, 201]. Diazepam withdrawal lead to a decrease in GluR1 and GluR2 subunit mRNA expression in the amygdala and a decrease in GluR1 subunit mRNA expression in the cortex [53, 117]. Most of the studies find that AMPAR induced changes in mRNA and protein expression occur during withdrawal. AMPAR may have an effect on tolerance as GluR1-/- mice show reduced tolerance to but increased withdrawal effects to flurazepam[121]. Based on the previous studies it appears that changes in NMDAR and AMPAR expression due to chronic BZ usage primarily occur in the hippocampus.

Metabotropic Glutamate Receptors. mGlu5 when activated causes intracellular calcium $\left(\mathrm{Ca}^{+}\right)$to be released allowing for the activation of multiple kinases. mGlu5 has been found to be increased after 7 days of zolpidem treatment $(30 \mathrm{mg} / \mathrm{kg})$ in mice along with downstream proteins like phospholipase $C \beta$ and calcium/calmodulin-dependent protein kinase Ii $\alpha$ [204]. mGlu5 is co-localized and co-expressed with the $\alpha 1-\mathrm{GABA}_{\mathrm{A}} \mathrm{R}$ subunit in the hippocampus [205].

\section{BDNF}

It has been found that brain-derived neurotrophic factor (BDNF) may play a role in BZ and zolpidem tolerance. Acute triazolam and zolpidem decrease BDNF protein levels in the hippocampus [206]. Acute zolpidem causes an increase in association of methyl-CpG binding protein 2 (MeCP2) with BDNF promoter IV and an increase in the association of phosphorylated cyclic adenosine monophosphate (cAMP) response element binding protein ( $\mathrm{pCREB}$ ) with BDNF promoter I, though long-term treatment did not affect any of these factors [206]. It is known that BDNF decreases mIPSC. BDNF decreases both the surface expression of $\mathrm{GABA}_{\mathrm{A}} \mathrm{Rs}$ and modulates of $\mathrm{mRNA}$ expression. $B D N F$ decreases $G_{A B A_{A}} R$ surface expression in general and specifically by the 
JAK/STAT pathway BDNF downregulates $\alpha 1-\mathrm{GABA}_{A}$ Rs by activation of inducible cAMP early repressor (ICER) which binds to pCREB to decrease mRNA expression $[207,208]$.

\section{Kinases}

The cAMP -dependent protein kinase (PKA) and $\mathrm{Ca} 2+/$ phospholipid-dependent protein kinase (PKC) modulate GABAergic functioning. Both kinases modulate mIPSCs in opposing ways in cortex, hippocampus and other brain regions. PKA decreases mIPSC amplitudes in pyramidal cells in the hippocampus whereas PKC increases mIPSC peak amplitudes in granules cells [209]. PKA activates ICER to regulate $\alpha 1$ subunit mRNA expression by preventing the binding of pCREB with the GABRA1 promoter leading to a decrease in $\alpha 1-G_{A B A} R_{A}$ [210]. Chronic zolpidem has been found to increase phosphorylated $\mathrm{PKC} \gamma$ in the limbic forebrain [211].

\section{Influence of GABA Transmission on Sleep, Sedation, Motor Inhibition, and Reinforcement: Neurocircuitry}

\section{Sleep/Sedation}

Most sedative-hypnotics are positive modulators of GABA $A_{A}$ Rs. Sleep is a naturally occurring state of unconsciousness accompanied by altered consciousness, reduced reactivity to external stimuli, and reduced voluntary movement, while sedation is an artificially induced state where the level of arousal is suppressed [212]. Both states are accompanied by decreases in voluntary movements allowing motor activity to be used as an index for both behaviors as seen in many studies evaluating BZ hypnotics and sedatives [213, 214]. Major regions have been identified that modulate circadian rhythms, REM, non-REM sleep, and sleep-homeostasis. The preoptic area appears promote sleep as the ventrolateral preoptic area (vLPO) and the median preoptic nucleus (MnPO) provide GABAergic inhibitory projections to the basal forebrain and brainstem arousal systems. The GABAergic projections to the arousal system inhibit the release of histamine, 5-HT, dopamine, acetylcholine, and orexin to various cortical regions. Most of these regions with the exception of the LC express $\alpha 1-, \alpha 2-$, and $\alpha 3-\mathrm{GABA}_{\mathrm{A}} \mathrm{Rs}$ and are subject to zolpidem and diazepam, inducing sedation or sleep (Figure 2-5) [215, 216]. While the circuitry of sleep has been well defined this is not the case with sedation though there is some evidence sedation works along the same sleep pathway [217].

There is overlap in the behavioral aspects of sleep and sedation and work done to disseminate how modulation of GABA ${ }_{A}$ Rs affect both. In $\alpha 1(\mathrm{H} 101 \mathrm{R})$ and $\alpha 3(\mathrm{H} 126 \mathrm{R})$ mice diazepam do not affect sleep latency or sleep duration compared to control mice $[74,218]$. When zolpidem is given to $\alpha 1(\mathrm{H} 101 \mathrm{R})$ at a hypnotic dose (same dose used for measures of sedation, $5-10 \mathrm{mg} / \mathrm{kg}$ ) non-REM sleep is no different than wild-type mice[219]. However diazepam's and zolpidem's sedative effect is missing in $\alpha 1$ (H101R) 


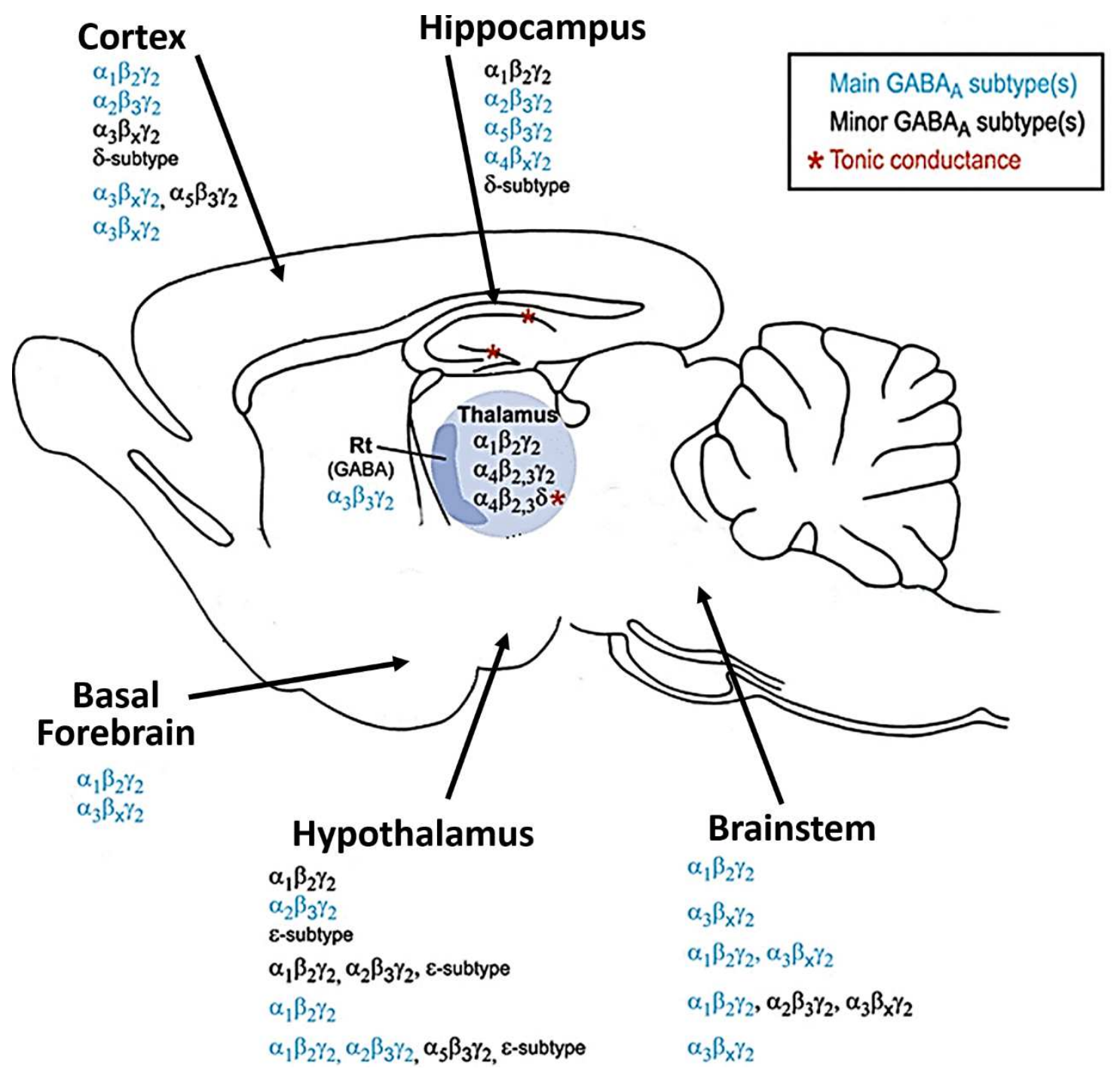

Figure 2-5. $\quad \mathrm{GABA}_{\mathrm{A}} \mathrm{Rs}$ modulated in the arousal system

$\mathrm{GABA}_{\mathrm{A}} \mathrm{Rs}$ are expressed in many regions that regulate arousal and sleep.

ACh, acetylcholine; ADO, adenosine; DA, dopamine; DpMe, deep mesencephalic reticular nucleus; DR, dorsal raphe nucleus; Glu, glutamate; Hcrt, hypocretins/orexins; His, histamine; LC, locus coeruleus; LDTg, laterodorsal tegmental nucleus; $\mathrm{MCH}$, melanin concentrating hormone; NA, noradrenaline; n.d., not determined; nRt, reticular nucleus of the thalamus; PeF, perifornical nucleus; $\mathrm{PH}$, posterior hypothalamus area; PPTg, pedunculopontine tegmental nucleus; SubC, subcoeruleus nucleus; TM, tuberomammillary nuclei; VLPAG, ventrolateral periacqueductal gray; VLPO, ventrolateral preoptic area; 5-HT, serotonin. Reprinted with permission. WinskySommerer, R., Role of GABAA receptors in the physiology and pharmacology of sleep. Eur J Neurosci, 2009. 29(9): p. 1779-94 [215]. 
$[98,214]$. Due to the effects found in point mutation mice it appears that modulation of $\alpha 1-G A B A_{A} R$ induces sedation while modulation of $\alpha 2-G A B A_{A} R$ induces sleep.

\section{Motor Inhibition}

Sedation in mice is usually measured as a decrease in motor activity. Movement is in general there is a balancing act between cortical excitation and inhibition from the basal ganglia. Excitation and inhibition of locomotor activity is controlled by the MLR and pedunculopontine nucleus (PPN) respectively within the mesopontine tegmentum. The MLR activates muscle tone through the locus caeruleus (LC), raphe nuclei and excitatory reticulospinal system and motion through CPGs. MLR activation is caused by activation of cholinergic and glutamatergic receptors. PPN decreases muscle tone hyperpolarizing the motoneurons and possibly suppressing CPGs possibly decreasing locomotion[220]. The basal ganglia specifically the substantia nigra pars reticulate (SNr) GABAergic neurons project to the MLR and PPN providing tonic inhibition and modulate locomotion and muscle tone. The basal ganglia receives innervation from the cerebral cortex and as stated before sends projections to the spinal cord through the mesopontine tegmentum.

The cortical-basal ganglia circuit is a major component of volitional movement. Glutamate and GABA control movement through direct and indirect circuits. This direct circuit is a loop in that the glutamatergic neocortical neurons projects to the striatum which also receives dopaminergic projections onto the D1 receptors both projections exciting the striatum. The striatum sends GABA to the internal pallidum inhibiting its GABAergic neurons which would send GABA to the thalamus, since the thalamus is no longer inhibited by the internal pallidum it then sends glutamate back to the neocortex thus initiating movement. Inhibition of movement occurs when the D2 receptors on the striatum of activated by dopamine leading to GABA release from the striatum onto the internal and external pallidum. The external pallidum would send inhibitory projections onto the subthalamic nucleus but due to the inhibition from the striatum does not and this allows the subthalamic nucleus to send glutamatergic projections to the internal pallidum causing a net excitation. With the internal pallidum activated it sends GABA to the thalamus restricting glutamatergic release onto the neocortex [221].

\section{Project Purpose}

The purpose of this project was to examine tolerance, cross-tolerance, withdrawal, and molecular alterations in zolpidem and diazepam-treated C57/BL6J mice after different treatment durations. Elucidating the mechanisms behind zolpidem tolerance is necessary due to the ongoing use and development of subunit specific GABA ${ }_{A}$ RAMs and the overall understanding of $\mathrm{GABA}_{\mathrm{A}} \mathrm{R}$ mechanisms. We hypothesized that 3 days of repeated zolpidem or diazepam would result in no behavioral and molecular alterations. We hypothesized that 7 days of zolpidem and diazepam would result in tolerance, crosstolerance, withdrawal, and alterations in GABAergic and glutamatergic mRNA and 
protein levels. We hypothesized that 30 days of zolpidem and diazepam would result in tolerance and cross-tolerance to zolpidem's effects and alter GABAergic mRNA levels. 


\section{CHAPTER 3. EXPERIMENTAL METHODS*}

The purpose of Study 1 was to determine the effects of 3 days of zolpidem and diazepam on the sedative effects of zolpidem treatment and mRNA levels of GABA $R$ subunits and $\mathrm{GABA}_{A} \mathrm{R}$ associated proteins. The purpose of Study 2 was to determine the effects of 7 days of zolpidem and diazepam on the sedative effects of zolpidem treatment, spontaneous withdrawal, flumazenil-induced withdrawal, and mRNA levels of $\mathrm{GABA}_{A} \mathrm{R}$ subunits, $\mathrm{GABA}_{\mathrm{A}} \mathrm{R}$ associated proteins, AMPAR subunits, and NMDA subunits. Study 2 also examined the effect of treatments on total and cell surface expression of $\mathrm{GABA}_{\mathrm{A}} \mathrm{R}$ receptor subunits proteins. The purpose of Study 3 was to determine the effects of 30 days of chronic zolpidem and diazepam on the sedative effects of zolpidem treatment and mRNA levels of $\mathrm{GABA}_{\mathrm{A}} \mathrm{R}$ subunits and $\mathrm{GABA}_{\mathrm{A}} \mathrm{R}$ associated proteins.

Studies 1-3 examined sedative tolerance by measuring the locomotor activity of mice during open field testing. Locomotor activity was defined as the horizontal distance traveled (in $\mathrm{cm}$ ) during a 60-min open field test. In rodents, decreases in open field locomotor activity is a standard behavioral test for assessing the sedative effects of drugs $[66,69,222-226]$. Tolerance to drug sedation is commonly observed as the diminished effectiveness to reduce locomotor activity $[25,105]$.

Withdrawal-like symptoms were measured by locomotor activity during open field testing and anxiety-like behavior in the elevated plus maze (EPM). In rodents and humans, abrupt discontinuation of a sedative can cause hyperactivity and anxiety-like effects [53, 121, 227]. Hyperactivity was characterized as increased distance traveled in the open field. The Open-field anxiety-like behaviors were identified by the decreases in the percent of time and entries into the center zone and a decrease in frequency with which the mice stood on their hind legs in the maze (frequency of vertical activity or rearing) [17]. Anxiety-like behaviors in the EPM were identified as decreases in the percent of entries or time in the open arms [228-231].Tolerance and withdrawal from BZs and zolpidem are also associated with alterations in mRNA expression. Messenger RNA was measured by real-time quantitative reverse transcription polymerase chain reaction (qRT-PCR). We examined mRNA expression in 4 brain regions: cortex, hippocampus, amygdala, and PFC. The cortex and PFC have roles in mediating locomotor activity and would possibly be affected by repeated diazepam and zolpidem [87]. It is thought that the hippocampus and amygdala have roles in mediating withdrawal induced anxiety and possibly tolerance from BZs [63, 232, 233].The cortex and prefrontal cortex have been found to have changes in mRNA expression due to BZs and zolpidem [107, 109, 111, $113,115,117,234]$. Other studies have found changes in mRNA expression in the hippocampus as well $[102,104,118,119,202,203]$. Tolerance and withdrawal from BZs

\footnotetext{
* Modified with permission. Wright, B.T., C.F. Gluszek, and S.A. Heldt, The effects of repeated zolpidem treatment on tolerance, withdrawal-like symptoms, and GABAA receptor $m R N A$ s profile expression in mice: comparison with diazepam. Psychopharmacology (Berl), 2014. 231(15): p. 2967-79 [16].
} 
and zolpidem can also alter total and surface expression of proteins in the cortex and hippocampus as measured by western blot [107, 117].

\section{Materials}

\section{Mice}

Male C57BL/6J mice about 3-6 months old and weighing about 18-30 g (Jackson Laboratories, Maine) were used in these studies. Mice were housed in standard group cages $(\mathrm{n}=5)$, allowed full access to food and water, and maintained on a 12 hour light/dark cycle (light on from 7:00 a.m.). Once in the vivarium, mice were allowed to acclimate there for at least a week before being used in experiments. Experiments were performed during the light cycle and were approved by the UTHSC Institutional Review Board. All experiments followed the principles and standards of animal care outlined by the NIH publication "Guide for the Care and Use of Laboratory Animals" [235].

In Study 1, two cohorts of mice were used for the experiments. Cohort 1 included mice treated with vehicle $(n=11)$, diazepam $(n=11)$, and zolpidem $(n=12)$. Cohort 2 of Study 1 included mice treated with vehicle $(\mathrm{n}=9)$, diazepam $(\mathrm{n}=10)$, and zolpidem $(\mathrm{n}=$ 9). In Study 2, five cohorts of mice were used for experiments. Cohort 1 included vehicle $(n=13)$, diazepam $(n=13)$, and zolpidem $(n=13)$ treated mice. Cohort 2 of Study 2 included mice treated with vehicle $(n=9)$, diazepam $(n=9)$, and zolpidem $(n=8)$ mice. Cohort 3 of Study 2 included mice treated with vehicle $(n=4)$, diazepam $(n=4)$, and zolpidem $(n=5)$ mice. Cohort 4 of Study 2 included mice treated with vehicle $(n=6)$, diazepam $(n=8)$, and zolpidem $(n=9)$ mice. Cohort 5 of Study 2 included mice treated with vehicle $(n=6)$, diazepam $(n=6)$, and zolpidem $(n=6)$ mice. In Study 3, two cohorts of mice were used. Cohort 1 was composed of vehicle $(n=6)$, diazepam $(n=6)$, and zolpidem $(\mathrm{n}=7)$ treated mice and Cohort 2 included mice treated with vehicle $(\mathrm{n}=$ $12)$, diazepam $(\mathrm{n}=10)$, and zolpidem $(\mathrm{n}=19)$ mice.

\section{Drugs and Antibodies}

Diazepam, zolpidem (catalog numbers D0899 and Z-103, respectively; Sigma, St Louis, MO) and flumazenil (catalog number 1328; Tocris, Bristol, UK) were suspended in a vehicle which was composed of saline with $0.05 \%$ Tween-80. Animal weights were recorded before all drug injections. Daily injections and behavioral testing were conducted in distinct environments to prevent context-specific tolerance [236]. Drugs were administered intraperitoneally (i.p.) at a volume of $10 \mathrm{ml} / \mathrm{kg}$. In Studies 1-3 mice were treated twice a day (8:00 am and 5:00 pm) with $5 \mathrm{mg} / \mathrm{kg}$ of zolpidem, $10 \mathrm{mg} / \mathrm{kg}$ of diazepam, or vehicle. Treatment doses were based on previous experiments in rats and mice designed to study the development of sedative tolerance to diazepam or zolpidem $[25,28,112,123,128,129]$. The test dose of zolpidem was selected from dose-response curves indicating the $50 \%$ effective dose (ED50) for the locomotor-impairing effects of zolpidem in rats and mice ranging from 1.0 to $2.5 \mathrm{mg} / \mathrm{kg}[72,224]$. The test dose of the 
$\mathrm{GABA}_{\mathrm{A}} \mathrm{R}$ antagonist flumazenil was used based on prior experiments showing flumazenil, at a dose of $10 \mathrm{mg} / \mathrm{kg}$, induces mild withdrawal symptoms like increased locomotor activity and anxiety-like behaviors in mice previously given chronic BZ treatments $[38,121,230,237]$.

Primary $\mathrm{GABA}_{\mathrm{A}} \mathrm{R}$ subunit antibodies used for cross-linking/western blot analyses were as follows: $\alpha 1$ subunit (1:1000; Millipore, Billerica, MA, USA), $\alpha 2$ subunit $(1: 1000$; Alomone Labs, Jerusalem, Israel), $\alpha 3$ subunit (1:500; Abcam, Cambridge, UK), and $\gamma 2$ subunit (1:1000; Affinity Bioreagents, Waltham, MA). The primary NMDAR subunits antibodies used were NR1 subunit (1:1000; Abcam, Cambridge, UK) and NR2A subunit (1:400; Millipore, Billerica, MA). Peroxidase labeled anti-rabbit IgG (1:5000) and peroxidase labeled anti-mouse IgG (1:5000) were used as secondary antibodies (both from Vector Laboratories, Burlingame, CA). An antibody for glyceraldehyde 3phosphate dehydrogenase (GAPDH, 1:3000; Fitzgerald, Acton, MA) was used as a control protein and to normalize results. The GAPDH was a monoclonal mouse antibody and the other primary antibodies were polyclonal rabbit antibodies.

\section{Justification for Why We Selected 3, 7, and 30 Days as Our Experimental Time Points}

Most drug studies investigate the behavioral and molecular changes that occur after different treatment durations of drugs. Most studies that have looked at zolpidem tolerance have examined development after 7-14 days, however there are few that look at the effects of short-term or long-term zolpidem treatment. Studies investigating BZ tolerance examine tolerance after 1-3 days (short-term), 7-14 days, and $>28$ days (longterm) $[122,127,130,131,151]$.

\section{Justification for Two Injections for Methodology}

For this project we decided to give mice two injections to better mimic the high dose levels that can occur when humans abuse zolpidem by self-administering multiple high doses, sometimes throughout the day [139, 147, 148, 174]. In addition, since mice

metabolize zolpidem faster than humans, two doses increase the probability of mimicking high circulating concentrations that occur in humans abusing zolpidem $[31,33,37,39$, $131,179]$. The $50 \%$ effective dose (ED50) for zolpidem in rodents needed to impair locomotor activity is $1.0-2.5 \mathrm{mg} / \mathrm{kg}$ and the ED50 for diazepam is $1.21 \mathrm{mg} / \mathrm{kg}[72,224$, 238]. The doses are 10x (zolpidem) or 20x (diazepam) greater than needed to induce sedation in mice. Likewise, humans who abuse zolpidem go beyond the clinical dose (5$10 \mathrm{mg}$ ) and can use 10-20 times the clinical dose (100-1000 $\mathrm{mg})$, defined as a supratherapeutic dose. 


\section{Methods}

Figure 3-1 is a simple flow-chart of the experimental designs of Studies 1-3. In all studies, mice received treatment injections of vehicle, diazepam, and zolpidem on consecutive days (i.e. 3, 7, or 30). All behavioral tests and molecular analyses were started 16-20 hours after the last treatment injection, unless otherwise stated. In Study 1, two cohorts of mice received vehicle, diazepam, or zolpidem for 3 days. Both cohorts were tested for sedative tolerance measured by locomotor activity in the open field. Immediately after testing Cohort 2 was given one injection of their assigned drug, then sacrificed 16-20 hours later for analysis of mRNA expression. The mRNA expression of $\mathrm{GABA}_{\mathrm{A}} \mathrm{R} \alpha 1-5, \beta 1-3, \gamma 1-2$ and $\delta$ subunits, GABA clustering protein gephyrin, and GABA transporter 1 (GAT-1) were measured by qRT-PCR.

In Study 2, five cohorts of mice received vehicle, diazepam, or zolpidem for 7 days. Cohort 1 was tested for sedative tolerance measured by locomotor activity in the open field. Cohort 2 was tested for spontaneous withdrawal measured by locomotor activity and anxiety-like behaviors measured in the open field. Cohort 3 was tested for flumazenil-induced withdrawal measured by anxiety-like measures in the EPM and open field as well as locomotor activity in the open field. Cohort 4 was used for analysis of mRNA expression of $\mathrm{GABA}_{\mathrm{A}} \mathrm{R} \alpha 1-5, \beta 1-3, \gamma 1-2$ and $\delta$ subunits, gephyrin, GAT-1, NMDA subunits (GluN2A, GluN2B, and GluN1), and AMPA subunits (GluR1 and GluR2) measured by qRT-PCR. Cohort 5 was used to analyze total protein expression of GABA $_{A} R$ subunits $\alpha 1, \alpha 2, \alpha 3$, and $\gamma 2$, and the surface expression of GABA $A_{A}$ subunit $\alpha 1$. Protein expression was measured by western blot in the cortex and hippocampus.

In Study 3, two cohorts of mice received vehicle, diazepam, or zolpidem for 30 days. Both cohorts were tested for sedative tolerance measured by locomotor activity in the open field. Immediately after testing Cohort 2 was given their assigned drug regimen, then sacrificed 16-20 hours later for analysis of mRNA expression of GABA $\mathrm{R} \alpha 1-5$, $\beta 1$ $3, \gamma 1-2$ and $\delta$ subunits, gephyrin, and GAT-1 measured by qRT-PCR. A separate cohort of mice were for injected for 1 day (acute), to measure the effect of acute diazepam, zolpidem, and vehicle on mRNA expression measured by qRT-PCR.

\section{Tolerance and Spontaneous Withdrawal: Open Field}

Tolerance and spontaneous withdrawal were measured by examining locomotor activity during a 60-min open field test session [226]. Mice were given either $2 \mathrm{mg} / \mathrm{kg}$ of zolpidem (tolerance) or vehicle (spontaneous withdrawal) and placed back in the home cage for 2 min prior to testing. A 2-min latency between test drug injection and testing onset has been successfully used to identify tolerance to the locomotor-impairing effect of the BZ midazolam, which, like zolpidem, is relatively short acting and rapidly eliminated [239, 240]. After 2 min, mice were individually placed in open field chambers (44.45 cm long, $45.09 \mathrm{~cm}$ wide, and $29.21 \mathrm{~cm}$ high) constructed from a clear polycarbonate and equipped with four 24-beam infrared arrays across the base of each chamber wall (MED Associates, Model ENV-520). All measures in the open field were 


\section{Study 1}

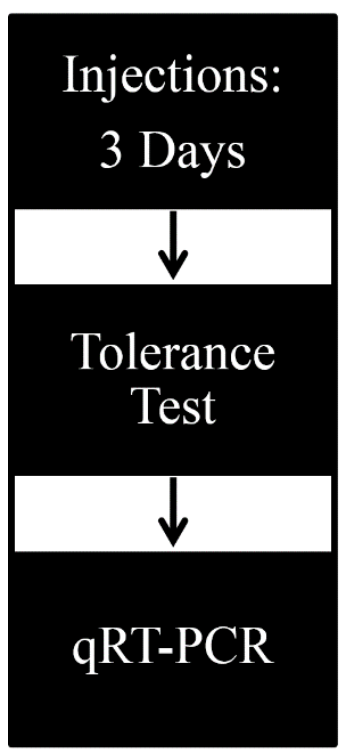

Study 2

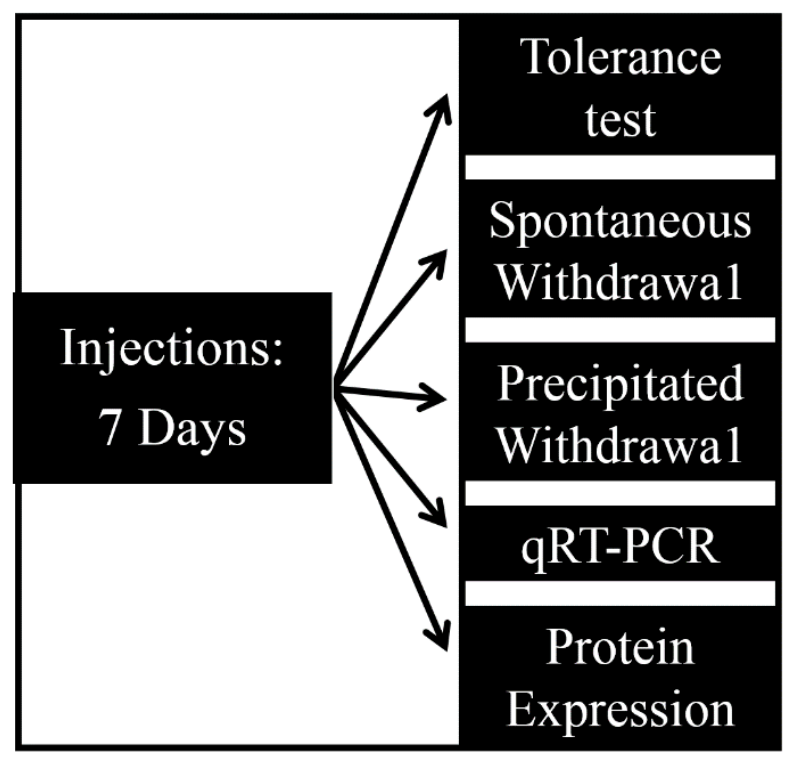

Study 3

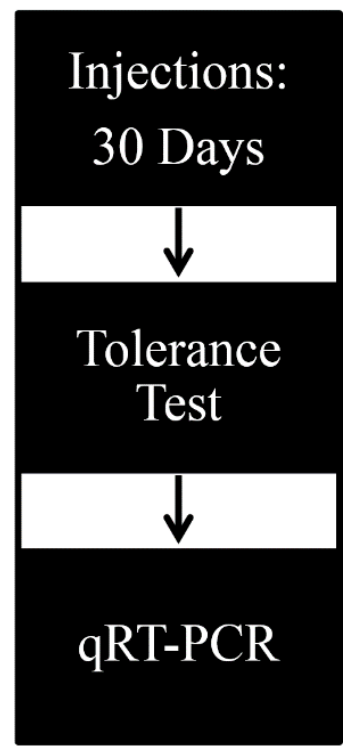

Figure 3-1. Overview flow chart of experimental studies

The studies were conducted to evaluate the effect of treatment durations on locomotor activity, mRNA levels, and protein expression. The mice used in these studies were male C57BL/6Js. In Study 1, two cohorts of mice were used, both were used for tolerance and cohort 2 was used for qRT-PCR. In Study 2, five cohorts of mice were used, each cohort was used in only one experiment. In Study 3, two cohorts of mice were used, both were used for tolerance and cohort 2 was used for qRT-PCR 
automatically recorded and collected by the MED Associates' Activity Monitor Data Analysis software installed on an IBM-based computer. For tolerance experiments, the horizontal distance (in $\mathrm{cm}$ ) was recorded and used as an index of motor sedation. For withdrawal experiments, horizontal distance, vertical counts (frequency of rearing), time spent in the center, and number of center entries were recorded and used as an indices of withdrawal. The central zone was defined as the compartment of the floor centrally located $8 \mathrm{~cm}$ from the perimeter of the chamber walls. Testing was conducted under standard test room lighting (100 lux), and each animal was tested once.

\section{Flumazenil-Induced Withdrawal: EPM and Open Field}

Cohort 3 was used to measure flumazenil-induced withdrawal. After a 7 day treatment with diazepam, zolpidem, or vehicle, mice were tested for flumazenil-induced withdrawal on two consecutive days. On Day 1 , mice were given a $10 \mathrm{mg} / \mathrm{kg}$ injection of flumazenil 20 min before a 5 min EPM test session. After EPM testing, mice were immediately placed in the open field for a 60 -min test session. On Day 2 mice were given a $10 \mathrm{mg} / \mathrm{kg}$ injection of flumazenil $20 \mathrm{~min}$ before a 60 -min open field test session. Mice were then individually placed in open field chambers.

The EPM was constructed of wood, painted white, and consisted of 4 arms that were $30.48 \mathrm{~cm}$ long and $6.99 \mathrm{~cm}$ wide and elevated by a wooden base to a height of $76.20 \mathrm{~cm}$. Two of the EPM arms were without walls and 2 arms were enclosed by $17.78 \mathrm{~cm}$ high walls. Animal movements were recorded overhead with the use of a camera (SENTECH). Recordings were analyzed with video-tracking software (ANYmaze $($, Stoelting CO, USA). The anxiety-like behaviors tracked in the EPM were percent time spent in the open arms and the number of entries into the open arms. The testing in the open field was conducted as stated above in Tolerance and Withdrawal: Open Field. For flumazenil-induced withdrawal the dependent variables were the same as for spontaneous withdrawal.

\section{qRT-PCR}

About a day after their last treatment injection, mice were sacrificed by cervical dislocation. Immediately afterwards, whole mouse brains were rapidly isolated and immediately frozen on crushed dry ice. Tissue isolation was performed by sectioning brains (300- $\mu$ mthick) on a Leica cryostat, followed by dissection of the PFC, cortex, amygdala, and the hippocampus using a stereomicroscope and a scalpel blade under RNAse-free conditions. After the collection, samples were stored in buffer RLT Lysis buffer (QUIAGEN) at $-20{ }^{\circ} \mathrm{C}$.

Samples were then homogenized by sonication and RNA was isolated with the RNeasy protect mini kit (QIAGEN). RNA concentration and purity were determined using a microplate reader in conjunction with a Take $3^{\mathrm{TM}}$ Micro-Volume plate (BioTek Instruments, Inc., Winooski, VT). The ratio of absorbance at 260 and $280 \mathrm{~nm}$ of samples 
were commonly in the range of 1.8-2.2. Samples with A260/A280 of less than 1.8 were considered to be contaminated by protein and excluded from further analyses. RNA integrity was determined using the Agilent Bioanalyzer 2100 (Agilent Technologies, UK) to generate an electropherogram and an RNA integrity number (RIN). RIN scores ranged between 5.3 and 9.4 .

RNA was transcribed to cDNA using Transcriptor first-strand cDNA synthesis kit (Roche Diagnostics, Indianapolis, IN, USA) following the manufacturer's instructions. One reaction consisted of $2 \mu \mathrm{L}$ Anchored oligo(dT) primer $(2.5 \mu \mathrm{M}), 4 \mu \mathrm{L}$ random hexamer primer $(60 \mu \mathrm{M}), 8 \mu \mathrm{L}$ reaction buffer $5 \times(8 \mathrm{mM}), 1 \mu \mathrm{L}$ protector TNase inhibitor (20 $\mathrm{U}), 4 \mu \mathrm{L}$ deoxynucleotide mix $(1 \mathrm{mM})$, and $1 \mu \mathrm{L}$ reverse transcriptase $(10 \mathrm{U})$. Varying volumes of RNA (amounting to $100 \mathrm{ng}$ ) and PCR-grade water were added for a $20-\mu \mathrm{L}$ reaction mixture. Complementary DNA (cDNA) was transcribed using an Eppendorf Mastercycler thermal cycler (Eppendorf) gradient PCR machine. Reverse transcription incubation conditions were $25^{\circ} \mathrm{C}$ for $10 \mathrm{~min}, 55^{\circ} \mathrm{C}$ for $30 \mathrm{~min}$, and $85{ }^{\circ} \mathrm{C}$ for $5 \mathrm{~min}$.

The relative expression levels of $\alpha 1-5, \beta 1-3, \gamma 1-2$ and $\delta$ subunits, gephyrin, and GAT-1 were measured in Studies 1-3. In Study 2 the relative expression levels of GluN2A, GluN2B, GluN1, GluR1 and GluR2 were also measured. Measurements for mice given acute administration were limited to $\alpha 1-3, \alpha 5, \beta 2, \gamma 2$, and GAT-1. Primer and probe combinations were designed using ProbeFinder software (version 2.45; Roche Diagnostics, Indianapolis, IN, USA). The oligonucleotide sequences used for the primers (Integrated DNA Technologies) and gene transcript IDs are shown in Table 3-1[16].

To determine PCR efficiencies, we generated standard curves for each target and reference genes ( $\beta$-tubulin, $\beta$-actin, cyclin $\mathrm{D}$, hypoxanthine phosphoribosyltransferase 1 , ribosomal protein S19, and TATA-binding protein) by plotting the threshold value $(\mathrm{Ct})$ versus the $\log$ of the amount of serial dilutions of cDNA $(2 \mu \mathrm{g}-0.2 \mathrm{ng})$. Separate standard curves were generated for each brain region analyzed. The amplification efficiencies of target and reference genes were within an acceptable slope range of -3.6 to -3.1 , corresponding to efficiencies close to $100 \%$. TATA-binding protein was used as the endogenous reference gene and amplified in parallel with target genes. This reference gene showed stable expression across samples and between brain regions. PCR efficiencies between the target and reference genes were relatively equal. Real-time PCR was performed using the TaqMan detection method and LightCycler ${ }^{\circledR} 480$ System (Roche Diagnostics, Indianapolis, IN, USA) [241]. Each reaction was performed in triplicate and consisted of $0.1 \mu \mathrm{L}$ of UPL probe $(10 \mu \mathrm{M}), 0.1 \mu \mathrm{L}$ of forward primer $(20$ $\mu \mathrm{M}), 0.1 \mu \mathrm{L}$ of reverse primer $(20 \mu \mathrm{M}), 5 \mu \mathrm{L}$ of LC $4802 \mathrm{X}$ master mix (Roche Diagnostics, Indianapolis, IN, USA), $2.7 \mu \mathrm{L}$ of PCR grade water, and $2 \mu \mathrm{L}$ of cDNA (10 ng). The PCR reaction consisted of initial incubations at $95^{\circ} \mathrm{C}$ for 5 min followed by 50 amplification cycles of $95^{\circ} \mathrm{C}$ for $10 \mathrm{~s}, 60^{\circ} \mathrm{C}$ for $30 \mathrm{~s}$, and $72{ }^{\circ} \mathrm{C}$ for $10 \mathrm{~s}$ 
Table 3-1. Gene transcripts and primer sequences

\begin{tabular}{|c|c|c|c|}
\hline Gene ID & Forward Primer Sequence & Reverse Primer Sequence & Ensembl Transcript ID \\
\hline Gabra 1 & 5'-CTT TTC TCC CGG GTC TGG-3' & 5'-TCT TCA TCA CGG GCT TGC-3' & ENSMUST00000169305 \\
\hline Gabra2 & 5'-ACA AAA AGA GGA TGG GCT TG-3' & 5'-TCA TGA CGG AGC CTT TCT CT-3' & ENSMUST00000000572 \\
\hline Gabra3 & 5'-CTT GGG AAG GCA AGA AGGTA-3' & 5'-GGA GCT GGT GTT TTC TT-3' & ENSMUST00000137517 \\
\hline Gabra4 & 5'-AAA GCC TCC CCC AGA AGT T-3' & 5'- CAT GTT CAA ATT GGC ATG TGT-3' & ENSMUST00000031121 \\
\hline Gabra5 & 5'-GAC GGA CTC TTG GAT GGC TA-3' & 5'-ACC TGC GTG ATT CGC TCT-3' & ENSMUST00000068456 \\
\hline Gabrb1 & 5'-CCC TCT GGA TGA GCA AAA CT-3' & 5'-AAT TCG ATG TCA TCC GTG GTA-3' & ENSMUST00000031122 \\
\hline Gabrb2 & 5'-CGA TGG CAC TGT CCT GTA TG-3' & 5'-ATA CCG CCT TAG GTC CAT CA-3' & ENSMUST00000169077 \\
\hline Gabrb3 & 5'-CGT GGG TGT CCT TCT GGA T-3' & 5'-ATG GTG AGC ACG GTG GTA AT-3' & ENSMUST00000085240 \\
\hline Gabrd & 5'-CGGAGCTGATGAACTTCAAAT-3' & 5'-ATGTAGACGCCCCGGTTC-3' & ENSMUSG00000029054 \\
\hline Gabrg1 & 5'-AGG CAG GAA GCT GAA AAA CA-3' & 5'-TTC ATG GGA ATG AGA GTG GA-3' & ENSMUST00000101143 \\
\hline Gabrg2 & 5'-ACA GAA AAT GAC GCT GTG GA-3' & 5'-CAT CTG ACT TTT GGC TTG TGA A-3' & ENSMUST00000070725 \\
\hline GEPHYRIN & 5'-TGA TCT TCA TGC TCA GAT CCA-3' & 5'-GCA AAT GTT GTT GGC AAG C-3' & ENSMUST00000052472 \\
\hline GAT-1 & 5'-GCC TGG TCA ATA CCA CCA AC-3' & 5'-CCA TCT GTC ATC TGG TGC AT-3' & ENSMUST00000032454 \\
\hline Grin2a & 5'-ATT CAA CCA GAG GGG CGT A-3' & 5'-TTC AAG ACA GCT GCG TCA TAG-3' & ENSMUSG00000059003 \\
\hline Grin $2 b$ & 5'-GTGAGAGGAAATCTCGGGGC-3' & 5'- GGATGCCGGGGATAGAAAGG-3' & ENSMUSG00000030209 \\
\hline Grin1 & 5'-GTCCTCCAAAGACACGAGCA-3' & 5'-AGCTCTCCCTATGACGGGAA-3' & ENSMUSG00000026959 \\
\hline Grial & 5'-AGGGATCGACATCCAGAGAG-3' & 5'-TGCACATTTCCTGTCAAACC-3' & ENSMUSG00000020524 \\
\hline Gria2 & 5'-CCAATGGGATAAGTTCGCATA-3' & 5'-GCACAGCTTGCAGTGTTGA-3' & ENSMUSG00000033981 \\
\hline
\end{tabular}

The gene transcripts and sequences used in the qRT-PCR experiments. Gabra1- $\alpha 1$ subunit, Gabra2- $\alpha 2$ subunit, Gabra3- $\alpha 3$ subunit, Gabra4- $\alpha 4$ subunit, Gabra5- $\alpha 5$ subunit, Gabrb1- $\beta 1$ subunit, Gabrb2- $\beta 2$ subunit, Gabrb3- $\beta 3$ subunit, Gabrd- $\delta$ subunit, Gabrg1- $\gamma 1$ subunit, Gabrg2- $\gamma 2$ subunit, Grin2a-GluN2A, Grin2b-GluN2B, Grin1-GluN1, Gria1GluR1, and Gria-GluR2. Modified with permission. Wright, B.T., C.F. Gluszek, and S.A. Heldt, The effects of repeated ZOLP treatment on tolerance, withdrawal-like symptoms, and GABAA receptor $m R N A$ s profile expression in mice: comparison with DZP. Psychopharmacology (Berl), 2014. 231(15): p. 2967-79 [16]. 


\section{Bissulfosuccinimidyl Suberate (BS3) Cross-Linking/Western Blot}

The water soluble protein BS3 reacts with cell-surface proteins to selectively form amine bonds on the cell surface. These cross-linked surface proteins can be isolated, visualized, and quantified by western blot analysis because they appear at a higher molecular weight compared to non-cross-linked intracellular protein [242].

About a day after mice were given their last treatment injection in Study 2, they were sacrificed by cervical dislocation. The whole mouse brains were rapidly isolated and placed on ice. BS3 cross-linking assays were performed following a protocol modified from Boudreau [242]. Tissue isolation was performed by sectioning brains (1mm thick) with an acrylic matrix (Brain Tree $\mathbb{C}$ ), followed by dissection of the left and right cortex and hippocampus with a scalpel blade. After dissection, right cortex and hippocampus tissue samples were placed in tubes containing artificial cerebrospinal fluid (ACSF) and stored on ice. Left cortex and hippocampus samples were placed in tubes containing a $52 \mathrm{mM} \mathrm{BS3/ACSF}$ solution and incubated for $30 \mathrm{~min}$ in a $4{ }^{0} \mathrm{C}$ refrigerator with gentle mixing. After BS3 incubation, the tissue was quenched in $100 \mathrm{mM}$ glycine solution and placed back into the $4^{\circ} \mathrm{C}$ refrigerator for a 10 min gentle mixing period. After quenching was completed, BS3 treated and non-treated tissue samples were spun down in for 2 min and the supernatant was discarded. Cortical and hippocampal BS3 treated and untreated tissue samples were placed in lysis buffer containing protease and phosphatase inhibitors (Complete ULTRA tablets, Roche Diagnostics, Indianapolis, IN, USA) and sonicated to lyse cells. Samples were again spun down and supernatant was collected. The protein concentration was quantified by the Pierce ${ }^{\mathrm{TM}} \mathrm{BCA}$ protein assay kit (ThermoFisher Scientific, Waltham, MA).

Equal amounts of protein $(20 \mathrm{mg})$ were separated by SDS-PAGE and then transferred to a nitrocellulose membrane (Bio-Rad). After transfer, the membrane was blocked with tris-buffered saline with $0.05 \%$ Tween 20 (TBST) containing 5\% milk for 1 $\mathrm{hr}$ at room temperature. After the membrane was blocked, it was incubated with primary antibody $\left(\alpha 1, \alpha 2, \alpha 3, \gamma 2\right.$, GluN1, GluN2B) diluted in TBST, overnight at $4^{0} \mathrm{C}$. The membrane was washed three times in TBST, and incubated with horseradish peroxidaseconjugated second antibody diluted in TBST at room temperature for $1 \mathrm{hr}$. After incubation, the membrane was washed three times in TBST. Proteins were visualized by an enhanced chemiluminescence detection system (Pierce ECL Western Blotting Substrate, Thermo Fisher Scientific GermanyLtd. \& Co.KG) and images were taken with a gel imaging system (Alpha Innotech,Thermo Fisher Scientific GermanyLtd. \& Co.KG). The GAPDH antibody (Fitzgerald) diluted in TBST was used as a control protein. The results of western blotting were analyzed by the AlphaView software system. All results were normalized with GAPDH. 


\section{Data Reduction and Statistical Analyses}

Tolerance, Spontaneous, and Flumazenil-Induced Withdrawal. In Studies 1-3, group differences in tolerance were assessed by analyses on distance traveled during each 10-min block using a two-way ANOVA with treatment (VEH, DZP, and ZOLP) as the between-subject factors and time (Blocks 1-6) as a within-subject factor. Follow-up analyses were done with one-way ANOVAs and Dunnett's $t$-tests where appropriate.

For spontaneous withdrawal, two-way ANOVAs were conducted on distance traveled in the center, periphery, and entire apparatus. These ANOVA were performed to assess whether distance traveled across the test session time (Blocks 1-6) influenced measures of open-field anxiety. Two-way ANOVAs were conducted on each of the following anxiogenic measures; frequency of rearing, number of center zone entries, and percent of center zone time during each 10-min block. The between-subject factor was treatment (VEH, DZP, and ZOLP) and the within-subject factor was time (Blocks 1-6). Follow-up analyses were done with one-way ANOVAs and Dunnett's $t$-tests where appropriate on each measure.

For flumazenil-induced withdrawal, analyses were conducted on percent time open arm time and entries into open arms EPM using a one-way ANOVA for each measure. Distance traveled, frequency of rearing, number of center zone entries, and percent of center zone time during each 10-min block on each day were analyzed using two-way ANOVAs for with treatment (VEH, DZP, and ZOLP) as between-subject factors and time (blocks 1-6) as a within-subject factor for each measure. Follow-up analyses were done with one-way ANOVAs and Dunnett's $t$-tests where appropriate for each open field measure.

mRNA and Protein Expression. Calculations of standard curves, CT values, and quantification of mRNA expression levels were performed by Gene Scanning software (version 1.5.0) provided with the LightCycler® 480 Instrument (Roche Applied Science). The comparative $\mathrm{CT}$ method $(\Delta \Delta \mathrm{CT}=\Delta \mathrm{CT}$ reference- $\Delta \mathrm{CT}$ target) was used to compare expression levels of mRNAs between the control (VEH) and treatment (ZOLP and DZP) groups. For ease of presentation, data in tables and figures are expressed as relative fold change by applying the 2- $\Delta \Delta \mathrm{CT}$ equation. Statistical comparisons were carried out for each analyzed region (cortex hippocampus, amygdala, and prefrontal cortex) and treatment protocol independently. Statistical analyses for mRNA and protein expression in the cortex and hippocampus were performed using one-way ANOVAs with Dunnett's $t$-tests for pairwise comparisons against a single control (VEH) to maintain a 0.05 error rate for each analysis. In vehicle-treated animals, the relative abundance of mRNA was as follows: cortex, $\alpha 1>\gamma 2>\beta 2>\alpha 3>\alpha 4>\beta 3>\delta>\alpha 5>\beta 1>\alpha 2>\gamma 1$; hippocampus, $\gamma 2>$ $\alpha 5>\beta 3>\alpha 1>\beta 2>\alpha 4>\beta 1>\alpha 2>\alpha 3>\delta>\gamma 1$; amygdala, $\gamma 2>\alpha 3>\beta 2>\alpha 1>\beta 3>\alpha 4$ $>\gamma 1>\beta 1>\alpha 5>\alpha 2>\delta$; and PFC, $\alpha 1>\alpha 3>\gamma 2>\beta 2>\beta 3>\alpha 2>\beta 1>\alpha 5>\delta>\alpha 4>\gamma 1$. 


\section{CHAPTER 4. RESULTS*}

\section{Study 1: The Effect of 3-Day Zolpidem and Diazepam Treatment on Behavioral Sedation and mRNA Expression}

\section{Tolerance to the Sedative Effects of Zolpidem}

The purpose of Study 1 was to examine if a 3-day treatment regimen of zolpidem or diazepam induces significant changes to the sedative effects of zolpidem, as measured by open field activity, and significant changes in mRNA expression, as measured by qRT-PCR. Alterations to the sedative effects were examined in Cohort 1 and 2 mice previously treated with vehicle, diazepam, or zolpidem (Figure 4-1). A two-way ANOVA found significant effects of time (Blocks), $F(5,290)=44.94, p<0.01$, treatment (VEH, DZP, ZOLP), $F(2,58)=4.08, p<0.05$, and a significant interaction of Time $\mathrm{x}$ Treatment, $F(10,290)=3.79, p<0.01$. The main effect of time indicated that activity between Blocks differ from each other, generally increasing over time. The main effect of treatment indicated significant differences between treatment groups in overall activity, independent of time. One-way ANOVAs performed at each level of time revealed significant differences at Blocks 2 and $3, F(2,58)=10.84, p<0.01$ and $F(2,58)$ $=6.20, p<0.01$, respectively. There were no differences at Blocks 1 and 4-6, $F \mathrm{~s}(2,58)<$ $6.20, p \mathrm{~s}>0.05$. Follow-up Dunnett's $t$-tests with VEH mice as controls showed the DZP mice displayed more activity at Blocks 2 and 3 than the VEH mice, $p$ s $<0.05$. These analyses indicated that after 3 days of diazepam, but not zolpidem, tolerance to zolpidem's sedative effects occur.

\section{Changes in mRNA Expression Measured by qRT-PCR}

Cohort 2 mice were used to examine changes in mRNA expression. One-way ANOVAs were used to compare group levels of each mRNA in the cortex, hippocampus, amygdala, and PFC. These ANOVAs revealed no significant differences in levels of any $\mathrm{GABA}_{\mathrm{A}} \mathrm{R}$ subunit mRNA or GABA $\mathrm{A}$-related mRNA in the cortex, $F \mathrm{~s}(2,24)<1.3, p \mathrm{~s}>$ 0.05 , amygdala, $F \mathrm{~s}(2,19)<0.94, p \mathrm{~s}>0.05$, or $\mathrm{PFC}, F_{\mathrm{s}}(2,25)<1.9, p \mathrm{~s}>0.05$ (Figure 4-2).

In the hippocampus, there was a significant treatment effect on the expression of $\alpha 5-\mathrm{GABA}_{\mathrm{A}} \mathrm{R}$ mRNA, $F(2,24)=3.45, p<0.05$. A Dunnett's $t$-test showed the ZOLP group had reduced $\alpha 5$-subunit expression compared to the VEH group, $p<0.05$. There were no significant treatment differences in the levels of other mRNA, $F s(2,24)<3.45$, $p s>0.05$ (Figure 4-3).

*Modified with permission. Wright, B.T., C.F. Gluszek, and S.A. Heldt, The effects of repeated zolpidem treatment on tolerance, withdrawal-like symptoms, and GABAA receptor $m R N A$ s profile expression in mice: comparison with diazepam. Psychopharmacology (Berl), 2014. 231(15): p. 2967-79 [16]. 


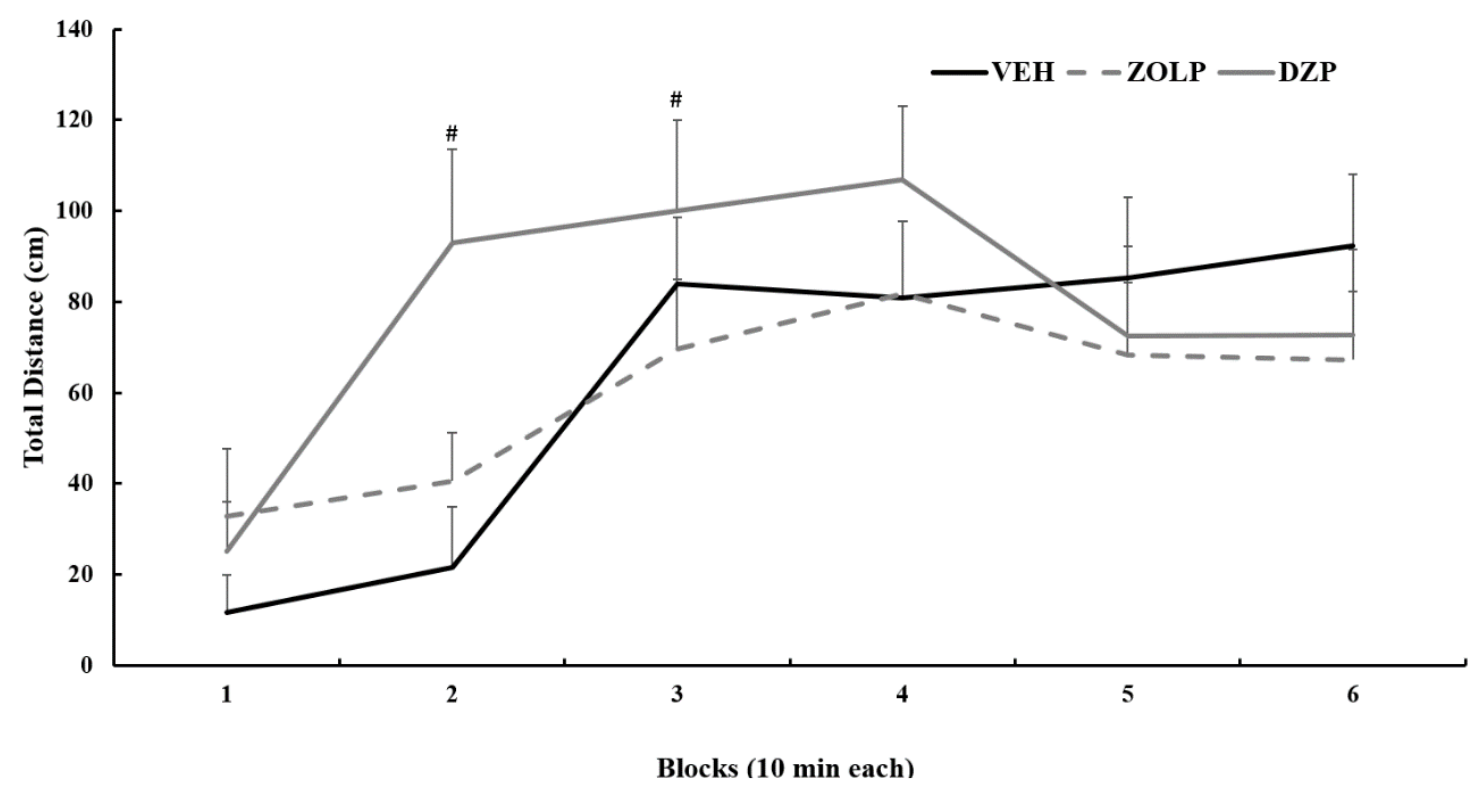

Figure 4-1. Decreased locomotor impairing effect of zolpidem after 3-day administration of zolpidem, diazepam, or vehicle

Distance traveled in open field after test administration of $2 \mathrm{mg} / \mathrm{kg}$ of ZOLP in mice previously given subchronic treatment of ZOLP, DZP, or VEH. Analyses of each 10-min block showed that differences between groups were most evident in the first half of the 60 -min test session. Data are presented as mean + sem. Number sign denotes VEH $<$ DZP groups $(p<0.05)$. 

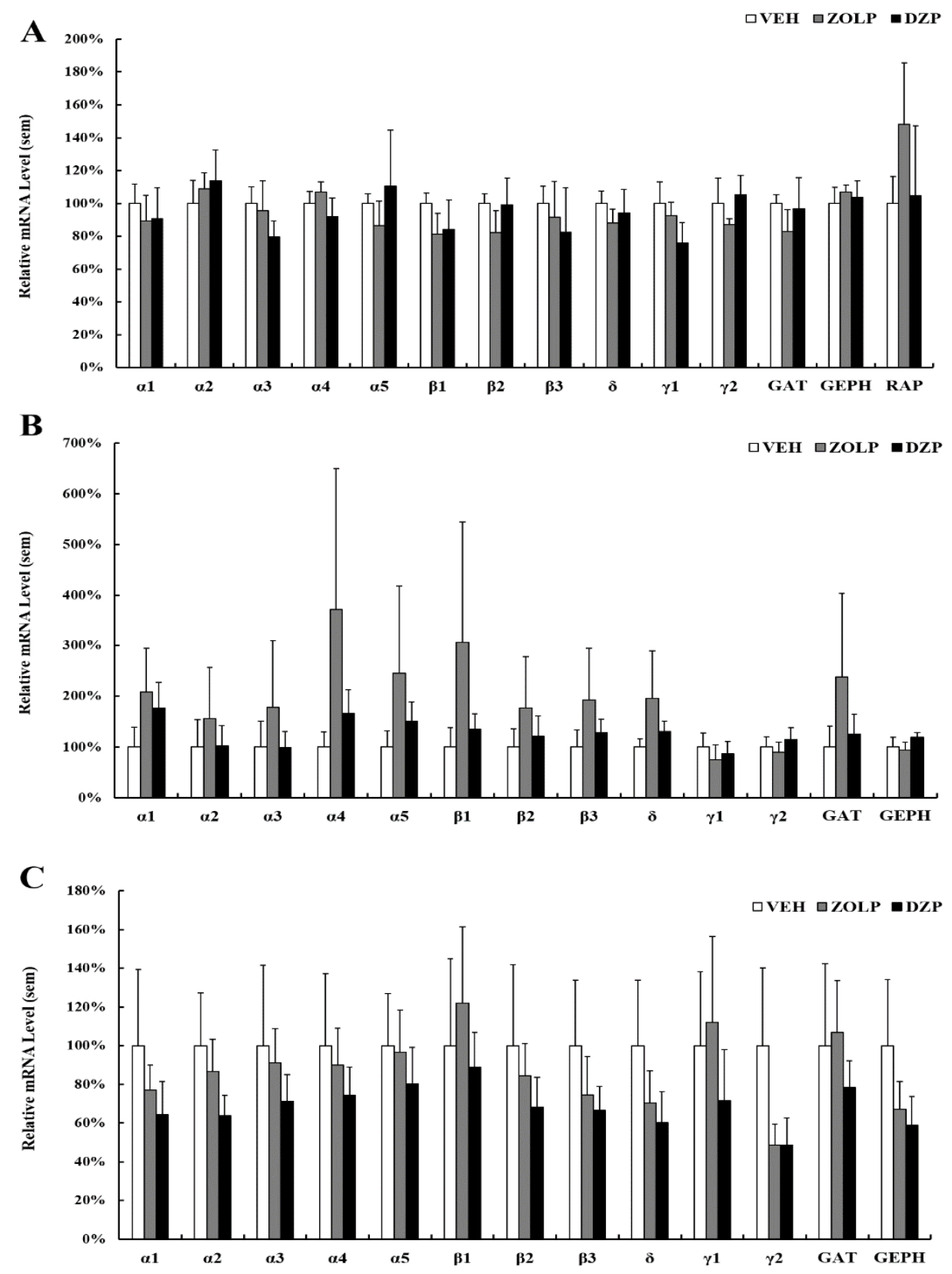

Figure 4-2. Relative levels of mRNA in the cortex, amygdala, and pfc following 3day administration of zolpidem, diazepam, or vehicle

Histograms comparing levels of transcripts are expressed as mean percentage of VEH control (100\%). Statistical analyses were performed by ANOVA with Dunnett's $t$-test for pairwise comparisons against a VEH for (A) cortex, (B) amygdala, and (C) pfc. Data are presented as mean + sem. There was no significance among these regions, $p>0.05$. 


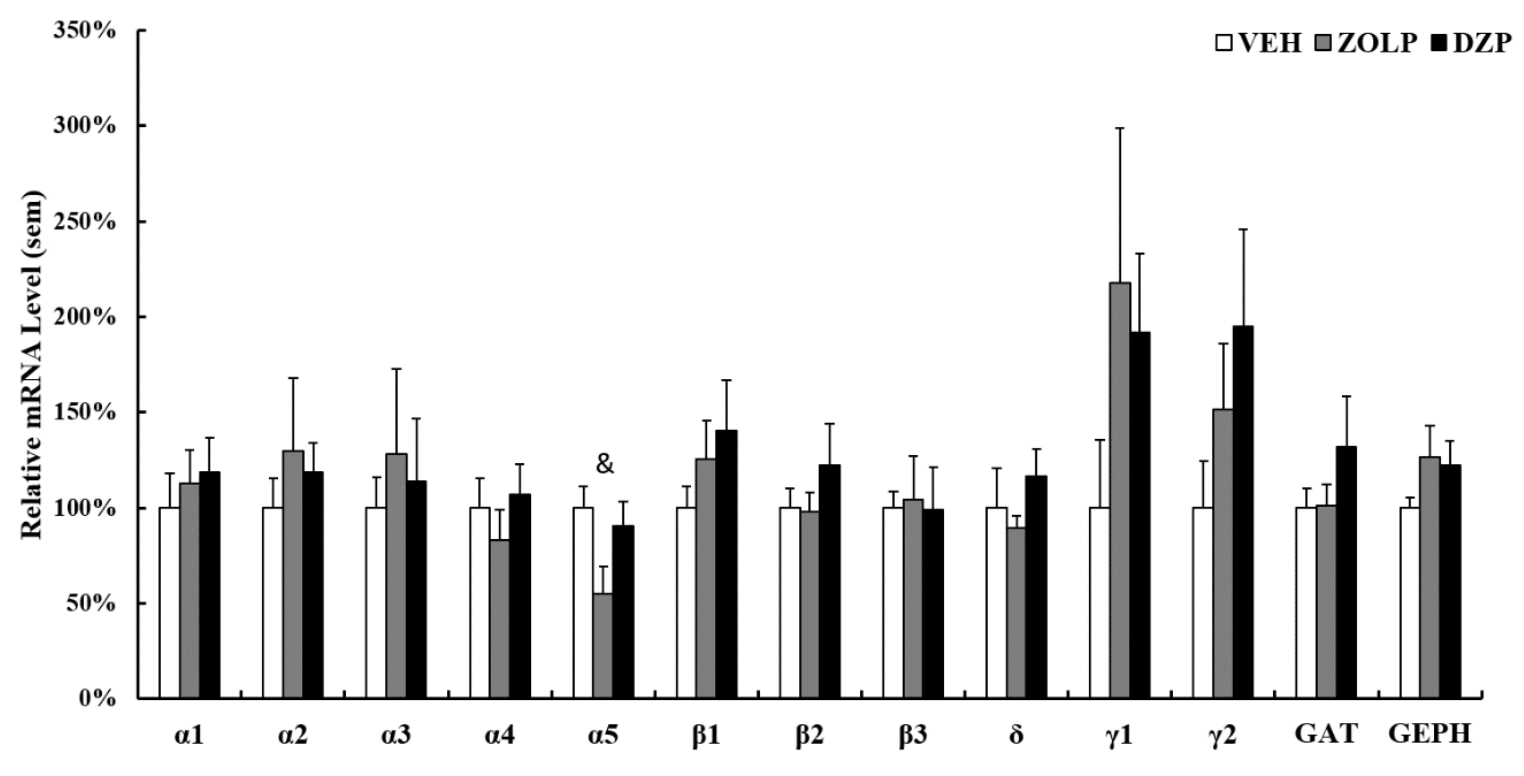

Figure 4-3. Relative levels of mRNA in the hippocampus following 3-day administration of zolpidem, diazepam, or vehicle

Histograms comparing levels of transcripts are expressed as mean percentage of VEH control $(100 \%)$. Statistical analyses were performed by ANOVA with Dunnett's $t$-test for pairwise comparisons against a VEH. Data are presented as mean + sem. Ampersand denotes VEH $>$ ZOLP treatments $(p<0.05$. $)$. 
In summary, results from Study 1 indicated that the sedative effects of zolpidem was significantly reduced in mice given diazepam for 3 days. The decreased sedative efficacy of zolpidem in mice given prior exposure to diazepam is indicative of crosstolerance. In contrast, the efficacy of zolpidem was unaffected in mice previously given zolpidem, suggesting no tolerance developed in these mice. There were no significant changes in the expression of $\mathrm{GABA}_{\mathrm{A}} \mathrm{R}$ subunit or associated mRNAs in the cortex, hippocampus, amygdala, or PFC after 3 days of diazepam. However, the expression of $\alpha 5-\mathrm{GABA}_{\mathrm{A}} \mathrm{R}$ mRNA was reduced in the hippocampus after 3 days of zolpidem treatment.

\section{Study 2: The Effect of 7-Day Zolpidem and Diazepam Treatment on Behavioral Sedation, Withdrawal, mRNA, and Protein Expression}

The purpose of Study 2 was to examine if a 7-day treatment regimen of zolpidem or diazepam causes 1) changes in the sedative effect of zolpidem, 2) spontaneous withdrawal after discontinuation of treatment, 3) flumazenil-induced withdrawal, 4) changes in mRNA expression measured by qRT-PCR, and/or 5) changes in total and cell surface expression of selective receptor subunits, as measured by western blotting.

\section{Tolerance to the Sedative Effects of Zolpidem}

Changes to the sedative effects of zolpidem were examined in Cohort 1 mice that were previously treated with vehicle, diazepam, or zolpidem. A two-way ANOVA yielded significant main effects of treatment, $F(2,46)=3.40, p<0.05$, time, $F(5,230)=$ $118.80, p<0.01$, and a reliable Treatment $\times$ Time interaction, $F(10,230)=8.00, p<$ 0.01 , signifying performance over time was differentially affected by drug treatment. To assess the interaction, one-way ANOVAs at each level of time were performed. These tests revealed group differences at Blocks 1, 2 and 3, $F(2,46)=8.16, p<0.01 ; F(2,46)=$ $16.34, p<0.01$; and $F(2,46)=6.61 p<0.01$, respectively. Follow-up Dunnett's $t$-tests revealed that the activity in ZOLP and DZP groups was significantly greater than in the VEH group at Blocks $1-3, p \mathrm{~s}<0.05$ (Figure 4-4). There were no group differences at Blocks 4-6, Fs $(2,36)<3.05, p s>0.05$.

\section{Spontaneous Withdrawal}

The presence of spontaneous withdrawal after discontinued zolpidem, diazepam, or vehicle treatments was assessed in Cohort 2. We looked at overall activity to determine if mice had the differences in the rate of habituation over time, which might confound our interpretation of results. A 2-way ANOVA found no significant main effect of time, $F(5,105)=0.01, p>0.05$, no main effect of treatment, $F(2,21)=0.02, p>0.05$, and no significant interaction of Time $\mathrm{x}$ Duration, $F(10,105)=0.11, p>0.01$. The analysis for rate of habituation show that all groups show a similar rate of habituation. 


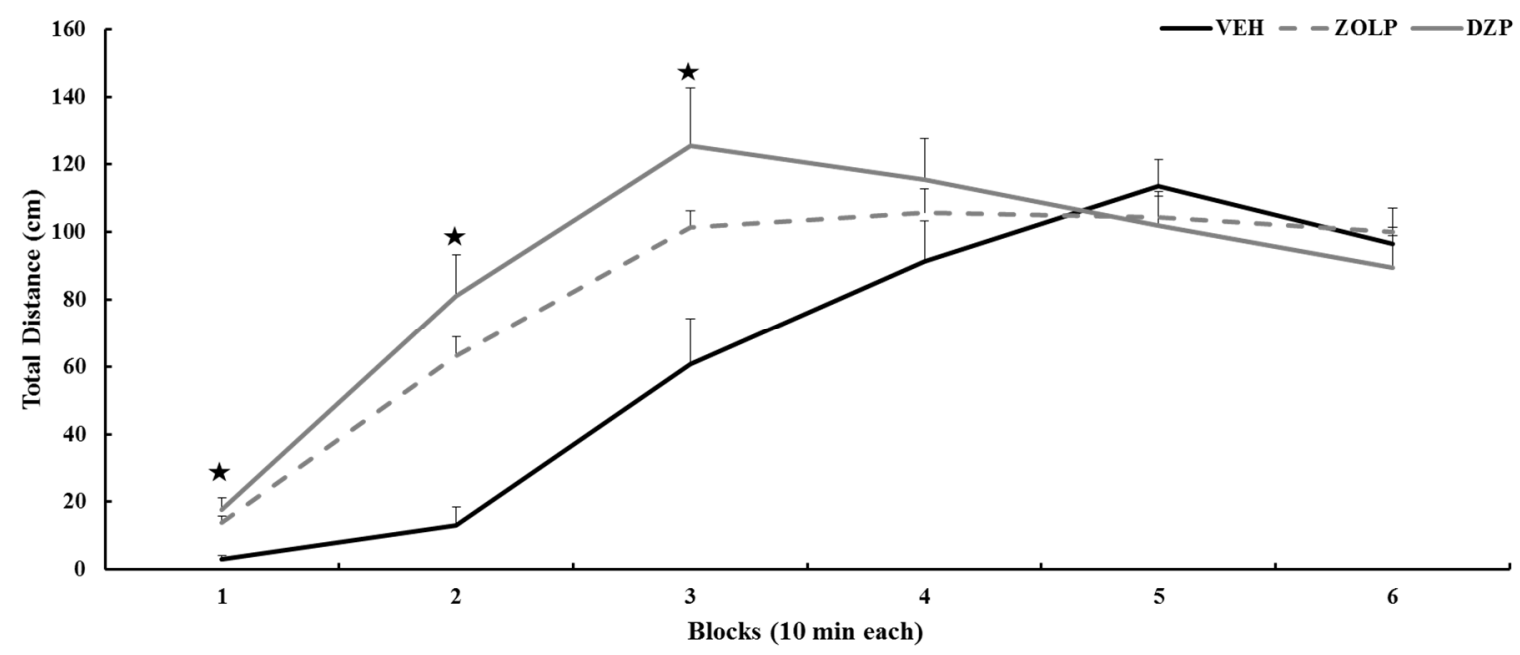

Figure 4-4. Decreased locomotor impairing effect of zolpidem after 7-day administration of zolpidem, diazepam, or vehicle

Distance traveled in open field after test administration of $2 \mathrm{mg} / \mathrm{kg}$ of ZOLP in mice previously given subchronic treatment of ZOLP, DZP, or VEH. Analyses of each 10-min block showed that differences between groups were most evident in the first half of the 60-min test session. Data are presented as mean + sem. Black star denotes VEH-ZOLP $<$ ZOLP-ZOLP and DZP-ZOLP groups $(p<0.05)$. Reprinted with permission. Wright, B.T., C.F. Gluszek, and S.A. Heldt, The effects of repeated ZOLP treatment on tolerance, withdrawal-like symptoms, and GABAA receptor $m R N A$ s profile expression in mice: comparison with DZP. Psychopharmacology (Berl), 2014. 231(15): p. 2967-79 [16]. 
For distance traveled in the entire open field, a two-way ANOVA found that there was a main effect of time, $F(5,105)=34.78, p<0.01$, a main effect of treatment, $F(2,21)$ $=8.08, p<0.01$, and a significant interaction of Time $\mathrm{x}$ Duration, $F(10,105)=2.97, p<$ 0.01 . To assess the interaction, a one-way ANOVAs at each level of time were performed. There were group differences at Block $1, F(2,21)=16.82, p<0.01$, Block 2 , $F(2,21)=8.25, p<0.01$, Block 3, $F(2,21)=4.29, p<0.05$, and Block 5, $F(2,21)=4.70$, $p<0.05$. Follow-up Dunnett's t-tests revealed that the activity of ZOLP and DZP groups was significantly lower than the VEH groups at Blocks 1 and 2, $p<0.01$ and the activity of DZP groups was significantly lower than the VEH groups at Blocks 3 and 5, $p<0.05$. There was no significantly differences at Blocks 4 and $6, F_{\mathrm{s}}(2,21)>3.11, p>0.05$ (Figure 4-5).

For percent of center zone time, a two-way ANOVA yielded main effects of treatment, $F(2,23)=5.27 ; p<0.02$, time, $F(5,115)=8.36 ; p<0.01$, and a significant Treatment $\times$ Time interaction, $F(10,115)=2.49 ; p=0.01$. One-way ANOVAs followed by Dunnett's $t$-tests indicated significant differences during Blocks 4-6, $F(2,23)=7.82, p$ $<0.01 ; F(2,23)=4.25, p<0.05$; and $F(2,23)=5.95, p<0.01$. There were no significant differences between groups at Blocks $1-3, F(2,23)<3.3, p>0.05$. Overall, ZOLP and DZP groups spent significantly less percent of time in the center zone in Blocks 4-6 compared to VEH mice, $p s<0.01$ (Figure 4-6).

For the number of center zone entries, a two-way ANOVA yielded, main effects of treatment, $F(2,23)=8.20 ; p<0.01$, and time, $F(5,115)=41.67 ; p<0.01$, were significant. The Treatment $\times$ Time interaction, $F(10,115)=2.67 ; p<0.01$ was also significant. ANOVAs followed by $t$-tests indicated that ZOLP and DZP groups had fewer center zone entries during Blocks 1 and 2 (Figure 4-6). Analyses of frequency of rearing showed reliable effects of time, $F(5,115)=6.28 ; p<0.01$, and treatment, $F(2,23)=$ 41.67; $p<0.01$. Overall, the frequency of rearing was lower in both ZOLP and DZP groups in comparison to the VEH group (Figure 4-6).

\section{Flumazenil-Precipitated Withdrawal}

Cohort 3 mice were used to assess the presence of flumazenil-precipitated withdrawal symptoms on two consecutive days (Day1 and Day 2) after vehicle, diazepam, or zolpidem treatments. On Day 1, mice were given flumazenil $(10 \mathrm{mg} / \mathrm{kg} / \mathrm{i} . \mathrm{p})$ 20 min before EPM and subsequent open field testing. One-way ANOVA analyses of EPM measures revealed no significant differences between groups for percent time in the open arms or entries into the open arms, $F_{\mathrm{s}}(2,10)<1.38$, $p \mathrm{~s}>0.05$ (Figure 4-7) Group differences in Day 1 open field dependent measures were assessed using two-way ANOVAs. For distance traveled, this analysis indicated a significant effect of time, $F(5,45)=52.21, p<0.01$, and a significant interaction of Time $\mathrm{x}$ Treatment, $\mathrm{F}(10,45)=$ $2.18, p<0.05$. There was no significant effect of treatment, $F(2,9)=1.13, p>0.05$. A one-way ANOVA found significant group differences at Block 6, with ZOLP and DZP groups displaying less activity than the VEH group, $p \mathrm{~s}<0.05$ (Figure 4-8). For percent time spent in the open field center zone, analysis indicated a significant effect of time, 


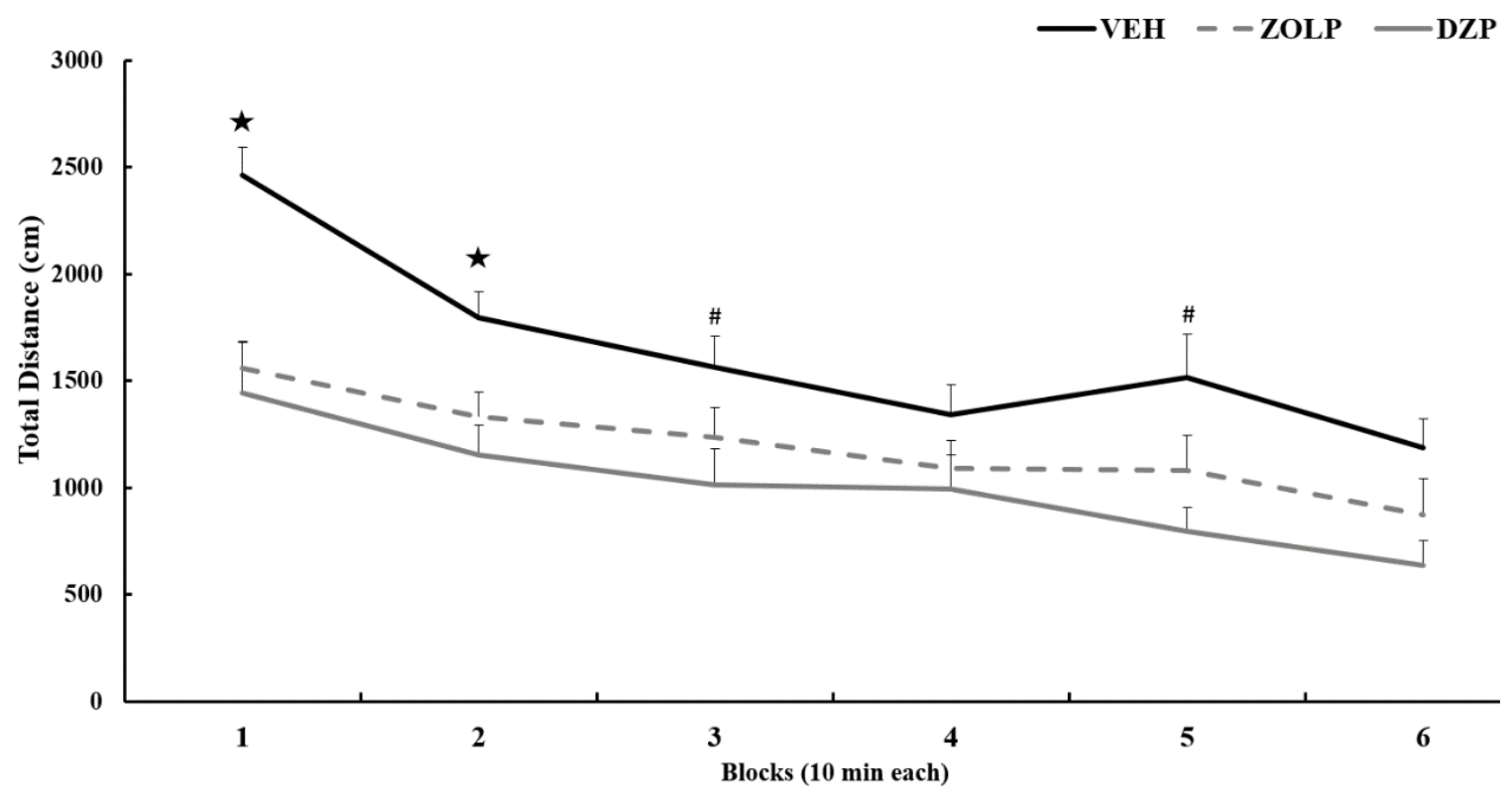

Figure 4-5. Decreased locomotor activity in zolpidem and diazepam groups after cessation of 7 days of zolpidem and diazepam

Distance traveled in open field after test administration of VEH in mice previously given subchronic treatment of ZOLP, DZP, or VEH. Analyses of each 10-min block showed that differences between groups were most evident in the first half of the 60- min test session. Data are presented as mean + sem. Black star denotes VEH $>$ ZOLP and DZP groups $(p<0.05)$. Number sign denotes VEH $>$ DZP group $(p<0.05)$. Reprinted with permission. Wright, B.T., C.F. Gluszek, and S.A. Heldt, The effects of repeated ZOLP treatment on tolerance, withdrawal-like symptoms, and GABAA receptor $m R N A$ s profile expression in mice: comparison with DZP. Psychopharmacology (Berl), 2014. 231(15): p. $2967-79$ [16]. 

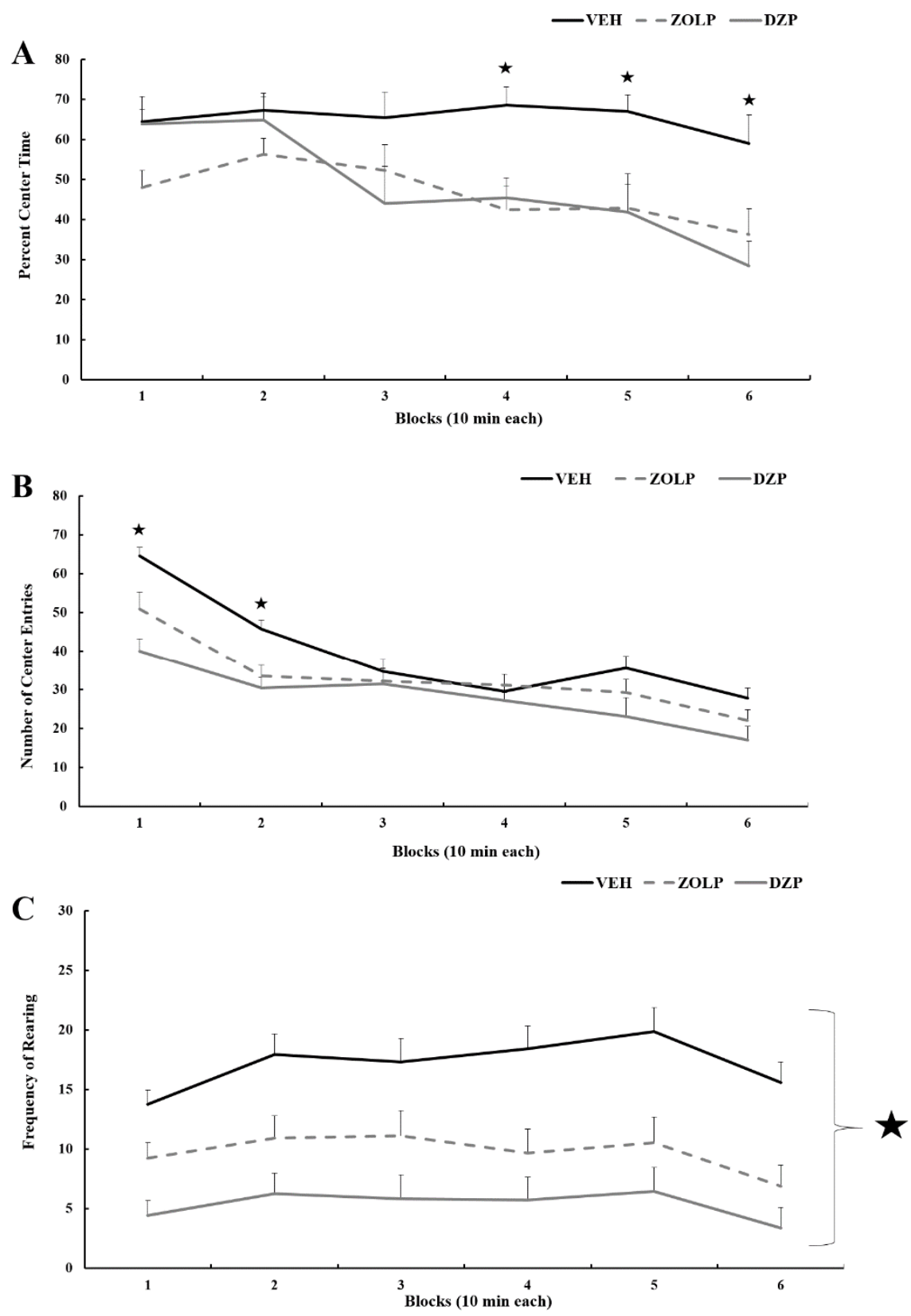

Figure 4-6. Decreased percent time in center, center entries, and rearing frequency after cessation of 7 days of zolpidem and diazepam

Multiple exploratory behaviors were decreased following cessation of drug. (A) Percent of center time, (B) Number of center entries, and (C) Rearing frequency. Analyses were conducted on means of each 10-min block. Data are presented as mean + sem. Black star denotes VEH significantly different than ZOLP and DZP groups $(p<0.05)$. Reprinted with permission. Wright, B.T., C.F. Gluszek, and S.A. Heldt, The effects of repeated ZOLP treatment on tolerance, withdrawal-like symptoms, and GABAA receptor $m R N A s$ profile expression in mice: comparison with DZP. Psychopharmacology (Berl), 2014. 231(15): p. 2967-79 [16]. 

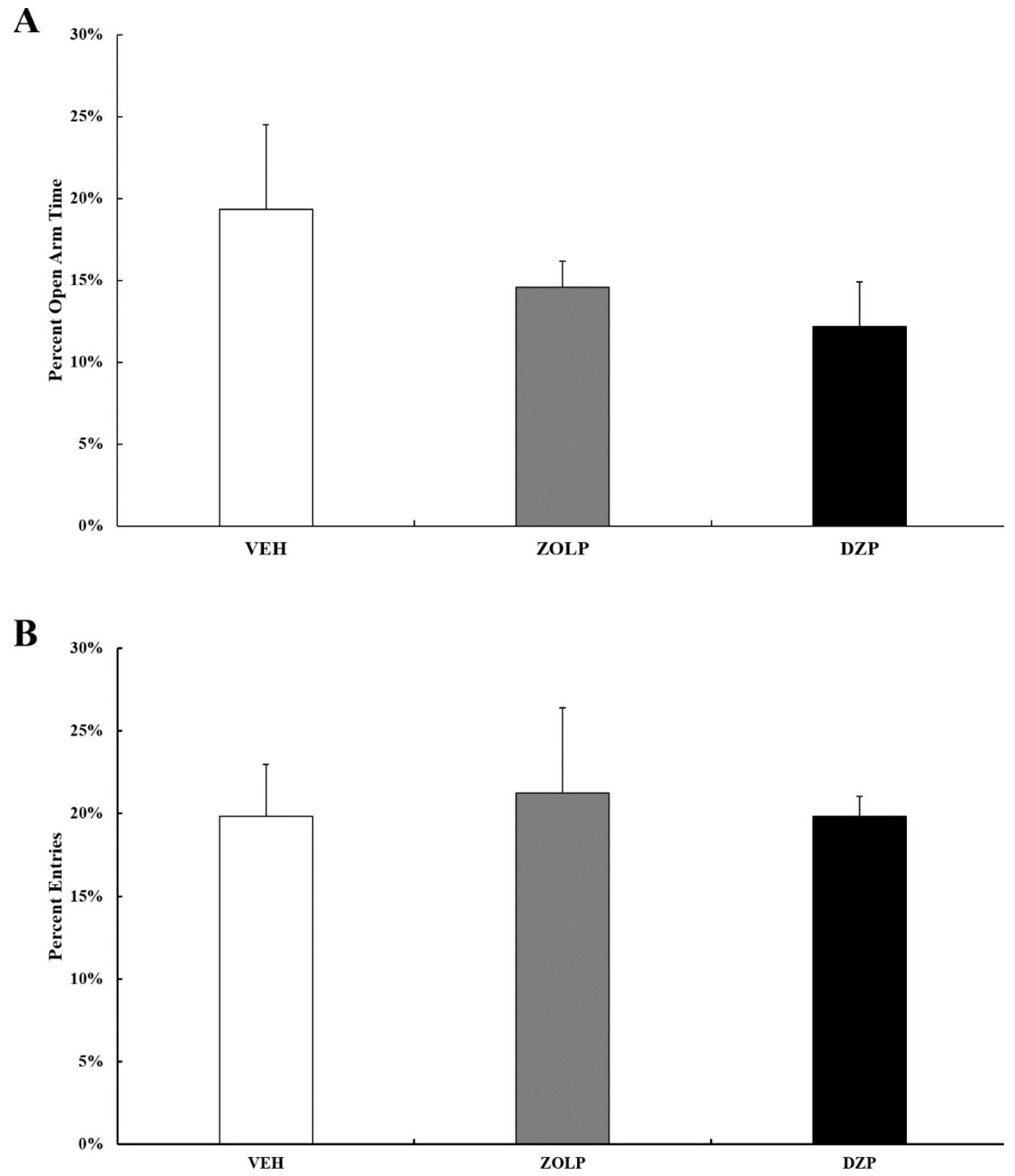

Figure 4-7. Percent time spent and percent entries into the open arms of the EPM after acute flumazenil administration after 7-day administration of zolpidem, diazepam, or vehicle

Histogram comparing (A) percent of time spent in the open arms and (B) percent entries into the EPM. Statistical analyses were performed by ANOVA with Dunnett's $t$-test for pairwise comparisons against a VEH. Data are presented as mean + sem. There were no significant differences between the groups $(p>0.05)$. 


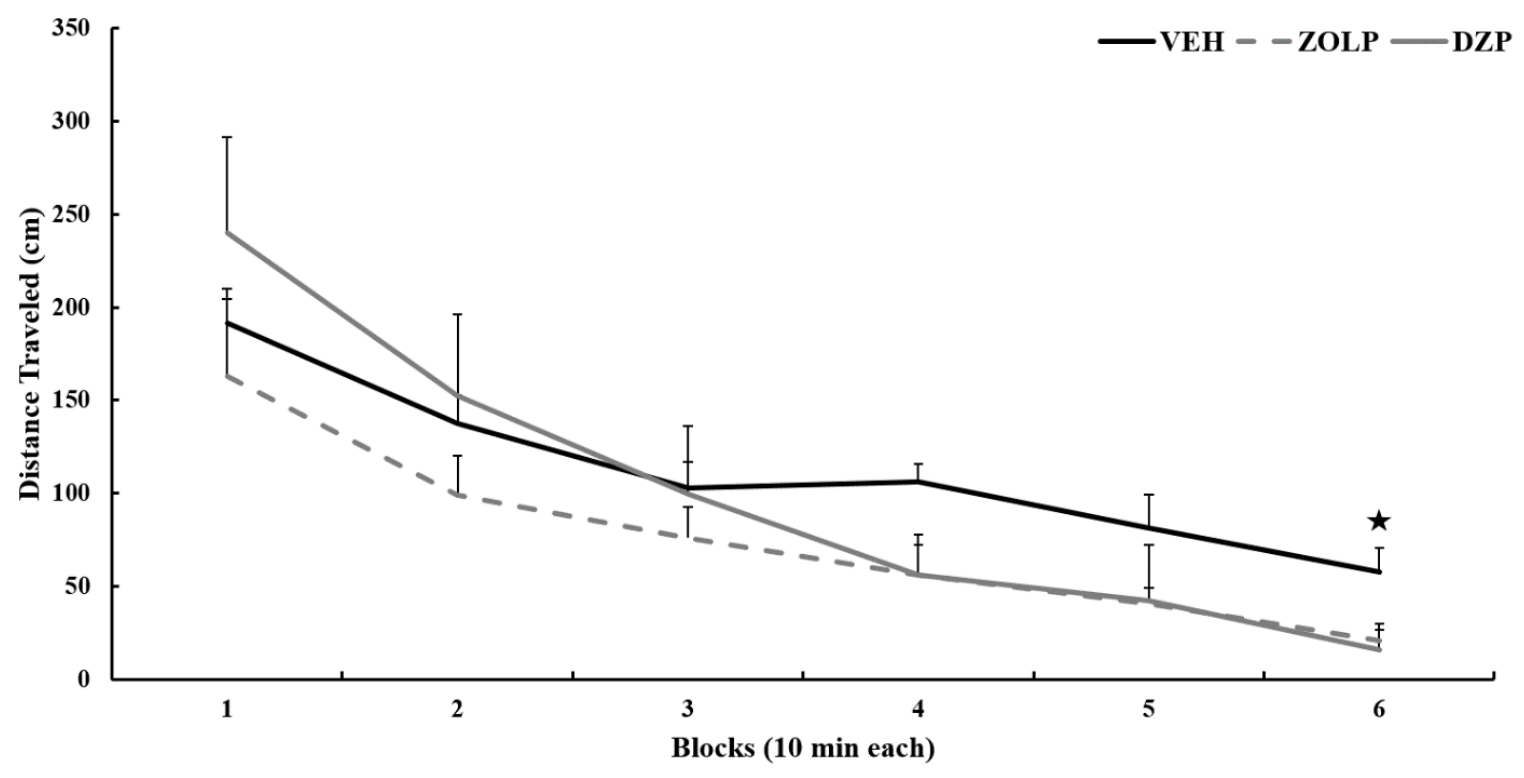

Figure 4-8. Locomotor effect of flumazenil on test day 1 after 7-days administration of zolpidem, diazepam, or vehicle

Distance traveled in open field after test administration of FLU in mice previously given subchronic treatment of ZOLP, DZP, or VEH on test day 1. Analyses were conducted on means of each 10- min block. There was a significant decrease in activity at Block 6 on day 1 , but no significant differences between groups on day 2. Data are presented as mean + sem. Black star denotes VEH significantly different than ZOLP and DZP groups $(p<0.05)$. 
$F(5,45)=45.41, p<0.01$, but no significant effect of treatment, $F(2,9)=0.83, p>0.05$, or a significant interaction of Time $\mathrm{x}$ Treatment, $F(10,45)=1.31, p>0.05$ (Figure 4-9). For center entries, the analysis indicated a significant effect of time, $F(5,45)=22.70, p<$ 0.01 , but no significant effect of treatment, $F(2,9)=2.83, p>0.05$, or a significant interaction of Time $\mathrm{x}$ Treatment, $F(10,45)=0.38, p>0.05$ (Figure 4-9). For frequency of rearing on Day 1, analysis indicated a significant effect of time, $F(5,45)=23.01, p<$ 0.01 , but no significant effect of treatment, $F(2,9)=1.54, p>0.05$, or a significant interaction of Time $\mathrm{x}$ Treatment, $F(10,45)=0.40, p>0.05$ (Figure 4-9). $0.05)$. Data are presented as mean + sem.

On Day 2, mice were given of flumazenil (10 mg/kg/i.p) $20 \mathrm{~min}$ before open field testing, and group differences in dependent measures were assessed using two-way ANOVAs. The analysis of group difference in distance traveled showed a significant effect of time, $F(5,50)=18.76, p<0.01$, but no significant effect of treatment, $\mathrm{F}(2,10)=$ $0.627, p>0.05$ or a significant interaction of Time $\mathrm{x}$ Treatment, $F(10,50)=0.933, p>$ 0.05 (Figure 4-10). For percent time spent in center on Day 2, analysis indicated a significant effect of time, $F(5,50)=4.41, p<0.01$ but no significant effect of treatment, $F(2,10)=0.791, p>0.05$ or significant interaction of Time $\mathrm{x}$ Treatment, $F(10,50)=0.21$, $p>0.05$ (Figure 4-11). For center entries, analysis indicated a significant effect of time, $F(5,50)=20.03, p<0.01$, and a significant interaction of Time $\mathrm{x}$ Treatment, $F(10,50)=$ $3.33, p>0.01$, but no significant effect of treatment, $F(2,10)=0.61, p>0.05$. A one-way ANOVA found significant group differences at Block $1, F(2,10)=4.38, p<0.05$, with the ZOLP group having less center entries than the VEH groups (Figure 4-11). There were no significant group differences at Blocks $2-6, F(2,10)<4.32, p>0.05$. For frequency of rearing, $\mathrm{t}$ analysis indicated a significant effect of time, $F(5,50)=5.46, p<$ 0.01 but no significant effect of treatment $F(2,10)=0.45, p>0.05$ or a significant interaction of Time $\mathrm{x}$ Treatment, $F(10,50)=2.01, p>0.05$ (Figure 4-11).

\section{Changes in mRNA Expression Measured by qRT-PCR}

Cohort 4 was used to measure changes in mRNA expression of $\mathrm{GABA}_{\mathrm{A}} \mathrm{R}$ subunits and related proteins in the cortex, hippocampus, amygdala, and PFC. In the cortex (Figure 4-12), a one-way ANOVA identified group differences for the subunits $\alpha 1, F(2,17)=12.97, p<0.01 ; \alpha 3, F(2,17)=6.92, p<0.01 ; \beta 2, F(2,17)=5.80, p<$ 0.05 , and $\delta, F(2,17)=5.93, p<0.05$. Follow-up Dunnett's t tests revealed decreased expression levels of $\alpha 1, \alpha 3, \beta 2$, and $\delta$ subunits in ZOLP and DZP groups compared to VEH $(p s<0.05)$ and DZP groups but not ZOLP groups, induced significant decreases in expression of $\alpha 4, \alpha 5, \beta 1$, and $\gamma 2$ subunits after chronic treatment $(p s<0.05)$. In addition to subunits, group differences were identified for gephyrin, $F(2,17)=4.95, p<0.05$, and GAT-1, $F(2,17)=5.80, p<0.05$.There were no significant differences for the other subunits, $F \mathrm{~s}(2,17)<4.39$, $p \mathrm{~s}>0.05$.

In the hippocampus (Figure 4-13), a one-way ANOVA identified group differences for the $\alpha 5$ subunit, $F(2,18)=3.82, p<0.05$ and $\gamma 2$ subunit, $F(2,17)=5.22, p$ $<0.05$. Follow-up Dunnett's t tests revealed decreased expression levels of $\alpha 5$ and $\gamma 2$ 

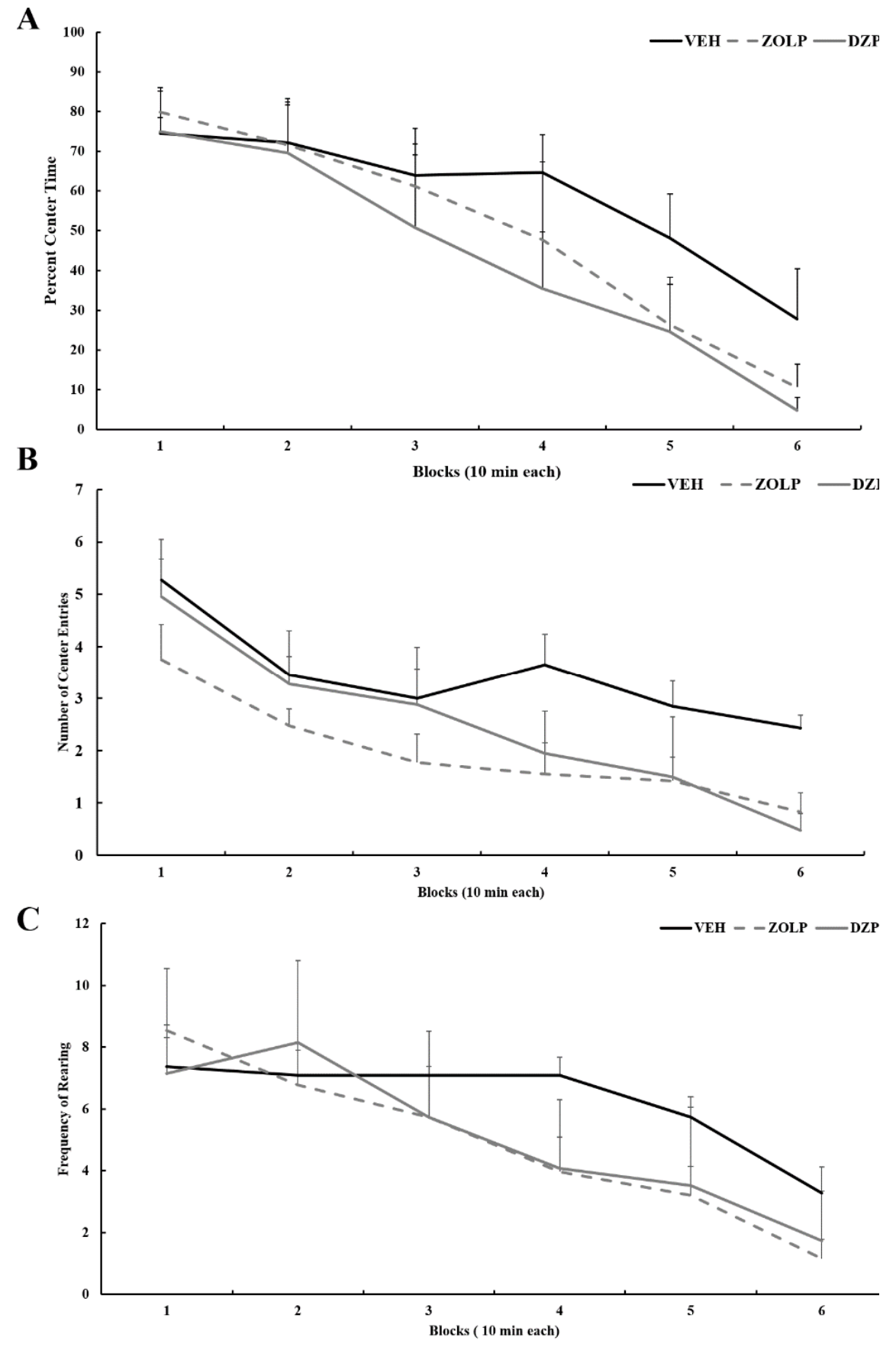

Figure 4-9. Effect of flumazenil on percent time in center, center entries, and rearing frequency on test day 1 after 7-days administration of zolpidem, diazepam, or vehicle

Percent time in center, center entries, and rearing frequency in open field after test administration of FLU in mice previously given subchronic treatment of ZOLP, DZP, or $\mathrm{VEH}$ on test day 1. Analyses were conducted on means of each 10- min block. There were no significant differences between the groups on day $1(p>0.05)$. Data are presented as mean + sem. 


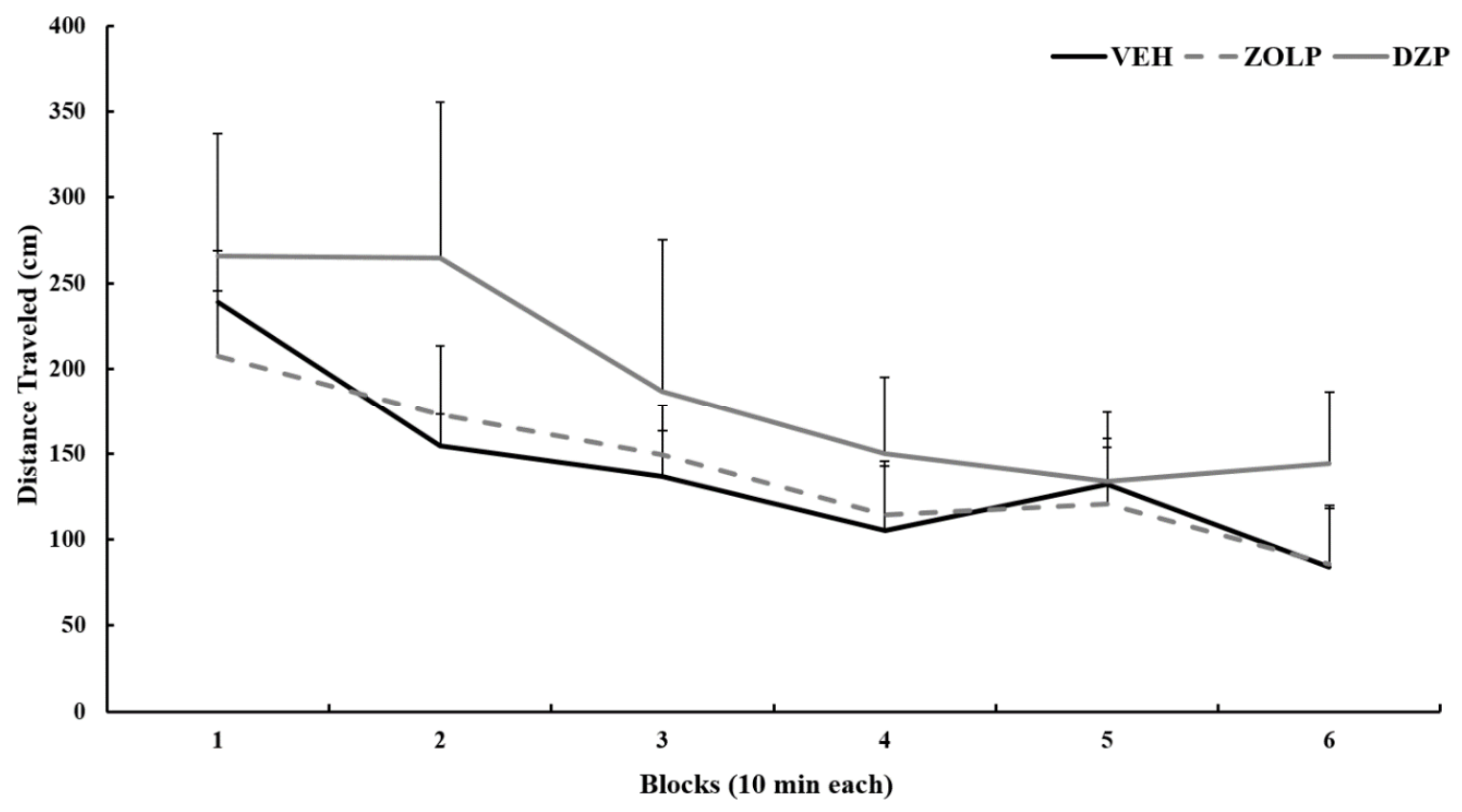

Figure 4-10. Locomotor effect of flumazenil test day 2 after 7-day administration of zolpidem, diazepam, or vehicle

Distance traveled in open field after test administration of FLU in mice previously given subchronic treatment of ZOLP, DZP, or VEH day 2. Analyses were conducted on means of each 10- min block. There were no significant differences between groups, $p>0.05$. Data are presented as mean + sem. 

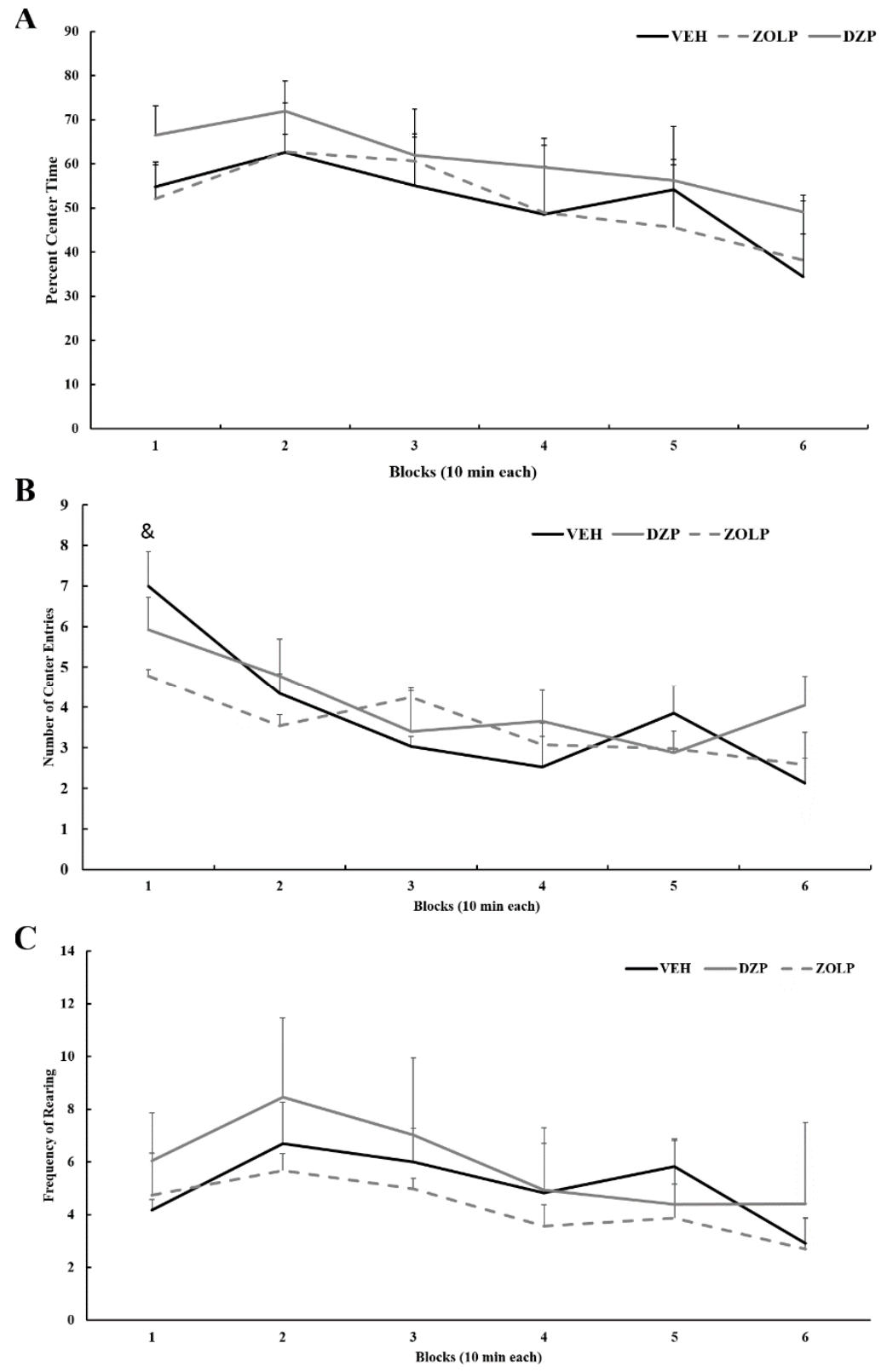

Figure 4-11. Effect of flumazenil on percent time in center, center entries, and rearing frequency on test day 2 after 7-days administration of zolpidem, diazepam, or vehicle

Percent time in center, center entries, and rearing frequency in open field after test administration of FLU in mice previously given subchronic treatment of ZOLP, DZP, or $\mathrm{VEH}$ on test day 2. Analyses were conducted on means of each 10- min block. There were significant group differences on the number of center entries at Block 1. ZOLP groups had less center entries than VEH groups. There were no significant group differences at other measures, $p>0.05$. Data are presented as mean + sem. Ampersnad denotes VEH significantly different than ZOLP and DZP groups $(p<0.05)$. 


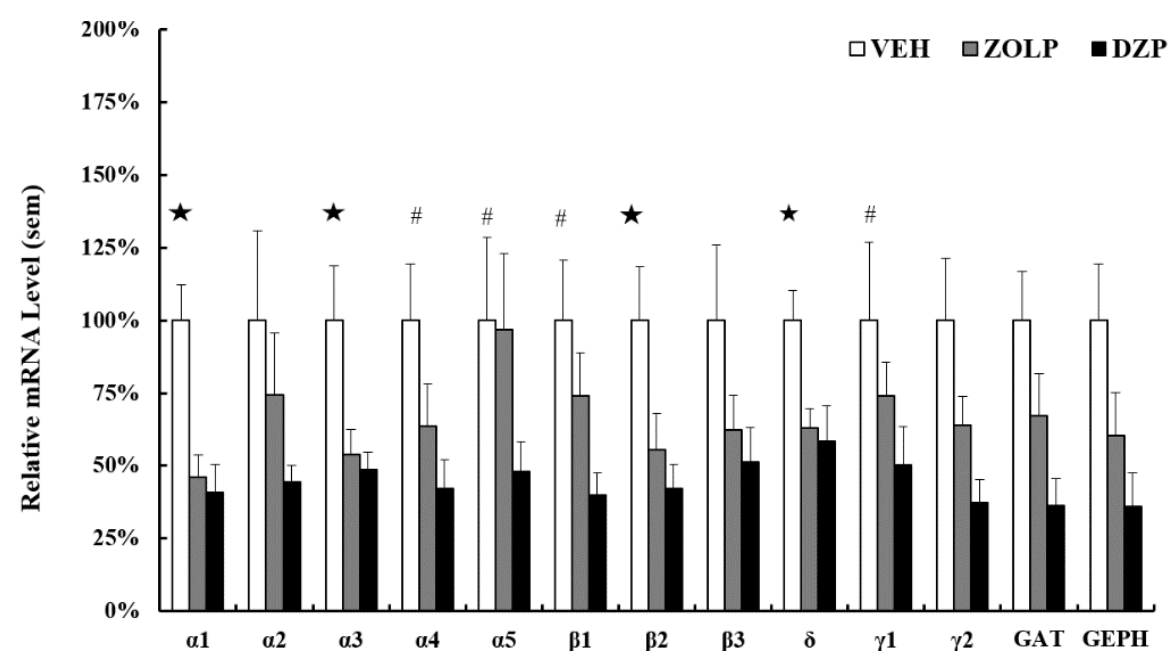

Figure 4-12. Relative levels of mRNA in the cortex following 7-day administration of zolpidem, diazepam, or vehicle

Histograms comparing levels of transcripts are expressed as mean percentage of VEH control $(100 \%)$. Statistical analyses were performed by ANOVA with Dunnett's $t$ test for pairwise comparisons against a VEH. Data are presented as mean + sem. Black star denotes VEH significantly different than ZOLP and DZP treatments $(p<0.05)$; Number sign denotes VEH significantly different than DZP treatment $(p<0.05)$. Reprinted with permission. Wright, B.T., C.F. Gluszek, and S.A. Heldt, The effects of repeated ZOLP treatment on tolerance, withdrawal-like symptoms, and GABAA receptor $m R N A$ s profile expression in mice: comparison with DZP. Psychopharmacology (Berl), 2014. 231(15): p. $2967-79[16]$. 


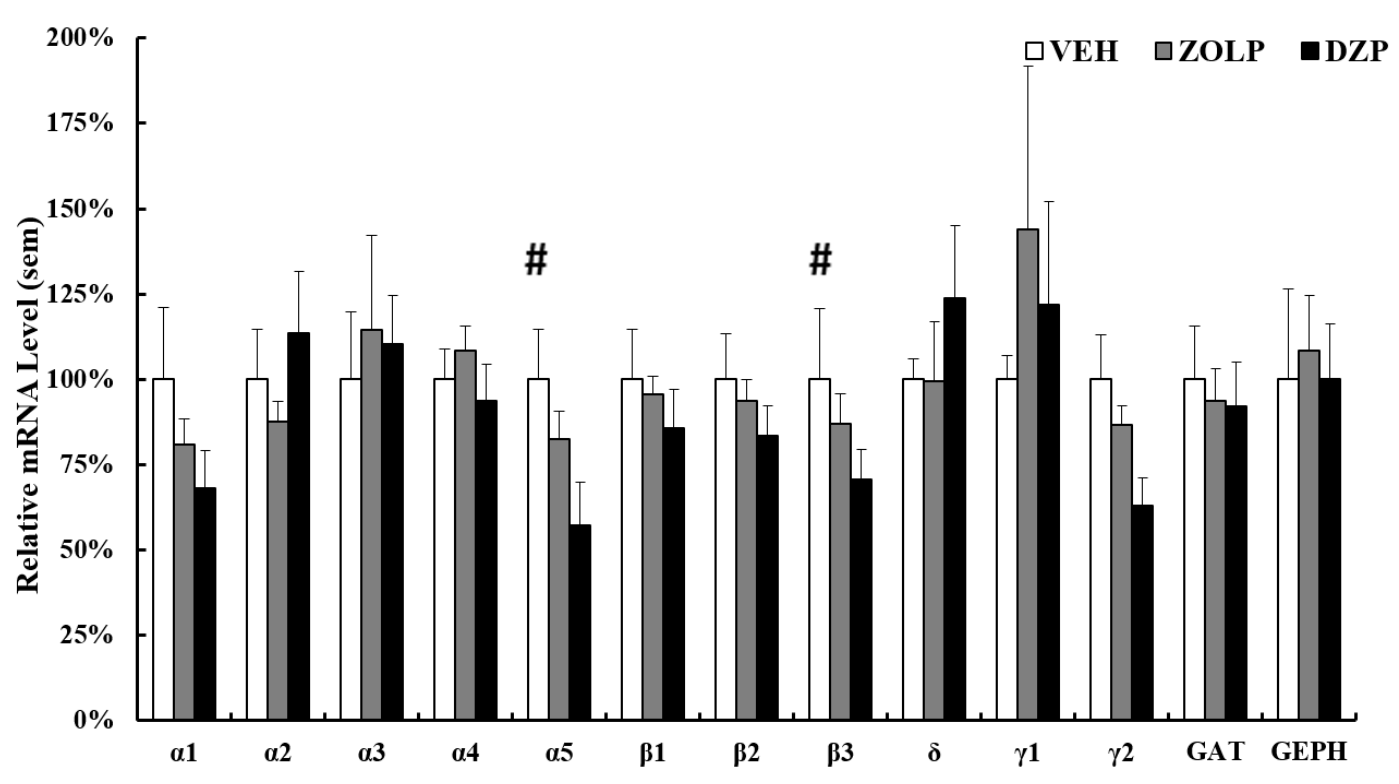

Figure 4-13. Relative levels of mRNA in the hippocampus following 7-day administration of zolpidem, diazepam, or vehicle

Histograms comparing levels of transcripts are expressed as mean percentage of VEH control (100\%). Statistical analyses were performed by ANOVA with Dunnett's $t$ test for pairwise comparisons against a VEH. Data are presented as mean + sem. Black star denotes VEH significantly different than ZOLP and DZP treatments $(p<0.05)$; Number sign denotes VEH significantly different than DZP treatment $(p<0.05)$. Reprinted with permission. Wright, B.T., C.F. Gluszek, and S.A. Heldt, The effects of repeated ZOLP treatment on tolerance, withdrawal-like symptoms, and GABAA receptor $m R N A$ s profile expression in mice: comparison with DZP. Psychopharmacology (Berl), 2014. 231(15): p. $2967-79[16]$. 
subunits in DZP groups compared to VEH groups $(p \mathrm{~s}<0.05)$. There were no significant differences for the other subunits, $F_{\mathrm{S}}(2,18)<5.22, p \mathrm{~s}>0.05$. There were no significant group differences in the amygdala, $F_{\mathrm{s}}(2,20)<1.90, p \mathrm{~s}>0.05$, or the $\mathrm{PFC}, F_{\mathrm{s}}(2,20)<$ $0.95, p s>0.05$ for the other subunits (Figure 4-14).

The mRNA expression of AMPAR and NMDAR subunits in the cortex, hippocampus, amygdala, and PFC was also measured in Cohort 4. In the hippocampus, a one-way ANOVA showed significant differences between groups for GluN2A, $F(2,16)=$ $4.650, p<0.05$. Follow-up Dunnett's $t$-test found that there was a decrease in GluN2A in the DZP group compared to the VEH group, $p<0.05$ (Figure 4-15). There were no significant group differences for other AMPA or NMDA subunits in the hippocampus. Likewise, there were no group differences in the cortex, $F \mathrm{~s}(2,12)<0.625, p \mathrm{~s}>0.05$; amygdala, $F \mathrm{~s}(2,16)<0.76, p \mathrm{~s}>0.05$; or the PFC, $F \mathrm{~s}(2,20)<1.73, p \mathrm{~s}>0.05$ for the other subunits (Figure 4-16).

\section{Changes in Total and Surface Subunit Protein Expression}

Cohort 5 mice who were given previous zolpidem, diazepam, and vehicle treatment were used to measure differences in cortical and hippocampal protein expression. In the cortex, one-way ANOVAs found significant differences between groups for total $\alpha 2$ subunit, $F(2,15)=4.95, p<0.05$, and $\gamma 2, F(2,6)=18.16, p<0.01$. In the cortex, Dunnett's $t$-tests found that total $\alpha 2$ subunit expression is decreased in ZOLP mice and total $\gamma 2$ subunit expression is decreased in DZP mice (Figures 4-17 and 4-18). ANOVAs revealed no groups differences in total $\alpha 1, F(2,15)=2.47, p>0.05$; total $\alpha 3, F$ $(2,6)=1.30, p>0.05$; total GluN1, $F(2,5)=0.235, p>0.05$; and total GluN2B, $F(2,5)=$ $2.50, p>0.05$ (Figures 4-19 through 4-22). A one-way ANOVA found significant differences between groups for intracellular $\alpha 1, F(2,6)=5.49, p<0.05$ and intracellular $\alpha 1$ was decreased in DZP groups, $p<0.05$. A one-way ANOVA found no group differences with surface $\alpha 1$ subunits $F(2,6)=0.176, \mathrm{p}>0.05$, (Figure 4-23). In the hippocampus one-way ANOVAs found no significant differences between groups for total $\alpha 1, F(2,5)=2.72, p>0.05$ or total $\alpha 2, F(2,5)=3.37, p>0.05$ (Figures $4-24$ and 4-25).

In addition to the parametric tests, non-parametric tests were run for the data sets for total $\alpha 1$ in the cortex and total $\alpha 2$ in the hippocampus did not meet the assumptions needed for conducting parametric tests, therefore non-parametric tests were used for analysis. Non-parametric tests using Kruskal-Wallis $\mathrm{H}$ test showed that there were no significant differences in protein expression between the treatment groups for total $\alpha 1$, $\chi^{2}(2)=4.82, p>0.05$, and for total $\alpha 2, \chi^{2}(2)=5.14, p>0.05$.

In summary, mice previously given zolpidem or diazepam treatment for 7 days were less sensitive to the sedative effects of zolpidem treatment when compared to vehicle mice, indicating the presence of tolerance and cross-tolerance, respectively. Zolpidem and diazepam treated mice given vehicle injections prior to testing showed evidence of spontaneous withdrawal. Specifically, these mice displayed anxiety-like 


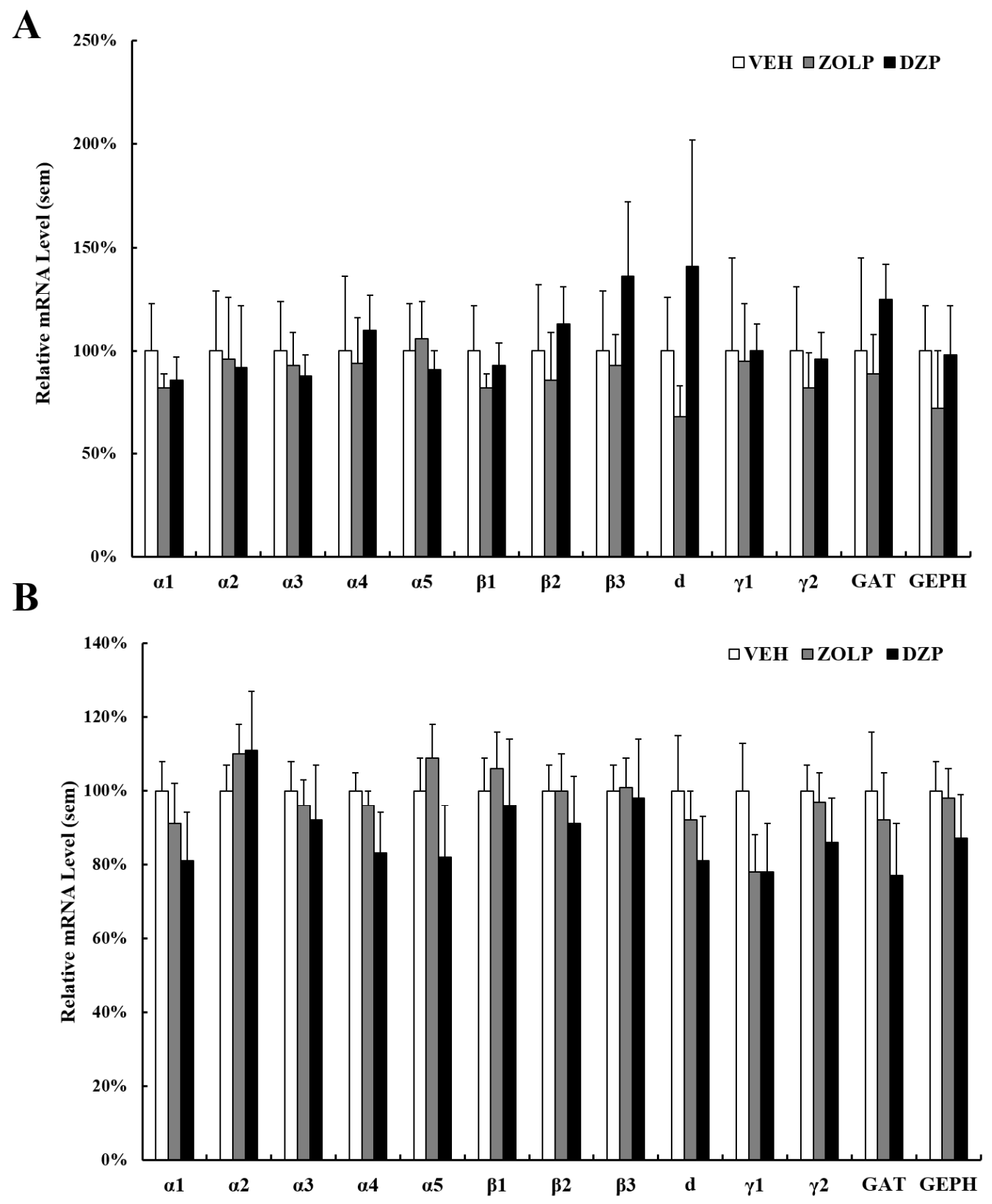

Figure 4-14. Relative levels of mRNA in the amygdala and pfe following 7-day administration of zolpidem, diazepam, or vehicle

Histograms comparing levels of transcripts are expressed as mean percentage of VEH control (100\%). Statistical analyses were performed by ANOVA with Dunnett's $t$ test for pairwise comparisons against a VEH in the (A) amygdala and (B) pfc. Data are presented as mean + sem. There were no significant differences between groups, $p \mathrm{~s}>0.05$. Reprinted with permission. Wright, B.T., C.F. Gluszek, and S.A. Heldt, The effects of repeated ZOLP treatment on tolerance, withdrawal-like symptoms, and GABAA receptor $m R N A s$ profile expression in mice: comparison with DZP. Psychopharmacology (Berl), 2014. 231(15): p. 2967-79 [16]. 


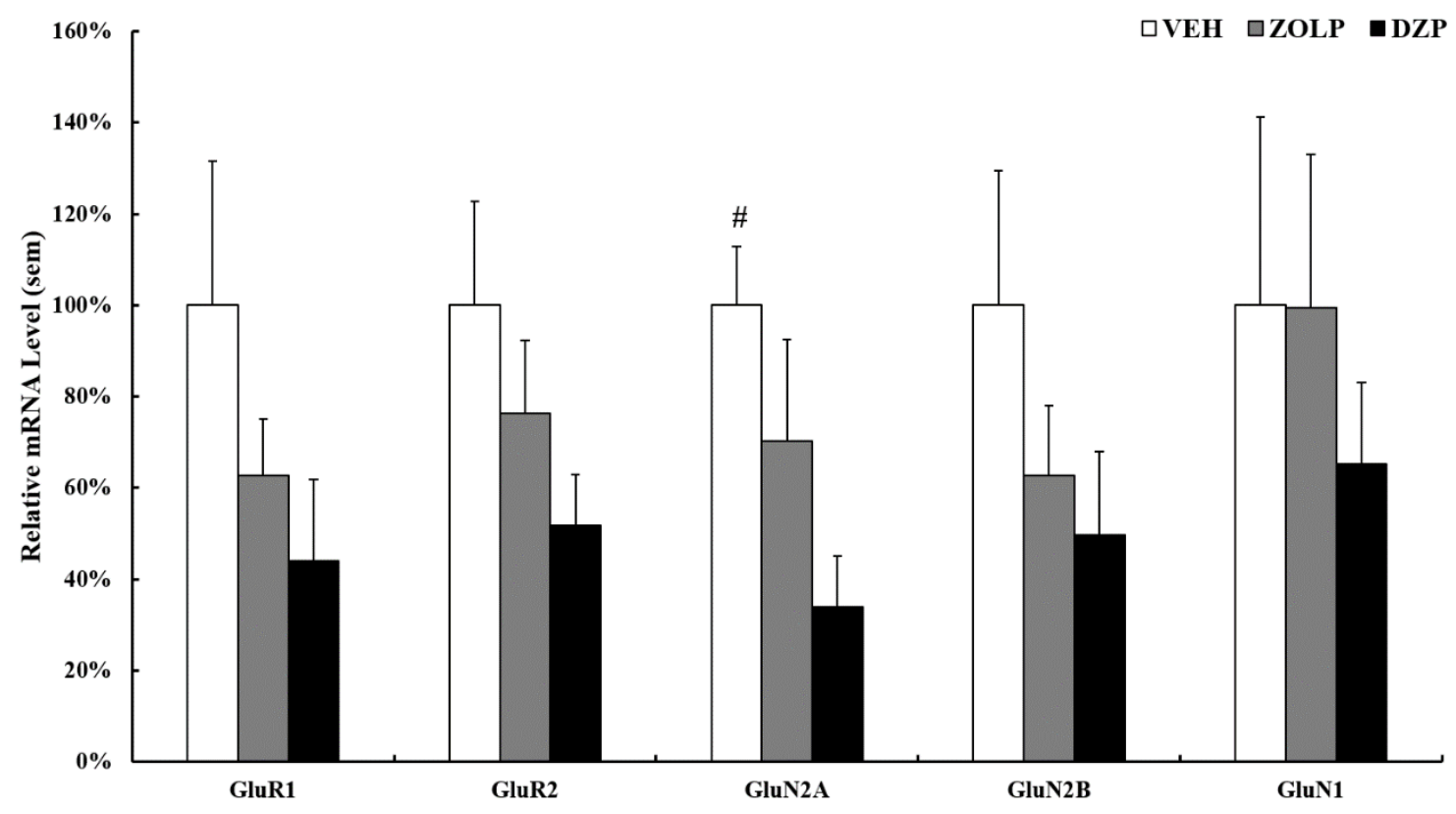

Figure 4-15. Relative levels of mRNA in the hippocampus following 7-day administration of zolpidem, diazepam, or vehicle

Histograms comparing levels of transcripts are expressed as mean percentage of VEH control $(100 \%)$. Statistical analyses were performed by ANOVA with Dunnett's $t$ test for pairwise comparisons against a VEH. Data are presented as mean + sem. Number sign denotes VEH significantly different than DZP treatment $(p<0.05)$. 

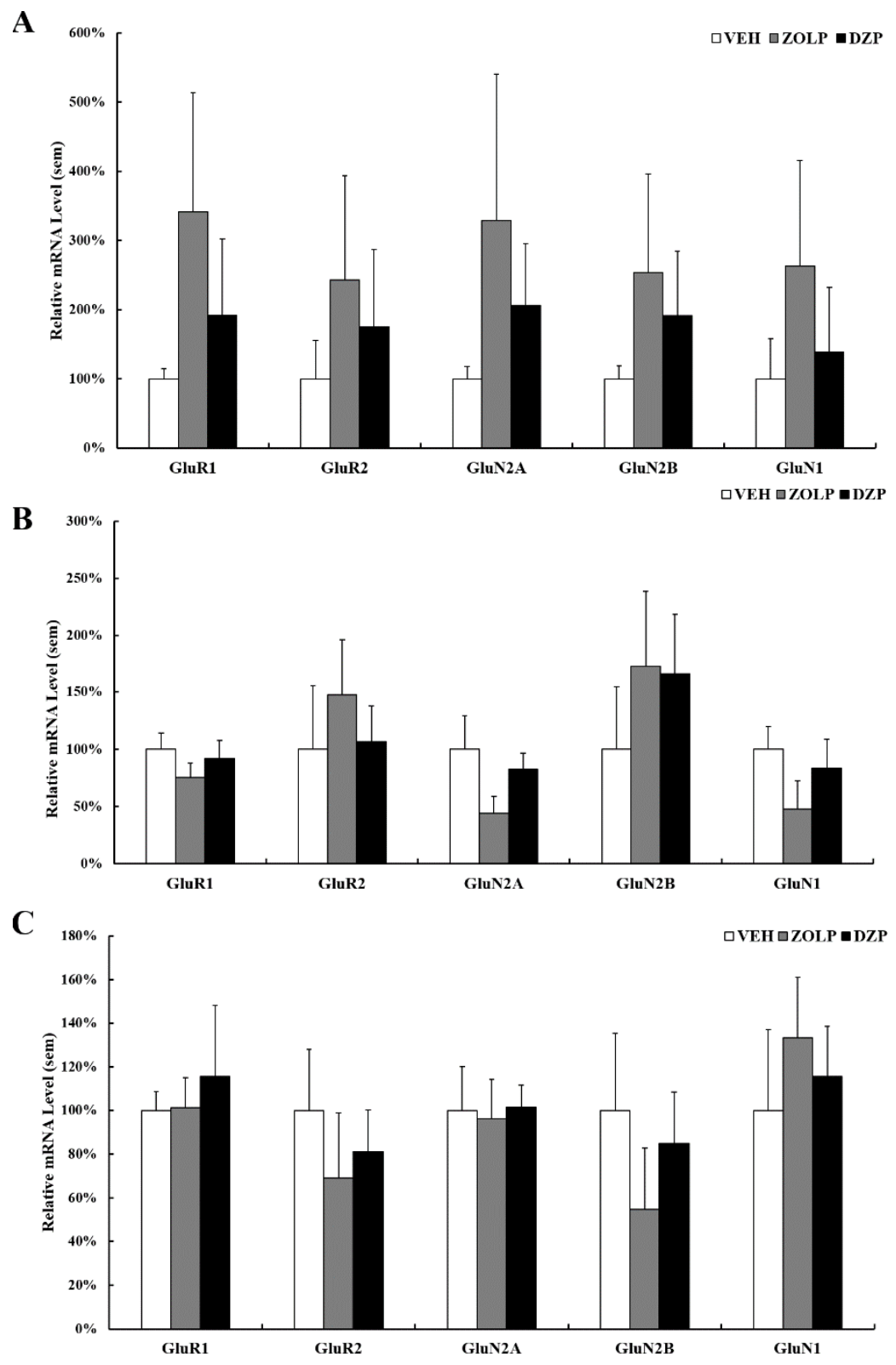

Figure 4-16. Relative levels of mRNA in the cortex, amygdala, and pfe following 7day administration of zolpidem, diazepam, or vehicle

Relative expression of protein from each group.Histograms comparing levels of transcripts are expressed as mean percentage of VEH control (100\%). Statistical analyses were performed by ANOVA with Dunnett's $t$ test for pairwise comparisons against a $\mathrm{VEH}$ in the (A) cortex, (B) amygdala, and (C) pfc. Data are presented as mean + sem. There were no significant differences between groups, $p \mathrm{~s}>0.05$. 


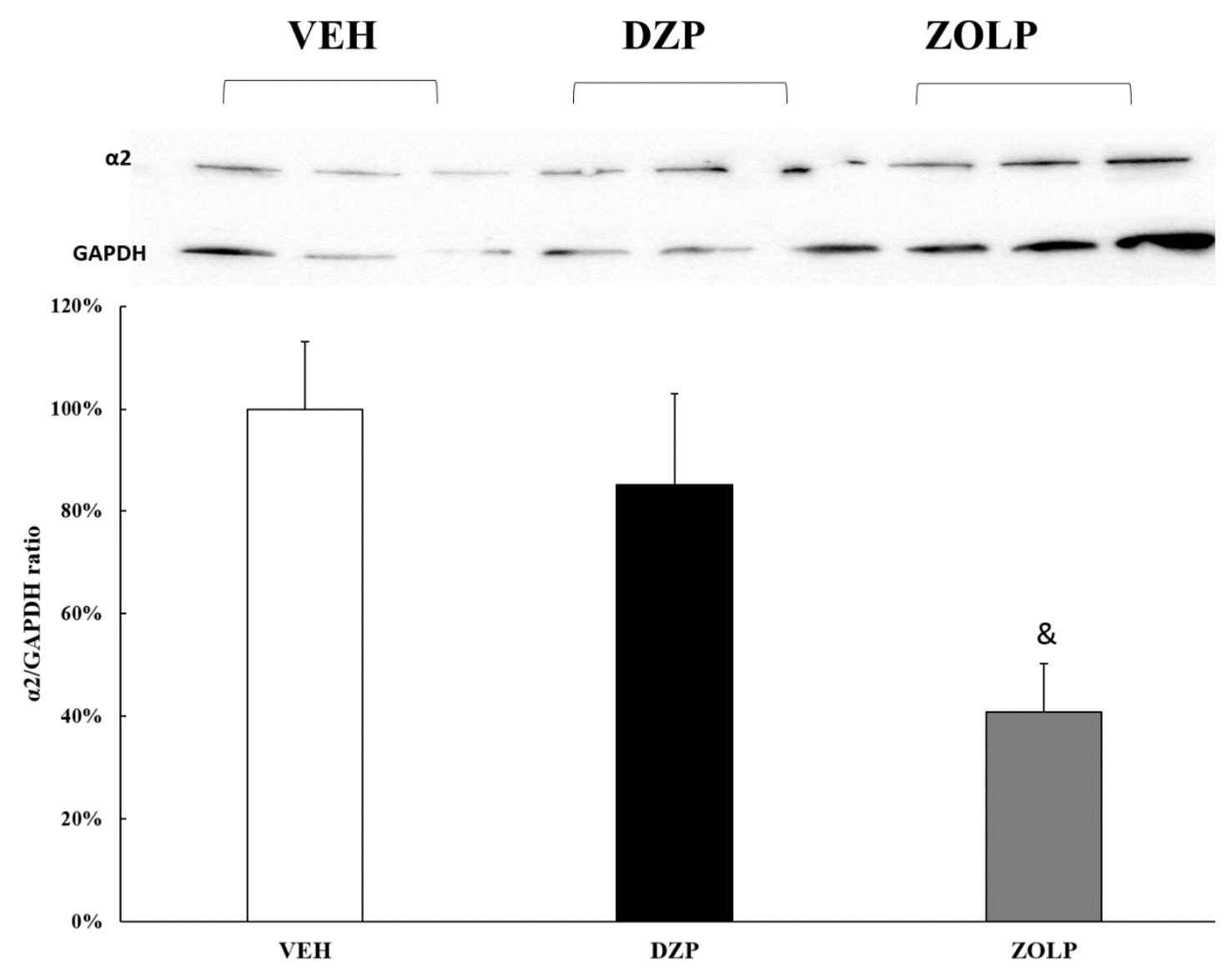

Figure 4-17. Relative $\alpha 2$ subunit expression in the cortex following 7-day administration of zolpidem, diazepam, or vehicle

Relative expression of protein from each group. Histograms comparing levels of transcripts are expressed as mean percentage of VEH control $(100 \%)$. Statistical analyses were performed by ANOVA with Dunnett's t test for pairwise comparisons against a VEH. Data are presented as mean + sem. There were significant differences between groups, $p$ s $<0.05$. The ZOLP group had less $\alpha 2$ protein expression the VEH group, $p<$ 0.05 . 


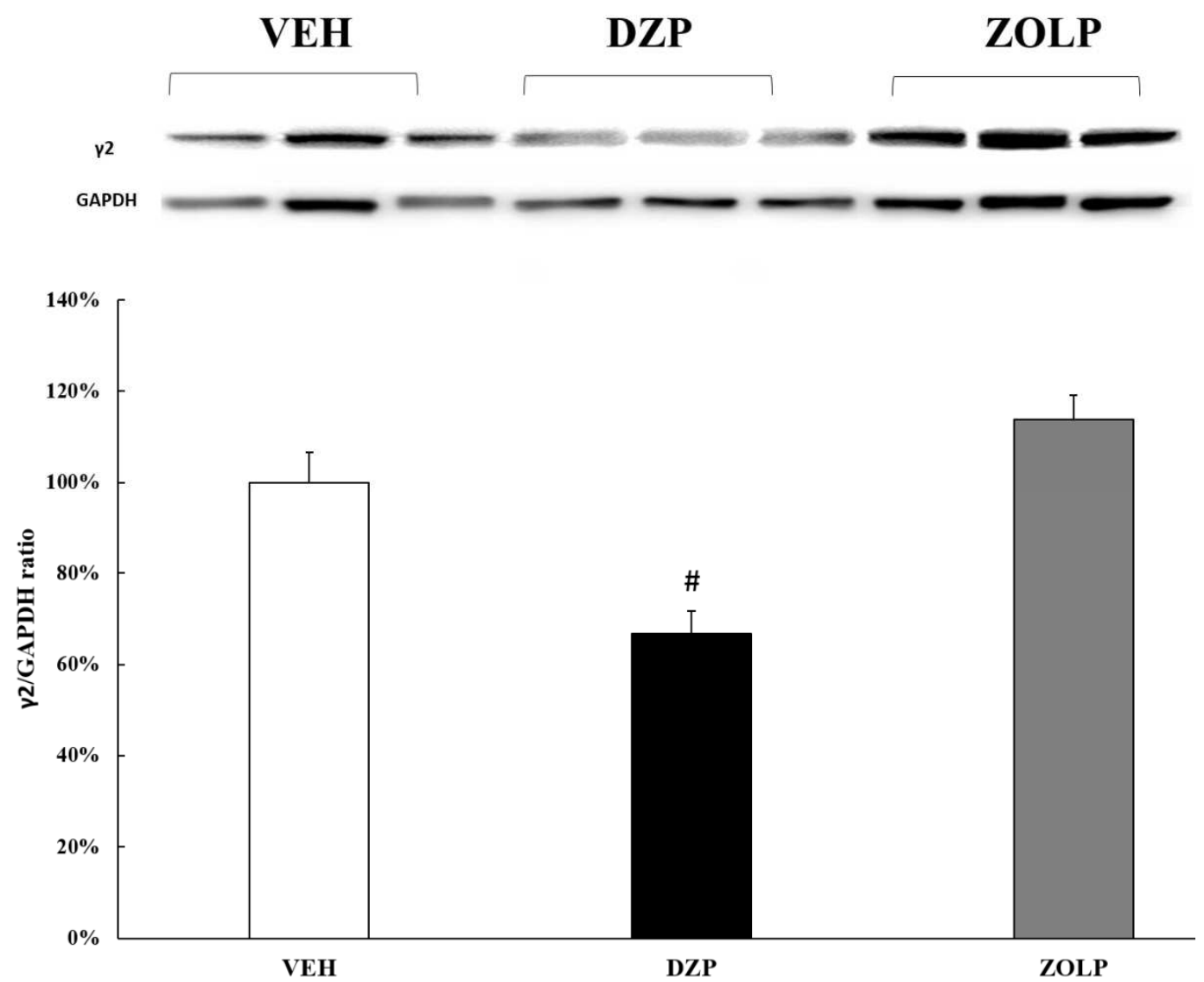

Figure 4-18. Relative $\gamma 2$ subunit expression in the cortex following 7-day administration of zolpidem, diazepam, or vehicle

Relative expression of protein from each group. Histograms comparing levels of transcripts are expressed as mean percentage of VEH control $(100 \%)$. Statistical analyses were performed by ANOVA with Dunnett's t test for pairwise comparisons against a VEH. Data are presented as mean + sem. There were significant differences between groups, $p \mathrm{~s}<0.05$. The DZP group had less $\gamma 2$ protein expression the $\mathrm{VEH}$ group, $p<$ 0.05 . 


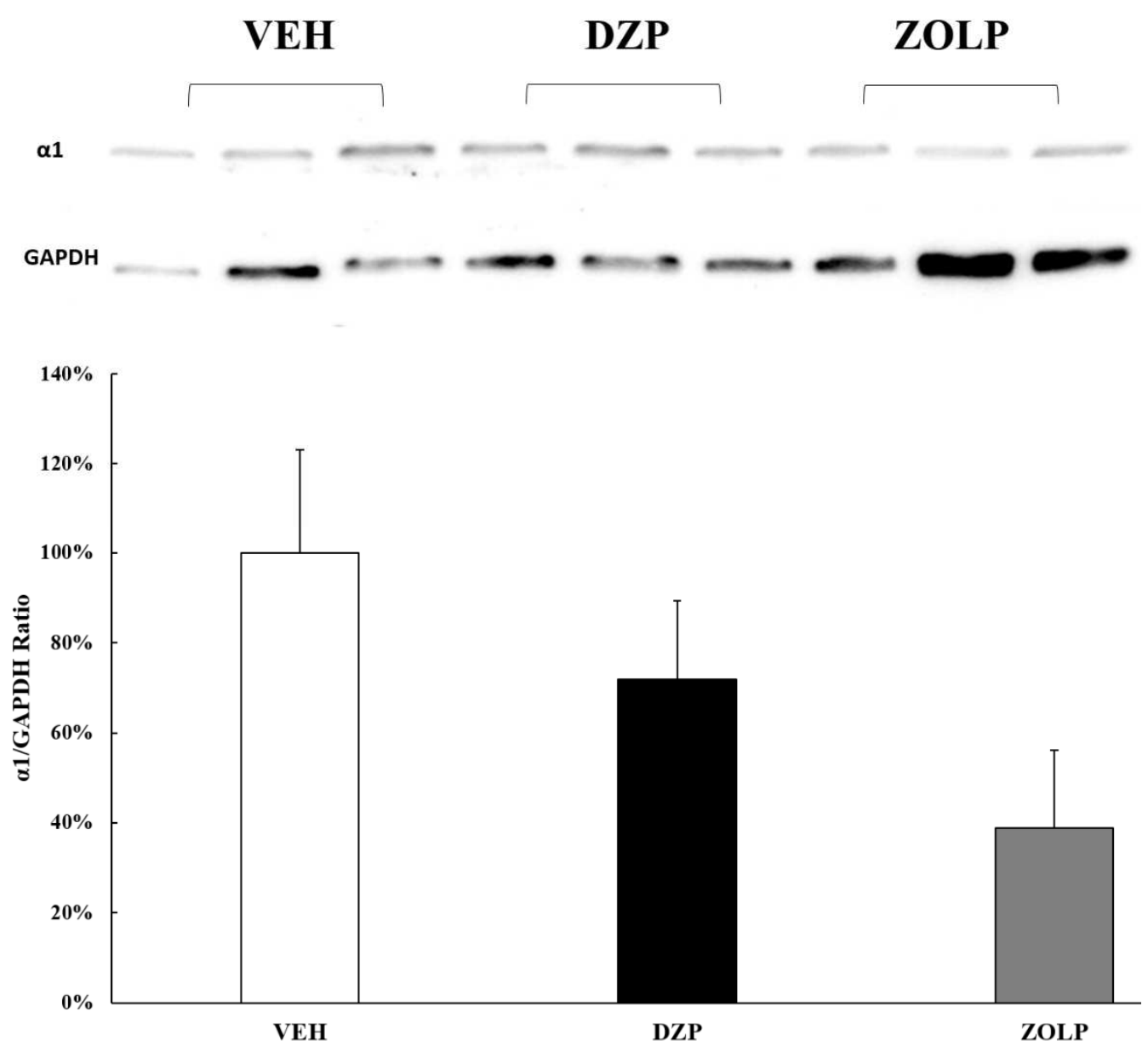

Figure 4-19. Relative $\alpha 1$ subunit expression in the cortex following 7-day administration of zolpidem, diazepam, or vehicle

Relative expression of protein from each group. Histograms comparing levels of transcripts are expressed as mean percentage of VEH control (100\%). Statistical analyses were performed by ANOVA with Dunnett's t test for pairwise comparisons against a VEH. Data are presented as mean + sem. There were no significant differences between groups, $p \mathrm{~s}>0.05$. 


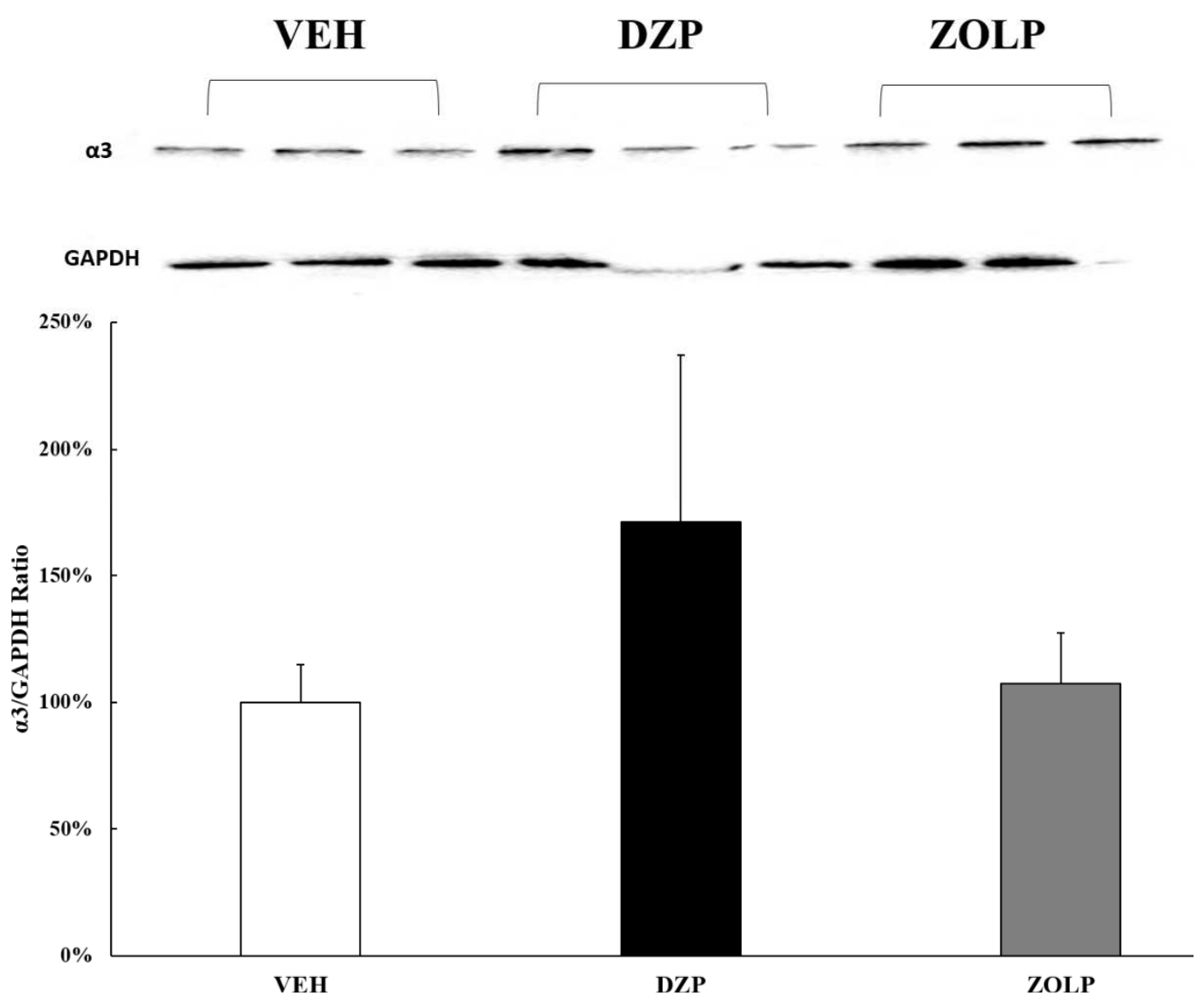

Figure 4-20. Relative $\alpha 3$ subunit expression in the cortex following 7-day administration of zolpidem, diazepam, or vehicle Relative expression of protein from each group. Histograms comparing levels of transcripts are expressed as mean percentage of VEH control (100\%). Statistical analyses were performed by ANOVA with Dunnett's t test for pairwise comparisons against a VEH. Data are presented as mean + sem. There were no significant differences between groups, $p \mathrm{~s}>0.05$. 


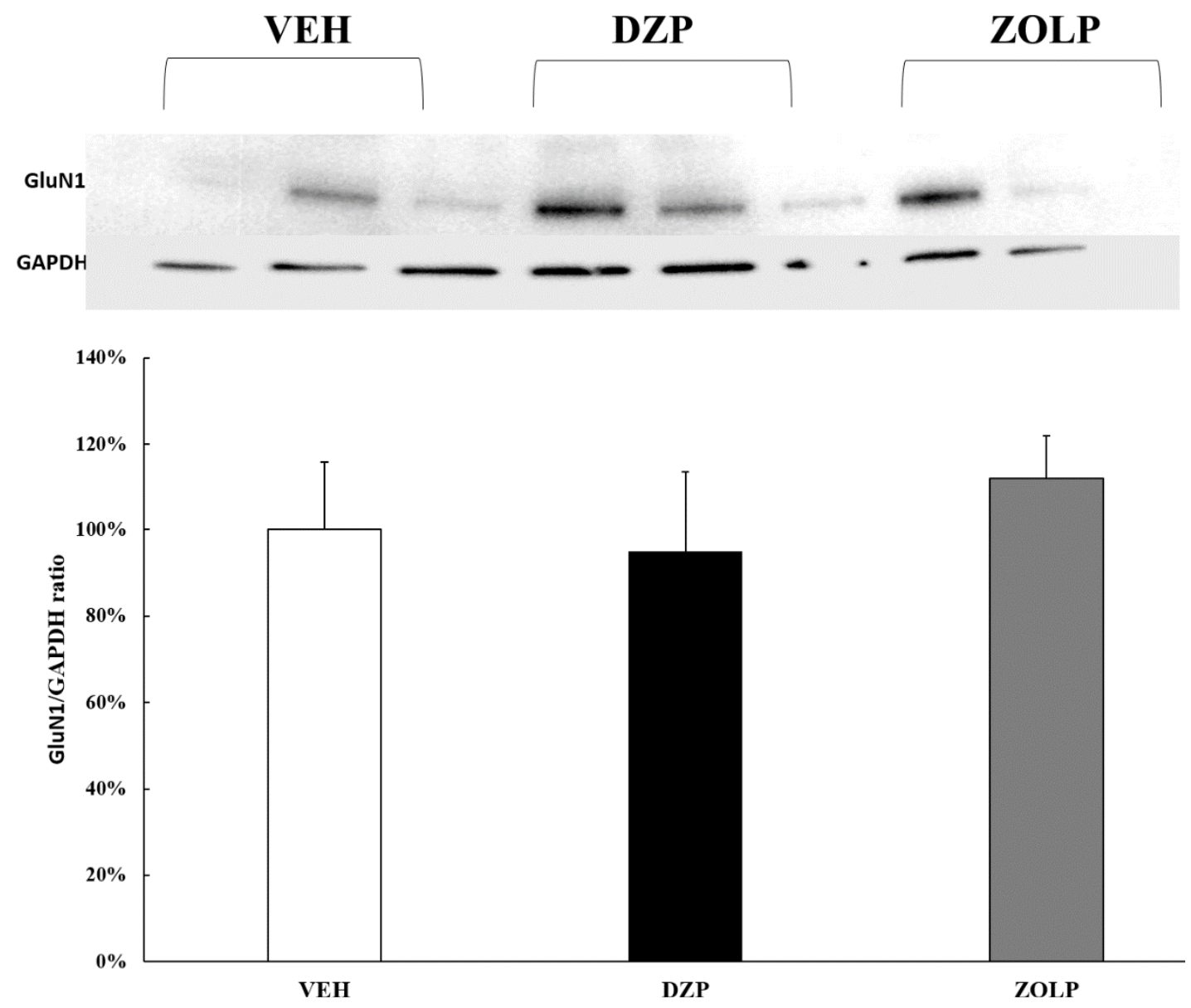

Figure 4-21. Relative GluN1 subunit expression in the cortex following 7-day administration of zolpidem, diazepam, or vehicle

Relative expression of protein from each group. Histograms comparing levels of transcripts are expressed as mean percentage of VEH control (100\%). Statistical analyses were performed by ANOVA with Dunnett's t test for pairwise comparisons against a VEH. Data are presented as mean + sem. There were no significant differences between groups, $p \mathrm{~s}>0.05$. 


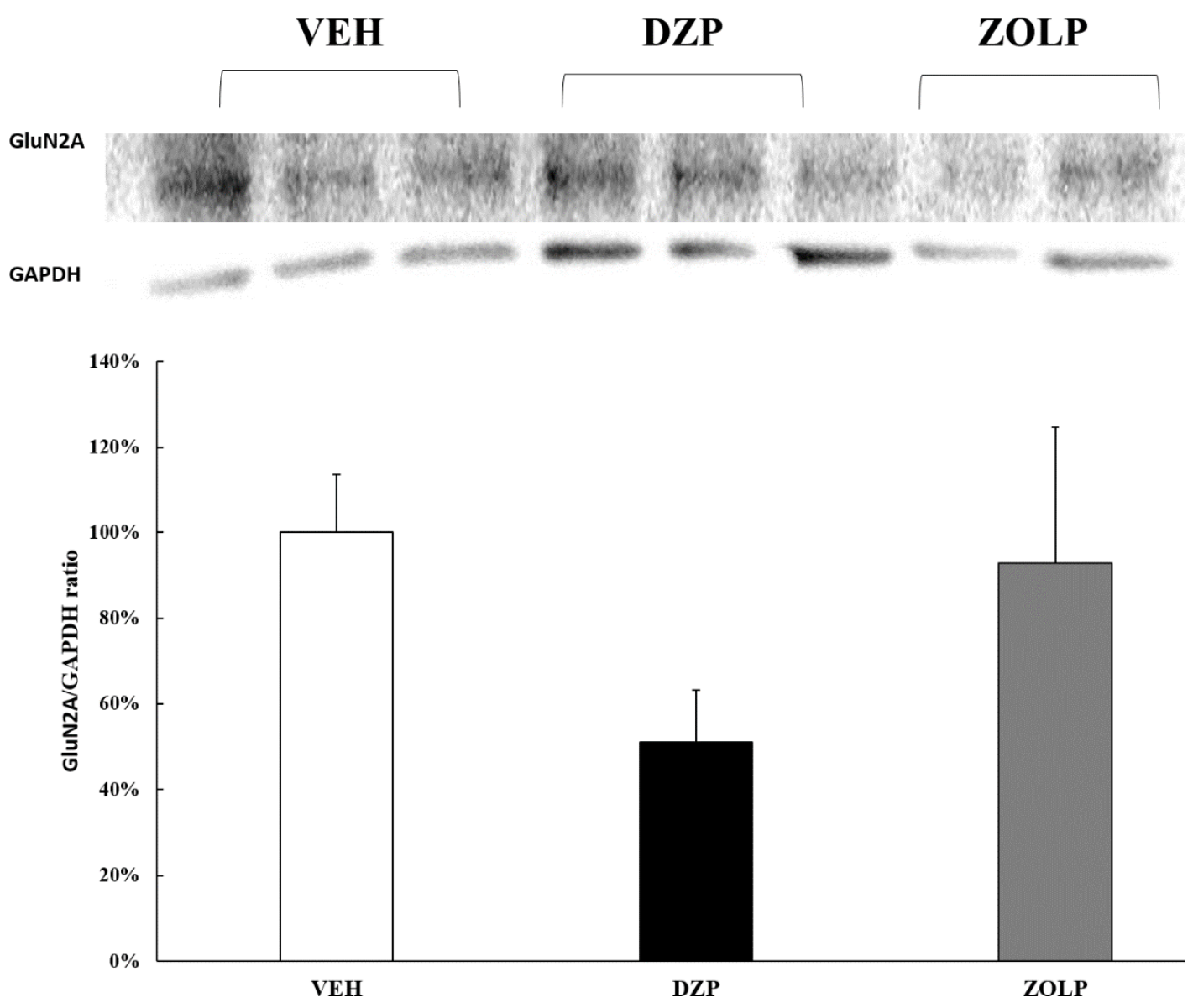

Figure 4-22. Relative GluN2A subunit expression in the cortex following 7-day administration of zolpidem, diazepam, or vehicle

Relative expression of protein from each group. Histograms comparing levels of transcripts are expressed as mean percentage of VEH control (100\%). Statistical analyses were performed by ANOVA with Dunnett's t test for pairwise comparisons against a VEH. Data are presented as mean + sem. There were no significant differences between groups, $p \mathrm{~s}>0.05$. 

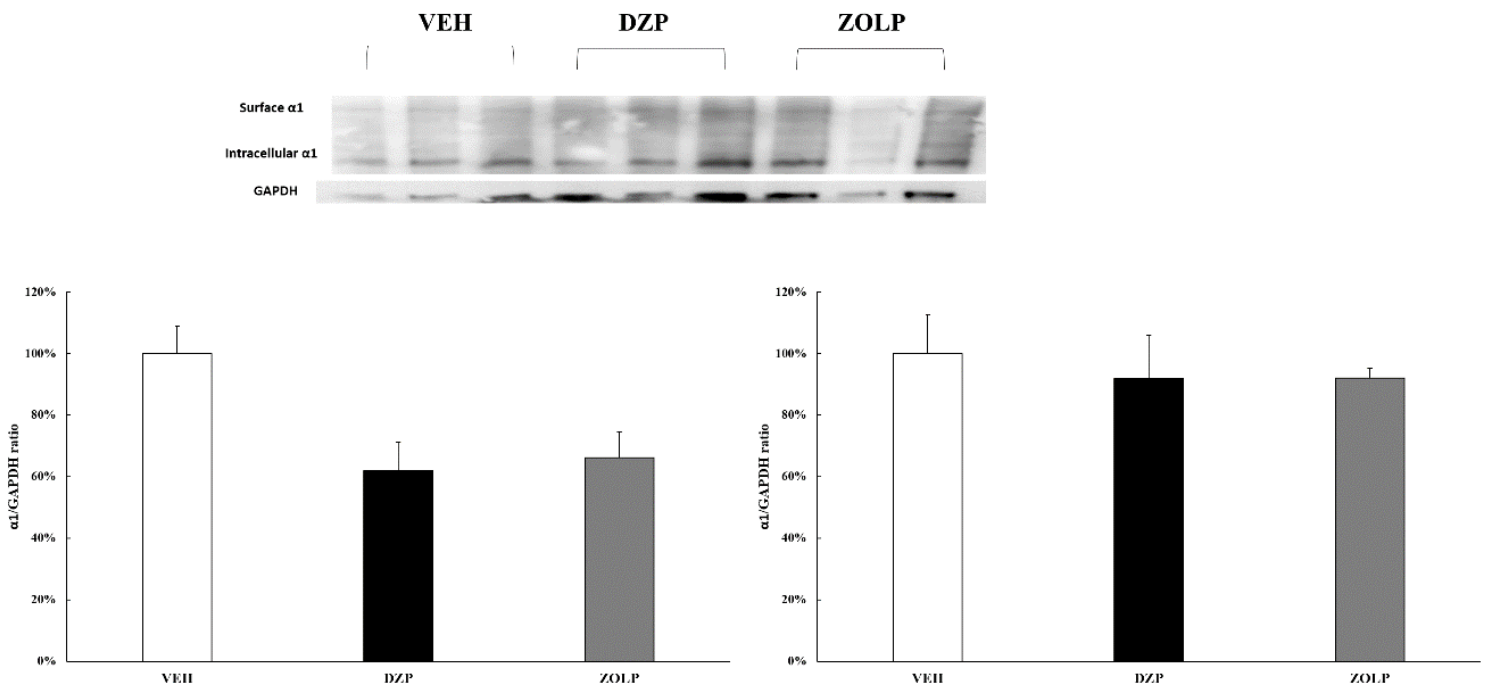

Figure 4-23. BS3 treated samples, surface and intracellular $\alpha 1$ protein expression levels in the cortex following 7-day administration of zolpidem, diazepam, or vehicle Relative expression of protein from each group. Histograms comparing levels of transcripts are expressed as mean percentage of VEH control (100\%). Statistical analyses were performed by ANOVA with Dunnett's $t$ test for pairwise comparisons against a VEH. Data are presented as mean + sem. There were no significant differences between groups, $p \mathrm{~s}>0.05$. 


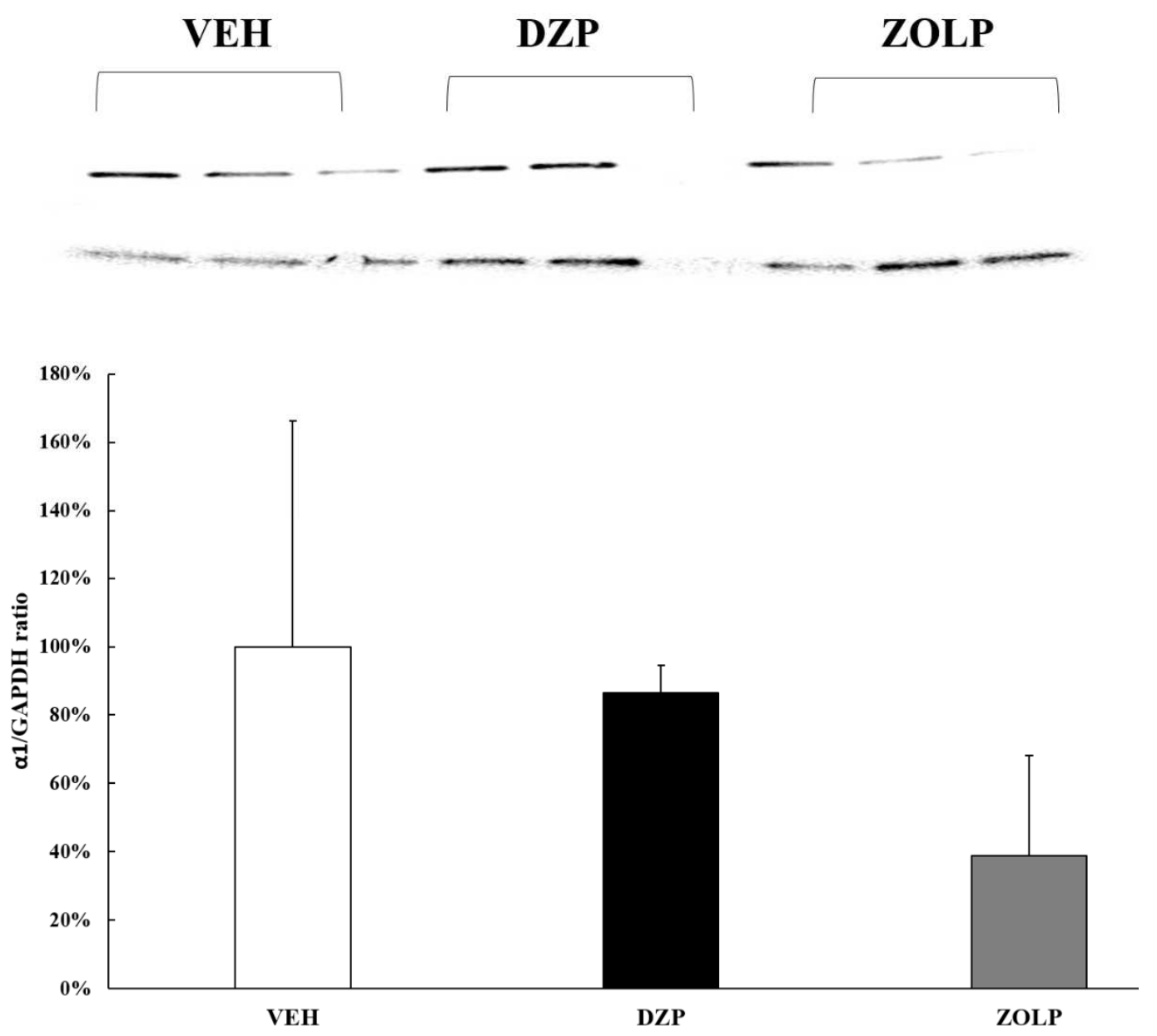

Figure 4-24. Relative $\alpha 1$ protein expression levels in the hippocampus following 7day administration of zolpidem, diazepam, or vehicle Relative expression of protein from each group. Histograms comparing levels of transcripts are expressed as mean percentage of VEH control (100\%). Statistical analyses were performed by ANOVA with Dunnett's t test for pairwise comparisons against a VEH. Data are presented as mean + sem. There were no significant differences between groups, $p \mathrm{~s}>0.05$. 


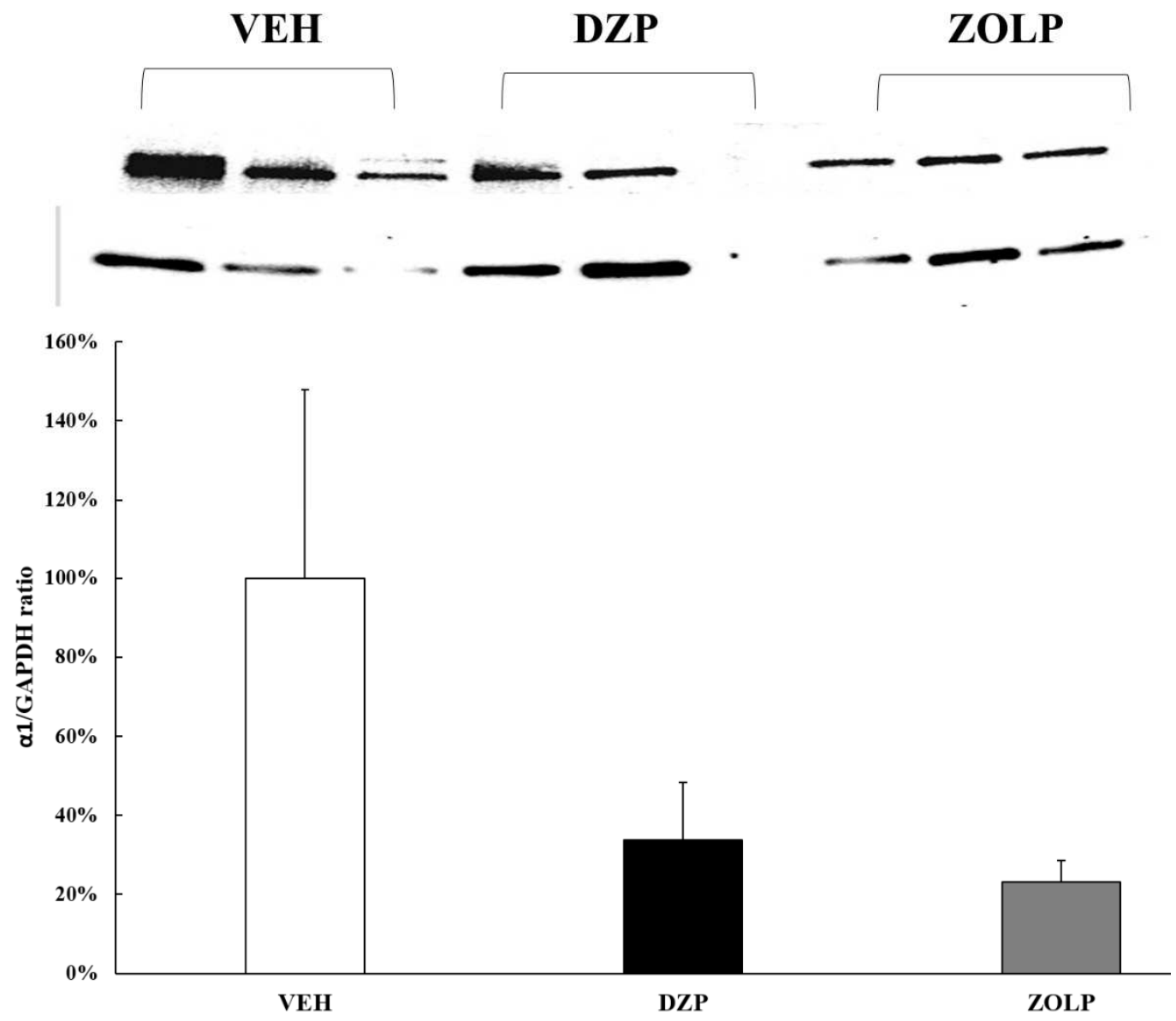

Figure 4-25. Relative $\alpha 2$ protein expression levels in the hippocampus following 7day administration of zolpidem, diazepam, or vehicle

Relative expression of protein from each group. Histograms comparing levels of transcripts are expressed as mean percentage of VEH control $(100 \%)$. Statistical analyses were performed by ANOVA with Dunnett's t test for pairwise comparisons against a VEH. Data are presented as mean + sem. There were no significant differences between groups, $p \mathrm{~s}>0.05$. 
behaviors, including decreases in activity, frequency of rearing, and center zone entries. These mice also showed a progressive decrease percent of time in center zone, suggesting a gradual increase in anxiety during testing. In contrast, zolpidem and diazepam treated mice showed minimal withdrawal symptoms after flumazenil treatment, as indicated by the lack of anxiety-like behaviors when flumazenil was given prior to testing 1 and 2 days after drug termination.

In addition to changes in behaviors, mice given zolpidem or diazepam treatment for 7 days also showed different levels of mRNA expression in the cortex when compared to vehicle mice. Both drugs caused decreases in the expression of $\alpha 1-, \alpha 3-, \beta 2-$, and $\delta-\mathrm{GABA}_{\mathrm{A}} \mathrm{R}$ subunits in the cortex. Diazepam treatment also caused decreases in the expression $\alpha 4-, \alpha 5-, \beta 1-$, and $\gamma 2-G_{A B A} R$ subunits in the cortex. Mice given diazepam treatment also showed different levels of mRNA expression in the hippocampus. Specifically, these mice displayed lower mRNA expression levels of $\alpha 5$ - and $\gamma 2$ $\mathrm{GABA}_{\mathrm{A}} \mathrm{R}$ subunits and the NMDA GluN2A subunit.

Study 2 also identified differences in local protein expression. Protein expression of total $\alpha 2$ subunit was decreased after zolpidem treatment and protein expression of total $\gamma 2$ subunit was decreased after diazepam treatment in the cortex. The intracellular $\alpha 1$ subunit protein was decreased after zolpidem treatment, while surface $\alpha 1$ subunit protein was unaffected in the cortex. Protein expression of total $\alpha 1$ and $\alpha 2$ subunits in the hippocampus were not affected.

\section{Study 3: The Effect of 30-Day Zolpidem and Diazepam Treatment on Behavioral Sedation and mRNA Expression}

\section{Tolerance to the Sedative Effects of Zolpidem}

The purpose of Study 1 was to examine if a 30-day treatment regimen of zolpidem or diazepam induces significant changes to the sedative effects of zolpidem, as measured by open field activity, and significant changes in mRNA expression, as measured by qRT-PCR. Changes to the sedative effects were examined in Cohort 1 and 2 mice previously treated with vehicle, diazepam, and zolpidem. A two-way ANOVA on found a significant main effect of time, $F(5,175)=196.10, p<0.01$, a significant main effect of treatment, $F(2,35)=8.95, p<0.01$, and a significant interaction of Time $\mathrm{x}$ Treatment, $F(10,175)=2.57, p<0.01$ (Figure 4-26). To examine the interaction, oneway ANOVAs were performed at each level of time. There were significant group differences at Block 1, $F(2,54)=4.15, p<0.05$, and Block 2, $F(2,54)=6.57, p<0.01$. There were no significant differences at Blocks 3-6, $F(2,54)<4.14, p>0.05$. Follow-up Dunnett's $t$-tests found that the DZP group displayed more activity than the VEH group at Block $1, p<0.05$. At Block 2, both DZP and ZOLP groups exhibited more activity than the VEH group, $p \mathrm{~s}<0.05$. 


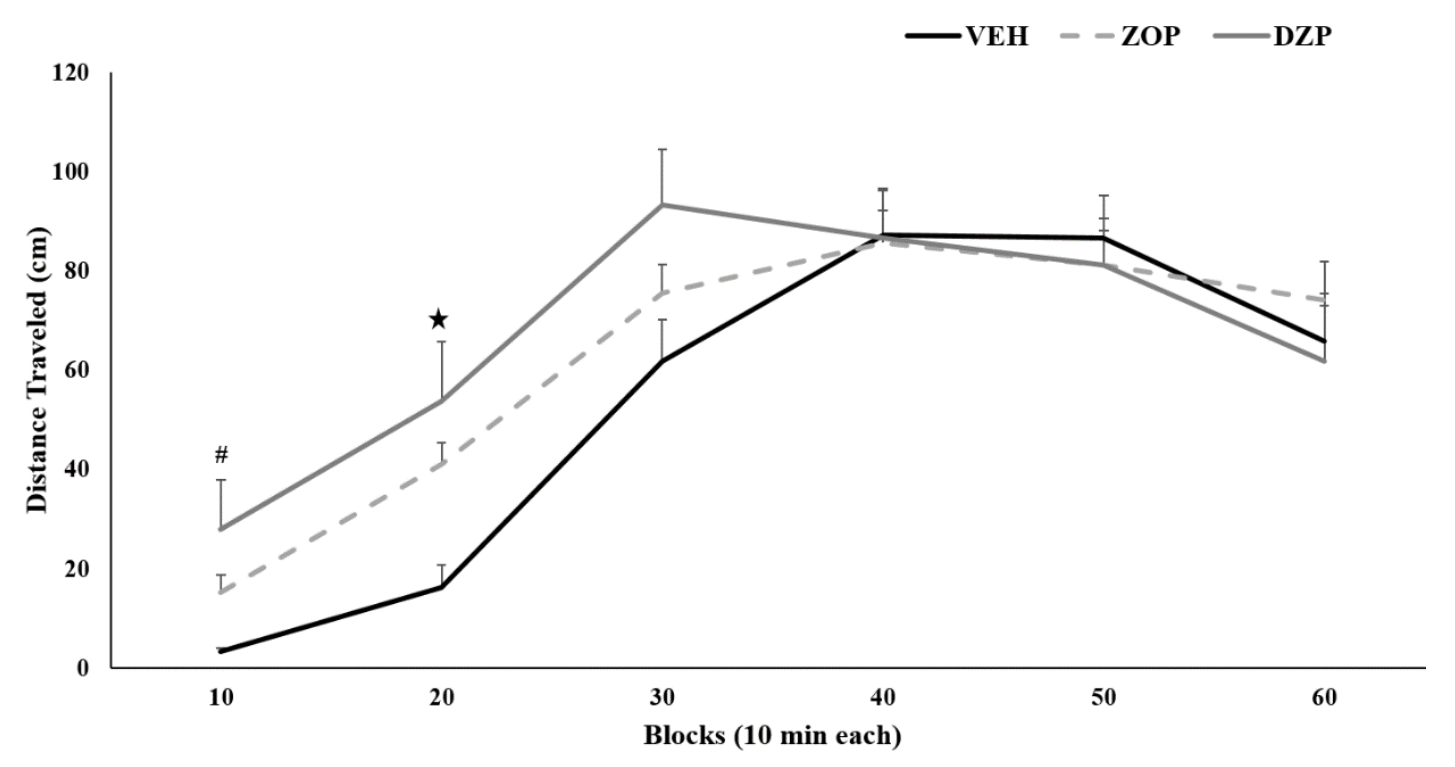

Figure 4-26. Decreased locomotor impairing effect of zolpidem after 30-day administration of zolpidem, diazepam, or vehicle

Distance traveled in open field after test administration of $2 \mathrm{mg} / \mathrm{kg}$ of ZOLP in mice previously given subchronic treatment of ZOLP, DZP, or VEH. Analyses of each 10-min block showed that differences between groups were most evident in the first half of the 60-min test session. Data are presented as mean + sem. Black star denotes VEH $<$ ZOLP and DZP groups and Number sign denotes VEH $<$ DZP group $(p<0.05)$. 


\section{Changes in mRNA Expression Measured by qRT-PCR}

Cohort 2 mice were used to examine changes in mRNA expression. One-way ANOVAs were performed to compare group levels of each mRNA in the cortex, hippocampus, PFC, and amygdala. In the cortex, one-way ANOVAs revealed significant group differences in mRNA levels of $\alpha 1$ subunits, $F(2,35)=5.18, p<0.05$, and GAT-1, $F(2,35)=3.30, p<0.05$. In the hippocampus, ANOVAs revealed significant group differences in levels of $\alpha 4$ subunits, $F(2,31)=5.89, p<0.01$, $\gamma 2$ subunits, $F(2,31)=3.60$, $p<0.05$, and gephyrin, $F(2,31)=4.39, p<0.05$. In the amygdala, a one-way ANOVAs revealed significant group differences in mRNA levels of $\gamma 2$ subunits, $F(2,18)=6.84, p<$ 0.01. Follow-up Dunnett's $t$-tests performed on all group differences above show that DZP or ZOLP groups were not significantly different from the VEH groups, $p \mathrm{~s}>0.05$ (Figure 4-27).

In the PFC, one-way ANOVAs found group differences in mRNA levels of subunits $\alpha 1, F(2,30)=6.67, p<0.01 ; \alpha 2, F(2,27)=6.70, p<0.01 ; \alpha 3, F(2,31)=5.39, p$ $<0.05 ; \beta 1, F(2,32)=4.23, p<0.05 ; \beta 2, F(2,32)=3.75, p<0.05 ; \beta 3, F(2,32)=5.33, p<$ $0.05 ; \delta, F(2,32)=4.16, p<0.05 ; \gamma 1, F(2,32)=5.90, p<0.01$; and $\gamma 2, F(2,32)=4.07$, $p s$ $<0.05$. Follow-up Dunnett's $t$-test found significant differences between the VEH and ZOLP groups for subunits $\alpha 1, \alpha 2, \alpha 3, \beta 1, \beta 2, \beta 3, \delta, \gamma 1$, and $\gamma 2, p s<0.05$. (Figure 4-28).

In summary, this study indicated that mice given a 30-day treatment of diazepam were cross-tolerant to the sedative effects of zolpidem. This study also indicated that mice given zolpidem showed some evidence of zolpidem tolerance; however, this effect was relatively weak and only observed at time Block 2 . There were no significant changes in mRNA levels after 30 days of diazepam. However, mice given a 30-day zolpidem treatment did significantly decrease the levels of mRNA for GABAAR subunits $\alpha 1, \alpha 2, \alpha 3, \beta 1, \beta 2, \beta 3, \gamma 1$, and $\gamma 2$ in the PFC.

\section{Comparisons of Zolpidem and Diazepam Sedative Effects after 3-, 7-, and 30-Day Treatment Durations}

Statistical analyses of Studies 1-3 revealed more prominent deficits to the sedative effects of zolpidem in mice given zolpidem and diazepam for 7 days in comparison to 3 days and 30 days. To further inspect these findings, we compared differences in treatment duration by drug treatment. To complete this, we first examined whether differences in treatment duration (3-, 7-, and 30-days) influenced the distance traveled across the test session time (Blocks 1-6) in mice given vehicle treatments (VEH-ZOLP). This comparison indicated no significant of time, $F(5,240)=98.09, p<0.01$; duration, $F(10,240)=1.35, p>0.05$; or a significant Time $\mathrm{x}$ Duration interaction, $F(2,48)=0.90$, $p>0.05$. These results suggest that the number of injections prior to testing did not impact locomotor activity during testing and was not likely a confounding factor influencing differences in the sedative effects of zolpidem among studies (Figure 4-29). 


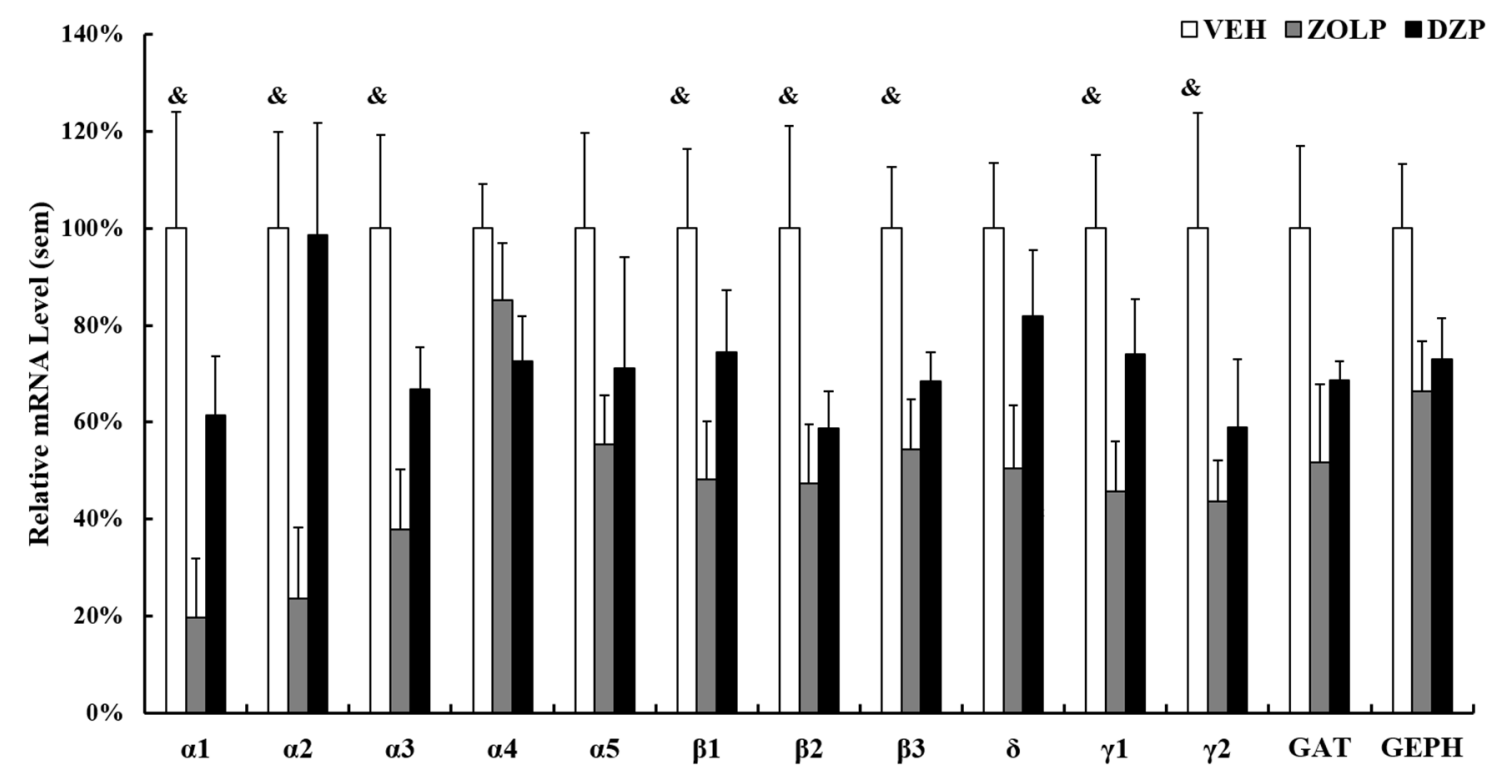

Figure 4-27. Relative levels of mRNA in the pfc following 30-day administration of zolpidem, diazepam, or vehicle

Histograms comparing levels of transcripts are expressed as mean percentage of VEH control (100\%). Statistical analyses were performed by ANOVA with Dunnett's $t$ test for pairwise comparisons against a VEH. Data are presented as mean + sem. Ampersand sign $\mathrm{VEH}>$ ZOLP treatment $(p<0.05)$. 

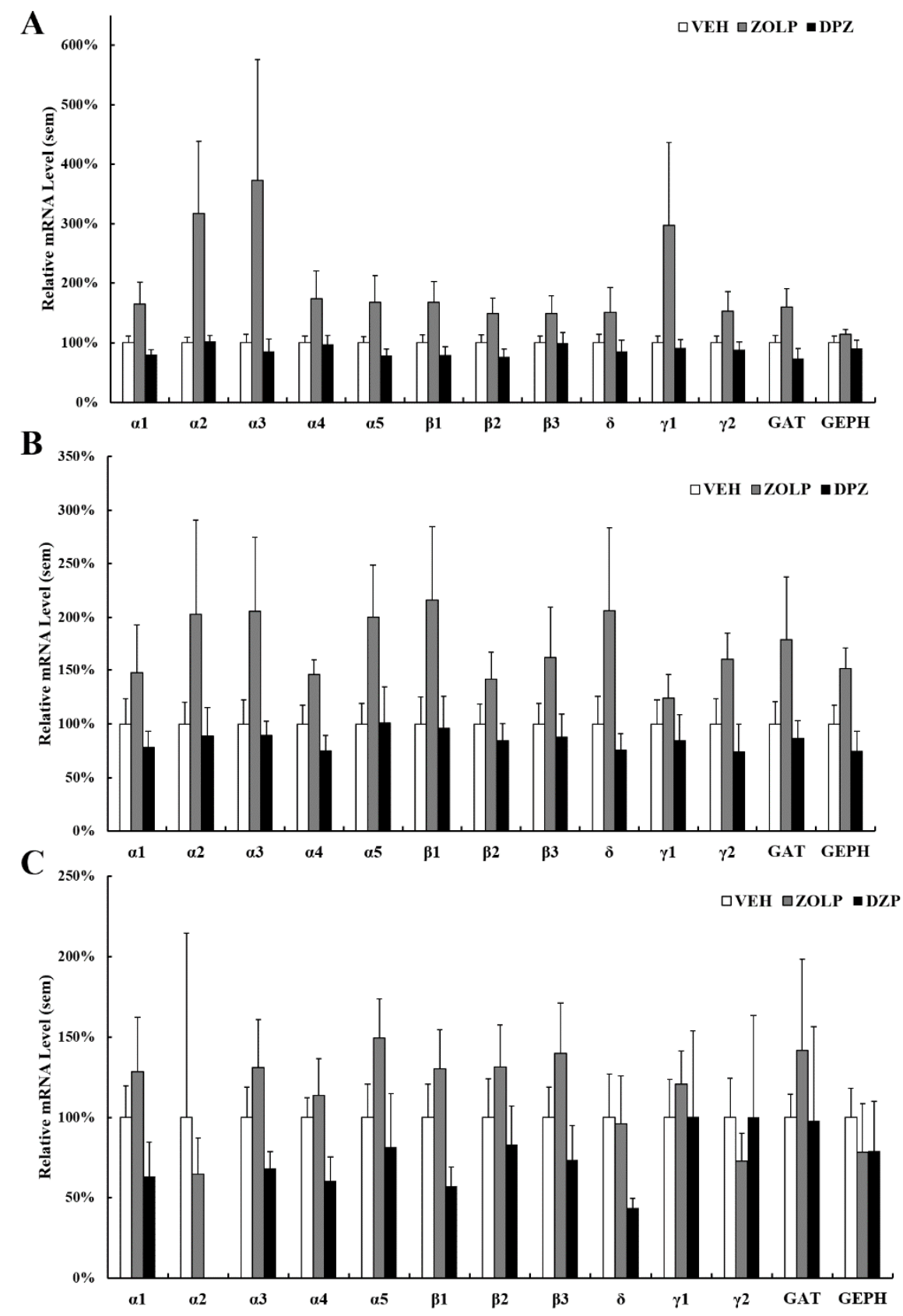

Figure 4-28. Relative levels of mRNA in the cortex, hippocampus, and amygdala following 7-day administration of zolpidem, diazepam, or vehicle

Histograms comparing levels of transcripts are expressed as mean percentage of VEH control $(100 \%)$. Statistical analyses were performed by ANOVA with Dunnett's $t$ test for pairwise comparisons against a VEH. Data are presented as mean + sem. There were no significant differences between groups, $p \mathrm{~s}>0.05$. 


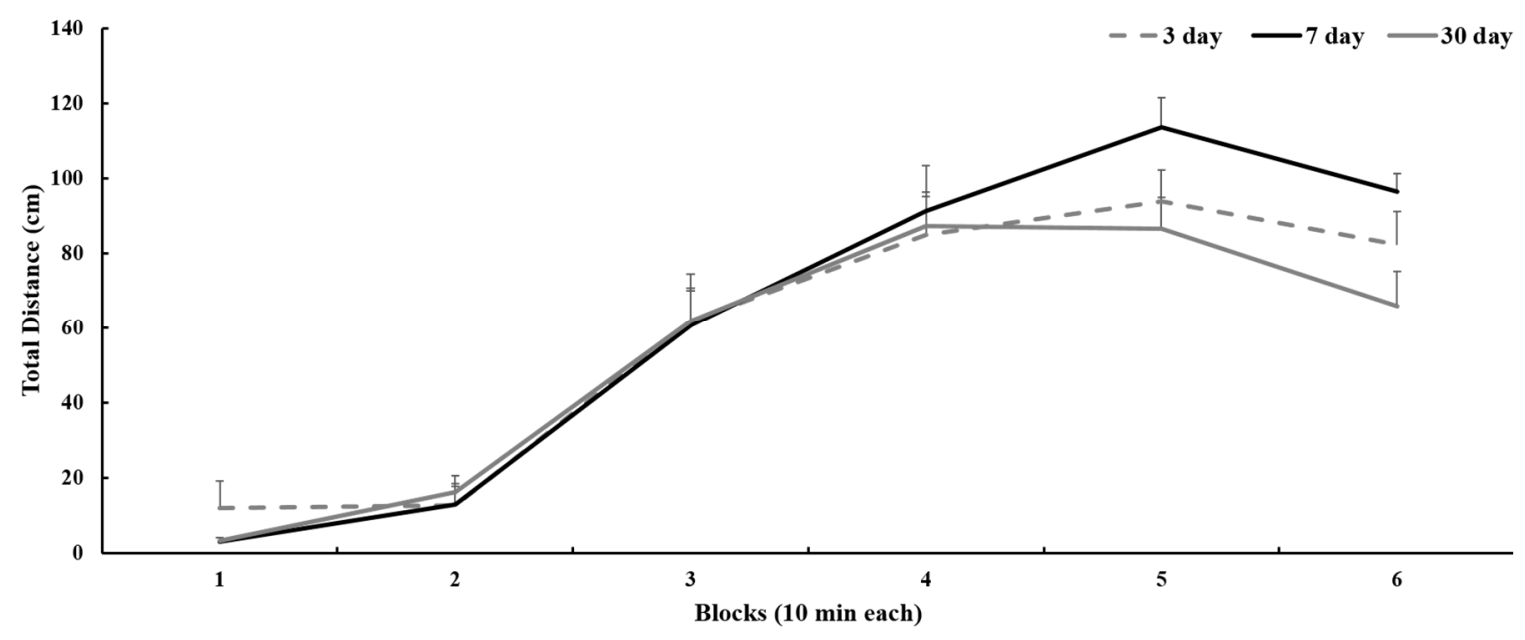

Figure 4-29. No significant differences between vehicle groups from each study on motor activity

Distance traveled in open field after test administration of $2 \mathrm{mg} / \mathrm{kg}$ of ZOLP in mice previously given subchronic treatment of VEH for 3, 7, or 30 days. Analyses of each 10min block showed no differences between. Data are presented as mean + sem. 
Next, we used the same approach in an attempt to affirm the more prominent 7day deficits by separately examining differences in treatment durations of zolpidem and diazepam. In mice given diazepam treatments (DZP-ZOLP), the two-way ANOVA revealed a main effect of time, $F(5,240)=52.20, p<0.01$. There was no effect of duration, $F(2,48)=1.60, p>0.05$ or a significant interaction of Time $\mathrm{x}$ Duration, $F(10$, $240)=1.43, p>0.05$ (Figure 4-30).

In mice given zolpidem treatments (ZOLP-ZOLP), the two-way ANOVA found a main effect of time, $F(5,295)=105.08, p<0.01$, duration, $F(2,59)=6.72, p<0.05$, and a significant interaction of Time $\mathrm{x}$ Duration, $F(10,295)=1.92, p<0.05$. To evaluate the interaction, one-way ANOVAs were conducted on each block of time. There was a significant difference between groups at Block $2, F(2,59)=3.98, p<0.05$, Block 3 , $F(2,59)=9.18, p<0.05$, and Block $6, F(2,59)=3.85, p<0.05$. Mice who received zolpidem for 7 days displayed greater activity at Blocks 2, 3, and 6 than mice who received zolpidem for 3 or 30 days, $p<0.05$. There were no significant differences between groups at Blocks 1, 4 and $5, F_{\mathrm{s}}(2,59)<3.10, p \mathrm{~s}>0.05$ (Figure 4-31).

\section{Comparison of Center, and Periphery Distance Traveled: Spontaneous Withdrawal}

To assess whether differences in the distance traveled across the test session time (Blocks 1-6) influenced measures of open-field anxiety, two-way ANOVAs were performed on distance traveled in the center and periphery of the open field. If both regions show decreases in activity, then activity would affect interpretation of anxietylike measures.

For distance traveled in the center, a two-way ANOVA found that there was a main effect of time, $F(5,105)=31.01, p<0.01$, a main effect of treatment, $F(2,21)=$ $7.21, p<0.01$, and a significant interaction of Time $\mathrm{x}$ Duration, $F(10,105)=3.24, p<$ 0.01 . To assess the interaction, a one-way ANOVAs at each level of time were performed. There were group differences at Block 1, $F(2,21)=14.82, p<0.01$, Block 2, $F(2,21)=9.09, p<0.01$, Block 3, $F(2,21)=4.05, p<0.05$, and Block 5, $F(2,21)=3.51$, $p<0.05$. Follow-up Dunett's $t$-tests revealed that the activity of ZOLP and DZP groups was significantly lower than the $\mathrm{VEH}$ groups at Blocks 1 and 2, $p<0.01$ and the activity of DZP groups was significantly lower than the VEH groups at Blocks 3 and $5, p<0.05$. There was no significantly differences at Blocks 4 and $6, F \mathrm{~s}(2,21)>3.17, p>0.05$

(Figure 4-32).

For distance traveled in the periphery, a two-way ANOVA found that there was a main effect of time, $F(5,105)=11.36, p<0.05$, no main effect of treatment, $F(2,21)=$ 2.03, $p>0.05$, and no significant interaction of Time $\mathrm{x}$ Duration, $F(10,105)=0.81, p>$ 0.05 (Figure 4-33). 


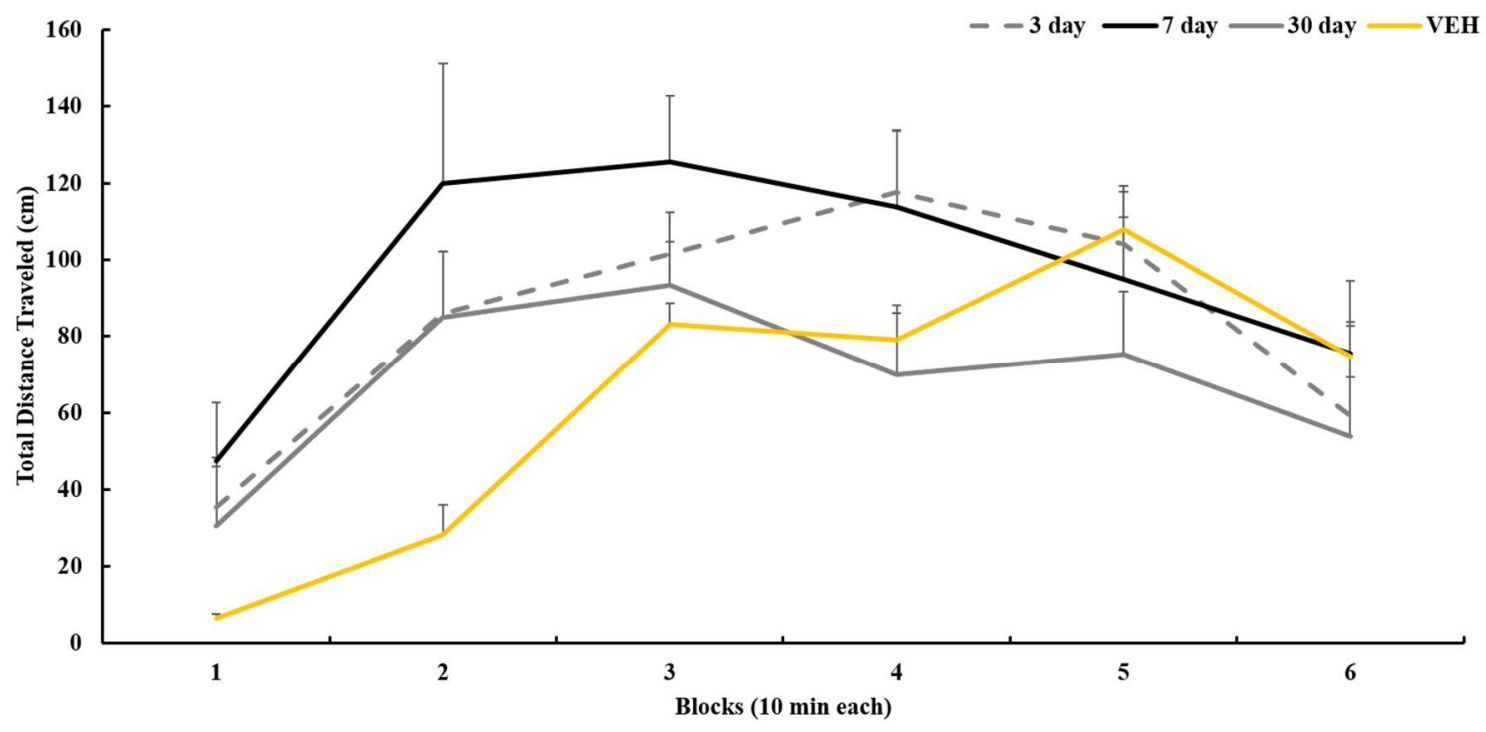

Figure 4-30. No significant differences between diazepam groups from each study on motor activity.

Distance traveled in open field after test administration of $2 \mathrm{mg} / \mathrm{kg}$ of ZOLP in mice previously given subchronic treatment of DZP for 3, 7, or 30 days. Analyses of each 10min block showed no differences between. Data are presented as mean + sem. 


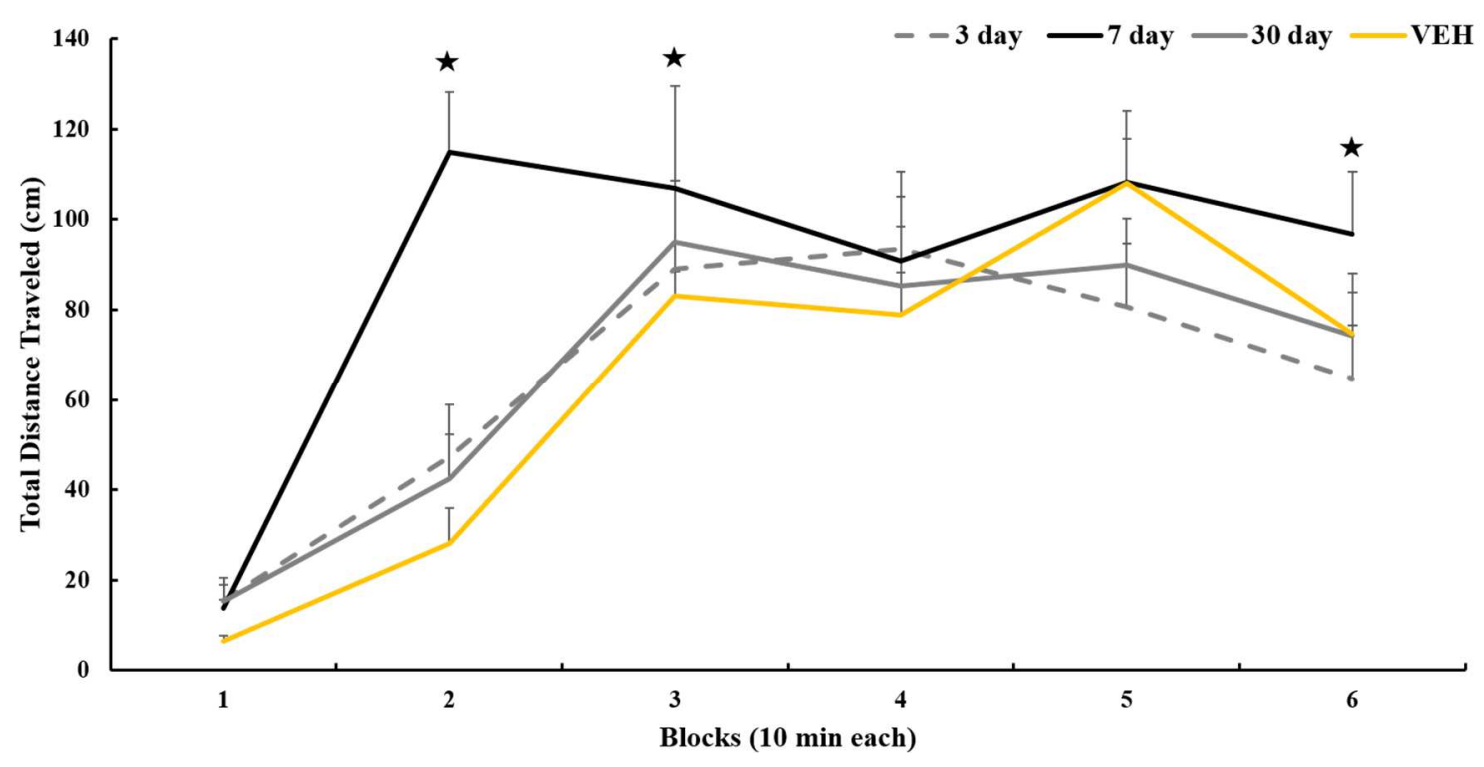

Figure 4-31. Increased motor activity due to 7 days of ZOLP compared to 3 or 30 days of ZOLP

Distance traveled in open field after test administration of $2 \mathrm{mg} / \mathrm{kg}$ of ZOLP in mice previously given subchronic treatment of ZOLP for 3, 7, or 30 days. Analyses of each 10min block showed differences between groups. The 7 day ZOLP group showed greater activity than the 3 or 30 -day group. Data are presented as mean + sem. 


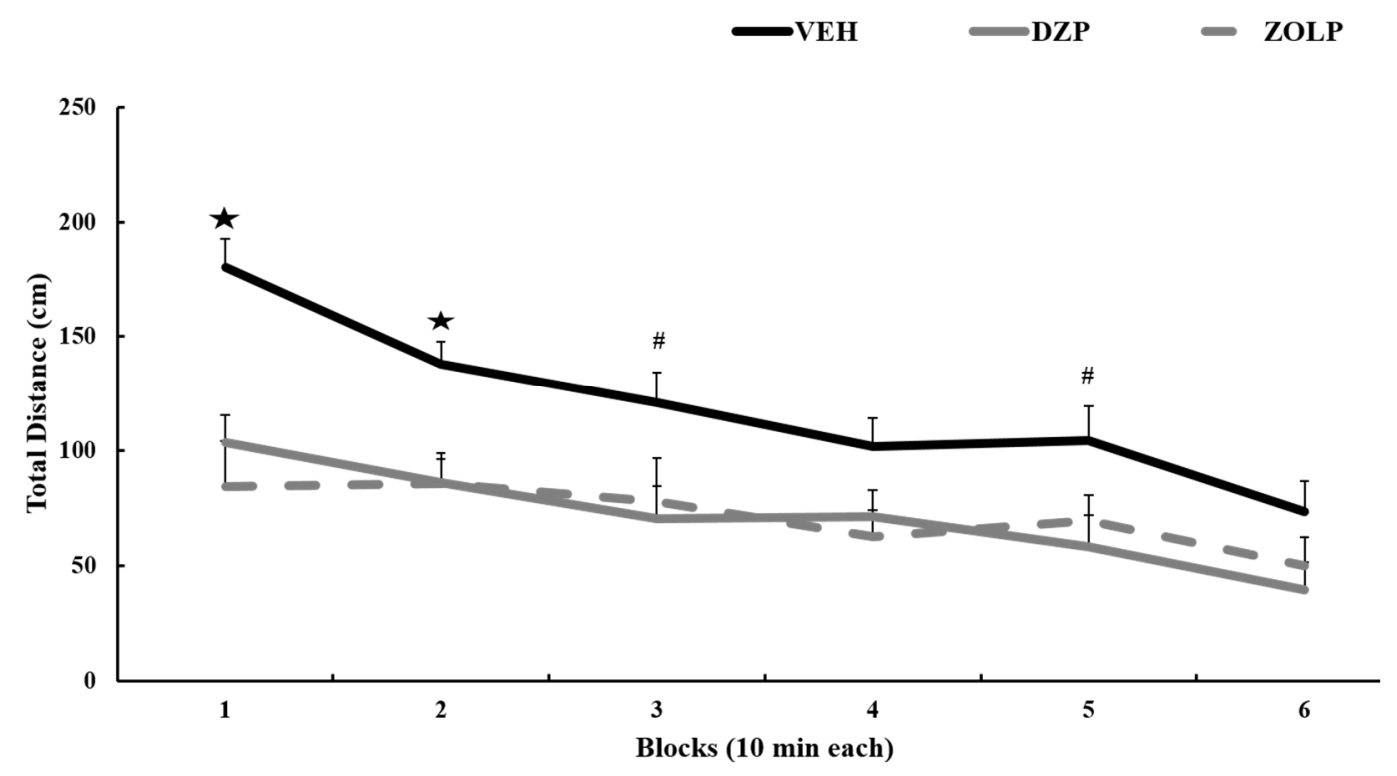

Figure 4-32. Decreased motor activity in the center region of the open field apparatus

Distance traveled in open field after administration of VEH in mice previously given subchronic treatment of ZOLP, DZP, or VEH. Analyses of each 10-min block showed that differences between groups, with the VEH group showing greater activity than the DZP or ZOLP groups. Data are presented as mean + sem. Black star denotes VEH $<$ ZOLP and DZP groups and Number sign denotes VEH $<$ DZP group $(p<0.05)$. 
-VEH DZP $\quad-$ ZOLP

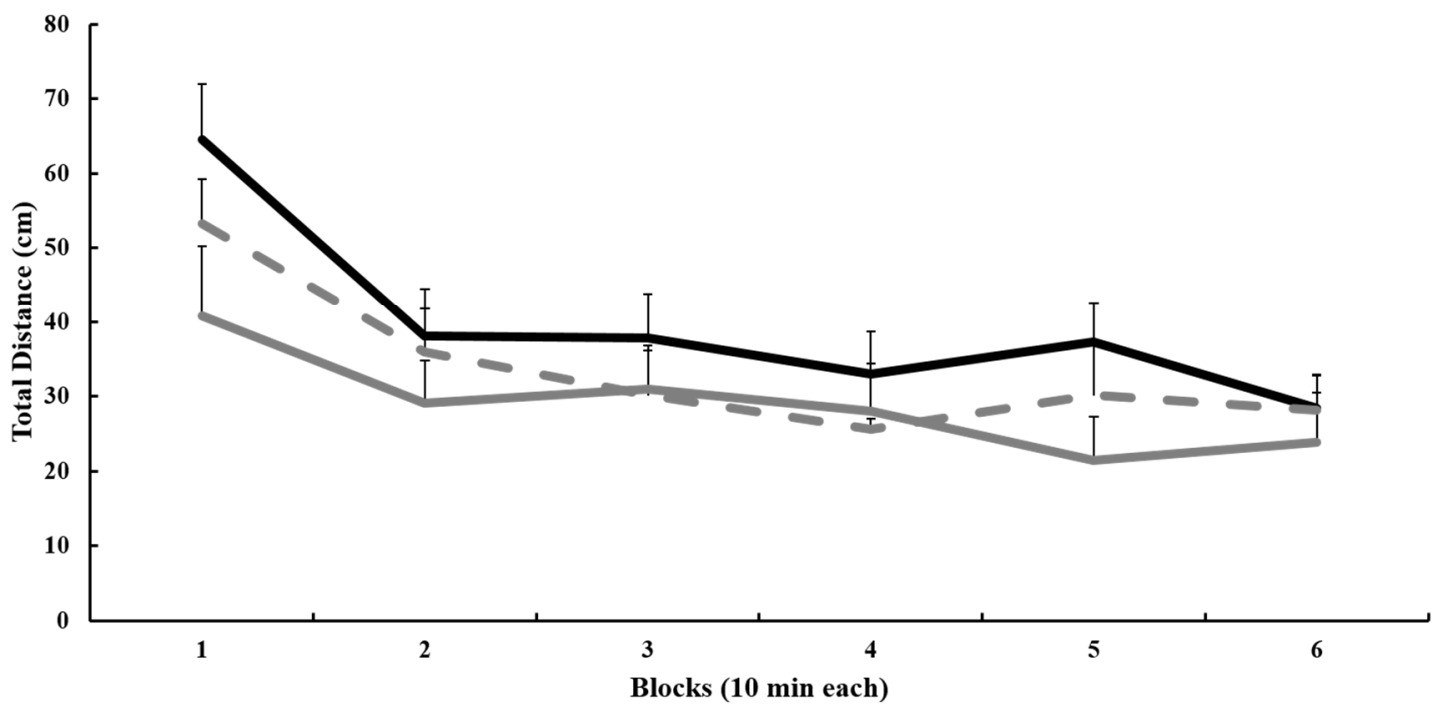

Figure 4-33. No significant differences between groups in the peripheral region of the open field apparatus on motor activity

Distance traveled in open field after administration of VEH in mice previously given subchronic treatment of ZOLP, DZP, or VEH. Analyses of each 10-min block showed no differences between groups, Data are presented as mean + sem. 


\section{CHAPTER 5. DISCUSSION*}

The development of tolerance to the sedative-hypnotic effects of zolpidem and symptoms of withdrawal are becoming a growing concern as usage increases in the general population $[22,23,136]$. Using mouse models, researchers are discerning what factors influence tolerance and withdrawal and the neuromechanisms associated with these phenomena [226]. The purpose of our studies were to examine whether 3-, 7-, and/or 30-day treatment regimens of zolpidem or diazepam diminished the sedative effects of zolpidem in mice. In addition, these studies examined whether treatment regimens changed the expression of mRNA and proteins believed to be associated with tolerance and withdrawal.

\section{Behavioral Tolerance and Cross-Tolerance}

Numerous studies have indicated that tolerance to the sedative effects of BZs can develop following repeated treatments in rodents; however, there is conflicting evidence concerning the development of tolerance to zolpidem [123, 243, 244]. Our finding of tolerance after 7 days of zolpidem is in agreement with past studies that show mice develop sedative tolerance after 10 days of treatment [25, 105, 123]. However, in Studies 1 and 3 , mice given zolpidem for 3 or 30 days prior to testing show minimal evidence of reduced sedation. Past studies examining the development of tolerance to zolpidem's varying effects have primarily examined changes in efficacy or potency after 7-14 days of treatment. For example, tolerance to zolpidem's ataxic, hypothermic, and anticonvulsant effects have been observed in rodents and primates during these time periods $[25,29,38,105,138]$. Other studies have reported no tolerance after zolpidem administration in rodents $[28,109]$. The detection of zolpidem tolerance in past studies is likely influenced by numerous factors, like the time between test injection and behavioral testing. One major difference between our and previous studies was the latency between our test-drug injection and the onset of tolerance testing. In most studies, the time between injections and the start of testing is $15-30 \mathrm{~min}$. Because zolpidem is relatively short acting and quickly metabolized, 15-30 min delay between drug injection and testing may hamper detection of tolerance. In our studies, the time between the test injection and testing was only 2 min. As seen in Figure 4-3, the sedative effects of zolpidem are clearly evident during the first 30 min following this test-drug injection and less evident during the latter half of the test session.

\footnotetext{
*Modified with permission. Wright, B.T., Gluszek, C.F. and Heldt, S.A. The effects of repeated zolpidem treatment on tolerance, withdrawal-like symptoms, and GABAA receptor $m R N A$ s profile expression in mice: comparison with diazepam. Psychopharmacology (Berl), 2014. 231(15): p. 2967-79. and Fitzgerald, A.C., Wright, B.T., and Heldt, S.A. The behavioral pharmacology of zolpidem: evidence for the functional significance of $\alpha 1$-containing GABAA receptors. Psychopharmacology (Berl), 2014. 231(9): p. 1865-96 [16, 245].
} 
In contrast to 7 days, 3 and 30 days of zolpidem treatment produced little evidence of sedative tolerance in mice. The lack of tolerance after 3 days may be due to the fact that sedative tolerance gradually develops and reaches detectable levels only at later time points (e.g. 7 days). It is possible that higher treatment dose levels, different dose regimens, or higher testing dose levels may result in more apparent detection of sedative tolerance. Several experiments can answer this question by increasing the dose to $>5 \mathrm{mg} / \mathrm{kg}$ or incrementally increasing the dose across time [211]. It is also possible that a difference in treatment regime (i.e. subcutaneous or oral treatments) may cause tolerance detection at earlier or later time points [123]. This experiment could possibly lead to different behavioral manifestations of tolerance. It is unclear if the rates of tolerance to zolpidem's effects develop at differently.

In Studies 1-3, we found repeated diazepam treatments diminished the sedative effects of zolpidem, a phenomena termed cross-tolerance. In the case of tolerance, the sedative effects of BZs develop before the other effects (e.g. anxiolytic or anticonvulsant) $[127,132,246]$. In a recent study, Vinkers et al. showed that mice continually administered diazepam for 28 days displayed tolerance to diazepam's sedative effects as well as its hypothermic and anxiolytic effects [112]. While numerous studies have documented cross-tolerance between different BZs in rodents, relatively few have examined cross-tolerance between zolpidem and BZs [24, 105, 247-249]. The Vinkers study also showed that mice given continual zolpidem treatment for 28 days displayed cross-tolerance diazepam's hypothermic and anxiolytic-like effects but not to diazepam's sedative effects [112].

Possible evidence for different mechanisms mediating BZ and zolpidem tolerance have been found with experiments using point-mutation mice. These mice have a single point mutation in an $\alpha$-subunit protein, allowing for the receptor to be BZ- and zolpideminsensitive. One study found that tolerance to the sedative effects of diazepam in mice lacking BZ sensitive $\alpha 5-\mathrm{GABA}_{\mathrm{A}} \mathrm{Rs}$ are greatly reduced [129]. This suggests continual activation of $\alpha 5-\mathrm{GABA}_{\mathrm{A}} \mathrm{Rs}$ is an important contributing factor to the development of tolerance to diazepam. Zolpidem has very little affinity and efficacy at $\alpha 5-\mathrm{GABA}_{\mathrm{A}} \mathrm{Rs}$; thus, it is unlikely that tolerance to the sedative effects of zolpidem is due to activation of $\alpha 5-G_{A B A}$ Rs. This may be indicative of different mechanisms mediating the development of sedative tolerance to BZs versus zolpidem

Together with our results, these findings suggest that chronic BZ treatments can cause cross-tolerance to zolpidem's sedative effects, whereas chronic zolpidem treatments are more limited in inducing cross-tolerance to the effects of BZs. In addition, this implies that deficits in zolpidem-induced sedative effects are mediated by continued allosteric modulation of $\alpha 1-\mathrm{GABA}_{\mathrm{A}}$ Rs. However, at the doses used in our studies it is likely that $\alpha 2-$ or $\alpha 3-\mathrm{GABA}_{\mathrm{A}} \mathrm{Rs}$ are activated; thus we cannot exclude the possibility of these $\mathrm{GABA}_{\mathrm{A}} \mathrm{Rs}$ mediating tolerance. Whereas deficits in BZ-induced sedative effects may be due to the repeated allosteric activation of other BZ sensitive GABA $\mathrm{R}$ subtypes combined with the $\alpha 1-\mathrm{GABA}_{\mathrm{A}} \mathrm{R}$ subtype. This may indicate that different $\mathrm{GABA}_{\mathrm{A}} \mathrm{R}$ subtypes are associated with $\mathrm{BZ}$ and zolpidem sedative tolerance and sedative effects. 
In addition to tolerance, is it possible that the sedative effects of BZs and zolpidem are mediated by different or a combination of different GABA $\mathrm{A}_{\mathrm{R}}$ ? Another question is if tolerance and/or cross-tolerance persists after BZ treatment ends. It has been found in human case studies that patients who abuse zolpidem had previous benzodiazepine abuse as well [26]. This may be indicative of permanent changes in $\mathrm{GABA}_{\mathrm{A}}$ Rs functioning and it would be valuable to see if rodents exhibit sedative tolerance after drug cessation.

\section{Behavioral Withdrawal}

In humans, the abrupt cessation of prolonged BZ treatments can be followed by unpleasant withdrawal symptoms, which are believed to play a role in BZ abuse and dependence $[4,250]$. The abrupt cessation of supratherapeutic doses of zolpidem can also lead to a BZ-like withdrawal syndrome [139, 147, 251-254]. In nonhuman primates and rodents, repeated BZ administration also produces physical dependence, detected by spontaneous and flumazenil-induced withdrawal symptoms [6, 255-258]. In nonhuman primates, these withdrawal effects are also observed after repeated treatment with zolpidem [133, 150]. Withdrawal-like symptoms are less likely observed in rodents after zolpidem discontinuation in comparison to diazepam [24, 28, 38, 152, 259-261]. The distinctive pharmacological profile observed in rodents and primates may be explained by differences in the distribution of $\alpha 1-\mathrm{GABA}_{\mathrm{A}} \mathrm{R}$ subtypes and/or the binding characteristics of zolpidem. In vitro autoradiography experiments reveal substantial differences in $[3 \mathrm{H}]$ zolpidem binding in the brains of rats and humans [76]. These differences in the binding characteristics of specific brain regions may likely contribute to the distinctive pharmacological effects of zolpidem in rodents and primates.

In our study zolpidem and diazepam treated mice given vehicle prior to testing showed spontaneous withdrawal. These mice displayed anxiety-like with decreases in activity, frequency of rearing, and center entries. There were also progressive decreases in percent time spent in the center zone, indicative of increasing anxiety during testing. The display of anxiety-like behavior from spontaneous withdrawal in our study are in line with rodent studies that have identified anxiety-like behavior in zolpidem treated mice after spontaneous withdrawal $[28,259,260]$. Our results are also consistent with previous studies showing anxiety-like behaviors in the elevated plus maze, open field, and social interaction test when assessed 1-3 days after prolonged diazepam treatments [101, 227, $228,246,255,262]$. Though both groups of mice displayed anxiety-like behavior in the open field, they also showed reduced locomotion. Unlike our findings, hyper-locomotion, a typical symptom of spontaneous withdrawal, is detected after abrupt cessation of diazepam [125]. Possible that testing could produce anxiety-like behaviors concomitant with hyper-locomotion at later time points.

While it is possible that the decreases in activity were associated with the residual drug effects during testing, evidence from previous studies suggest this is not the case. In rats, the $t_{1 / 2}$ from plasma is approximately $30 \mathrm{~min}$ for zolpidem and $70 \mathrm{~min}$ for diazepam and its active main metabolite desmethyldiazepam [33, 34, 37, 263-265]. In the case of 
zolpidem, daily injections do not change $\mathrm{t}_{1 / 2}$ values or result in accumulation in plasma or the brain [35]. Repeated injection of zolpidem for up to 28 days is virtually undetectable 3-4 $\mathrm{h}$ after the last injection, and the degradation of zolpidem does not lead to the formation of metabolites that can accumulate and sustain drug action [31, 35, 266]. Daily diazepam injections do not change $\mathrm{t}_{1 / 2}$ values and most studies but not all reveal no plasma accumulation of diazepam or its metabolite after long-term treatment [124, 233, $265,267,268]$. These data argue against dramatic changes in the pharmacokinetics as the basis for the detection of withdrawal.

In contrast to spontaneous withdrawal, Study 2 showed that zolpidem and diazepam treated mice given flumazenil after treatments displayed minimal flumazenilinduced withdrawal, as measured by anxiety-like behavior. The lack of flumazenilinduced withdrawal in diazepam treated mice is contrary to studies that have found that flumazenil treatment results in anxiety-like behavior and hyper-locomotion [51-53]. Our study is in line with another study that did not find anxiety-like behavior in zolpidem treated mice given flumazenil [38].

Study 2 found that spontaneous withdrawal results in anxiety-like behavior and hypo-locomotion. Both spontaneous and flumazenil induced withdrawal can induce other withdrawal symptoms like handling induced convulsions that could be detected. A possible experiment would be to see if cessation of drug can cause handling induced convulsions could be detected after 3, 7, or 30 days of zolpidem or diazepam, as noted by past studies [269]. In addition, examining spontaneous withdrawal at time points beyond one day may provide information about the duration and severity of withdrawal symptoms. While withdrawal can be observed hours after drug discontinuation, some studies have found that BZ withdrawal may not occur until up to 3 days after drug treatment has stopped. If the spontaneous withdrawal experiment conducted had tested withdrawal days later, there may have been hyper-locomotion. Study 2 focused on anxiety-like behavior and locomotor activity due to flumazenil-induced withdrawal but are there other withdrawal symptoms or tests that could occur like convulsions and since flumazenil resulted in minimal effects is it possible that other drugs like PTZ can better induce withdrawal symptoms [25, 200, 269].

It is hypothesized that zolpidem withdrawal and tolerance are due to long-term supratherapeutic dose activation of the $\alpha 2$ - and $\alpha 3-\mathrm{GABA}_{\mathrm{A}} \mathrm{Rs}$ along with $\alpha 1-\mathrm{GABA}_{\mathrm{A}} \mathrm{Rs}$ [175]. While zolpidem binds to $\alpha 1$-subunits with an affinity that is five- to ten-fold greater than $\alpha 2-$ and $\alpha 3-\mathrm{GABA}_{\mathrm{A}} \mathrm{Rs}$, in vitro studies show the relatively high dose of zolpidem used in this study might be sufficient to activate these lower-affinity GABA $\mathrm{A}_{\mathrm{A}}$ in vivo and contribute to deficits in the locomotor-impairing effects of zolpidem and anxiety-like behaviors $[17,270]$. 


\section{mRNA and Protein Expression}

\section{GABAAR Subunits mRNA and Protein}

Repeated BZ administration produces numerous neuroadaptive changes in specific brain regions; however, it is unknown which neuroadaptive factors predict whether a certain BZ possesses the potential to produce tolerance. In our studies, we chose to examine mRNA changes in the cortex, hippocampus, amygdala, and PFC due to their involvement in the control of locomotor activity, sedation, and withdrawal symptoms as well as documented mRNA alterations that occur due to BZs and zolpidem $[111,113,187,234]$. Many studies suggest changes in $\mathrm{GABA}_{\mathrm{A}} \mathrm{R}$ subunit mRNA contribute to tolerance and withdrawal after long-term BZ treatment regimens $[92,106$, $108,111,113,271,272]$.

In agreement with Study 2, most data suggest repeated diazepam treatments alter $\alpha 1$-subunit mRNA expression in the cerebral cortex, although this is not observed in all studies [105-113]. Other studies have shown that diazepam treatment decreases $\alpha 1$ subunit mRNA in the hippocampus and prefrontal cortex [105, 107]. Also diazepam treated mice showed a decrease in $\alpha 4, \beta 1$, and $\gamma 2$ subunits in the cortex and $\alpha 5-, \gamma 2-$ $\mathrm{GABA}_{\mathrm{A}} \mathrm{R}$ subunits, and GluN2A subunits in the hippocampus. This latter finding is consistent with some reports that find decreases in mRNA expression of $\alpha 5$ subunits $[107,113,116]$. Some studies find that repeated diazepam treatment $(>7$ days) induced significant increases in $\alpha 3, \alpha 4, \alpha 5, \beta 1$, and $\gamma 3$ subunits as well as decreases in $\beta 2$ and $\gamma 2$ subunits $[107,273]$. Others find long term diazepam caused an increase in the $\alpha 1$-subunit mRNA expression in the parietal cortex and ventral pallidum but no change in $\alpha 1$ or $\gamma 2$ in the other areas [273]. Study 2 observed cortical decreases in $\alpha 3$ - and $\delta$-subunit mRNA after both treatments and $\alpha 4$-subunit mRNA after diazepam treatment. It has been found that long-term treatment with diazepam increased $\alpha 3$ and $\alpha 4$ mRNA expression in the cerebral cortex, whereas other studies have found no such effects $[107,111,115,116]$.

In mice treated with zolpidem for 3 days, we observed a decrease in the mRNA expression of the $\alpha 5$ subunit in the hippocampus and in mice treated with zolpidem for 30 days showed a decrease in the mRNA expression of $\alpha 1, \alpha 2, \alpha 3, \beta 1, \beta 2, \beta 3, \gamma 1$, and $\gamma 2$ subunits in the PFC. We observed that zolpidem treated mice showed a decrease in $\alpha 1$, $\alpha 3, \beta 2$, and $\delta$ subunits in the cortex. We also found that an acute dose of either drug did not affect mRNA levels (Appendix). One of the most consistent finding is that long-term zolpidem treatment decreases $\alpha 1$ mRNA in cerebral cortex after 14 days of treatment in rats [234]. An upregulation of $\alpha 4 \mathrm{mRNA}$ in the cortex has also been identified after longterm treatment with zolpidem [234]. No previous studies have noted changes in $\delta$ mRNA after long-term zolpidem exposure in vivo [107, 234].

It must be noted that many studies have shown inconsistent findings that limit the essential or key changes underlying tolerance and withdrawal. Also of note is that rats have been historically used to investigate mRNA changes associated with tolerance and withdrawal after BZ treatments, while mice have been used in only a few studies [271, 
272]. Neuroanatomical and behavioral differences between these two species may contribute to published discrepancies [274-276]. Other inconsistencies are due to differences between treatment regimen and subanatomical regions. For example some studies used infusions pumps or s.c. injections [115]. Others look at specific regions like the CA1 region of the hippocampus, frontoparietal motor cortex, or frontoparietal somatosensory cortex $[107,112]$.

Both $\alpha 4-$ and $\delta$-GABA ${ }_{A}$ Rs are usually found in perisynaptic and extrasynaptic regions of the brain, including the cortex [277, 278]. These receptors are insensitive to $\mathrm{BZs}$ and zolpidem, indicating that changes in $\mathrm{GABA}_{\mathrm{A}} \mathrm{R}$ mRNA levels are not necessarily dependent on direct drug-receptor interaction. In cultured cells, withdrawal from repeated treatment of diazepam and zolpidem has been shown to increase $\alpha 4$-subunit mRNA and decrease $\alpha 1$-subunit mRNA, suggesting that the molecular events that underlie withdrawal may involve a shift from BZ-sensitive to BZ-insensitive $\mathrm{GABA}_{\mathrm{A}} \mathrm{R}$ subtypes $[188,189]$. While zolpidem preferentially binds to $\alpha 1-G A B A_{A} R s$, it also possesses some agonistic action on $\alpha 3-\mathrm{GABA}_{A}$ Rs at high doses which may mediate the decrease in $\alpha 3$ mRNA after zolpidem treatment $[17,279]$. It is noteworthy that the anxiolytic effects of BZs are putatively mediated by $\alpha 2-$ and $\alpha 3-\mathrm{GABA}_{\mathrm{A}} \mathrm{Rs}[99,280,281]$. While no mRNA changes were detected in the amygdala, which is known to be involved in fear and anxiety, changes in the function of these receptors in the cortex may play a role in the processing of sensory information that mediate anxiety-like behavior seen in diazepam and zolpidem groups treated with vehicle in Study 2 [232].

Due to the development of cross-tolerance in diazepam treated mice to zolpidem and the lack of tolerance in zolpidem treated mice in Study 1 and 3, it was expected that the diazepam treated mice would show changes in mRNA expression. Vinkers 2012, found that 28 days of diazepam resulted in an increase in the $\alpha 2$ subunit in the hippocampus (CA1 region) [112]. Though this study showed mRNA changes in the $\alpha 2$ subunit in the hippocampus, like our study it did not show any changes in the cortex or in the hippocampus with $\alpha 1$ or $\alpha 5$ subunit. It has been shown that the mRNA expression of the $\alpha 4$ subunit increases during tolerance and during withdrawal from diazepam and zolpidem [115, 234]. In the present study we did not see a similar increase in either group.

One would expect since BZs like diazepam have similar affinity and efficacy to multiple $\mathrm{GABA}_{\mathrm{A}} \mathrm{Rs}$, that changes in mRNA transcripts would be more pronounced and frequent than zolpidem. This may be the reason we saw more pronounced alterations due to diazepam versus zolpidem in Study 2. An expectation is that zolpidem would only alter $\alpha 1$ subunits because it primarily binds to $\alpha 1$-GABAARs. As our study and other studies have found repeated zolpidem does have more broad effects on other GABAergic transcripts. Studies 1-3 focused on measuring the mRNA expression of $\mathrm{GABA}_{\mathrm{A}} \mathrm{Rs}$ subunits. Is it possible that other non-GABAergic changes occurred after 3 or 30 days of zolpidem or diazepam? Future experiments should examine if changes in nonGABAergic (eg. NMDAR or AMPAR subunit mRNA) occur after these time points in zolpidem or diazepam groups. Another unexamined question is the temporal duration of 
identified mRNA changes in this study. Experiments looking at mRNA changes greater or less than 1 day after drug cessation might identify acute or more long term changes.

Examination of selective GABA subunits at the protein level indicated mice given zolpidem had decreased expression of total $\alpha 2$ protein in the cortex and mice given diazepam had decreased expression of total $\gamma 2$ protein and decreased intracellular $\alpha 1$ protein in the cortex. Mice given prior zolpidem or diazepam did not show any changes in the cortex of total $\alpha 1, \alpha 3, \mathrm{NR} 1, \mathrm{NR} 2 \mathrm{~A}$, or surface $\alpha 1$ protein expression. There were also no changes in the hippocampus of mice given prior zolpidem or diazepam of total $\alpha 1$ or $\alpha 2$ protein expression. Most in vivo studies of repeated BZ treatment show a decrease in $\alpha 1$ and increases in $\alpha 5, \beta 2$, and $\gamma 2$ protein $[107,109]$. We found a decrease in $\gamma 2$ protein but no changes in $\alpha 1$ protein expression. In vitro studies of long-term diazepam treatment on rat cerebellar neurons found diazepam treatment decreased $\alpha 1$ and $\gamma 2$ protein expression during treatment and after withdrawal increased $\alpha 4$ protein expression $[189,254]$.

Some studies observed decreased number of BZ binding sites after long-term BZ treatment [244]. Receptor uncoupling, internalization, and degradation of $\mathrm{GABA}_{\mathrm{A}} \mathrm{R}$ subunits have been proposed as mechanisms underlying BZ tolerance [151, 197, 282, 283]. While these mechanisms are not fully understood, posttranslational processes, such as phosphorylation, are likely to play key roles $[284,285]$. Other studies have demonstrated that altered mRNA levels induced by chronic BZ exposure can correlate to their respective protein levels in vivo [107, 109]. Thus, it is conceivable that mRNA changes seen in this study coincide with changes in the expression of their respective proteins. However, the relationship between changes in mRNA levels and corresponding protein level is not well characterized. While mRNA serves as an intermediate between DNA and protein, they are subject to posttranscriptional processing by RNA-binding proteins that bind to multiple sites on numerous RNAs to function in diverse timedependent processes [286].

It has been hypothesized that due to zolpidem binding preferentially to $\alpha 1$ $\mathrm{GABA}_{\mathrm{A}} \mathrm{Rs}$ that tolerance would be associated with decreases in $\alpha 1$ and $\gamma 2$ protein expression [105]. We also expected to see a decrease in surface expression of $\alpha 1$ subunit for the same reason. It is possible that the high variability and the failure to see statistical significance with our mRNA and protein experiments were due to small sample sizes. Some of the experiments only had 3-6 mice per group, leading to high variability which may have masked significant results. Future experiments with increased sample size may reduce variability and identify significant differences.

Our study only looked at protein expression after 7 days of drug treatment, however it is possible that changes in protein occur earlier or later. Experiments should be conducted at 3 or 30 days of drug treatment. The study focused on the changes of select $\mathrm{GABA}_{\mathrm{A}} \mathrm{R}$ and NMDAR subunits and it may be possible that changes occur at other signaling transcripts or proteins like KCC2, mGlu5, or PKC [204, 211]. Since there was a decrease in $\alpha 2$ subunit expression after zolpidem treatment, the use of the $\alpha 2$ point mutation mice might show if $\alpha 2-\mathrm{GABA}_{\mathrm{A}} \mathrm{Rs}$ affect zolpidem sedative tolerance. A single 
high dose of diazepam $(30 \mathrm{mg} / \mathrm{kg})$ given to wild-type mice can also produce brainregion-dependent reduction in the expression level of mRNA transcripts involved in the regulation of synaptic plasticity such as c-Fos, CaMKII, and BDNF [287]. Interestingly, many of the transcript changes induced by diazepam in wild-type mice are not altered in mice with $\alpha 1-G A B A_{A} R s$ that have been rendered diazepam-insensitive by point mutations, indicating that these transcript changes are specifically mediated by $\alpha 1-$ containing $\mathrm{GABA}_{\mathrm{A}} \mathrm{Rs}$.

\section{NMDA}

Repeated BZ treatment also affects ionotropic glutamate receptor subunit gene expression. As reviewed elsewhere, alterations in other neurotransmitter systems have been implicated as neuroadaptive mechanisms underlying the development of tolerance and withdrawal to BZ-like drugs, including an upregulation of the glutamatergic system $[151,288]$. In Study 2 it was found that only diazepam treated mice showed a decrease of the mRNA expression of GluR2A subunit mRNA in the hippocampus. There were no other group differences in the hippocampus of GluR1, GluR2B, GluN1 or GluN2 mRNA levels. There were also no group differences for any of the NMDA or AMPA subunit mRNA levels in the cortex, amygdala, or PFC. Our results differ from other studies that find increases in GluR1 and GluR2B, but no increase in GluR2A mRNA levels in the hippocampus after diazepam treatment $[118,119]$. Increases in the expression of AMPA receptor subunit mRNAs and AMPA receptor ligand binding have been reported in a number of brain areas after BZ withdrawal [53,289]. We expected that Study 2 would identify increases in GluR1 and GluR2B mRNA, as found in other studies looking at repeated diazepam in mice $[118,119]$. One difference between our study and others is the time point of sacrifice after drug termination. Our study waited 16-20 hours after last injection whereas others have waited as long as 96 hours after drug termination. Another difference is the method of mRNA analysis. The duration of treatment between Study 2 and past examinations differed. In our study treatment lasted 7 days as opposed to 3 or 14 days $[117,119]$. We used qRT-PCR as opposed to in situ hybridization.

Because other studies have noted changes in NMDAR and AMPAR subunits at varying time points, it is possible that changes in these subunit mRNA may have occurred at 3 or 30 days of treatment. Also is it possible that these changes would only occur during withdrawal. This study focused on ionotropic glutamate receptors but is it possible that our repeated zolpidem and diazepam could result in changes in metabotropic glutamate receptors. One study has found that repeated zolpidem treatment can increase mGluR5 protein [204]. There is evidence that mGlur5 interacts with $\alpha 1-G_{A B A}{ }_{A}$ Rs [205].

At the protein level, zolpidem- and diazepam-treated mice did not show any changes in the cortical GluR1 and GluR2 subunits. This is in contrast to other studies that found increases in GluR1 but not GluR2A protein expression [117]. Electrophysiological data indicate that a single in vivo dose of zolpidem or diazepam can produce an increase in the AMPA/NMDA current ratio and activity of mesolimbic dopamine neurons [167]. Gene-targeted mice lacking the GluR1 subunit of AMPARs show less tolerance to a 
number of the acute behavioral effects of flurazepam (e.g., loss of righting reflex, walking beam impairments) after high-dose treatments [121, 290]. In contrast, greater flumazenil-induced withdrawal-like symptoms are seen after treatment termination. Together, these findings suggesting alterations in AMPA receptors are involved in mediating the processes involved in BZ tolerance and withdrawal.

A variable to consider is the small sample sizes of Studies 1 and 3 which may have resulted in high variation in our data sets. The small sample sizes of our data sets may have masked a possible significant result. Therefore future experiments should have a greater number of mice per group to address this problem. This project focused on mRNA alterations of $\mathrm{GABA}_{\mathrm{A}} \mathrm{R}$ subunits and associated proteins in Studies 1-3 and Study 2 also focused on alterations of NMDA and AMPA receptor subunits. As mentioned above, the focus of this study may have missed other mRNA and protein alterations like those of mGluR, KCC2, PKA, and PKC, that have been found to be associated with BZ and zolpidem tolerance and are known to interact with GABA $\mathrm{Ass}_{\mathrm{A}}$ [204, 205, 209, 211$]$.

\section{Connection Between Sedation, Withdrawal, and Observed Alterations in the mRNA and Protein Levels in the Cortex, PFC, Hippocampus, and Amygdala}

Our results, similar to past studies, found tolerance and cross-tolerance to zolpidem after 7 days of repeated zolpidem and diazepam treatments. We also found that an abrupt end of treatment caused withdrawal anxiety and a reduction in motor activity, a measure of sedation. The most significant changes in mRNA and protein expression occurred during this treatment duration. The cortex and the hippocampus were the only regions that had decreased expression of $\mathrm{GABA}_{\mathrm{A}} \mathrm{R}$ subunits and NMDAR subunits. Both zolpidem and diazepam groups had decreased mRNA levels of $\alpha 1-, \alpha 3-, \beta 2-$, and $\delta$ $\mathrm{GABA}_{\mathrm{A}} \mathrm{R}$ subunits in the cortex. The decreases in $\alpha 1, \alpha 3$, and $\beta 2$ are in line with other studies, but the decrease in cortical $\delta$ mRNA levels has not been previously documented in the cortex or other brain regions. Past studies hypothesize decreases of $\alpha 1, \alpha 3$, and $\beta 2$ may be indicative of a decrease in $\mathrm{GABA}_{\mathrm{A}} \mathrm{Rs}$ in the cortex. A decrease in these subunits may result in a decrease in efficacy of diazepam and zolpidem in the cortex and contribute to our measure of behavioral tolerance observed in the study. The decrease of the $\delta$-subunit mRNA has not been seen in other studies. This finding is surprising given that neither zolpidem nor diazepam bind to $\mathrm{GABA}_{\mathrm{A}} \mathrm{Rs}$ that contain the $\delta$ subunit. Because the location of $\delta$-subunits in the cortex is not firmly established, it is unclear how this affects the development of tolerance. However $\delta$-subunits are known to be incorporated in extrasynaptic $\mathrm{GABA}_{\mathrm{A}} \mathrm{Rs}$ that mediate tonic inhibition, thus reduction may cause an overall decrease in baseline tonic inhibition resulting in an increase in excitatory tone.

The diazepam group had decreased mRNA levels of $\alpha 4-, \beta 1-, \gamma 2$-subunits, GAT, and gephyrin in the cortex. In addition this group showed significant decreases of $\alpha 5$ - and $\beta 3-\mathrm{GABA}_{\mathrm{A}} \mathrm{R}$ subunits and GluN2A subunit in the hippocampus. A decrease in the mRNA of the $\gamma 2$-subunit and gephyrin in the cortex due to repeated diazepam may indicate decrease in BZ-sensitive $\mathrm{GABA}_{\mathrm{A}} \mathrm{Rs}$, as the $\gamma 2$-subunit is necessary for $\mathrm{BZ}$ binding and gephyrin plays a role in maintaining $\mathrm{GABA}_{\mathrm{A}} \mathrm{Rs}$ on the cell surface. It is 
unclear how a decrease in GAT-1 influences tolerance, because a decrease in GAT-1 should lead to a greater concentration of GABA in the synaptic cleft and increased inhibition. It is also unclear why there was a decrease in $\alpha 4$ or $\beta 1 \mathrm{mRNA}$ levels because diazepam nor zolpidem bind to GABA $\mathrm{A}_{\mathrm{A}}$ with these subunits, although a decrease in $\alpha 4$ subunit could result in a decrease in $\mathrm{GABA}_{\mathrm{A}} \mathrm{Rs}$ responsible for tonic inhibition. Other studies tend to see an increase in $\alpha 4$ mRNA levels, which could result in an increase in BZ- and zolpidem-insensitive $\mathrm{GABA}_{\mathrm{A}} \mathrm{Rs}$.

At the protein level our findings did not completely correspond with changes in mRNA levels. Future studies with more refined and sophisticated experimental designs may help resolve discrepancies between mRNA and protein levels seen in our studies. Although some of our results are unresolved, they generally confirm that modulation of neocortical circuits by changes in $\mathrm{GABA}_{\mathrm{A}}$ Rs play a role in the motor impairing effects of PAMs and eventual development of tolerance to zolpidem and diazepam [103].

The diazepam groups had a decrease of $\alpha 5$ - and $\beta 3-\mathrm{GABA}_{\mathrm{A}} \mathrm{R}$ subunits, and the GluN2A subunit in the hippocampus. The decrease of $\alpha 5$ is in line with other studies that examine the effects of repeated diazepam in the hippocampus as diazepam binds to $\mathrm{GABA}_{\mathrm{A}} \mathrm{Rs}$ with this subunit. It is unlikely that a decrease in either of these $\mathrm{GABA}_{\mathrm{A}} \mathrm{R}$ subunits influence sedative cross-tolerance to zolpidem, because $\alpha 5-G_{A B A} R_{s}$ do not participate in the sedative effect of zolpidem. Alterations in $\alpha 5-G_{A B} A_{A} R s$ could, however, alter memory functions, because hippocampal $\alpha 5-\mathrm{GABA}_{\mathrm{A}} \mathrm{Rs}$ are known to be involved in memory and learning. The decrease in GluN2A mRNA levels are novel, though it is unclear how it affects sedative tolerance. Past studies have reported that changes in GluN1 and GluN2B but not GluN2A mRNA and protein levels are associated with the contextual dependent features of PAM tolerance [118, 119, 249]. Because GluN2A is involved in learning in memory, these changes may be associated with contextual dependent features.

Three days of repeated zolpidem did not produce sedative tolerance but did result in a decrease in $\alpha 5$ subunit mRNA levels in the hippocampus. These results only partially supported our hypothesis. As stated above, it is known that changes in hippocampal $\alpha 5-$ $\mathrm{GABA}_{\mathrm{A}}$ Rs do not influence the sedative effects of zolpidem. It is possible that the decrease in $\alpha 5$ subunits is a compensatory change or associated with neuroadaptive changes unrelated to sedation. Although this project found cross-tolerance to zolpidem occurs after 3 days, there were no corresponding changes in mRNA levels. Past studies have found that there are alterations in GluN1 and GluN2B after 4 days $[118,119]$. Thus mechanisms associated sedative cross-tolerance after short-term durations may not be associated with measurable GABAergic alterations.

Thirty days of repeated zolpidem did not produce sedative tolerance but did result in molecular alterations. These results only partially supported our hypothesis. Although 30 days of zolpidem did not result in sedative tolerance, our findings do not rule out the development of withdrawal symptoms afterwards. Thirty days of repeated zolpidem did result in the decrease of $\alpha 1-, \alpha 2-, \alpha 3-, \beta 1-, \beta 2-, \beta 3-, \gamma 1-$, and $\gamma 2$-subunit mRNA levels in the PFC. PFC glutamatergic projections to the amygdala strongly inhibit the amygdala; 
thus, the loss of prefrontal inhibition may increase the glutamatergic drive to the amygdala which can increase anxiety associated with withdrawal symptoms [100]. Although this project found cross-tolerance to zolpidem occurs after 30 days, there were no corresponding changes in GABAergic mRNA levels. Vinkers et al. (2012), have shown that 28 days of diazepam treatment resulted in sedative tolerance to diazepam but no detectable cortical GABAergic mRNA changes and few hippocampal changes [112]. Thus, the presence of sedative tolerance after long term PAM treatments are not always dependent on observable GABAergic alterations.

\section{Limitations}

Our studies provide insight into possible mechanisms behind zolpidem tolerance, although there are limitations to our interpretations. Protein expression was not looked at in all studies. Though $\mathrm{GABA}_{\mathrm{A}} \mathrm{R}$ mRNA and protein expression have been shown to correlate with each other that may not be the case here [107, 109]. We found in Study 1 and 3 that changes in mRNA expression were not associated with the development of cross-tolerance. The diazepam groups in both studies showed cross-tolerance but no changes in mRNA expression, and the zolpidem group in both studies showed no tolerance and minimal changes in mRNA expression. Studies 1 and 3 measured tolerance and mRNA expression in the same mouse whereas Study 2 did not. This would have allowed for correlations on distance and mRNA expression. If prominent changes behavioral and mRNA changes were identified in the same animal in Study 2 correlations may have identified a more definitive relationship between behavioral and neuroadaptive changes. In addition to GABAergic alterations, changes in other neurotransmitter systems and proteins could be involved in zolpidem and diazepam tolerance, but were not investigated in our studies which focused on $\mathrm{GABA}_{\mathrm{A}} \mathrm{R}$ subunits and to a lesser extent AMPAR and NMDAR subunits.

\section{Conclusions}

In summary, this project found that 3 and 30-day zolpidem use did not result in tolerance to zolpidem's sedative effects whereas diazepam treatment at all treatment durations produced cross-tolerance. Three and 30 day treatments of zolpidem but not diazepam resulted in minimal GABAergic mRNA changes. Though there were minor changes in mRNA expression in the PFC with 30-day zolpidem and minor changes in the hippocampus after 3 day zolpidem, these changes are likely independent from sedative tolerance. Seven-day zolpidem and diazepam administration can produce acute deficits in the locomotor-impairing effects of zolpidem and anxiety-like behaviors upon drug termination (spontaneous withdrawal) but not precipitated withdrawal. Our results demonstrated that zolpidem and diazepam treatments have differing effects on $\mathrm{GABA}_{\mathrm{A}} \mathrm{R}$ subunit mRNA expression, mainly in the cortex, which may partially contribute to mechanisms underlying the behavioral phenomena. In general, repeated zolpidem produced fewer changes in the efficacy in of zolpidem in comparison to repeated 
diazepam. However, continued research into tolerance and withdrawal to zolpidem are necessary due to the growing abuse of zolpidem.

\section{Clinical Significance and Implications of Research}

This project showed that 7 days of repeated zolpidem and diazepam resulted in the emergence of sedative tolerance and withdrawal. Along with sedative tolerance, there were significant changes, primarily in the cortex, of GABAergic mRNA and protein levels associated with BZ and zolpidem functioning. Our research indicates the cortical alterations may play a major role in the development of tolerance to zolpidem, similar to what other studies have found. Cortical $\mathrm{GABA}_{\mathrm{A}} \mathrm{Rs}$ appear to be mediate tolerance and the development of future sedative-hypnotics should focus on creating drugs that are either $\mathrm{GABA}_{\mathrm{A}} \mathrm{R}$ subtype selective or drugs that work directly on the arousal system. Subtype selective PAMs that only bind to $\alpha 1-\mathrm{GABA}_{\mathrm{A}}$ Rs with little or no affinity or efficacy to other $\mathrm{GABA}_{A} \mathrm{R}$ subtypes may prevent the development of sedative tolerance and withdrawal. Recently developed orexin receptor antagonists (Suvorexant) that target arousal regions like the locus coeruleus, TMN, and raphe nucleus have been developed and marketed as alternatives to currently used PAMs. Early results suggests orexin antagonists show reduced propensity of typical BZ and zolpidem side effects, like dependence, suggesting they are safer than hypnotic PAMs. Comparing zolpidem to orexin antagonists or other $\alpha 1-\mathrm{GABA}_{\mathrm{A}} \mathrm{R}$ selective PAMs may help us better understand the relationship between molecular alterations and the development of tolerance and withdrawal in humans and better aid in the development of treatment options for the treatment of insomnia. 


\section{LIST OF REFERENCES}

1. Association, A.P., Diagnostic and statistical manual of mental disorders (5th ed.). Arlington, VA: American Psychiatric Publishing, 2013.

2. $\quad$ Roth, T., New developments for treating sleep disorders. J Clin Psychiatry, 2001. 62 Suppl 10: p. 3-4.

3. Ohayon, M.M., Epidemiology of insomnia: what we know and what we still need to learn. Sleep Med Rev, 2002. 6(2): p. 97-111.

4. Lader, M., Benzodiazepines revisited-will we ever learn? Addiction, 2011. 106(12): p. 2086-2109.

5. Beracochea, D., Anterograde and retrograde effects of benzodiazepines on memory. ScientificWorldJournal, 2006. 6: p. 1460-5.

6. Griffiths, R.R. and E.M. Weerts, Benzodiazepine self-administration in humans and laboratory animals--implications for problems of long-term use and abuse. Psychopharmacology (Berl), 1997. 134(1): p. 1-37.

7. Quality, S.A.a.M.H.S.A.C.f.B.H.S.a., The DAWN Report: Highlights of the 2010 Drug Abuse Warning Network (DAWN) Findings on Drug-Related Emergency Department Visits. Rockville, MD, 2012.

8. Bramer, G.R., International statistical classification of diseases and related health problems. Tenth revision. World Health Stat Q, 1988. 41(1): p. 32-6.

9. Neubauer, D.N., The evolution and development of insomnia pharmacotherapies. J Clin Sleep Med, 2007. 3(5 Suppl): p. S11-5.

10. Lopez-Munoz, F., R. Ucha-Udabe, and C. Alamo, The history of barbiturates a century after their clinical introduction. Neuropsychiatr Dis Treat, 2005. 1(4): p. $329-43$.

11. Zakusov, V.V., et al., Further evidence for GABA-ergic mechanisms in the action of benzodiazepines. Arch Int Pharmacodyn Ther, 1977. 229(2): p. 313-26.

12. Klepner, C.A., et al., Resolution of two biochemically and pharmacologically distinct benzodiazepine receptors. Pharmacol Biochem Behav, 1979. 11(4): $\mathrm{p}$. $457-62$.

13. Woods, J.H., J.L. Katz, and G. Winger, Benzodiazepines: use, abuse, and consequences. Pharmacol Rev, 1992. 44(2): p. 151-347.

14. Savic, M.M., et al., Memory effects of benzodiazepines: memory stages and types versus binding-site subtypes. Neural Plast, 2005. 12(4): p. 289-98.

15. Barker, M.J., et al., Persistence of cognitive effects after withdrawal from longterm benzodiazepine use: a meta-analysis. Arch Clin Neuropsychol, 2004. 19(3): p. 437-54.

16. Wright, B.T., C.F. Gluszek, and S.A. Heldt, The effects of repeated zolpidem treatment on tolerance, withdrawal-like symptoms, and GABAA receptor $m R N A s$ profile expression in mice: comparison with diazepam. Psychopharmacology (Berl), 2014. 231(15): p. 2967-79.

17. Sanna, E., et al., Comparison of the effects of zaleplon, zolpidem, and triazolam at various GABA(A) receptor subtypes. Eur J Pharmacol, 2002. 451(2): p. 103-10. 
18. Kralic, J.E., et al., GABA(A) receptor alpha-1 subunit deletion alters receptor subtype assembly, pharmacological and behavioral responses to benzodiazepines and zolpidem. Neuropharmacology, 2002. 43(4): p. 685-94.

19. Mohler, H., J.M. Fritschy, and U. Rudolph, A new benzodiazepine pharmacology. J Pharmacol Exp Ther, 2002. 300(1): p. 2-8.

20. Benca, R.M., Diagnosis and treatment of chronic insomnia: a review. Psychiatr Serv, 2005. 56(3): p. 332-43.

21. Hoffman, J.M., et al., Projecting future drug expenditures in U.S. nonfederal hospitals and clinics--2013. Am J Health Syst Pharm, 2013. 70(6): p. 525-39.

22. World Health Organization Expert Committee on Drug, D., WHO Expert Committee on Drug Dependence. World Health Organ Tech Rep Ser, 2003. 915: p. i-v, 1-26, back cover.

23. Kupfer, D.J. and C.F. Reynolds, 3rd, Management of insomnia. N Engl J Med, 1997. 336(5): p. 341-6.

24. Perrault, G., et al., Lack of tolerance and physical dependence upon repeated treatment with the novel hypnotic zolpidem. J Pharmacol Exp Ther, 1992. 263(1): p. 298-303.

25. Vlainic, J. and D. Pericic, Effects of acute and repeated zolpidem treatment on pentylenetetrazole-induced seizure threshold and on locomotor activity: comparison with diazepam. Neuropharmacology, 2009. 56(8): p. 1124-30.

26. Hajak, G., et al., Abuse and dependence potential for the non-benzodiazepine hypnotics zolpidem and zopiclone: a review of case reports and epidemiological data. Addiction, 2003. 98(10): p. 1371-8.

27. Roehrs, T.A., et al., Twelve months of nightly zolpidem does not lead to dose escalation: a prospective placebo-controlled study. Sleep, 2011. 34(2): p. 207-12.

28. Ebert, B., et al., Gaboxadol -- a different hypnotic profile with no tolerance to sleep EEG and sedative effects after repeated daily dosing. Pharmacol Biochem Behav, 2008. 90(1): p. 113-22.

29. Voss, J., et al., Rotarod studies in the rat of the GABAA receptor agonist gaboxadol: lack of ethanol potentiation and benzodiazepine cross-tolerance. Eur J Pharmacol, 2003. 482(1-3): p. 215-22.

30. Haji Seyed Javadi, S.A., F. Hajiali, and M. Nassiri-Asl, Zolpidem dependency and withdrawal seizure: a case report study. Iran Red Crescent Med J, 2014. 16(11): p. e19926.

31. Drover, D.R., Comparative pharmacokinetics and pharmacodynamics of shortacting hypnosedatives: zaleplon, zolpidem and zopiclone. Clin Pharmacokinet, 2004. 43(4): p. 227-38.

32. Richards, J.G. and J.R. Martin, Binding profiles and physical dependence liabilities of selected benzodiazepine receptor ligands. Brain Res Bull, 1998. 45(4): p. 381-7.

33. Garrigou-Gadenne, D., et al., Pharmacokinetics, brain distribution and pharmaco-electrocorticographic profile of zolpidem, a new hypnotic, in the rat. $\mathrm{J}$ Pharmacol Exp Ther, 1989. 248(3): p. 1283-8.

34. Benavides, J., et al., In vivo interaction of zolpidem with central benzodiazepine (BZD) binding sites (as labeled by [3H]Ro 15-1788) in the mouse brain. 
Preferential affinity of zolpidem for the omega 1 (BZD1) subtype. J Pharmacol

Exp Ther, 1988. 245(3): p. 1033-41.

35. Trenque, T., et al., Pharmacokinetics and brain distribution of zolpidem in the rat after acute and chronic administration. J Pharm Pharmacol, 1994. 46(7): p. 6113.

36. Jinping, Q., et al., Effects of the aqueous extract from Salvia miltiorrhiza Bge on the pharmacokinetics of diazepam and on liver microsomal cytochrome P450 enzyme activity in rats. J Pharm Pharmacol, 2003. 55(8): p. 1163-7.

37. Friedman, H., et al., The pharmacokinetics of diazepam and desmethyldiazepam in rat brain and plasma. Psychopharmacology (Berl), 1986. 88(3): p. 267-70.

38. Elliot, E.E. and J.M. White, Precipitated and spontaneous withdrawal following administration of lorazepam but not zolpidem. Pharmacol Biochem Behav, 2000. 66(2): p. 361-9.

39. Breimer, D.D. and R. Jochemsen, Clinical pharmacokinetics of hypnotic benzodiazepines: a summary. Br J Clin Pharmacol, 1983. 16 Suppl 2: p. 277S278S.

40. Perlis, M.L., et al., Long-term, non-nightly administration of zolpidem in the treatment of patients with primary insomnia. J Clin Psychiatry, 2004. 65(8): p. 1128-37.

41. Walsh, J.K., Erman, M., Erwin, C.W., Jamieson, A., Mahowald, M., Regestein, Q., Scharf, M., Tiegel, P., Vogel, G., and Ware, J.C., Subjective Hypnotic Efficacy of Trazodone and Zolpidem in DSMIII-R Primary Insomnia. Human Psychopharmacology, 1998. 13: p. 191-198.

42. Bonnet, M.H., M. Kramer, and T. Roth, A dose response study of the hypnotic effectiveness of alprazolam and diazepam in normal subjects. Psychopharmacology (Berl), 1981. 75(3): p. 258-61.

43. van Steveninck, A.L., et al., Pharmacokinetic and pharmacodynamic interactions of bretazenil and diazepam with alcohol. Br J Clin Pharmacol, 1996. 41(6): p. 565-73.

44. Troy, S.M., et al., Pharmacokinetic and pharmacodynamic evaluation of the potential drug interaction between venlafaxine and diazepam. J Clin Pharmacol, 1995. 35(4): p. 410-9.

45. Galitz, L.A., S. Jayawardena, and S.A. Furey, Pharmacokinetic effects of simultaneous administration of single-dose gabapentin $500 \mathrm{mg}$ and zolpidem tartrate $10 \mathrm{mg}$ in healthy volunteers: a randomized, open-label, crossover trial. Drugs R D, 2015. 15(1): p. 71-7.

46. National Highway Traffic Safety Administration, D.o.T., Drugs and Human Performance Fact Sheets (DOT HS 809 725). 2014.

47. Von Moltke, L.L., et al., Zolpidem metabolism in vitro: responsible cytochromes, chemical inhibitors, and in vivo correlations. Br J Clin Pharmacol, 1999. 48(1): p. 89-97.

48. Kaplan, S.A., et al., Pharmacokinetic profile of diazepam in man following single intravenous and oral and chronic oral administrations. J Pharm Sci, 1973. 62(11): p. 1789-96. 
49. Greenblatt, D.J., et al., Gender differences in pharmacokinetics and pharmacodynamics of zolpidem following sublingual administration. J Clin Pharmacol, 2014. 54(3): p. 282-90.

50. Greenblatt, D.J., et al., Comparative kinetics and response to the benzodiazepine agonists triazolam and zolpidem: evaluation of sex-dependent differences. $\mathrm{J}$ Pharmacol Exp Ther, 2000. 293(2): p. 435-43.

51. Olubodun, J.O., et al., Zolpidem pharmacokinetic properties in young females: influence of smoking and oral contraceptive use. J Clin Pharmacol, 2002. 42(10): p. 1142-6.

52. Olubodun, J.O., et al., Pharmacokinetic properties of zolpidem in elderly and young adults: possible modulation by testosterone in men. Br J Clin Pharmacol, 2003. 56(3): p. 297-304.

53. Allison, C. and J.A. Pratt, Differential effects of two chronic diazepam treatment regimes on withdrawal anxiety and AMPA receptor characteristics.

Neuropsychopharmacology, 2006. 31(3): p. 602-19.

54. Benavides, J., et al., Comparative autoradiographic distribution of central omega (benzodiazepine) modulatory site subtypes with high, intermediate and low affinity for zolpidem and alpidem. Brain Res, 1993. 604(1-2): p. 240-50.

55. Visser, S.A., et al., Dose-dependent EEG effects of zolpidem provide evidence for $G A B A(A)$ receptor subtype selectivity in vivo. J Pharmacol Exp Ther, 2003.

304(3): p. 1251-7.

56. Wafford, K.A., P.J. Whiting, and J.A. Kemp, Differences in affinity and efficacy of benzodiazepine receptor ligands at recombinant gamma-aminobutyric acidA receptor subtypes. Mol Pharmacol, 1993. 43(2): p. 240-4.

57. Pritchett, D.B., H. Luddens, and P.H. Seeburg, Type I and type II GABAAbenzodiazepine receptors produced in transfected cells. Science, 1989. 245(4924): p. 1389-92.

58. Niddam, R., et al., Autoradiographic localization of [3H]zolpidem binding sites in the rat CNS: comparison with the distribution of [3H]flunitrazepam binding sites. J Neurochem, 1987. 49(3): p. 890-9.

59. Duncan, G.E., et al., Distribution of [3H]zolpidem binding sites in relation to messenger RNA encoding the alpha 1, beta 2 and gamma 2 subunits of GABAA receptors in rat brain. Neuroscience, 1995. 64(4): p. 1113-28.

60. Puia, G., et al., Influence of recombinant gamma-aminobutyric acid-A receptor subunit composition on the action of allosteric modulators of gammaaminobutyric acid-gated Cl-currents. Mol Pharmacol, 1991. 39(6): p. 691-6.

61. Perrais, D. and N. Ropert, Effect of zolpidem on miniature IPSCs and occupancy of postsynaptic GABAA receptors in central synapses. J Neurosci, 1999. 19(2): p. 578-88.

62. Prokic, E.J., et al., Cortical oscillatory dynamics and benzodiazepine-site modulation of tonic inhibition in fast spiking interneurons. Neuropharmacology, 2015. 95: p. 192-205.

63. Kang-Park, M.H., W.A. Wilson, and S.D. Moore, Differential actions of diazepam and zolpidem in basolateral and central amygdala nuclei. Neuropharmacology, 2004. 46(1): p. 1-9. 
64. Krampfl, K., et al., Molecular modulation of recombinant rat alphalbeta2gamma2 GABA(A) receptor channels by diazepam. Neurosci Lett, 1998. 256(3): p. 143-6.

65. $\mathrm{Xu}, \mathrm{J} . Y$. and B.R. Sastry, Benzodiazepine involvement in LTP of the GABA-ergic IPSC in rat hippocampal CA1 neurons. Brain Res, 2005. 1062(1-2): p. 134-43.

66. Depoortere, H., et al., Zolpidem, a novel nonbenzodiazepine hypnotic. I. Neuropharmacological and behavioral effects. J Pharmacol Exp Ther, 1986. 237(2): p. 649-58.

67. Sanger, D.J., D. Joly, and B. Zivkovic, Effects of zolpidem, a new imidazopyridine hypnotic, on the acquisition of conditioned fear in mice. Comparison with triazolam and CL 218,872. Psychopharmacology (Berl), 1986. 90(2): p. 207-10.

68. Sanger, D.J. and B. Zivkovic, Further behavioural evidence for the selective sedative action of zolpidem. Neuropharmacology, 1988. 27(11): p. 1125-30.

69. Sanger, D.J., E. Morel, and G. Perrault, Comparison of the pharmacological profiles of the hypnotic drugs, zaleplon and zolpidem. Eur J Pharmacol, 1996. 313(1-2): p. 35-42.

70. Fahey, J.M., et al., Acute zolpidem administration produces pharmacodynamic and receptor occupancy changes at similar doses. Pharmacol Biochem Behav, 2006. 83(1): p. 21-7.

71. Crestani, F., et al., Mechanism of action of the hypnotic zolpidem in vivo. Br $\mathrm{J}$ Pharmacol, 2000. 131(7): p. 1251-4.

72. Perrault, G., et al., Differences in pharmacological profiles of a new generation of benzodiazepine and non-benzodiazepine hypnotics. Eur J Pharmacol, 1990. 187(3): p. 487-94.

73. Mailliet, F., P. Galloux, and D. Poisson, Comparative effects of melatonin, zolpidem and diazepam on sleep, body temperature, blood pressure and heart rate measured by radiotelemetry in Wistar rats. Psychopharmacology (Berl), 2001. 156(4): p. 417-26.

74. Tobler, I., et al., Diazepam-induced changes in sleep: role of the alpha 1 GABA(A) receptor subtype. Proc Natl Acad Sci U S A, 2001. 98(11): p. 6464-9.

75. Abadie, P., et al., Central benzodiazepine receptor occupancy by zolpidem in the human brain as assessed by positron emission tomography. Eur J Pharmacol, 1996. 295(1): p. 35-44.

76. Duncan, G.E., et al., Species differences in regional patterns of 3H-8-OH-DPAT and $3 \mathrm{H}$-zolpidem binding in the rat and human brain. Pharmacol Biochem Behav, 1998. 60(2): p. 439-48.

77. Hadingham, K.L., et al., Role of the beta subunit in determining the pharmacology of human gamma-aminobutyric acid type A receptors. Mol Pharmacol, 1993. 44(6): p. 1211-8.

78. Smith, A.J., et al., Effect of alpha subunit on allosteric modulation of ion channel function in stably expressed human recombinant gamma-aminobutyric acid(A) receptors determined using (36)Cl ion flux. Mol Pharmacol, 2001. 59(5): p. 110818.

79. Merlotti, L., et al., The dose effects of zolpidem on the sleep of healthy normals. $\mathrm{J}$ Clin Psychopharmacol, 1989. 9(1): p. 9-14. 
80. Drover, D., et al., Pharmacokinetics, pharmacodynamics, and relative pharmacokinetic/pharmacodynamic profiles of zaleplon and zolpidem. Clin Ther, 2000. 22(12): p. 1443-61.

81. Mattila, M.J., et al., Effects of alcohol, zolpidem, and some other sedatives and hypnotics on human performance and memory. Pharmacol Biochem Behav, 1998. 59(4): p. 917-23.

82. Holm, K.J. and K.L. Goa, Zolpidem: an update of its pharmacology, therapeutic efficacy and tolerability in the treatment of insomnia. Drugs, 2000. 59(4): p. 86589.

83. Herzog, C.D., et al., Effects of intraseptal zolpidem and chlordiazepoxide on spatial working memory and high-affinity choline uptake in the hippocampus. Neurobiol Learn Mem, 2000. 73(2): p. 168-79.

84. Zanin, K.A., Lima Patti, C., Tufik, D.P., and Frussa-Filho, F., Zolpidem impairs non-associative memory in mice. Sleep Science, 2011. 4(3): p. 81-87.

85. Huang, M.P., et al., Effects of eszopiclone and zolpidem on sleep-wake behavior, anxiety-like behavior and contextual memory in rats. Behav Brain Res, 2010. 210(1): p. 54-66.

86. Uslaner, J.M., et al., Orexin receptor antagonists differ from standard sleep drugs by promoting sleep at doses that do not disrupt cognition. Sci Transl Med, 2013. 5(179): p. 179ra44.

87. Di Lazzaro, V., et al., GABAA receptor subtype specific enhancement of inhibition in human motor cortex. J Physiol, 2006. 575(Pt 3): p. 721-6.

88. Olsen, R.W. and W. Sieghart, International Union of Pharmacology. LXX. Subtypes of gamma-aminobutyric acid(A) receptors: classification on the basis of subunit composition, pharmacology, and function. Update. Pharmacol Rev, 2008. 60(3): p. 243-60.

89. Blaesse, P., et al., Cation-chloride cotransporters and neuronal function. Neuron, 2009. 61(6): p. 820-38.

90. Rudolph, U. and F. Knoflach, Beyond classical benzodiazepines: novel therapeutic potential of GABAA receptor subtypes. Nat Rev Drug Discov, 2011. 10(9): p. 685-97.

91. Jacob, T.C., S.J. Moss, and R. Jurd, GABA(A) receptor trafficking and its role in the dynamic modulation of neuronal inhibition. Nat Rev Neurosci, 2008. 9(5): p. 331-43.

92. Uusi-Oukari, M. and E.R. Korpi, Regulation of $G A B A(A)$ receptor subunit expression by pharmacological agents. Pharmacol Rev, 2010. 62(1): p. 97-135.

93. Hortnagl, H., et al., Patterns of $m R N A$ and protein expression for 12 GABAA receptor subunits in the mouse brain. Neuroscience, 2013. 236: p. 345-72.

94. Heldt, S.A. and K.J. Ressler, Forebrain and midbrain distribution of major benzodiazepine-sensitive GABAA receptor subunits in the adult C57 mouse as assessed with in situ hybridization. Neuroscience, 2007. 150(2): p. 370-85.

95. Semyanov, A., M.C. Walker, and D.M. Kullmann, GABA uptake regulates cortical excitability via cell type-specific tonic inhibition. Nat Neurosci, 2003. 6(5): p. 484-90.

96. Sieghart, W. and G. Sperk, Subunit composition, distribution and function of GABA(A) receptor subtypes. Curr Top Med Chem, 2002. 2(8): p. 795-816. 
97. Rudolph, U., F. Crestani, and H. Mohler, GABA(A) receptor subtypes: dissecting their pharmacological functions. Trends Pharmacol Sci, 2001. 22(4): p. 188-94.

98. Rudolph, U., et al., Benzodiazepine actions mediated by specific gammaaminobutyric acid(A) receptor subtypes. Nature, 1999. 401(6755): p. 796-800.

99. Low, K., et al., Molecular and neuronal substrate for the selective attenuation of anxiety. Science, 2000. 290(5489): p. 131-4.

100. Isoardi, N.A., et al., Increased fear learning coincides with neuronal dysinhibition and facilitated LTP in the basolateral amygdala following benzodiazepine withdrawal in rats. Neuropsychopharmacology, 2004. 29(10): p. 1852-64.

101. Andrews, N., et al., Evidence that the median raphe nucleus--dorsal hippocampal pathway mediates diazepam withdrawal-induced anxiety. Psychopharmacology (Berl), 1997. 130(3): p. 228-34.

102. Song, J., et al., Benzodiazepine withdrawal-induced glutamatergic plasticity involves up-regulation of GluR1-containing alpha-amino-3-hydroxy-5methylisoxazole-4-propionic acid receptors in Hippocampal CA1 neurons. J Pharmacol Exp Ther, 2007. 322(2): p. 569-81.

103. Zeller, A., et al., Cortical glutamatergic neurons mediate the motor sedative action of diazepam. Mol Pharmacol, 2008. 73(2): p. 282-91.

104. Izzo, E., et al., Glutamic acid decarboxylase and glutamate receptor changes during tolerance and dependence to benzodiazepines. Proc Natl Acad Sci U S A, 2001. 98(6): p. 3483-8.

105. Auta, J., et al., Imidazenil: a low efficacy agonist at alphal-but high efficacy at alpha5-GABAA receptors fail to show anticonvulsant cross tolerance to diazepam or zolpidem. Neuropharmacology, 2008. 55(2): p. 148-53.

106. Heninger, C., et al., Effects of continuous diazepam administration on GABAA subunit mRNA in rat brain. J Mol Neurosci, 1990. 2(2): p. 101-7.

107. Impagnatiello, F., et al., Modifications of gamma-aminobutyric acidA receptor subunit expression in rat neocortex during tolerance to diazepam. Mol Pharmacol, 1996. 49(5): p. 822-31.

108. Longone, P., et al., Reversible modification of GABAA receptor subunit $m R N A$ expression during tolerance to diazepam-induced cognition dysfunction. Neuropharmacology, 1996. 35(9-10): p. 1465-73.

109. Pesold, C., et al., Tolerance to diazepam and changes in GABA(A) receptor subunit expression in rat neocortical areas. Neuroscience, 1997. 79(2): p. 477-87.

110. Tietz, E.I., et al., Temporal and regional regulation of alpha1, beta2 and beta3, but not alpha2, alpha4, alpha5, alpha6, beta1 or gamma2 GABA(A) receptor subunit messenger RNAs following one-week oral flurazepam administration. Neuroscience, 1999. 91(1): p. 327-41.

111. Holt, R.A., A.N. Bateson, and I.L. Martin, Chronic treatment with diazepam or abecarnil differently affects the expression of GABAA receptor subunit $m R N A$ s in the rat cortex. Neuropharmacology, 1996. 35(9-10): p. 1457-63.

112. Vinkers, C.H., et al., GABA(A) receptor alpha subunits differentially contribute to diazepam tolerance after chronic treatment. PLoS One, 2012. 7(8): p. e43054.

113. Zhao, T.J., T.H. Chiu, and H.C. Rosenberg, Reduced expression of gammaaminobutyric acid type A/benzodiazepine receptor gamma 2 and alpha 5 subunit 
$m R N A s$ in brain regions of flurazepam-treated rats. Mol Pharmacol, 1994. 45(4): p. 657-63.

114. Sanna, E., et al., Changes in GABA(A) receptor gene expression associated with selective alterations in receptor function and pharmacology after ethanol withdrawal. J Neurosci, 2003. 23(37): p. 11711-24.

115. Arnot, M.I., et al., GABA(A) receptor gene expression in rat cortex: differential effects of two chronic diazepam treatment regimes. J Neurosci Res, 2001. 64(6): p. 617-25.

116. Wu, Y., et al., Regional changes in [3H]zolpidem binding to brain benzodiazepine receptors in flurazepam tolerant rat: comparison with changes in [3H]flunitrazepam binding. J Pharmacol Exp Ther, 1994. 268(2): p. 675-82.

117. Tsuda, M., et al., Upregulation of NMDA receptor subunit proteins in the cerebral cortex during diazepam withdrawal. Eur J Pharmacol, 1998. 341(2-3): p. $\mathrm{R} 1-2$.

118. Almiron, R.S., M.F. Perez, and O.A. Ramirez, MK-801 prevents the increased $N M D A-N R 1$ and NR2B subunits $m R N A$ expression observed in the hippocampus of rats tolerant to diazepam. Brain Res, 2004. 1008(1): p. 54-60.

119. Perez, M.F., R. Salmiron, and O.A. Ramirez, NMDA-NR1 and-NR2B subunits $m R N A$ expression in the hippocampus of rats tolerant to Diazepam. Behav Brain Res, 2003. 144(1-2): p. 119-24.

120. File, S.E., Rapid development of tolerance to the sedative effects of lorazepam and triazolam in rats. Psychopharmacology (Berl), 1981. 73(3): p. 240-5.

121. Aitta-Aho, T., et al., Reduced benzodiazepine tolerance, but increased flumazenilprecipitated withdrawal in AMPA-receptor GluR-A subunit-deficient mice. Pharmacol Biochem Behav, 2009. 92(2): p. 283-90.

122. Ennaceur, A., et al., Tolerance, sensitization and dependence to diazepam in Balb/c mice exposed to a novel open space anxiety test. Behav Brain Res, 2010. 209(1): p. 154-64.

123. Fernandes, C., et al., The effect of treatment regimen on the development of tolerance to the sedative and anxiolytic effects of diazepam. Psychopharmacology (Berl), 1999. 145(3): p. 251-9.

124. Fernandes, C., S.E. File, and D. Berry, Evidence against oppositional and pharmacokinetic mechanisms of tolerance to diazepam's sedative effects. Brain Res, 1996. 734(1-2): p. 236-42.

125. Joshi, D., Naidu, P., Kulkarni,S., Reversal of Diazepam Tolerance and Withdrawal-Induced Hyperlocomotor Activity and Anxiety by Melatonin in Mice. Annals of Neurosciences, 2006. 13: p. 31-35.

126. Khanna, J.M., A. Chau, and G. Shah, Effect of NMDA antagonists on rapid tolerance to benzodiazepines. Brain Res Bull, 1997. 42(2): p. 99-103.

127. Marin, R.H., N.A. Salvatierra, and O.A. Ramirez, Rapid tolerance to benzodiazepine modifies rat hippocampal synaptic plasticity. Neurosci Lett, 1996. 215(3): p. 149-52.

128. Pugh, S.L., M.S. Boone, and M.W. Emmett-Oglesby, Tolerance, cross-tolerance and withdrawal in rats made dependent on diazepam. J Pharmacol Exp Ther, 1992. 262(2): p. 751-8. 
129. van Rijnsoever, C., et al., Requirement of alpha5-GABAA receptors for the development of tolerance to the sedative action of diazepam in mice. J Neurosci, 2004. 24(30): p. 6785-90.

130. Wong, P.T., Y.L. Yoong, and M.C. Gwee, Acute tolerance to diazepam induced by benzodiazepines. Clin Exp Pharmacol Physiol, 1986. 13(1): p. 1-8.

131. Yoong, Y.L., et al., Acute tolerance to diazepam in mice: pharmacokinetic considerations. Clin Exp Pharmacol Physiol, 1986. 13(2): p. 153-8.

132. File, S.E. and C. Fernandes, Dizocilpine prevents the development of tolerance to the sedative effects of diazepam in rats. Pharmacol Biochem Behav, 1994. 47(4): p. 823-6.

133. Weerts, E.M., et al., Zolpidem physical dependence assessed across increasing doses under a once-daily dosing regimen in baboons. J Pharmacol Exp Ther, 1998. 285(1): p. 41-53.

134. Soyka, M., R. Bottlender, and H.J. Moller, Epidemiological evidence for a low abuse potential of zolpidem. Pharmacopsychiatry, 2000. 33(4): p. 138-41.

135. Rush, C.R., R.W. Baker, and K. Wright, Acute behavioral effects and abuse potential of trazodone, zolpidem and triazolam in humans. Psychopharmacology (Berl), 1999. 144(3): p. 220-33.

136. Victorri-Vigneau, C., et al., Evidence of zolpidem abuse and dependence: results of the French Centre for Evaluation and Information on Pharmacodependence (CEIP) network survey. Br J Clin Pharmacol, 2007. 64(2): p. 198-209.

137. Licata, S.C., et al., Modest abuse-related subjective effects of zolpidem in drugnaive volunteers. Behav Pharmacol, 2011. 22(2): p. 160-6.

138. Griffiths, R.R., et al., Zolpidem behavioral pharmacology in baboons: selfinjection, discrimination, tolerance and withdrawal. J Pharmacol Exp Ther, 1992. 260(3): p. 1199-208.

139. Aragona, M., Abuse, dependence, and epileptic seizures after zolpidem withdrawal: review and case report. Clin Neuropharmacol, 2000. 23(5): p. 281-3.

140. Victorri-Vigneau, C., et al., Pharmacoepidemiological characterisation of zolpidem and zopiclone usage. Eur J Clin Pharmacol, 2013. 69(11): p. 1965-72.

141. Evans, S.M., F.R. Funderburk, and R.R. Griffiths, Zolpidem and triazolam in humans: behavioral and subjective effects and abuse liability. J Pharmacol Exp Ther, 1990. 255(3): p. 1246-55.

142. Sloan, J.W., et al., Diazepam-treated female rats: flumazenil- and PK 11195 induced withdrawal in the hippocampus CA1. Pharmacol Biochem Behav, 1998. 61(1): p. 121-30.

143. Dunworth, S.J., A.N. Mead, and D.N. Stephens, Previous experience of withdrawal from chronic diazepam ameliorates the aversiveness of precipitated withdrawal and reduces withdrawal-induced c-fos expression in nucleus accumbens. Eur J Neurosci, 2000. 12(4): p. 1501-8.

144. Mirza, N.R. and E.O. Nielsen, Do subtype-selective gamma-aminobutyric acid $A$ receptor modulators have a reduced propensity to induce physical dependence in mice? J Pharmacol Exp Ther, 2006. 316(3): p. 1378-85.

145. Keuroghlian, A.S., A.S. Barry, and R.D. Weiss, Circadian dysregulation, zolpidem dependence, and withdrawal seizure in a resident physician performing shift work. Am J Addict, 2012. 21(6): p. 576-7. 
146. Svitek, J., et al., Extensive craving in high dose zolpidem dependency. Prog Neuropsychopharmacol Biol Psychiatry, 2008. 32(2): p. 591-2.

147. Huang, M.C., H.Y. Lin, and C.H. Chen, Dependence on zolpidem. Psychiatry Clin Neurosci, 2007. 61(2): p. 207-8.

148. Sakkas, P., et al., Dependence on zolpidem: a case report. Eur Psychiatry, 1999. 14(6): p. 358-9.

149. Chen, S.C., et al., Detoxification of high-dose zolpidem using cross-titration with an adequate equivalent dose of diazepam. Gen Hosp Psychiatry, 2012. 34(2): p. 210 e5-7.

150. Weerts, E.M. and R.R. Griffiths, Zolpidem self-injection with concurrent physical dependence under conditions of long-term continuous availability in baboons. Behav Pharmacol, 1998. 9(3): p. 285-97.

151. Vinkers, C.H. and B. Olivier, Mechanisms Underlying Tolerance after Long-Term Benzodiazepine Use: A Future for Subtype-Selective GABA(A) Receptor Modulators? Adv Pharmacol Sci, 2012. 2012: p. 416864.

152. VonVoigtlander, P.F. and R.A. Lewis, A rapid screening method for the assessment of benzodiazepine receptor-related physical dependence in mice. Evaluation of benzodiazepine-related agonists and partial agonists. J Pharmacol Methods, 1991. 26(1): p. 1-5.

153. Griffiths, R.R., et al., Self-injection of barbiturates and benzodiazepines in baboons. Psychopharmacology (Berl), 1981. 75(2): p. 101-9.

154. Stewart, R.B., et al., Establishing benzodiazepines as oral reinforcers: midazolam and diazepam self-administration in rhesus monkeys. J Pharmacol Exp Ther, 1994. 271(1): p. 200-11.

155. Griffiths, R.R., et al., Self-injection of barbiturates, benzodiazepines and other sedative-anxiolytics in baboons. Psychopharmacology (Berl), 1991. 103(2): p. 154-61.

156. Ator, N.A., Contributions of GABAA receptor subtype selectivity to abuse liability and dependence potential of pharmacological treatments for anxiety and sleep disorders. CNS Spectr, 2005. 10(1): p. 31-9.

157. Ator, N.A. and R.R. Griffiths, Principles of drug abuse liability assessment in laboratory animals. Drug Alcohol Depend, 2003. 70(3 Suppl): p. S55-72.

158. Bergman, J. and C.E. Johanson, The reinforcing properties of diazepam under several conditions in the rhesus monkey. Psychopharmacology (Berl), 1985. 86(12): p. 108-13.

159. Licata, S.C. and J.K. Rowlett, Abuse and dependence liability of benzodiazepinetype drugs: GABA(A) receptor modulation and beyond. Pharmacol Biochem Behav, 2008. 90(1): p. 74-89.

160. Ator, N.A. and R.R. Griffiths, Oral self-administration of triazolam, diazepam and ethanol in the baboon: drug reinforcement and benzodiazepine physical dependence. Psychopharmacology (Berl), 1992. 108(3): p. 301-12.

161. Meririnne, E., et al., The effects of diazepam and zolpidem on cocaine-and amphetamine-induced place preference. Pharmacol Biochem Behav, 1999. 62(1): p. 159-64. 
162. Ator, N.A., Relation between discriminative and reinforcing effects of midazolam, pentobarbital, chlordiazepoxide, zolpidem, and imidazenil in baboons.

Psychopharmacology (Berl), 2002. 163(3-4): p. 477-87.

163. Carden, S.E. and E.E. Coons, Diazepam's impact on self-stimulation but not stimulation-escape suggests hedonic modulation. Behav Neurosci, 1990. 104(1): p. 56-61.

164. Straub, C.J., W.A. Carlezon, Jr., and U. Rudolph, Diazepam and cocaine potentiate brain stimulation reward in C57BL/6J mice. Behav Brain Res, 2010. 206(1): p. 17-20.

165. Reynolds, L.M., et al., Differential roles of GABA(A) receptor subtypes in benzodiazepine-induced enhancement of brain-stimulation reward.

Neuropsychopharmacology, 2012. 37(11): p. 2531-40.

166. Spyraki, C., A. Kazandjian, and D. Varonos, Diazepam-induced place preference conditioning: appetitive and antiaversive properties. Psychopharmacology (Berl), 1985. 87(2): p. 225-32.

167. Heikkinen, A.E., T.P. Moykkynen, and E.R. Korpi, Long-lasting modulation of glutamatergic transmission in VTA dopamine neurons after a single dose of benzodiazepine agonists. Neuropsychopharmacology, 2009. 34(2): p. 290-8.

168. Tan, K.R., et al., Neural bases for addictive properties of benzodiazepines. Nature, 2010. 463(7282): p. 769-74.

169. Tan, K.R., U. Rudolph, and C. Luscher, Hooked on benzodiazepines: GABAA receptor subtypes and addiction. Trends Neurosci, 2011. 34(4): p. 188-97.

170. Le Moal, M. and G.F. Koob, Drug addiction: pathways to the disease and pathophysiological perspectives. Eur Neuropsychopharmacol, 2007. 17(6-7): p. 377-93.

171. Abuse, N.I.o.D., Commonly Abused Prescription Drugs Chart September 2002.

172. Substance Abuse and Mental Health Services Administration, D.A.W.N., National Estimates of Drug-Related Emergency Department Visits. HHS Publication No. (SMA) 13-4760,

DAWN Series D-39. Rockville, MD: Substance Abuse and Mental Health Services Administration, 2013., 2011.

173. World Health, O., WHO Expert Committee on Drug Dependence. Thirty-second report. World Health Organ Tech Rep Ser, 2001. 903: p. i-v, 1-26.

174. Gericke, C.A. and A.C. Ludolph, Chronic abuse of zolpidem. JAMA, 1994. 272(22): p. 1721-2.

175. Liappas, I.A., et al., Three cases of zolpidem dependence treated with fluoxetine: the serotonin hypothesis. World J Biol Psychiatry, 2003. 4(2): p. 93-6.

176. Savic, M.M., et al., Bidirectional effects of benzodiazepine binding site ligands in the passive avoidance task: differential antagonism by flumazenil and beta-CCt. Behav Brain Res, 2005. 158(2): p. 293-300.

177. Zanin, K.A., et al., Effects of zolpidem on sedation, anxiety, and memory in the plus-maze discriminative avoidance task. Psychopharmacology (Berl), 2013. 226(3): p. 459-74.

178. Krasowski, M.D.a.B., J., Forensic Aspects of Hypnotic Drugs. Behavioral Health, 2015. 1(2): p. 1-8. 
179. Dingemanse, J., et al., Comparative tolerability, pharmacodynamics, and pharmacokinetics of a metabolite of a quinolizinone hypnotic and zolpidem in healthy subjects. Drug Metab Dispos, 2000. 28(12): p. 1411-6.

180. Chopra, A., et al., Para-suicidal amnestic behavior associated with chronic zolpidem use: implications for patient safety. Psychosomatics, 2013. 54(5): p. 498-501.

181. Toner, L.C., et al., Central nervous system side effects associated with zolpidem treatment. Clin Neuropharmacol, 2000. 23(1): p. 54-8.

182. Cubala, W.J.a.G., A., Sleep Related Amnestic Behaviors due to Zolpidem. Bulletin of Clinincal Psychopharmacology, 2014. 24(2): p. 188-94.

183. Sansone, R.A. and L.A. Sansone, Zolpidem, somnambulism, and nocturnal eating. Gen Hosp Psychiatry, 2008. 30(1): p. 90-1.

184. Lai, M.M., et al., Long-term use of zolpidem increases the risk of major injury: a population-based cohort study. Mayo Clin Proc, 2014. 89(5): p. 589-94.

185. Olsen, R.W., et al., Plasticity of GABAA receptors in brains of rats treated with chronic intermittent ethanol. Neurochem Res, 2005. 30(12): p. 1579-88.

186. Follesa, P., et al., Changes in $G A B A(A)$ receptor gene expression induced by withdrawal of, but not by long-term exposure to, zaleplon or zolpidem. Neuropharmacology, 2002. 42(2): p. 191-8.

187. Jembrek, M.J., J. Vlainic, and J. Suran, Zolpidem withdrawal induced uncoupling of $G A B A A$ receptors in vitro associated with altered $G A B A A$ receptor subunit $m R N A$ expression. Acta Neurobiol Exp (Wars), 2015. 75(2): p. 160-71.

188. Biggio, G., et al., Molecular mechanisms of tolerance to and withdrawal of $G A B A(A)$ receptor modulators. Eur Neuropsychopharmacol, 2003. 13(6): p. 41123.

189. Follesa, P., et al., Increase in expression of the $G A B A(A)$ receptor alpha(4) subunit gene induced by withdrawal of, but not by long-term treatment with, benzodiazepine full or partial agonists. Brain Res Mol Brain Res, 2001. 92(1-2): p. 138-48.

190. Primus, R.J., et al., Allosteric uncoupling after chronic benzodiazepine exposure of recombinant gamma-aminobutyric acid(A) receptors expressed in Sf9 cells: ligand efficacy and subtype selectivity. J Pharmacol Exp Ther, 1996. 276(3): p. 882-90.

191. Vlainic, J., et al., Differential effects of short- and long-term zolpidem treatment on recombinant alphalbeta2gamma2s subtype of $G A B A(A)$ receptors in vitro. Acta Pharmacol Sin, 2012. 33(12): p. 1469-76.

192. Friedman, L.K., T.T. Gibbs, and D.H. Farb, gamma-aminobutyric acidA receptor regulation: heterologous uncoupling of modulatory site interactions induced by chronic steroid, barbiturate, benzodiazepine, or GABA treatment in culture. Brain Res, 1996. 707(1): p. 100-9.

193. Vlainic, J., et al., The effects of zolpidem treatment and withdrawal on the in vitro expression of recombinant alphalbeta2gamma2s $G A B A(A)$ receptors expressed in HEK 293 cells. Naunyn Schmiedebergs Arch Pharmacol, 2010. 382(3): p. 20112.

194. Gallager, D.W., et al., Chronic benzodiazepine treatment decreases postsynaptic GABA sensitivity. Nature, 1984. 308(5954): p. 74-7. 
195. Marley, R.J. and D.W. Gallager, Chronic diazepam treatment produces regionally specific changes in GABA-stimulated chloride influx. Eur J Pharmacol, 1989.

159(3): p. 217-23.

196. Liang, J., et al., Chronic intermittent ethanol-induced switch of ethanol actions from extrasynaptic to synaptic hippocampal GABAA receptors. J Neurosci, 2006. 26(6): p. 1749-58.

197. Ali, N.J. and R.W. Olsen, Chronic benzodiazepine treatment of cells expressing recombinant $G A B A(A)$ receptors uncouples allosteric binding: studies on possible mechanisms. J Neurochem, 2001. 79(5): p. 1100-8.

198. Tehrani, M.H. and E.M. Barnes, Jr., Sequestration of gamma-aminobutyric acidA receptors on clathrin-coated vesicles during chronic benzodiazepine administration in vivo. J Pharmacol Exp Ther, 1997. 283(1): p. 384-90.

199. Kumar, S., et al., Chronic ethanol consumption enhances internalization of alphal subunit-containing GABAA receptors in cerebral cortex. J Neurochem, 2003. 86(3): p. 700-8.

200. Tsuda, M., T. Suzuki, and M. Misawa, Recovery of decreased seizure threshold for pentylenetetrazole during diazepam withdrawal by NMDA receptor antagonists. Eur J Pharmacol, 1997. 324(1): p. 63-6.

201. Tsuda, M., et al., Hypersusceptibility to DMCM-induced seizures during diazepam withdrawal in mice: evidence for upregulation of NMDA receptors. Naunyn Schmiedebergs Arch Pharmacol, 1998. 357(3): p. 309-15.

202. Bonavita, C., et al., Adaptive changes in the rat hippocampal glutamatergic neurotransmission are observed during long-term treatment with lorazepam. Psychopharmacology (Berl), 2003. 166(2): p. 163-7.

203. Van Sickle, B.J., et al., Chronic benzodiazepine administration alters hippocampal CA1 neuron excitability: NMDA receptor function and expression(1). Neuropharmacology, 2002. 43(4): p. 595-606.

204. Shibasaki, M., et al., Implication of $m$ Glu 5 receptor in the enhancement of morphine-induced hyperlocomotion under chronic treatment with zolpidem. Eur J Pharmacol, 2014. 738: p. 360-7.

205. Besheer, J. and C.W. Hodge, Pharmacological and anatomical evidence for an interaction between $m G l u R 5$ - and GABA(A) alphal-containing receptors in the discriminative stimulus effects of ethanol. Neuropsychopharmacology, 2005. 30(4): p. 747-57.

206. Licata, S.C., et al., Alterations in brain-derived neurotrophic factor in the mouse hippocampus following acute but not repeated benzodiazepine treatment. PLoS One, 2013. 8(12): p. e84806.

207. Lund, I.V., et al., BDNF selectively regulates GABAA receptor transcription by activation of the JAK/STAT pathway. Sci Signal, 2008. 1(41): p. ra9.

208. Brunig, I., et al., BDNF reduces miniature inhibitory postsynaptic currents by rapid downregulation of $G A B A(A)$ receptor surface expression. Eur J Neurosci, 2001. 13(7): p. 1320-8.

209. Poisbeau, P., et al., Modulation of synaptic GABAA receptor function by PKA and $P K C$ in adult hippocampal neurons. J Neurosci, 1999. 19(2): p. 674-83.

210. $\mathrm{Hu}, \mathrm{Y}$. , et al., Surface expression of GABAA receptors is transcriptionally controlled by the interplay of cAMP-response element-binding protein and its 
binding partner inducible cAMP early repressor. J Biol Chem, 2008. 283(14): p. 9328-40.

211. Shibasaki, M., et al., Involvement of the $\mathrm{K}^{+-C l} \mathrm{Cl}$ - co-transporter $\mathrm{KCC} 2$ in the sensitization to morphine-induced hyperlocomotion under chronic treatment with zolpidem in the mesolimbic system. J Neurochem, 2013. 125(5): p. 747-55.

212. Godwin, S.A., et al., Clinical policy: procedural sedation and analgesia in the emergency department. Ann Emerg Med, 2014. 63(2): p. 247-58 e18.

213. Foster, A.C., et al., In vivo pharmacological characterization of indiplon, a novel pyrazolopyrimidine sedative-hypnotic. J Pharmacol Exp Ther, 2004. 311(2): p. 547-59.

214. McKernan, R.M., et al., Sedative but not anxiolytic properties of benzodiazepines are mediated by the GABA(A) receptor alphal subtype. Nat Neurosci, 2000. 3(6): p. 587-92.

215. Winsky-Sommerer, R., Role of GABAA receptors in the physiology and pharmacology of sleep. Eur J Neurosci, 2009. 29(9): p. 1779-94.

216. Rosenwasser, A.M., Functional neuroanatomy of sleep and circadian rhythms. Brain Res Rev, 2009. 61(2): p. 281-306.

217. Nelson, L.E., et al., The sedative component of anesthesia is mediated by GABA(A) receptors in an endogenous sleep pathway. Nat Neurosci, 2002. 5(10): p. $979-84$.

218. Kopp, C., et al., Diazepam-induced changes on sleep and the EEG spectrum in mice: role of the alpha3-GABA(A) receptor subtype. Eur J Neurosci, 2003. 17(10): p. 2226-30.

219. Kopp, C., U. Rudolph, and I. Tobler, Sleep EEG changes after zolpidem in mice. Neuroreport, 2004. 15(14): p. 2299-302.

220. Takakusaki, K., Forebrain control of locomotor behaviors. Brain Res Rev, 2008. 57(1): p. 192-8.

221. Graybiel, A.M., The basal ganglia. Curr Biol, 2000. 10(14): p. R509-11.

222. Siemiatkowski, M., et al., Effects of buspirone, diazepam, and zolpidem on open field behavior, and brain [3H]muscimol binding after buspirone pretreatment. Pharmacol Biochem Behav, 2000. 66(3): p. 645-51.

223. DeLorey, T.M., et al., Influence of benzodiazepine binding site ligands on fearconditioned contextual memory. Eur J Pharmacol, 2001. 426(1-2): p. 45-54.

224. Griebel, G., et al., New evidence that the pharmacological effects of benzodiazepine receptor ligands can be associated with activities at different $B Z$ (omega) receptor subtypes. Psychopharmacology (Berl), 1999. 146(2): p. 205-13.

225. Savic, M.M., et al., The differential role of alphal-and alpha5-containing GABA(A) receptors in mediating diazepam effects on spontaneous locomotor activity and water-maze learning and memory in rats. Int $\mathrm{J}$ Neuropsychopharmacol, 2009. 12(9): p. 1179-93.

226. Vogel, H.G.a.V., W.H., Drug Discovery and Evaluation: Pharmacological Assays. 2002, New York: Springer.

227. Acikmese, B., et al., Evaluation of anxiolytic effect and withdrawal anxiety in chronic intermittent diazepam treatment in rats. Behav Pharmacol, 2012. 23(2): p. 215-9. 
228. Divljakovic, J., et al., betaCCT, an antagonist selective for alpha(1)GABA(A) receptors, reverses diazepam withdrawal-induced anxiety in rats. Brain Res Bull, 2013. 91: p. 1-7.

229. Kovacevic, J., et al., Duration of treatment and activation of alphal-containing GABAA receptors variably affect the level of anxiety and seizure susceptibility after diazepam withdrawal in rats. Brain Res Bull, 2014. 104: p. 1-6.

230. Kulkarni, S.K., A. Kaushal, and A. Dhir, Effect of withdrawal of diazepam or morphine treatment on gastric motility (charcoal meal test) in mice: possible role of different central and peripheral receptors. Indian J Exp Biol, 2007. 45(7): p. 642-8.

231. Monti, M.C., et al., Impact of contextual cues in the expression of the memory associated with diazepam withdrawal: involvement of hippocampal PKMzeta in vivo, and Arc expression and LTP in vitro. Eur J Neurosci, 2012. 36(8): p. 311825.

232. Davis, M., The role of the amygdala in conditioned and unconditioned fear and anxiety. In: Aggleton JP (ed) The amygdala : A functional analysis. Oxford University Press, New York,, 2000: p. 213-287.

233. Loscher, W. and W.S. Schwark, Development of tolerance to the anticonvulsant effect of diazepam in amygdala-kindled rats. Exp Neurol, 1985. 90(2): p. 373-84.

234. Holt, R.A., A.N. Bateson, and I.L. Martin, Chronic zolpidem treatment alters GABA(A) receptor $m R N A$ levels in the rat cortex. Eur J Pharmacol, 1997. 329(23): p. 129-32.

235. Clark, J.D., et al., Special Report: The 1996 Guide for the Care and Use of Laboratory Animals. ILAR J, 1997. 38(1): p. 41-48.

236. Siegel, S., Pharmacological conditioning and drug effects. In: Goudie AJ, Emmett-Oglesby MW (eds) Psychoactive drugs: Tolerance and sensitization., 1989. Humana Press, Totowa: p. 115-180.

237. Stock, H., et al., Lack of sex differences in anxiety behaviors during precipitated benzodiazepine withdrawal in rats. Physiol Behav, 1999. 66(1): p. 125-30.

238. Ugalde, M., et al., Isobolographic analysis of the sedative interaction between six central nervous system depressant drugs and Valeriana edulis hydroalcoholic extract in mice. J Pharm Pharmacol, 2005. 57(5): p. 631-9.

239. King, D.A., M.E. Bouton, and R.E. Musty, Associative control of tolerance to the sedative effects of a short-acting benzodiazepine. Behav Neurosci, 1987. 101(1): p. 104-14.

240. Pieri, L., et al., Pharmacology of midazolam. Arzneimittelforschung, 1981. 31(12a): p. 2180-201.

241. Giulietti, A., et al., An overview of real-time quantitative PCR: applications to quantify cytokine gene expression. Methods, 2001. 25(4): p. 386-401.

242. Boudreau, A.C., et al., A protein cross-linking assay for measuring cell surface expression of glutamate receptor subunits in the rodent brain after in vivo treatments. Curr Protoc Neurosci, 2012. Chapter 5: p. Unit 530 1-19.

243. Galpern, W.R., et al., Chronic benzodiazepine administration. VII. Behavioral tolerance and withdrawal and receptor alterations associated with clonazepam administration. Psychopharmacology (Berl), 1991. 104(2): p. 225-30. 
244. Miller, L.G., et al., Chronic benzodiazepine administration. IV. Rapid development of tolerance and receptor downregulation associated with alprazolam administration. Biochem Pharmacol, 1989. 38(21): p. 3773-7.

245. Fitzgerald, A.C., B.T. Wright, and S.A. Heldt, The behavioral pharmacology of zolpidem: evidence for the functional significance of alphal-containing GABA(A) receptors. Psychopharmacology (Berl), 2014. 231(9): p. 1865-96.

246. Steppuhn, K.G. and L. Turski, Diazepam dependence prevented by glutamate antagonists. Proc Natl Acad Sci U S A, 1993. 90(14): p. 6889-93.

247. Cohen, C. and D.J. Sanger, Tolerance, cross-tolerance and dependence measured by operant responding in rats treated with triazolam via osmotic pumps. Psychopharmacology (Berl), 1994. 115(1-2): p. 86-94.

248. File, S.E., Tolerance to the behavioral actions of benzodiazepines. Neurosci Biobehav Rev, 1985. 9(1): p. 113-21.

249. Marin, R.H., et al., Preexposure to drug administration context blocks the development of tolerance to sedative effects of diazepam. Pharmacol Biochem Behav, 1999. 64(3): p. 473-7.

250. Petursson, H., The benzodiazepine withdrawal syndrome. Addiction, 1994. 89(11): p. 1455-9.

251. Cubala, W.J. and J. Landowski, Seizure following sudden zolpidem withdrawal. Prog Neuropsychopharmacol Biol Psychiatry, 2007. 31(2): p. 539-40.

252. Kripke, D.F., Greater incidence of depression with hypnotic use than with placebo. BMC Psychiatry, 2007. 7: p. 42.

253. Sethi, P.K. and D.C. Khandelwal, Zolpidem at supratherapeutic doses can cause drug abuse, dependence and withdrawal seizure. J Assoc Physicians India, 2005. 53: p. 139-40.

254. Brown, M.J. and D.R. Bristow, Molecular mechanisms of benzodiazepine-induced down-regulation of GABAA receptor alpha 1 subunit protein in rat cerebellar granule cells. Br J Pharmacol, 1996. 118(5): p. 1103-10.

255. Allison, C., L.A. Claase, and J.A. Pratt, Diazepam withdrawal-induced anxiety and place aversion in the rat: differential effects of two chronic diazepam treatment regimes. Behav Pharmacol, 2002. 13(5-6): p. 417-25.

256. File, S.E., The history of benzodiazepine dependence: a review of animal studies. Neurosci Biobehav Rev, 1990. 14(2): p. 135-46.

257. Lukas, S.E. and R.R. Griffiths, Precipitated diazepam withdrawal in baboons: effects of dose and duration of diazepam exposure. Eur J Pharmacol, 1984. 100(2): p. 163-71.

258. Yanagita, T. and S. Takahashi, Development of tolerance to and physical dependence on barbiturates in rhesus monkeys. J Pharmacol Exp Ther, 1970. 172(1): p. 163-9.

259. Metten, P., J.K. Belknap, and J.C. Crabbe, Drug withdrawal convulsions and susceptibility to convulsants after short-term selective breeding for acute ethanol withdrawal. Behav Brain Res, 1998. 95(1): p. 113-22.

260. Renger, J.J., et al., Sub-chronic administration of zolpidem affects modifications to rat sleep architecture. Brain Res, 2004. 1010(1-2): p. 45-54. 
261. Murphy, H.M., C. Ihekoronze, and C.H. Wideman, Zolpidem-induced changes in activity, metabolism, and anxiety in rats. Pharmacol Biochem Behav, 2011. 98(1): p. 81-6.

262. Ward, B.O. and D.N. Stephens, Sensitisation of withdrawal signs following repeated withdrawal from a benzodiazepine: differences between measures of anxiety and seizure sensitivity. Psychopharmacology (Berl), 1998. 135(4): p. 34252.

263. Lau, C.E., et al., The effect of zolpidem on operant behavior and its relation to pharmacokinetics after intravenous and subcutaneous administration: concentration-effect relations. Behav Pharmacol, 2002. 13(2): p. 93-103.

264. Markowitz, G.J., et al., The pharmacokinetics of commonly used antiepileptic drugs in immature CD1 mice. Neuroreport, 2010. 21(6): p. 452-6.

265. Walker, M.C., et al., Comparison of single- and repeated-dose pharmacokinetics of diazepam. Epilepsia, 1998. 39(3): p. 283-9.

266. Diaz-Garcia, J.M., J. Oliver-Botana, and D. Fos-Galve, Pharmacokinetics of diazepam in the rat: influence of a carbon tetrachloride-induced hepatic injury. $\mathrm{J}$ Pharm Sci, 1992. 81(8): p. 768-72.

267. Auta, J., et al., Imidazenil, a partial positive allosteric modulator of GABAA receptors, exhibits low tolerance and dependence liabilities in the rat. $\mathrm{J}$ Pharmacol Exp Ther, 1994. 270(3): p. 1262-9.

268. Smith, P.F.a.D., C.L., The behavioural effects of long-term use of benzodiazepine sedative and hypnotic drugs: what can be learned from animal studies? New Zealand Journal of Psychology, 1994. 23: p. 48-63.

269. Kliethermes, C.L., et al., Selection for pentobarbital withdrawal severity: correlated differences in withdrawal from other sedative drugs. Brain Res, 2004. 1009(1-2): p. 17-25.

270. Petroski, R.E., et al., Indiplon is a high-affinity positive allosteric modulator with selectivity for alphal subunit-containing GABAA receptors. J Pharmacol Exp Ther, 2006. 317(1): p. 369-77.

271. Fahey, J.M., et al., In situ hybridization histochemistry as a method to assess GABA(A) receptor subunit $m R N A$ expression following chronic alprazolam administration. J Psychopharmacol, 1999. 13(3): p. 211-8.

272. Kang, I. and L.G. Miller, Decreased GABAA receptor subunit mRNA concentrations following chronic lorazepam administration. Br J Pharmacol, 1991. 103(2): p. 1285-7.

273. Pratt, J.A., R.R. Brett, and D.J. Laurie, Benzodiazepine dependence: from neural circuits to gene expression. Pharmacol Biochem Behav, 1998. 59(4): p. 925-34.

274. Asan, E., et al., The corticotropin-releasing factor (CRF)-system and monoaminergic afferents in the central amygdala: investigations in different mouse strains and comparison with the rat. Neuroscience, 2005. 131(4): p. 95367.

275. Blanchard, D.C., G. Griebel, and R.J. Blanchard, Mouse defensive behaviors: pharmacological and behavioral assays for anxiety and panic. Neurosci Biobehav Rev, 2001. 25(3): p. 205-18.

276. Jardim, M.C., et al., Evaluation of the elevated T-maze as an animal model of anxiety in the mouse. Brain Res Bull, 1999. 48(4): p. 407-11. 
277. Korpi, E.R., et al., Altered receptor subtypes in the forebrain of GABA(A) receptor delta subunit-deficient mice: recruitment of gamma 2 subunits. Neuroscience, 2002. 109(4): p. 733-43.

278. Pirker, S., et al., GABA(A) receptors: immunocytochemical distribution of 13 subunits in the adult rat brain. Neuroscience, 2000. 101(4): p. 815-50.

279. Araujo, F., et al., Molecular and pharmacological characterization of native cortical gamma-aminobutyric acidA receptors containing both alphal and alpha3 subunits. J Biol Chem, 1996. 271(44): p. 27902-11.

280. Atack, J.R., et al., Anxiogenic properties of an inverse agonist selective for alpha 3 subunit-containing GABA A receptors. Br J Pharmacol, 2005. 144(3): p. 357-66.

281. Crestani, F., et al., Molecular targets for the myorelaxant action of diazepam. Mol Pharmacol, 2001. 59(3): p. 442-5.

282. Gravielle, M.C., et al., GABA induces activity dependent delayed-onset uncoupling of GABA/benzodiazepine site interactions in neocortical neurons. $\mathrm{J}$ Biol Chem, 2005. 280(22): p. 20954-60.

283. Jacob, T.C., et al., Benzodiazepine treatment induces subtype-specific changes in GABA(A) receptor trafficking and decreases synaptic inhibition. Proc Natl Acad Sci U S A, 2012. 109(45): p. 18595-600.

284. Bateson, A.N., Basic pharmacologic mechanisms involved in benzodiazepine tolerance and withdrawal. Curr Pharm Des, 2002. 8(1): p. 5-21.

285. Kittler, J.T., et al., Constitutive endocytosis of GABAA receptors by an association with the adaptin AP2 complex modulates inhibitory synaptic currents in hippocampal neurons. J Neurosci, 2000. 20(21): p. 7972-7.

286. Glisovic, T., et al., RNA-binding proteins and post-transcriptional gene regulation. FEBS Lett, 2008. 582(14): p. 1977-86.

287. Huopaniemi, L., et al., Diazepam-induced adaptive plasticity revealed by alpha1 GABAA receptor-specific expression profiling. J Neurochem, 2004. 88(5): p. $1059-67$.

288. Allison, C. and J.A. Pratt, Neuroadaptive processes in GABAergic and glutamatergic systems in benzodiazepine dependence. Pharmacol Ther, 2003. 98(2): p. 171-95.

289. Allison, C., et al., alpha-Amino-3-hydroxy-5-methylisoxazole-4-propionate receptor autoradiography in mouse brain after single and repeated withdrawal from diazepam. Eur J Neurosci, 2005. 21(4): p. 1045-56.

290. Zamanillo, D., et al., Importance of AMPA receptors for hippocampal synaptic plasticity but not for spatial learning. Science, 1999. 284(5421): p. 1805-11. 
APPENDIX. MRNA EXPRESSION AFTER 1 DAY OF VEH, DZP, AND ZOLP

\begin{tabular}{|c|c|c|c|c|c|c|}
\hline \multirow{2}{*}{$\underline{\text { Region }}$} & \multicolumn{2}{|c|}{ Vehicle } & \multicolumn{2}{|c|}{ Zolpidem } & \multicolumn{2}{|c|}{ Diazepam } \\
\hline & Mean & SEM & Mean & SEM & Mean & SEM \\
\hline$\alpha 1$ & 1.00 & 0.04 & 1.01 & 0.06 & 0.99 & 0.05 \\
\hline$\alpha 2$ & 1.00 & 0.08 & 1.02 & 0.09 & 1.08 & 0.07 \\
\hline$\alpha 3$ & 1.00 & 0.10 & 1.02 & 0.08 & 1.02 & 0.09 \\
\hline$\alpha 5$ & 1.00 & 0.07 & 1.00 & 0.09 & 1.02 & 0.08 \\
\hline$\beta 2$ & 1.00 & 0.09 & 0.92 & 0.06 & 0.87 & 0.08 \\
\hline$\gamma 2$ & 1.00 & 0.09 & 1.01 & 0.03 & 0.95 & 0.05 \\
\hline GAT-1 & 1.00 & 0.06 & 0.97 & 0.07 & 0.94 & 0.08 \\
\hline Hippocampus & Mean & SEM & Mean & SEM & Mean & SEM \\
\hline$\alpha 1$ & 1.00 & 0.19 & 1.31 & 0.22 & 1.40 & 0.17 \\
\hline$\alpha 2$ & 1.00 & 0.12 & 0.94 & 0.15 & 1.08 & 0.11 \\
\hline$\alpha 3$ & 1.00 & 0.30 & 0.80 & 0.07 & 0.84 & 0.22 \\
\hline$\alpha 5$ & 1.00 & 0.27 & 1.41 & 0.17 & 1.49 & 0.28 \\
\hline$\beta 2$ & 1.00 & 0.16 & 1.22 & 0.14 & 1.15 & 0.18 \\
\hline$\gamma^{2}$ & 1.00 & 0.10 & 1.01 & 0.13 & 1.01 & 0.05 \\
\hline GAT-1 & 1.00 & 0.22 & 0.65 & 0.10 & 0.76 & 0.05 \\
\hline Amygdala & Mean & SEM & Mean & SEM & Mean & SEM \\
\hline$\alpha 1$ & 1.00 & 0.08 & 1.12 & 0.10 & 1.21 & 0.05 \\
\hline$\alpha 2$ & 1.00 & 0.14 & 1.23 & 0.21 & 1.34 & 0.10 \\
\hline$\alpha 3$ & 1.00 & 0.21 & 1.43 & 0.15 & 1.41 & 0.09 \\
\hline$\alpha 5$ & 1.00 & 0.34 & 0.75 & 0.09 & 0.94 & 0.13 \\
\hline$\beta 2$ & 1.00 & 0.11 & 1.01 & 0.12 & 1.07 & 0.06 \\
\hline$\gamma^{2}$ & 1.00 & 0.06 & 1.05 & 0.11 & 1.10 & 0.03 \\
\hline GAT-1 & 1.00 & 0.08 & 0.90 & 0.09 & 1.16 & 0.06 \\
\hline Amygdala & Mean & SEM & Mean & SEM & Mean & SEM \\
\hline$\alpha 1$ & 1.00 & 0.10 & 0.85 & 0.23 & 0.77 & 0.02 \\
\hline$\alpha 2$ & 1.00 & 0.07 & 0.94 & 0.27 & 0.83 & 0.06 \\
\hline$\alpha 3$ & 1.00 & 0.14 & 0.70 & 0.36 & 0.32 & 0.14 \\
\hline$\alpha 5$ & 1.00 & 0.17 & 1.28 & 0.10 & 1.79 & 0.28 \\
\hline$\beta 2$ & 1.00 & 0.08 & 0.84 & 0.29 & 0.62 & 0.06 \\
\hline$\gamma 2$ & 1.00 & 0.07 & 0.90 & 0.19 & 0.78 & 0.02 \\
\hline GAT-1 & 1.00 & 0.11 & 0.85 & 0.25 & 0.64 & 0.09 \\
\hline
\end{tabular}

There were no differences in mRNA expression after 1 day of zolpidem or diazepam. mRNA levels expressed as proportion of VEH controls. Modified with permission. Wright, B.T., C.F. Gluszek, and S.A. Heldt, The effects of repeated zolpidem treatment on tolerance, withdrawal-like symptoms, and GABAA receptor $m R N A$ s profile expression in mice: comparison with diazepam. Psychopharmacology (Berl), 2014. 231(15): p. 296779. 


\section{VITA}

Brittany T. Wright was born in Spartanburg, South Carolina, in 1988. She attended Clemson University where she earned a Bachelor's of Science in Psychology on May 2010 and will be receiving her Ph.D. in Biomedical Sciences with a concentration in Neuroscience from the University of Tennessee Health Science Center in August of 2016. 